\author{
UNIVERSIDAD POLITÉCNICA DE \\ VALENCIA
}

\title{
PROPUESTA DE MEJORA DE LOS GRÁFICOS DE CONTROL POR ATRIBUTOS MEDIANTE LA METODOLOGÍA DE DAUDIN
}

Tesis Doctoral

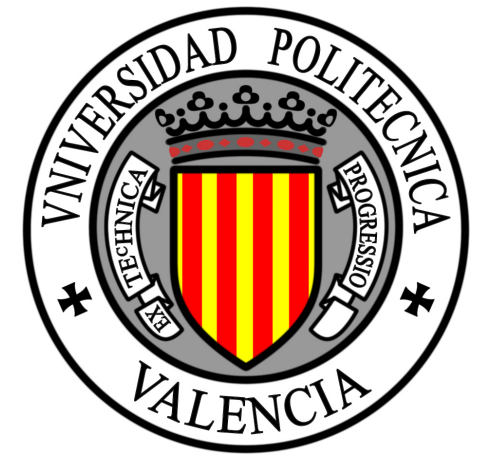

Por
Elena Pérez Bernabeu

2008 

DEPARTAMENTO DE ESTADÍSTICA E INVESTIGACIÓN OPERATIVA APLICADAS Y CALIDAD

UNIVERSIDAD POLITÉCNICA DE VALENCIA

\section{PROPUESTA DE MEJORA DE LOS GRÁFICOS DE CONTROL POR ATRIBUTOS MEDIANTE LA METODOLOGÍA DE DAUDIN}

Tesis Doctoral

Autor: Elena Pérez Bernabeu

Ingeniero de Organización Industrial

Directores: Dr. D. José Manuel Jabaloyes Vivas

Doctor Ingeniero Industrial

Dr. D. Andrés Carrión García

Doctor Ingeniero Industrial

2008 



\section{Tribunal:}

Presidente : Dr.D.Enrique Masiá Buades

Vocales : Dra.Dña. María Moreno Luzón

Dr.D. Mihir Sen

Dr.D. Eugenio Kahn Epprecht

Secretario : Dr.D. Gonzalo Clemente Marín

Suplentes : Dra.Dña. María Dolores Bovea Edo

Dr.D. Roberto Escuder Vallés

Acuerdan otorgar la calificación de:

Valencia, a

de de 2008 

A mi familia. 



\section{Índice general}

$\begin{array}{ll}\text { Agradecimientos } & \text { VI }\end{array}$

Resumen

Resum XII

$\begin{array}{ll}\text { Abstract } & \text { XVI }\end{array}$

1. Introducción y Objetivos 2

1.1. Antecedentes ..................... 2

1.2. Objetivos ....................... 10

2. Metodología 14

2.1. Secuencia de acciones . . . . . . . . . . . . . . . . . 14

2.2. Software empleado . . . . . . . . . . . . . . 17

3. Gráficos de Control para Atributos 20

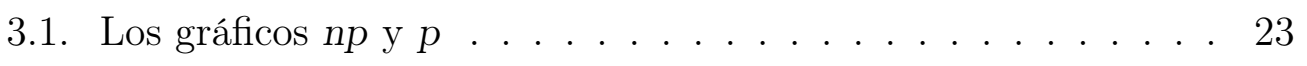

3.1.1. El gráfico $p \ldots \ldots$. . . . . . . . . . . . . . . . . . . . . . . . . . . . . . . . . . .

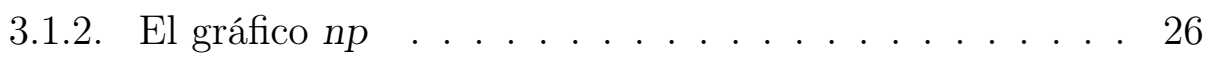

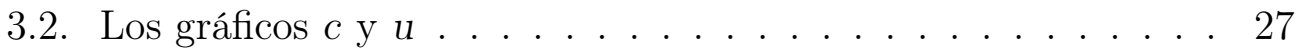

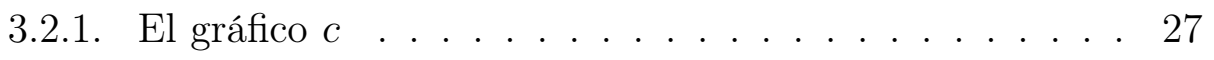

3.2.2. El gráfico $u$. . . . . . . . . . . . . . . . . . . 29

3.3. El muestreo doble . . . . . . . . . . . . . . . . . . . 31 
4. Algoritmos Genéticos 34

4.1. Origen. . . . . . . . . . . . . . . . . 34

4.1.1. Introducción . . . . . . . . . . . . . . 34

4.1.2. Descripción . . . . . . . . . . . . . . 38

4.2. Implementación . . . . . . . . . . . . . . . . . . . . . . 39

4.3. Software desarrollado . . . . . . . . . . . . . . . . . . 44

5. Propuesta de mejora del gráfico u: El gráfico DS-U 48

5.1. Metodología de Daudin . . . . . . . . . . . . . . . . . 48

5.2. Formulación del problema . . . . . . . . . . . . . 54

5.2.1. Gráfico $u$. . . . . . . . . . . . . . . 55

5.2.2. Gráfico $D S-U \ldots \ldots \ldots$. . . . . . . 56

6. Comparación de la potencia del gráfico $u$ frente a la potencia del gráfico $D S-U$ en un valor $u_{1} \quad 60$

6.1. Introducción. . . . . . . . . . . . . . . 60

6.2. Resultados. . . . . . . . . . . . . . . 63

6.2.1. Comparación de las curvas de potencia cuando el valor de diseño del gráfico $u$ clásico es $u_{0}=1 . \ldots$. . . . 63

6.2.2. Comparación de las curvas de potencia cuando el valor de diseño del gráfico $u$ clásico es $u_{0}=5$. . . . . . . 72

6.2.3. Comparación de las curvas de potencia en función del tamaño de muestra $n$, fijando $u_{0}$ y $\alpha \ldots \ldots$. . . . . 82

6.3. Conclusiones . . . . . . . . . . . . . . . . 85

6.4. Ejemplo . . . . . . . . . . . . . . . 86

7. Comparación de la potencia del gráfico $u$ frente a la potencia del gráfico $D S-U$ en $u_{1} \in\left[u_{\text {inferior }}, u_{\text {superior }}\right] \quad 90$

7.1. Introducción. . . . . . . . . . . . . . . . . 90

7.2. Metodología empleada. . . . . . . . . . . . . . . . . 91

7.3. Resultados . . . . . . . . . . . . . . . . . . 93 
7.3.1. Comparación de la potencia de los gráficos de control u y $D S-U$ con $n=8$ y $u_{0}=1 \ldots . . \ldots 93$

7.3.2. Comparación de la potencia de los gráficos de control u y $D S-U$ con $n=40$ y $u_{0}=1 \ldots$. . . . . . . 96

7.3.3. Comparación de la potencia de los gráficos de control u y $D S-U$ con $n=8$ y $u_{0}=5 \ldots 100$

7.4. Conclusiones . . . . . . . . . . . . . . . . . . . 104

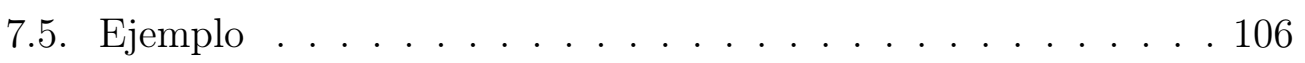

8. Conclusiones y Resultados 110

$\begin{array}{ll}\text { Bibliografía } & 114\end{array}$

$\begin{array}{ll}\text { Apéndices } & 121\end{array}$

A. Comparación de la potencia del gráfico $u$ frente a la potencia del gráfico $D S-U$ en un valor $u_{1}$

A.1. Comparación de los gráficos de control u y $D S-U$ con $n=8$, $u_{0}=1$ y $\alpha_{t}=0.01 \ldots \ldots \ldots$. . . . . . . . . . . 122

A.2. Optimización del gráfico de control $u$ con $n=8, u_{0}=1 \mathrm{y}$

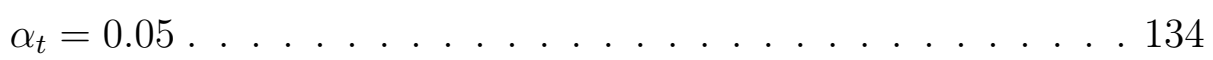

A.3. Comparación de los gráficos de control u y $D S-U$ con $n=8$, $u_{0}=1$ y $\alpha_{t}=0.0027 \ldots \ldots$. . . . . . . . . 146

A.4. Comparación de los gráficos de control u y $D S$ - $U$ con $n=40$,

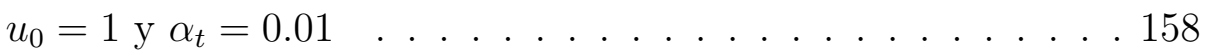

A.5. Comparación de los gráficos de control u y $D S$ - $U$ con $n=40$, $u_{0}=1$ y $\alpha_{t}=0.05 \ldots \ldots \ldots$. . . . . . . . . . 170

A.6. Comparación de los gráficos de control u y $D S-U$ con $n=40$,

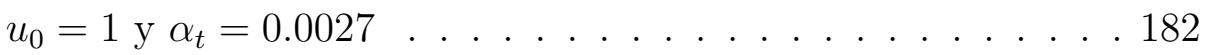

A.7. Comparación de los gráficos de control u y $D S-U$ con $n=8$, $u_{0}=5$ y $\alpha_{t}=0.01 \ldots \ldots \ldots \ldots$. . . . . . . . . . . . . . . . . 
A.8. Comparación de los gráficos de control u y $D S$ - $U$ con $n=8$, $u_{0}=5$ y $\alpha_{t}=0.05 \ldots \ldots \ldots \ldots$. . . . . . . . . . . . . . . . .

A.9. Comparación de los gráficos de control u y $D S-U$ con $n=8$, $u_{0}=5$ y $\alpha_{t}=0.0027 \ldots \ldots$. . . . . . . . . . 218

A.10.Comparación de los gráficos de control u y $D S-U$ con $n=40$,

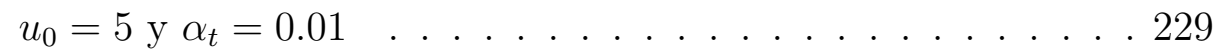

A.11.Comparación de los gráficos de control u y $D S-U$ con $n=40$,

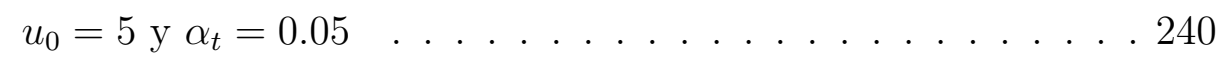

A.12.Comparación de los gráficos de control u y $D S-U$ con $n=40$, $u_{0}=5$ y $\alpha_{t}=0.0027 \ldots \ldots$. . . . . . . . . . 252

A.13.Comparación de los gráficos de control u y $D S-U$ en función del tamaño de muestra $n$, fijando $u_{0}$ y $\alpha \ldots \ldots 263$

B. Comparación de los gráficos de control u y $D S-U$ en $u_{0} \in$ $\left[u_{\text {inferior }}, u_{\text {superior }}\right]$

C. Código fuente para la optimización de la curva de potencia del gráfico $u$ en un valor $u_{1}$.

D. Código fuente para Optimización de la curva de potencia del gráfico $\boldsymbol{u}$ en $u_{0} \in\left[u_{i}, u_{s}\right]$ 


\section{Agradecimientos}

Deseo agradecer al Dr. José Jabaloyes Vivas y al Dr. Andrés Carrión García su dedicación y excelente dirección de esta tesis. Así mismo, le agradezco a la Dra. Ana Isabel Sánchez Galdón su gran ayuda en el encaminamiento de esta tesis al descubrirme la librería GAlib y ayudarme en la puesta en marcha del programa informático.

A mis padres, Ismael y Laura, por su comprensión y amor, agradeciéndoles de corazón su esfuerzo, apoyo y dedicación a lo largo de toda mi formación, que me ha permitido llegar hasta este momento.

Tampoco puedo dejar de agradecer a mi marido, Miguel Ángel, su infinita paciencia durante la realización de esta tesis, así como sus sabios consejos.

Por último, pero no menos importante, a mi hijo Miquel, que en sus cuatro años de vida nos ha dado muchas alegrías, que han permitido llevar mejor el esfuerzo de realización de esta Tesis Doctoral. Y también a la nueva alegría para la familia, Laura, que está de camino en el momento de la defensa de esta Tesis Doctoral. 



\section{Resumen}

TÍTULO : PROPUESTA DE MEJORA DE LOS GRÁFICOS DE CONTROL POR ATRIBUTOS MEDIANTE LA METODOLOGÍA DE DAUDIN

PRESENTADA POR : Elena Pérez Bernabeu

DIRIGIDA POR: José Manuel Jabaloyes Vivas y Andrés Carrión García

Los gráficos de control se pueden definir como una herramienta estadística que se utiliza para controlar un proceso. Permite al responsable del mismo distinguir entre las causas de variación que aparecen en el mismo. La variabilidad debida a causas comunes que nos permiten realizar predicciones sobre el estado del proceso o la variabilidad debida a causas especiales, que no nos permiten predecir la situación del proceso en un determinado momento.

Los gráficos de control se pueden clasificar, según la característica que se desee controlar, en dos grandes grupos: Gráficos de Control por Variables y Gráficos de Control por Atributos.

En la bibliografía existente se constata que hay numerosos estudios respecto a los gráficos por variables, en concreto, los gráficos para la media. En cambio hay menos aportaciones para los gráficos por atributos, que se están ampliando con el interés de muchos investigadores en los últimos años. 
Esta Tesis Doctoral se centra en la propuesta de mejora del gráfico $u$. Se aplica a este gráfico el muestreo doble propuesto por Daudin para el gráfico de la media (Daudin 1992). Se trata de un caso particular en dos etapas del muestreo múltiple.

El objetivo de esta Tesis Doctoral es conocer cómo se comporta la metodología de Daudin aplicada al gráfico $u$, y si mejora la potencia que ofrece el gráfico $u$ clásico.

La estructura de esta Tesis Doctoral consiste en ocho capítulos y un apartado de anexos:

Capítulo 1 : Presentación de los antecedentes, una breve introducción a los gráficos por atributos, así como los objetivos y la estructura de esta Tesis Doctoral.

CApítulo 2 : Se propone una metodología para la realización de esta Tesis Doctoral, y explican las técnicas y el software empleado.

Capítulo 3 : Define los gráficos por atributos, explicando detenidamente cada uno de ellos, en especial el gráfico $u$, sobre el cual se han aplicado modificaciones en esta Tesis Doctoral. También se hace un repaso de la bibliografía existente al respecto.

CApítulo 4 : Aborda una explicación detallada de la metodología de partida descrita por (Daudin 1992) y se muestra la formulación del problema para el gráfico $u$.

Capítulo 5 : Trata de la definición y explicación del funcionamiento de un Algoritmo Genético. Se ha utilizado para calcular los parámetros del gráfico $D S-U$ que ofrecen una mejora de la potencia del mismo frente a la potencia del gráfico $u$. 
Capítulo 6 : Se compara la potencia del gráfico $D S-U$ en un valor $u_{0}$ frente a la potencia del gráfico clásico $u$ en el mismo valor.

Capítulo 7 : Se analiza el comportamiento de la potencia cuando se pretende maximizar la potencia del gráfico $D S-U$ en un intervalo de valores $\left[u_{\text {inferior }}, u_{\text {superior }}\right]$ al mismo tiempo, en este caso, los que quedan más próximos al valor de diseño $u_{0}$, frente a la potencia del gráfico $u$ en ese mismo intervalo.

Capítulo 8 : Resume las conclusiones obtenidas en esta Tesis Doctoral, así como futuras líneas de investigación.

Anexos : Se muestran la totalidad de los datos y gráficos obtenidos en la simulaciones de los capítulos 6 y 7 . También se muestra la parte del código fuente del software empleado para ambos casos. 



\section{Resum}

TÍTOL : PROPOSTA DE MILLORA DELS GRÀFICS DE CONTROL PER ATRIBUTS EMPRANT LA METODOLOGÍA DE DAUDIN

PRESENTADA PER : Elena Pérez Bernabeu

DIRIGIDA PER: José Manuel Jabaloyes Vivas i Andrés Carrión García

Els gràifics de control es poden definir com una ferramenta estadística que s'utilitza per a controlar un procés. Permet al responsable del mateix distingir entre les causes de variació que apareixen en el procés. La variabilitat deguda a causes comunes que ens permeten realitzar prediccions sobre l'estat del procés o la variabilitat deguda a causes especials, que no ens permeten fer una predicció de la situació del procés en un determinat moment.

Els gràfics de control es poden classificar, segons la característica que es dessitge controlar, en dos grans grups: Gràfics de Control per Variables i Gràfics de Control per Atributs.

En la bibliografía existent es constata que hi ha nombrosos estudis respecte als gràfics per variables, en concret, els gràfics per a la mitja. En canvi hi ha menys aportacions per als gràfics per atributs, que s'estan ampliant amb l'interés de molts investigadors en els últims anys. 
Esta Tesi Doctoral se centra en la proposta de milora del gràfic u. S'aplica a este gràfic el mostreig doble proposat per Daudin per al gràfic de la mitja (Daudin 1992). Es tracta d'un cas particular en dos etapes del mostreig múltiple.

L'objetiu d'esta Tesi Doctoral és conèixer com es comporta la metodología de Daudin aplicada al gràfic $u$, i si millora la potència que ofereix el gràfic u clàsic.

L'estructura d'esta Tesi Doctoral consisteix en huit capítols i un apartat d'annexes:

CAPÍtol 1 : Presentació dels antecedents, una breu introducció als gráfics per atributs, així com els objectius i l'estructura d'esta Tesi Doctoral.

Capítol 2 : Es proposa una metodología per a la realització d'esta Tesi Doctoral, i s'expliquen les tècniques i el software emprat.

Capítol 3 : Es defineixan els gràfics per atributs, explicant detingudament cadascun d'ells, en especial, el gràfico $u$, al qual s'han aplicat modificacions en esta Tesi Doctoral. També es fa un repàs de la bibliografía existent al respecte.

CApítol 4 : Aborda una explicació detallada de la metodología de partida descrita per (Daudin 1992) i es mostra la formulació del problema per al gràfic $u$.

Capítol 5 : Tracta de la definició i explicació del funcionament d'un Algorisme Genètic. S'ha emprat per a calcular els paràmetres del gràfico $D S-U$ que ofereixen una millora de la potència del mateix front a la potència del gràfic $u$.

Capítol 6 : Es compara la potència del gràfic $D S-U$ en un valor $u_{0}$ front a la potència del gràfic clàssic $u$ en el mateix valor. 
CApítol 7 : S'analitza el comportament de la potència quan es pretém maximitzar la potència del gràfic $D S-U$ en un interval de valors $\left[u_{\text {inf. }}, u_{\text {sup. }}\right]$ al mateix temps, en este cas, els que queden més pròxims al valor de disseny $u_{0}$, front a la potència del gràfic $u$ en eixe mateix interval.

Capítol 8 : Resumeix les conclussions obtingudes en esta Tesi Doctoral, així com futures línies d'investigació.

AnNeXes : Es mostren la totalitat de les dades i gràfiques obtingudes en les simulacions dels capítols 6 i 7. També es mostra la part del codi font del software emprat per a ambdós casos. 



\section{Abstract}

TITLE : PROPOSAL FOR THE IMPROVEMENT OF ATTRIBUTE CONTROL CHARTS USING DAUDIN'S METHODOLOGY

PRESENTED BY : Elena Pérez Bernabeu

DIRECTED BY : J. Manuel Jabaloyes Vivas and Andrés Carrión García

Control charts can be defined as a statistical tool used for controlling processes. It allows to the process responsible to distinguish between the reasons of variation that can appear: The variability owed to common reasons that allow us to predict the state of the process or the variability owed to special reasons, which do not allow us to predict the situation of the process in a certain moment.

We can classify Control charts, according to the characteristic to be controlled, in two big groups: Variable Control charts and Attribute Control charts.

It is stated that in the existing bibliography there are several studies about variable control charts, concretely, average control charts. On the other hand there are fewer contributions for the attribute control chart, which are beginning to be developed by some researchers in the last years. 
This Doctoral Thesis is centered in the proposal of the $u$ control chart improvement. It is applied to this chart the Double Sampling designed by Daudin for the average chart (Daudin 1992). It is a particular case of the multiple sampling.

The main aim of this Doctoral Thesis is to know how Daudin's methodology is performed on the $u$ control chart, and if in this cas, the power obtained is better than the one in the classic $u$ chart.

This Doctoral Thesis structure consist of eight chapters and a part of annexes:

Chapter 1 : The precedents are introduced, a brief introduction to the attribute charts, as well as the aims and the structure of this Doctoral Thesis.

Chapter 2 : A proposal of methodology is given for this Doctoral Thesis, and it is also explained the technologies and the specific software.

Chapter 3 : It is defined the attribute charts, explaining with detail each of them, especially the $u$ graph, on which modifications have been applied in this Doctoral Thesis. Also there is done a revision of the existing bibliography in this topic.

Chapter 4 : It is detailed an explanation of the methodology described in (Daudin 1992) and it is shown the formulation of the problem for the $u$ graph.

Chapter 5 : It is defined and explained the performance of a Genetic Algorithm. It has been used for calculating the $D S-U$ chart parameters that improve its power comparing to the $u$ chart power.

Chapter 6 : The $D S-U$ chart power in a value $u_{0}$ is compared with the power of the classic $u$ chart in the same value. 
Chapter 7 : The behavior of the power is analyzed when it is tried to maximize the power of the $D S-U$ chart in an interval of values $\left[u_{\text {Inferior }}, u_{\text {Superior }}\right]$ at the same time, in this case, those values which remain next to the value of design $u_{0}$, opposite to the power of the $u$ chart in the same interval.

Chapter 8 : The conclusions obtained are summarized in this Doctoral Thesis, as well as the future research lines.

AnNexes : It is shown all the information and charts referred to in chapters 6 and 7 . It is also shown partially the software source code used. 



\section{Capítulo 1}

\section{Introducción y Objetivos}

\subsection{Antecedentes}

La Calidad se ha convertido en los últimos años en uno de los factores decisivos más importante entre los consumidores a la hora de elegir un producto o servicio.

La historia del concepto de la calidad se remonta a la preocupación por el trabajo bien hecho, por tanto, siempre ha existido un concepto intuitivo de la calidad.

La Revolución Industrial impulsó el campo de la calidad, pero sobre todo, el desarrollo de herramientas estadísticas y gerenciales que ocurre durante el siglo XX. El consumidor, tanto institucional como el particular, más exigente cada día, y la fuerte competencia nacional e internacional, provocan una evolución constante en las bases filosóficas y en la práctica de la Gestión de la Calidad. 
La II guerra mundial fue el catalizador que permitió ampliar el cuadro de control a diversas industrias en los Estados Unidos, cuando la simple reorganización de los sistemas productivos resultó inadecuada para cumplir las exigencias del estado de guerra y semiguerra.

Los gráficos de control se pueden definir como una herramienta estadística que se utiliza para controlar un proceso. Permite al responsable del mismo distinguir entre las causas de variación que aparecen en el mismo:

- Variabilidad debida a causas comunes, que nos permite realizar predicciones sobre el estado del proceso.

- Variabilidad debida a causas especiales, que no nos permiten predecir la situación del proceso en un determinado momento.

Un gráfico de control nos facilita (Carot 1998):

1. Vigilancia y control del proceso evitando la producción de defectos. Sigue la filosofía: hágalo bien a la primera.

2. Aumento de la homogeneidad de la producción mediante la disminución de la variabilidad del proceso.

3. Evita los ajustes innecesarios, con el lema de si no está roto, no lo repares.

4. Proporciona información sobre los parámetros del producto y del proceso, lo que permitirá conocer mejor nuestra actividad productiva.

Los gráficos de control se pueden clasificar en dos grandes grupos en función de la característica de calidad que se controle: 
Gráficos de Control por Variables: La característica de calidad es una variable, que puede medirse y expresarse como un número en una escala de medición continua.

Gráficos de Control por Atributos: Muchas características de calidad no se miden en una escala continua o en una escala cuantitativa. En estos casos, cada unidad del producto puede juzgarse como conforme o disconforme en base a si posee o no ciertos atributos, o puede contarse el número de disconformidades (defectos) que aparecen en una unidad del producto.

Existen gráficos de control para la media, la dispersión, la proporción de piezas defectuosas y la proporción de defectos o sus frecuencias. Para controlar la media destacamos los siguientes, entre otros muchos:

- Media Muestral (Shewhart 1931)

- Medias Móviles (Montgomery 1991)

- CUSUM (Page 1954; Lucas and Saccucci 1990)

- EWMA (Roberts 1959; Crowder 1987)

Y además, se puede aplicar cada uno de estos gráficos con distintas metodologías:

- Parámetros constantes (PC)

- VSS (Variable Sample Size). No se mantiene el tamaño de muestra constante, sino que que se adapta al proceso, en función de su comportamiento. (Prahbu, Runger, and Keats 1993; Costa 1994)

- VSI (Variable Sample Interval). No se mantiene el intervalo de muestreo constante, sino que se varía en función del comportamiento del proceso. (Reynolds Jr et al. 1988)

- VSSI (Variable Sample Size and Sampling Interval). Es una combinación de VSS y VSI. (Costa 1997) 
- Metodología de Daudin (Daudin 1992)

Para el control de la dispersión, los gráficos más habituales son: Rango Muestral, Desviación Típica, Cuasidesviación Típica y Cuasivarianza, con sus correspondientes CUSUM, y EWMA, y las metodologías utilizadas por estos gráficos, como la para la media: Parámetros Constantes y Parámetros Variables (VSS, VSI y VSSI).(Montgomery 1991)

Por último, hay cuatro tipos de gráficos de control para atributos según la naturaleza del control a realizar, que son los siguientes:

- Basados en la distribución Binomial:

Gráfico np- Número de unidades defectuosas en muestras de tamaño constante.

Gráfico $p$ - Porcentaje de unidades defectuosas en muestras de tamaño variable.

- Basados en la distribución de Poisson:

Gráfico c - Número de defectos por muestra en muestras de tamaño constante.

Gráfico $u$ - Número de defectos por unidad en muestras de tamaño variable.

Existe una amplia bibliografía referente a los gráficos por variables, pero en cambio hay menos aportaciones para los gráficos por atributos. En los últimos años se ha aumentado los estudios relativos a los gráficos por atributos, entre los cuales destacamos cronológicamente algunos como:

(Chan, Xie, and Goh 2000): Los autores proponen un nuevo tipo de gráfico de control denominado gráfico de control de cantidades acumuladas (gráfico CQC), para poder corregir el comportamiento insatisfactorio de los gráficos de control c y $u$, ya que éstos no son adecuados 
para controlar procesos de alto rendimiento con tasas de defecto bajas. Este nuevo gráfico propuesto se puede emplear sin importar que la tasa de defectos sea alta o baja, y cuando la tasa de defectos del proceso es baja o moderada, el gráfico CQC no tiene la deficiencia de los gráficos c y u de mostrar señales de falsa alarma demasiado frecuentemente. El gráfico $\mathrm{CQC}$ no requiere subgrupos racionales de muestras (que sí se necesita para los gráficos c y u), y es apropiado para controlar procesos de fabricación automática.

(Epprecht and Costa 2001): Se debate sobre el uso del gráfico np con tamaño de muestra variable para controlar procesos de alto rendimiento que requieren una acción rápida frente a saltos moderados o incluso pequeños en el número de unidades defectuosas lanzadas durante la producción. En otros casos donde el proceso no requiere acción rápida contra este tipo de alteraciones, se puede utilizar la característica VSS, al menos, para reducir el número de elementos que se toman del proceso para ser analizados. También se trasladaron estos resultados al gráfico $c$ con VSS.

(Hamada 2002): La probabilidad contenida en los límites de control estándar para atributos puede variar porque los parámetros de la distribución que aparecen en los límites de control están estimados en base a información anterior. Este artículo propone utilizar límites de control por intervalos de tolerancia Bayesiana que controla la probabilidad contenida en un nivel especificado con una confianza determinada. Se desarrollan estos límites y se muestran ejemplos para los cuatro tipos de gráficos por atributos: $n p, p, c$ y $u$.

(Luo and Wu 2002): En este artículo se desarrolla un algoritmo para los diseños optimizados del gráfico de control np adaptativo para controlar la fracción de unidades defectuosas de un proceso, $p$. Se incluye VSS y VSI, demostrando que mejoran la efectividad significativamente, espe- 
cialmente para detectar cambios pequeños o moderados en el proceso. Los algoritmos para la optimización de problemas se implementaron en lenguaje $C$ y que se puede solicitar a los autores. Además se muestran diagramas de flujo para el diseño óptimo de cada uno de los gráficos de control adaptativos.

(Fang 2003): En este artículo, el autor analiza el caso en el que, teniendo un gráfico $c$, no se puede asumir que la ocurrencia de defectos en muestras está bien modelada por una distribución de Poisson. En ese caso se suele emplear el gráfico $X$. El autor compara los méritos de los gráficos c y $X$ para la familia de distribuciones Katz con respecto a la distribución de Poisson.

(Wang and Ma 2003): En esta investigación se presenta un esquema optimizado de diseño de gráficos de control por atributos np-VSS ( Variable Sample Size), demostrando que su comportamiento es significativamente mejor que los gráficos tradicionales debido a que los parámetros de diseño son adaptativos y obtenidos a través de procedimientos de optimización cuyo objetivo es conseguir propiedades de ARL (Average Run Length) óptimas.

(Wu and Luo 2004): Como en recientes estudios se demuestra que los gráficos de control adaptativos son más rápidos que los gráficos estáticos clásicos en la detección de cambios en el proceso, aquí se desarrolla un algoritmo para los diseños optimizados del gráfico de control $n p$ adaptativo para controlar la fracción de unidades defectuosas de un proceso, $p$. Se incluye VSS, VSI y VSSI, demostrando que mejoran la efectividad significativamente, especialmente para detectar cambios pequeños o moderados en el proceso. 
(Khoo 2004): Este autor analiza el gráfico $c$ utilizado para monitorizar el número de defectos en una unidad de inspección de producto, especialmente cuando la probabilidad de ocurrencia de un defecto es relativamente pequeña. Sin embargo, el gráfico $c$ es lento en la detección de cambios pequeños. A cambio propone una alternativa más eficiente basada en la construcción de un gráfico de control de medias móviles de Poisson para el número de defectos. Se hace una demostración de los ARL (Average Run Length) de ambos gráficos, mostrando que la nueva aproximación ofrece una detección más rápida de las condiciones de fuera de control, mientras que a la vez consigue un ARL-bajo control más largo.

(Chakraborti and Human 2006): En este artículo se analiza la estimación de parámetros y el comportamiento del gráfico $p$, centrándose sobretodo, en las tasas de falsa alarma $(\alpha)$ y en el ARL bajo control. Se demuestra que cuando se ha de estimar el valor de $p$, entonces las recomendaciones habituales de 10 a 20 subgrupos de tamaño 5 son insuficientes para obtener un $\alpha=0,0027$. Se ofrecen las fórmulas y reglas para alcanzar los objetivos de $\alpha$ y $A R L$ que se fijen, siendo que el gráfico $p$ es una distribución discreta (binomial), en la que es bastante improbable ser capaz de garantizar una tasa de falsa alarma - $\alpha$ - exacta como es habitual en gráficos de control por variables.

(Lucas, Davis, and Saniga 2006): En este estudio se presenta un método para mejorar la información proporcionada por los gráficos c y np. Se trata de aquellos casos en particular en los que el límite de control inferior es cero, y se busca señalizar un incremento de la calidad del procesos cuando se contabiliza en $k$ o más muestras que ocurren 0 defectos por unidad de inspección ó 0 unidades defectuosas. 
(Wu, Luo, and Zhang 2006): En este artículo se presenta un algoritmo para la optimización del diseño del gráfico $n p$, proponiendo un diseño reducido (curtailment) para maximizar la efectividad del gráfico $n p$ manteniendo la tasa de falsa alarma a un nivel específico y su sencillez de comprensión y operación. Este algoritmo se puede aplicar a una inspección $100 \%$ así como a inspecciones generales por muestreo aleatorio. Como resultado se ofrece la reducción hasta la mitad del ATS fuera de control (Average Time to Signal) comparado con los gráficos np convencionales.

(Kaya and Engin 2007): En este artículo proponen una solución para definir el tamaño de muestra en gráficos de control por atributos desarrollando procesos en varias fases y resolviéndolo mediante algoritmos genéticos. Se detalla un caso aplicado al proceso de producción de pistones, para el que se define un plan de inspección muestral: dado un tamaño de lote se obtiene $n$ y un número de aceptación $c$ (número de unidades defectuosas máximas que se han de encontrar en el lote para considerar el lote como bueno).

Pero todavía hay un gran vacío en la investigación de los gráficos de control por atributos si comparamos con los estudios para los gráficos para la media. Además, la mayoría de los estudios que se vienen realizando se centran, sobre todo, en el diseño de gráficos de control adaptativos ${ }^{1}$, y muy pocos sobre el muestreo múltiple ${ }^{2}$, en dos o más etapas. En el apartado 3.3 se puede ver la diferencia entre los artículos encontrados en este campo para

\footnotetext{
${ }^{1}$ Los parámetros que caracterizan este tipo de gráficos de control no se mantienen constantes, sino que se ajustan a los resultados que se van obteniendo del proceso, por ejemplo, cuando hay una evidencia de que el proceso está fuera de control, se puede fijar una norma que acorte el tiempo hasta la siguiente muestra

${ }^{2} \mathrm{El}$ muestreo múltiple consiste en la toma de decisiones a partir de dos o más etapas de muestreo, frente a una única etapa habitual en los gráficos clásicos
} 
gráficos de control por variables, y los encontrados para gráficos de control por atributos. Este trabajo se va a centrar en el gráfico de control $u$ de defectos, proponiendo un gráfico nuevo, que consiste en aplicar la metodología de Daudin (Daudin 1992) al gráfico $u$.

\subsection{Objetivos}

Con esta Tesis Doctoral se pretende mejorar la potencia de los gráficos por defectos, concretamente, del gráfico $u$. La potencia es la probabilidad de que un gráfico de control detecte las salidas de control cuando el proceso está fuera de control. Para ello se define el gráfico $D S-U^{3}$, y se estudia la mejora de la potencia de éste frente al gráfico $u$ original.

Así pues, los objetivos de esta Tesis Doctoral son:

Objetivo General

- Definir un gráfico de control DS-U que permita mejoras en la potencia del gráfico $u$ clásico.

OBjetivos Específicos

- Estudiar los fundamentos estadísticos del control de procesos por atributos.

- Estudiar el uso de algoritmos genéticos como herramientas de análisis y mejora de los gráficos de control estudiados.

- Identificar los parámetros de diseño del gráfico DS-U

\footnotetext{
${ }^{3}$ Las siglas DS-U se refieren al gráfico de control que se propone en esta Tesis Doctoral, aplicando Doble Muestreo, al gráfico de control $u$. Se explica en el capítulo 5.
} 
- Establecer los criterios para evaluar el gráfico $D S-U$ frente al gráfico $u$.

- Definir estrategias de mejora en dos diferentes situaciones:

- En un valor $u_{1}$ (Capítulo 6),

- En un intervalo de valores $u_{1} \in\left[u_{\text {inferior }}, u_{\text {superior }}\right]$ (Capítulo 7).

La presente Tesis Doctoral se ha estructurado en ocho capítulos que se describen a continuación:

El CAPÍTULO 1 presenta los antecedentes, una breve introducción a los gráficos por atributos, así como los objetivos y la estructura de esta Tesis.

En el CAPÍTULO 2 se propone una metodología para la realización de esta Tesis Doctoral, y explican las técnicas y el software empleado.

El CAPÍTUlo 3 se centra en la definición de los gráficos por atributos, explicando detenidamente cada uno de ellos, en especial el gráfico $u$, sobre el cual se han aplicado modificaciones en esta Tesis Doctoral. También se hace un repaso de la bibliografía existente al respecto.

En el CAPÍtulo 4 se define y se explica el funcionamiento de un Algoritmo Genético. Esta es la herramienta que se ha elegido para estudiar las posibles mejoras resultantes de aplicar la metodología de Daudin al gráfico $u$.

La aportaciones de esta Tesis Doctoral se detallan en los siguientes capítulos:

En el CAPÍTULO 5 se hace una explicación detallada de la metodología de partida descrita por (Daudin 1992) y se muestra la formulación del problema para el gráfico $u$. 
El CAPÍTULO 6 estudia el comportamiento de la potencia cuando se quiere maximizar el valor de la potencia en un valor $u_{1}$ en el gráfico $D S-U$ frente al valor de la potencia en el gráfico $u$, determinando los parámetros óptimos para el diseño del gráfico $D S-U$ propuesto. Se analiza detalladamente las soluciones obtenidas para distintas combinaciones de parámetros de un gráfico clásico $u$, es decir, tamaño de muestra, error de primera especie y $u_{0}$ (número de defectos por muestra) fijados.

El CAPÍTULO 7 analiza la tendencia de la potencia cuando se pretende maximizar la potencia del gráfico $D S-U$ en un intervalo de valores [ $u_{\text {inferior }}, u_{\text {superior }}$ ] al mismo tiempo, en este caso, los que quedan más próximos al valor de diseño $u_{0}$, frente a la potencia del gráfico $U$ en ese mismo intervalo.

Por último, el CAPÍTULO 8 versa sobre las conclusiones obtenidas en esta Tesis Doctoral, así como futuras líneas de investigación.

Tras los capítulos se recogen una serie de ANEXOs en los que se muestran la totalidad de los datos y gráficos obtenidos en la simulaciones de los capítulos 6 y 7. También se muestra la parte del código fuente empleado en el software diseñado, y que complementa a la librería GALib para la obtención de resultados. 



\section{Capítulo 2}

\section{Metodología}

\subsection{Secuencia de acciones}

Para la realización de esta Tesis Doctoral se han seguido los siguientes pasos:

1. Planteamiento del objeto de la Tesis Doctoral.

En la revisión de la bibliografía se detecta una carencia en el estudio de los gráficos por atributos, por lo que se toma la decisión de trabajar en este sentido. Al ver el trabajo realizado para los gráficos de control para la media por parte de J.J.Daudin (Daudin 1992), se propone aplicarlo al gráfico por atributos $u$.

2. Documentación.

Se inicia una fase de documentación para tener un mayor conocimiento del estado de la investigación en el tema de gráficos de control en general, y de gráficos por atributos en particular. Se busca información en la 
Biblioteca de la Universidad Politécnica de Valencia - tanto in situ, con revistas en formato papel, como mediante el Polibuscador o Biblioteca Virtual de la UPV-, y también a nivel de internet, con buscadores como Google. En los capítulos 3 y 5 se ha reflejado la información extraída de dicha búsqueda.

3. Formulación del problema.

Formulación estadística del nuevo gráfico de control propuesto, denominándolo DS-U (Double Sampling U).

En el capítulo 3 se muestra la formulación del gráfico por atributo clásico, y la formulación para el gráfico por atributos $D S-U$ planteado en esta Tesis Doctoral.

Con esta formulación estadística del problema se buscan los parámetros óptimos que caractericen este nuevo gráfico de control $D S-U$ que tratarán de mejorar las prestaciones del gráfico clásico $u$.

4. Selección de la técnica a emplear.

Se opta por aplicar la técnica de algoritmos genéticos, como se hace referencia en algunos artículos de la bibliografía (Martorell et al. 2000), (He, Grigoryan, and Sigh 2002), etc.

5. Programación de una aplicación informática.

Este programa debe recoger la información tratada en los pasos 1, 2, 3 y 4. Se elige programar dicha aplicación en lenguaje $\mathrm{C}++\mathrm{y}$ también se decide utilizar una librería de uso libre, GAlib. En los anexos C y D se puede ver el código fuente de dichos programas.

6. Realización de ensayos con el programa

Para ello se ha hecho uso del laboratorio 3L4 del aulario Ferrándiz de la Escuela Politécnica Superior de Alcoy (E.P.S.A.) para la realización del trabajo experimental. 


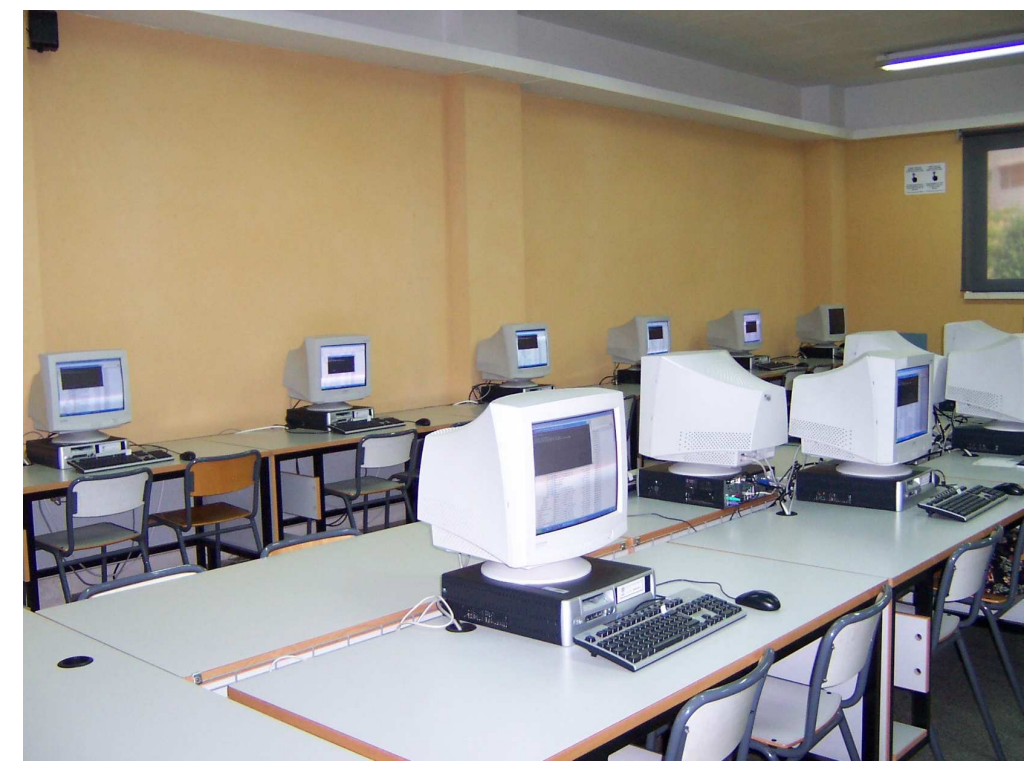

Fig. 2.1: Laboratorio de realización de simulaciones

Se trata de un aula de informática, con 24 ordenadores, con las siguientes características:

- Pentium ${ }^{\circledR} 4$ a 2’80 GHz

- 512 MB RAM

- 15GB HDD

7. Análisis de datos A partir de las soluciones obtenidas por el programa (apartado 5), se realiza un análisis de los datos, y del funcionamiento de la nueva metodología en el gráfico de control $u$.

8. Obtención de conclusiones

Una vez analizados los datos, se pasa a obtener conclusiones que detallen la mejora obtenida por el planteamiento inicial propuesto en el apartado 1 . 


\subsection{Software empleado}

En cuanto a los programas informáticos empleados para la realización de esta Tesis Doctoral, los podemos dividir en dos grupos, según para qué han sido utilizados:

- Para realizar la aplicación informática que obtenDRÁ LAS SOLUCIONES ÓPTIMAS.

\section{- Borland $\mathrm{C}++$.}

Para la realización de la aplicación informática que permitirá al responsable de calidad obtener los valores para el diseño del gráfico $D S-U$ se programado la misma en lenguaje $\mathrm{C}++$, integrando la librería GAlib en la misma.

\section{- Borland $\mathrm{C}++$ Builder.}

La interfaz gráfica se ha realizada con esta aplicación informática, para así obtener un entorno de Windows amigable para el usuario. Además, permite una fácil modificación de los parámetros de partida del algoritmo genético que se ejecutará. Se puede ver la pantalla de toma de datos en la figura 2.2.

\section{- Librería GAlib.}

GAlib es una librería de $\mathrm{C}++$ con objetos de algoritmos genéticos. La librería incluye herramientas para utilizar algoritmos genéticos cuyo objetivo sea optimizar en cualquier programa de $\mathrm{C}++$ utilizando cualquier representación y distintos operadores genéticos. El código fuente de GAlib no es de dominio público, aunque está disponible sin coste para propósitos sin ánimo de lucro. Matthew Wall, el autor de GAlib empezó este proyecto como un gran ejercicio de programación tras haber utilizado una librería de algoritmos 


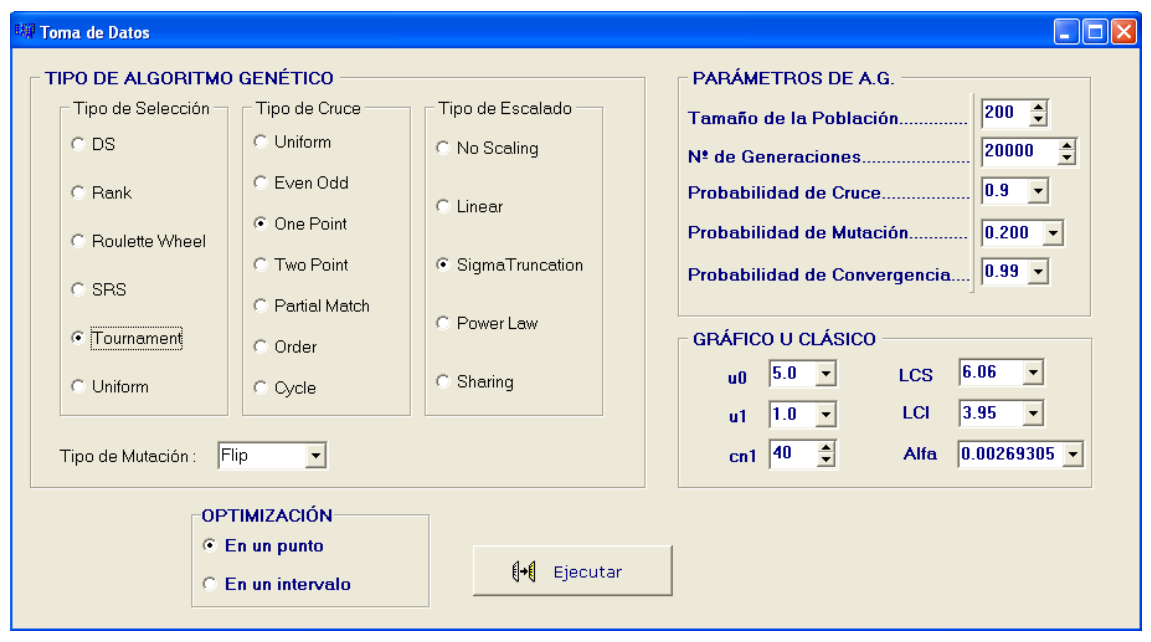

Fig. 2.2: Pantalla de toma de datos

genéticos escrita por Kazu Saito en el MIT CADlab para su tesis doctoral. Fue ampliando el programa con más funciones cada vez que necesitaba desarrollar alguna nueva hasta que GAlib hizo su primera aparición pública en la primavera de 1995. GAlib fue diseñado para hacer fácil la elección de diferentes funciones objetivo, representaciones, operadores genéticos, y algoritmos genéticos. Incluye diferentes representaciones, operadores genéticos, algoritmos genéticos, criterios de parada, métodos de escalado, métodos de selección y esquemas de evaluación.

Todo GAlib (fuente y documentación) está protegido por la Convención de Berna. Cualquier publicación de trabajos basados en experimentos que utilicen GAlib deben incluir un reconocimiento adecuado de GAlib.

Cuando utilizamos la librería, se trabaja básicamente con dos clases: el genoma y un algoritmo genético. Cada instancia de un genoma representa una solución individual de nuestro problema, el objeto algoritmo genético define cómo debe tener lugar la evo- 
lución. Este algoritmo genético utiliza una función objetivo que se ha de definir en cada caso particular para determinar cómo se ajusta cada genoma para su supervivencia. Utiliza los operadores de genoma (construidos dentro del genoma) y las estrategias de selección y reemplazamiento (construidas dentro del algoritmo genético) para generar nuevos individuos. Con esta librería tan sólo se ha de programar la función objetivo que determinará qué individuos han de sobrevivir, cuáles han de reproducirse y cuáles han de morir.

En la sección 4.2 se dan más detalles de la utilización de esta librería.

- Para analizar las soluciones obtenidas CON el aNteRIOR.

Se ha empleado el programa Mathcad ${ }^{\circledR} 2000$ de MathSoft, Inc. Ha servido para hacer los cálculos relativos a los gráficos de control u y $D S-U$, así como para la representación gráfica de la potencia $(1-\beta)$ en las curvas de potencia, el tamaño medio de muestra y del diferencia entre ambas potencias. 


\section{Capítulo 3}

\section{Gráficos de Control para Atributos}

Los gráficos de control por atributos constituyen la herramienta esencial utilizada para controlar características de calidad con sólo dos situaciones posibles, como por ejemplo: conforme/disconforme, funciona/ no funciona, defectuoso/no defectuoso, presente/ausente, etc.; o bien para características que se puedan contar, como número de manchas, número de golpes, número de rayas, etc. También en algunas ocasiones se tratan características por variables como atributos, en el caso de que sólo se considere si se cumplen o no las especificaciones ${ }^{1}$ de calidad sin importar cuál es el valor concreto de dicha variable.

Por lo general, los gráficos por atributos no ofrecen tanta información como los gráficos por variables, ya que una medición numérica es más informativa que la sola clasificación de una unidad como conforme o disconforme.

\footnotetext{
${ }^{1}$ Son las medidas deseadas de las características de la calidad en un producto. Las características de calidad se evalúan con respecto a estas especificaciones.
} 
Aún así, los gráficos por atributos son muy útiles en el sector servicios y en los esfuerzos de mejora de la calidad fuera de la manufactura, ya que no es fácil medir en una escala numérica un gran número de las características de calidad que se encuentran en estos escenarios.

Al igual que en los gráficos de control por variables, el gráfico de atributos representa un estadístico $T$ del proceso (como puede ser el número de defectos) frente al número de la muestra o al tiempo. Una línea central representa el valor medio o esperado del estadístico, mientras que la especificación de los límites de control es una de las decisiones críticas que deben tomarse al diseñar un gráfico de control.

Un punto que se encuentra fuera de los límites de control se interpreta como una evidencia de que el proceso está fuera de control.

Además, incluso si todos los puntos se hallan comprendidos entre los límites de control, pero se comportan de manera sistemática o no aleatoria, también se tendría un proceso fuera de control.

En el uso de este tipo de gráficos han de considerarse las siguientes limitaciones:

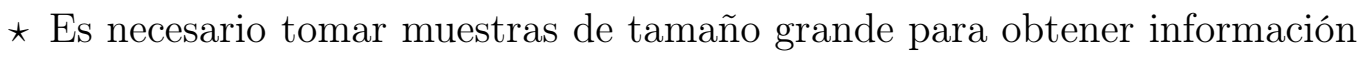
significativa.

* Son aplicables a procesos que presentan cantidades considerables de disconformidades (defectos), o unidades no conformes (defectuosas).

* No avisan de cambios adversos en el parámetro que queremos controlar en el proceso hasta que se han registrado un mayor número de defectos o unidades no conformes.

$\star$ Las verificaciones pueden estar influidas por subjetividades de las personas que evalúan la muestra, por lo que se hace necesario el establecimiento de unos criterios de conformidad escritos y con apoyo de medios visuales que 
minimicen estas diferencias.

Por otra parte, el uso de estos gráficos de control comporta las siguientes ventajas:

- La recogida de información de atributos es rápida y poco costosa.

- Se pueden aplicar a cualquier tipo de característica.

- Permiten identificar las causas especiales de variación que afectan al proceso cuando los valores representados en la gráfica se salen de los límites de control especificados, es decir, cuando el proceso está estadísticamente fuera de control.

Las aplicaciones más frecuentes de estos gráficos son las siguientes:

- En el control de características de calidad del tipo conforme/defectuoso o muy costosas de medir en una escala numérica.

- En una primera toma de información en el proceso para detectar las operaciones que provocan más defectos.

- En el control de procesos que generan cantidades grandes de defectos.

En este capítulo se presentan gráficos de control por atributos en dos grupos:

1. Se puede comparar un producto con un estándar y se clasifica como defectuoso o no (gráficos $p$ y np)

2. En algunos productos, la existencia de un defecto no necesariamente conlleva a que el producto sea defectuoso; en tales casos, puede resultar conveniente clasificar un producto según el número de defectos que presenta (gráficos c y u). 
Es importante notar que los gráficos $p$ y u permiten trabajar con muestras de tamaños diferentes, mientras que los gráficos np y c están diseñados para muestras de tamaño constante.

\subsection{Los gráficos $n p$ y $p$}

Los gráficos np y $p$ se utilizan para controlar la proporción de piezas defectuosas que genera el proceso; el primero exige que el tamaño de muestra sea constante mientras que el segundo no. (Montgomery 1991)

\subsubsection{El gráfico $p$}

El gráfico $p$ es un gráfico de control del porcentaje o fracción de unidades defectuosas (cociente entre el número de artículos defectuosos en una población y el número total de artículos de dicha población).

Este tipo de gráfico se basa en la evaluación del número de unidades defectuosas en muestras de tamaño variable tomadas a intervalos fijos de tiempo. Se utiliza cuando en un muestreo no puede mantenerse constante el tamaño de muestra.

Generalmente se requiere un tamaño de muestra grande, tanto para este gráfico de control como para el resto de gráficos de control por atributos. Este tamaño de muestra ha de ser lo suficiente como para que en cada muestra se registren varias unidades defectuosas, de forma que puedan evidenciarse cambios significativamente favorables, como la aparición de muestras con cero unidades defectuosas. 
Los principios estadísticos que sirven de base al diagrama de control $p$ se basan en la distribución Binomial: supóngase que el proceso de producción funciona de manera estable, de tal forma que la probabilidad de que cualquier artículo no esté conforme con las especificaciones es $p$, y que los artículos producidos sucesivamente son independientes. Representando por $X_{i}$ al número de artículos defectuosos en la muestra i-ésima, tendremos que $X_{i} \approx B\left(n_{i}, p\right)$.

- Sabemos que: $\mu_{X_{i}}=n_{i} p$ y $\sigma_{X_{i}}=\sqrt{n_{i} p(1-p)}$

- Para cada muestra, se define la variable aleatoria fracción disconforme muestral como: $\hat{p}_{i}=\frac{X_{i}}{n_{i}}$. Observese que $\hat{p}_{i}$ seguirá una distribución con media y desviación típica:

$$
\begin{gathered}
E\left[\hat{p}_{i}\right]=\frac{E\left[X_{i}\right]}{n_{i}}=p \\
\operatorname{Var}\left[\hat{p}_{i}\right]=\frac{\operatorname{Var}\left[X_{i}\right]}{n_{i}^{2}}=\frac{p(1-p)}{n_{i}}
\end{gathered}
$$

- Por tanto, si $n$ es suficientemente grande, se puede aplicar el Teorema Central del Límite y aproximadamente:

$$
\hat{p}_{i} \longrightarrow N\left(p, \sqrt{\frac{p(1-p)}{n_{i}}}\right) \text {, si } n_{i} \rightarrow \infty
$$

- Los límites de control y la media de este gráfico se calcularán del siguiente modo:

$$
\begin{gathered}
L C S=p+z_{\frac{\alpha}{2}} \sqrt{\frac{p(1-p)}{n_{i}}} \\
\text { Línea central }=p \\
L C I=p-z_{\frac{\alpha}{2}} \sqrt{\frac{p(1-p)}{n_{i}}}
\end{gathered}
$$

siendo $\alpha$ la probabilidad de detectar una salida de control cuando el proceso está bajo control. 
- Si $p$ es desconocida, se puede estimar (observar que tal estimación se realizará a partir de las $k$ muestras obtenidas, $k>25$ - como regla general se suele tomar $k>20$ ó $k>25$-, tomadas cuando se considera que el proceso está bajo control):

$$
\bar{p}=\frac{1}{k} \sum_{i=1}^{k} \hat{p}_{i}
$$

- En caso de que el tamaño muestral $\left(n_{i}\right)$ sea diferente para cada subgrupo, a la hora de calcular los límites según el modelo de Shewhart, se puede optar por:

1. Los límites varían en función del tamaño de muestra,

2. Si los $n_{i}$ no difieren mucho unos de otros, se podría tomar

$$
n=\frac{1}{k} \sum_{i=1}^{k} n_{i}
$$

3. También se puede optar por tomar un $n$ común e igual al mayor de los $n_{i}$, ya que la amplitud de la franja que indica proceso en estado de control es inversamente proporcional al tamaño de la muestra.

En esta situación de tamaños muestrales diferentes, el estimador para $p$ sería:

$$
\bar{p}=\frac{\sum_{i=1}^{k} n_{i} \hat{p}_{i}}{\sum_{i=1}^{k} n_{i}}
$$

Normalmente se usan límites de control de $3 \sigma$ en el diagrama de control $p$, así como en el resto de gráficos de control por atributos. El uso de límites de control más estrechos hacen que el gráfico de control sea más sensible a pequeños cambios en $p$, pero ello también hace aumentar la probabilidad de que se produzcan falsas alarmas de proceso fuera de control. 


\subsection{2. $\quad$ El gráfico np}

Los gráficos $n p$ se aplican al mismo tipo de procesos que en el caso anterior. La diferencia está en que, en lugar de contabilizar la proporción de unidades defectuosas en una muestra, se considera el número de unidades defectuosas en la muestra, para ello se toman de forma sistemática muestras de tamaño constante a intervalos fijos de tiempo. Este tipo de gráficos permite analizar el número de artículos defectuosos para así poder detectar la posible existencia de causas especiales en el proceso productivo. En cada muestra se evalúa el número de unidades defectuosas, independientemente de que se presenten varios defectos en alguna unidad.

Los principios estadísticos que sirven de base al gráfico de control $n p$ se basan en la distribución Binomial, al igual que en el gráfico $p$ explicado en la sección anterior.

- Sabemos que: $\mu_{X_{i}}=n p$ y $\sigma_{X_{i}}=\sqrt{n p(1-p)}$

- Para cada muestra, se define la variable aleatoria fracción disconforme muestral como: $\hat{p}_{i}=\frac{X_{i}}{n}$. Observar que $\hat{p}_{i}$ seguirá una distribución Binomial con media y desviación típica:

$$
\begin{gathered}
E\left[\hat{p}_{i}\right]=\frac{E\left[X_{i}\right]}{n}=p \\
\operatorname{Var}\left[\hat{p}_{i}\right]=\frac{\operatorname{Var}\left[X_{i}\right]}{n^{2}}=\frac{p(1-p)}{n}
\end{gathered}
$$

- Por tanto, si $n$ es suficientemente grande, se aplica el Teorema Central del Límite y utilizar que, aproximadamente,

$$
n \hat{p}_{i} \longrightarrow N\left(n p, \sqrt{\frac{n p(1-p)}{n}}\right) \text {, si } n \rightarrow \infty
$$


- Para el cálculo de la media y los límites del gráfico de control se aplicarán las siguientes fórmulas:

$$
\begin{gathered}
L C S=n p+z_{\frac{\alpha}{2}} \sqrt{n p(1-p)} \\
\text { Línea central }=p \\
L C I=n p-z_{\frac{\alpha}{2}} \sqrt{n p(1-p)}
\end{gathered}
$$

- Si $p$ es desconocida, se puede estimar (observar que tal estimación se realizará a partir de las $k$ muestras obtenidas, $k>25$, tomadas cuando se considera que el proceso está bajo control) (Montgomery 1991):

$$
\bar{p}=\frac{1}{k} \sum_{i=1}^{k} \hat{p}_{i}
$$

\subsection{Los gráficos $c$ y $u$}

Los gráficos c y u sirven para controlar el número de defectos; el primero exige que el tamaño de muestra sea constante, mientras que este requisito no es necesario en el gráfico u.(Montgomery 1991)

\subsubsection{El gráfico $c$}

Este tipo de gráfico controla la evolución de los defectos presentes en muestras de tamaño constante tomadas a intervalos fijos de tiempo.

El gráfico c está basado en el número total de defectos o de no conformidades en la producción. Los principios estadísticos que sirven de base al gráfico de control $c$ se basan en la distribución de Poisson: 
Denotamos por $c_{i}$ al número de defectos en la muestra i-ésima.

Si llamamos $c$ al valor esperado o valor medio del número de defectos por muestra, entonces:

Se cumplirá que,

$$
c_{i} \longrightarrow P s(c) \approx N(c, \sqrt{c}) \text { si } c>5
$$

Se tendrá que:

$$
\begin{aligned}
& L C S=c+z_{\frac{\alpha}{2}} \cdot \sqrt{c} \\
& \text { Línea central }=c \\
& L C I=c-z_{\frac{\alpha}{2}} \cdot \sqrt{c}
\end{aligned}
$$

Si $c=E\left[c_{i}\right]$ es desconocida, se puede estimar (observar que dicha estimación se realizará a partir de las $k$ muestras obtenidas, tomadas cuando se considera que el proceso está bajo control):

$$
\hat{c}=\bar{c}=\frac{1}{k} \sum_{i=1}^{k} c_{i}
$$




\subsubsection{El gráfico $u$}

Este tipo de gráfico representa los defectos por unidad presentes en muestras tomadas a intervalos fijos de tiempo. Al trabajar en defectos por unidad este gráfico $u$ permite trabajar tanto con muestras de tamaño fijo como variable. Se utiliza cuando en un muestreo como el definido para el gráfico $c$, no puede mantenerse constante el tamaño de la muestra. Debido a ello se trabaja con tasa de defectos por unidad.

Si llamamos $c_{i}$ al número de defectos (u ocurrencias de cierto suceso) en la muestra i-ésima y $n_{i}$ al tamaño de la muestra, el número de defectos por unidad de medida será:

$$
u_{i}=\frac{c_{i}}{n_{i}}=\frac{\text { Numero de defectos en } n_{i} \text { unidades }}{\text { Numero de unidades en la muestra }}
$$

La variable $c_{i}$ es una variable de Poisson de parámetro: $\lambda_{i}=n_{i} \cdot u$

donde $u$ es el número medio de sucesos por unidad. Por tanto:

$$
\begin{gathered}
E\left(c_{i}\right)=\lambda_{i}=n_{i} \cdot u \\
\operatorname{Var}\left(c_{i}\right)=\lambda_{i}=n_{i} \cdot u
\end{gathered}
$$

Por tanto, la variable $u_{i}$ es el cociente de una variable de Poisson y una constante, que es el tamaño de muestra:

$$
u_{i} \equiv \frac{1}{n_{i}} P s\left(n_{i} \cdot u\right) \approx N\left(u, \sqrt{\frac{u}{n_{i}}}\right) \text { si } n_{i} \uparrow \uparrow
$$


El gráfico de control de la variable $u_{i}$ será:

$$
\begin{aligned}
& L C S=u+z_{\frac{\alpha}{2}} \sqrt{\frac{u}{n_{i}}} \\
& \text { Línea central }=\lambda \\
& L C I=u-z_{\frac{\alpha}{2}} \sqrt{\frac{u}{n_{i}}}
\end{aligned}
$$

Si la media $u$ es desconocida, se puede estimar con valores preliminares de $u_{i}$. La media de la distribución del número medio de defectos se estimará con

$$
\bar{u}=\frac{\text { numero total de defectos }}{\text { numero total de unidades }}=\frac{\sum_{i=1}^{k} c_{i}}{\sum_{i=1}^{k} n_{i}}
$$

El gráfico de control u con los límites estimados será

$$
\begin{aligned}
& L C S=\bar{u}+z_{\frac{\alpha}{2}} \sqrt{\frac{\bar{u}}{n_{i}}} \\
& \text { Línea central }=\bar{u} \\
& L C I=\bar{u}-z_{\frac{\alpha}{2}} \sqrt{\frac{\bar{u}}{n_{i}}}
\end{aligned}
$$

El LCI será cero si la fórmula anterior diese un valor negativo. 


\subsection{El muestreo doble}

El muestreo doble es un caso particular del muestreo múltiple que consiste en la toma de decisiones en dos etapas frente a una única etapa habitual en los gráficos clásicos.

La mayor parte de los trabajos que usan este enfoque se orienta al CONTROL POR VARIABLES.

Así, en (Daudin 1992) se propone una nueva metodología: el gráfico de Doble Muestreo para la media DS- $\bar{X}$, consistente en hacer que el plan de muestreo se realice en dos etapas (Double Sampling - DS). Este procedimiento ofrece una eficacia estadística mejor (en términos de ARL- Longitud Promedio de Fuera de Control ${ }^{2}$ ) que el gráfico para la media de Shewhart, sin incrementar el muestreo. Alternativamente, el procedimiento se puede utilizar para reducir el muestreo sin reducir la eficacia estadística. Esta metodología se explica detalladamente en el próximo capítulo de esta Tesis Doctoral.

Hay numerosos artículos posteriores a (Daudin 1992) para gráficos por variables incluyendo el muestreo múltiple (muestreo realizado en dos o más etapas).

En este sentido, por ejemplo, en (He and Grigoryan 2002), explica que los gráficos de control DS- $\bar{X}$ están diseñados para permitir la rápida detección de un pequeño cambio en la media del proceso y ofrece una rápida respuesta en un entorno de fabricación ágil. Sin embargo, los gráficos DS- $\bar{X}$ asumen que la desviación estándar permanece sin cambios durante el curso del control de procesos estadístico. Entonces propone desarrollar un gráfico DS complementario que se puede usar para controlar la variación del proceso ocasionada por

\footnotetext{
${ }^{2}$ Número promedio de puntos que deben representarse gráficamente antes de que un punto indique una condición de fuera de control
} 
cambios en la desviación estándar del proceso. En este artículo se presenta el desarrollo de gráficos DS-s para detectar pequeños cambios en la desviación estándar del proceso de fabricación ágil. La construcción de los gráficos DS- $s$ está basada en los mismos conceptos que la construcción de los gráficos DS- $\bar{X}$ y están formulados como un problema de optimización y resueltos con un algoritmo genético, como vamos a hacer en esta Tesis Doctoral. En este artículo se compara la eficiencia del gráfico DS- $s$ con la de los gráficos de control $s$ tradicionales. Los resultados muestran que los gráficos DS- $s$ pueden ser una alternativa preferible en la detección de pequeños cambios que los gráficos de control $s$ tradicionales.

En (He, Grigoryan, and Sigh 2002) profundiza en el doble muestreo propuesto por (Daudin 1992) y desarrolla gráficos de triple muestreo (TS), en los que concluye, tras comparar con el DS- $\bar{X}$ que son más eficientes en términos de minimizar el tamaño de muestra medio.

En otro artículo posterior de los mismos autores (He and Grigoryan 2003), realizan mejoras a su propuesta anterior para su aplicación no esté limitada a tamaños de muestra pequeños. En todos sus estudios han empleado algoritmos genéticos para su resolución.

Muchos autores están trabajando en el muestreo múltiple para gráficos por variables. Como muestra los artículos (Costa and Machado 2007), (Costa and Rahim 2006), (Costa and De Magalhâes 2005), (Costa, De Magalhâes, and Epprecht 2005), (Costa and Rahim 2004), (Zimmer, Montgomery, and Runger 2000), (Zimmer, Montgomery, and Runger 1998), (Che and Chen 2000), (Carot, Jabaloyes, and Carot 2002), (Costa 2001) y (Chan et al. 2003). En todos ellos se hace referencia al artículo (Daudin 1992), que es la base sobre la que realizan modificaciones y nuevas aportaciones. El artículo (Daudin 1992) está ampliamente detallado en la sección 5.1.

Por contra, en la revisión bibliográfica realizada se han encontrado escasos 
artículos que hagan referencia a la posibilidad de realizar múltiples etapas de muestreo en GRÁfICOS DE CONTROL POR ATRIBUTOS.

En (Jolayemi 2002), que se trata de un artículo para el diseño de gráficos $n p$ con tres y cuatro regiones de control, se dan ejemplos numéricos para ilustrar el modelo y para estudiar las propiedades de los gráficos. Los resultados de los ejemplos numéricos muestran que los cambios en los valores específicos de muchos de los riesgos y potencias asociadas con el gráfico- particularmente los clasificados como riesgos críticos- afectan a los valores de los parámetros de diseño de los gráficos. Se dibujan las curvas de OC y potencia para algunos de los ejemplos numéricos, que muestran que la potencia discriminatoria de un gráfico $n p$ con múltiples regiones de control depende de los valores de los parámetros de diseño y el número de regiones de control- cuantas más regiones de control, menor potencia discriminatoria del gráfico, y viceversa. 


\section{Capítulo 4}

\section{Algoritmos Genéticos}

\subsection{Origen.}

\subsubsection{Introducción}

En la tecnología y en la ciencia, los algoritmos genéticos se han utilizado como algoritmos adaptativos para la resolución de problemas prácticos tales como modelos computacionales de sistemas evolutivos naturales.

Los algoritmos genéticos (AG) fueron inventados por John Holland, de la Universidad de Michigan en los años 60, y fueron desarrollados por Holland y sus alumnos en los años 60 y 70 . Su objetivo era estudiar formalmente el fenómeno de adaptación tal y como ocurre en la naturaleza y desarrollar maneras por las cuales se pudiera importar a sistemas de computación los mecanismos de la adaptación natural. No pretendía diseñar algoritmos para resolver problemas específicos, aunque el desarrollo de sus estudios haya conducido a este punto. 
Los AG son, simplificando,algoritmos de optimización, es decir, tratan de encontrar la mejor solución a un problema dado entre un conjunto de soluciones posibles. Los mecanismos de los que se valen los AG para llevar a cabo esta búsqueda pueden verse como una metáfora de los procesos de evolución biológica.

El uso de la inteligencia artificial es cada vez mayoritario. Se definen como:

Algoritmos de búsqueda basados en los mecanismos de selección natural y genética natural. Combinan la supervivencia de los más compatibles entre las estructuras de cadenas, con una estructura de información ya aleatorizada, intercambiada para construir un algoritmo de búsqueda con algunas de las capacidades de innovación de la búsqueda humana

(Goldberg 1989)

Los Algoritmos Genéticos difieren de los métodos tradicionales de búsqueda y optimización, en cuatro cuestiones esenciales:

1. Trabajan con un código del conjunto de parámetros, no con el conjunto mismo (necesitan que el conjunto de parámetros del problema de optimización esté codificado en cadenas finitas sobre un determinado alfabeto). Por trabajar a nivel de código, y no con las funciones y sus variables de control, como los otros métodos, son más difíciles de engañar.

2. Buscan una población de puntos, no un único punto. Manteniendo una población de puntos muestrales bien adaptados, se reduce la probabilidad de caer en una cima falsa. 
3. Emplean la función objetivo, no necesitan derivadas ni otra información complementaria, tan difícil a veces de conseguir. De este modo ganan en eficiencia y en generalidad.

4. Se valen de reglas de transición estocásticas, no deterministas. Los Algoritmos Genéticos se valen de operadores aleatorios para guiar la búsqueda de los mejores puntos; puede parecer extraño, pero la Naturaleza está llena de precedentes al respecto.

El primer y más importante punto es que los algoritmos genéticos son intrínsecamente paralelos. La mayoría de los otros algoritmos son en serie y sólo pueden explorar el espacio de soluciones hacia una solución en una dirección al mismo tiempo, y si la solución que descubren resulta subóptima, no se puede hacer otra cosa que abandonar todo el trabajo hecho y empezar de nuevo. Sin embargo, ya que estos algoritmos tienen descendencia múltiple, pueden explorar el espacio de soluciones en múltiples direcciones a la vez. Si un camino resulta ser un callejón sin salida, pueden eliminarlo fácilmente y continuar el trabajo en direcciones más prometedoras, dándoles una mayor probabilidad en cada ejecución de encontrar la solución.

Debido a este paralelismo que les permite evaluar implícitamente muchos esquemas a la vez, los algoritmos genéticos funcionan especialmente bien resolviendo problemas cuyo espacio de soluciones potenciales es realmente grande -mucho para hacer una búsqueda exhaustiva en un tiempo razonable. Así, el algoritmo genético puede dirigirse hacia el espacio de posibles soluciones con los individuos más aptos y encontrar el mejor de ese grupo. En el contexto de los algoritmos evolutivos, esto se conoce como teorema del esquema, y es la ventaja principal de los algoritmos genéticos sobre otros métodos de resolución de problemas. (Goldberg 1989)

Afortunadamente, el paralelismo implícito de estos algoritmos les permite superar incluso un enorme número de posibilidades, y encontrar con 
éxito resultados óptimos o muy buenos en un corto periodo de tiempo, tras muestrear directamente sólo regiones pequeñas del vasto espacio.

Otra ventaja notable de los algoritmos genéticos es que se desenvuelven bien en problemas con un paisaje adaptativo complejo -aquéllos en los que la función de aptitud es discontinua, ruidosa, cambia con el tiempo, o tiene muchos óptimos locales. La mayoría de los problemas prácticos tienen un espacio de soluciones enorme, imposible de explorar exhaustivamente; el reto se convierte entonces en cómo evitar los óptimos locales -soluciones que son mejores que todas las soluciones próximas a ella, pero que no son mejores que otras soluciones distintas situadas en algún otro lugar del espacio de soluciones. Muchos algoritmos de búsqueda pueden quedar atrapados en los óptimos locales: si llegan a lo alto de una colina del paisaje adaptativo, descubrirán que no existen soluciones mejores en las cercanías y concluirán que han alcanzado la mejor de todas, aunque existan picos más altos en algún otro lugar del mapa.

Por otra parte, los algoritmos genéticos comienzan con una mente abierta, como sus decisiones están basadas en la aleatoriedad, todos los caminos de búsqueda posibles están abiertos teóricamente a un algoritmo genético; en contraste, cualquier estrategia de resolución de problemas que dependa de un conocimiento previo, debe inevitablemente comenzar descartando muchos caminos a priori, perdiendo así cualquier solución novedosa que pueda existir (Koza 1999). Los algoritmos genéticos, al carecer de ideas preconcebidas basadas en creencias establecidas sobre cómo deben hacerse las cosas o sobre lo que de ninguna manera podría funcionar, no tienen este problema. De manera similar, cualquier técnica que dependa de conocimiento previo fracasará cuando no esté disponible tal conocimiento, pero, los algoritmos genéticos no se ven afectados negativamente por la ignorancia (Goldberg 1989). Mediante sus componentes de paralelismo, cruzamiento y mutación, pueden viajar extensamente por el paisaje adaptativo, explorando regiones que algoritmos 
producidos con inteligencia podrían no haber tenido en cuenta, y revelando potencialmente soluciones de asombrosa e inesperada creatividad que podrían no habérseles ocurrido nunca a los diseñadores humanos. Los algoritmos evolutivos no están enterados ni preocupados de si una solución va en contra de las creencias establecidas -sólo de si funciona.

Aunque los algoritmos genéticos han demostrado su eficiencia y potencia como estrategia de resolución de problemas, no son la panacea. Tienen ciertas limitaciones; como que la función objetivo debe representar ciertamente la aptitud de una solución: También se ha de elegir bien los parámetros de dicho algoritmo genético, que se encuentran ampliamente explicados más adelante, para evitar efectos como la convergencia prematura.

\subsubsection{Descripción}

Los algoritmos genéticos son, a grosso modo, un método de optimización: dada una función objetivo, el AG lleva a cabo una búsqueda en el espacio de soluciones intentando encontrar la solución que minimiza dicha función objetivo. Por lo tanto, los algoritmos genéticos están indicados para resolver todo tipo de problemas que pueden ser expresados como un problema de optimización: basta con encontrar la representación adecuada para las soluciones y la función a optimizar.

Básicamente, los algoritmos genéticos funcionan como sigue: dada una población de soluciones, y en base al valor de la función objetivo para cada uno de los individuos (soluciones) de esa población, se seleccionan los mejores individuos (los que minimizan la función objetivo) y se combinan para generar otros nuevos. Este proceso se repite cíclicamente ${ }^{1}$.

\footnotetext{
${ }^{1}$ Hasta que se cumple un criterio de parada (la solución es aceptable o se ha sobrepasado un límite de iteraciones)
} 
Como se puede ver, el proceso es similar al que se da en la naturaleza: una serie de individuos compiten por su supervivencia; los mejor adaptados al medio los que optimizan la función objetivo sobreviven y tienen más posibilidades de aparearse, transmitiendo así parte de su material genético mejor adaptado a las generaciones siguientes. Así, generación tras generación, la especie consigue una mayor y mejor adaptación al medio en el que vive.

Como ya se ha dicho, los algoritmos genéticos están inspirados en la naturaleza, en la evolución de las especies. Por ello, para comprender su funcionamiento es aconsejable conocer primero cómo funcionan los mecanismos de la evolución desde un punto de vista meramente biológico.

\subsection{Implementación}

Se ha utilizado GAlib, que es una librería de $\mathrm{C}++$ que ofrece un conjunto de objetos de algoritmos genéticos al programador de aplicaciones. Con GAlib se puede añadir optimización de algoritmos genéticos al programa utilizando cualquier representación de información y selección, cruce, mutación, escalado, reemplazamiento y métodos de terminación estándares o particulares.

La codificación empleada ha sido la propia codificación natural del valor de la variable, sin proceder a una conversión a codificación binaria, y además GAlib permite esta opción, que reduce costes computacionales del algoritmo. La codificación real se lleva a cabo mediante la organización de los parámetros a optimizar en un array de variables $x$ reales e independientes. GAlib tiene la definición de este array donde se establecen las restricciones explícitas que se apliquen a cada variable real $x_{i}$.

En los últimos años existe una tendencia a utilizar otros tipos de codifi- 
caciones distintas a la binaria. Esto no significa que la codificación binaria no se deba utilizar, sino que la codificación que se ajusta de forma natural al problema probablemente obtendrá mejores resultados y será más fácil de implementar.

Los pasos a seguir por un algoritmo genético son los siguientes (Martorell et al. 2000):

\section{Inicialización}

El primer paso en la cadena evolutiva es la creación de una población inicial $(\mathrm{g}=0)$ de un determinado tamaño, que no se trata como las otras, sino que es directamente evaluada. La selección de un número adecuado de individuos en la población, popsize, es un aspecto muy importante a tener en cuenta,debido a que afectará al comportamiento del algoritmo. Por consiguiente, con una población pequeña corremos el riesgo de no cubrir todo el espacio de búsqueda entero, y el algoritmo genético podría converger en una solución prematuramente. Por el contrario, trabajar con muchos individuos de una población podría suponer un coste computacional adicional e innecesario. En nuestro caso, se han realizado diferentes ensayos para obtener un tamaño apropiado de población, y se ha determinado que en la mayoría de los casos, una población de entre 120 y 200 individuos da buenos resultados de optimización.

Normalmente, se genera la población inicial usando un método aleatorio, pero también se puede obtener mediante la aplicación de diferentes técnicas heurísticas, dependiendo del problema particular.

\section{Selección}

Después de la inicialización, el algoritmo emprende el ciclo evolutivo de generación en generación con la selección de algunos individuos de 
la población base para ser los padres en la etapa de reproducción. Se ha escogido la selección por competición o torneo de todos los métodos que tiene GALib para seleccionar individuos. Este criterio es una extensión del método de la ruleta, o selección simple, que es un método de muestreo estocástico que toma los individuos simulando una ruleta. El selector de torneo utiliza la ruleta para seleccionar un grupo de dos o más individuos de la población base y entonces elige un individuo con el mejor marcador dentro de cada grupo. Este proceso se repite hasta que se alcanza el número requerido de individuos seleccionados. Entonces, siguiendo las leyes de la Naturaleza, los mejores individuos deberían reproducirse más, para así generar más descendientes que contengan parte de su información genética. Por lo tanto, prevalecerán principalmente las mejores soluciones en la descendencia y en su información genética, que pasará a la siguiente generación.

3. Reproducción

El objetivo de la reproducción es crear nuevos individuos usando operadores genéticos. Un algoritmo genético utiliza, por lo menos, dos operadores genéticos para la reproducción: cruce y mutación.

a) Cruce. El proceso de cruce se aplica a la población resultante del proceso de selección. Se empareja de forma aleatoria a todas las soluciones de la población. Cada una de estas parejas se reproduce con una determinada probabilidad de cruce, que es fija para toda la población. Esta probabilidad suele oscilar entre 0.6 y 0.99. Para nuestros ensayos se ha fijado $P_{\text {cruce }}=0.9$, ya que en los experimentos preliminares realizados nos ofrecía una mejor optimización. Si la pareja no se reproduce, cada uno de los individuos queda inalterado en la población, si por el contrario se cruzan, se generan dos nuevas soluciones, que heredan una combinación de características de sus progenitores y que reemplazan a éstos. Así, siempre 
quedará el tamaño de población invariable. Los mejores individuos son los que cruzan de forma más activamente en el cruce, ya que aparecen más veces en la población, y así tienen más posibilidades de pasar su material genético a las siguientes generaciones. Como no todos los individuos se cruzan, en una misma población puede haber individuos de distintas generaciones. El proceso de cruce no debe consistir solamente en combinar soluciones para obtener nuevas soluciones, sino que esa combinación debe ser verdaderamente beneficiosa (Falkenauer 1998). Se ha escogido el cruce 1-punto de todos los métodos disponibles en GALib, que es un método muy sencillo utilizado ampliamente que ofrece buenos resultados. Este método consiste en seleccionar aleatoriamente una posición en el genoma de ambos padres por la cual cada genoma de la pareja se cortará en dos piezas. Entonces, se mezclan las dos partes para obtener los dos hijos nuevos.

b) Mutación. Cuando se termina el proceso de cruce, se inicia el de mutación. El objeto de esta etapa es introducir variabilidad en la población, introduciendo en algunos individuos características nuevas, o características que se habían dado en la población, pero que pudieron haberse perdido a lo largo del proceso de evolución. Por tanto, se pretende asegurar que no haya ninguna solución de todo el espacio que tenga probabilidad nula de ser examinada. En algunos estudios se sugiere que tome valores en el intervalo $[1 / n, 1]$, siendo $n$ la longitud del vector de variables. En nuestro caso, el genoma tiene un tamaño de 8 variables, por lo que el valor de la probabilidad de mutación debe estar comprendido entre [0.125,1], y se ha escogido $P_{m u t}=0.2$ (Martorell et al. 2000).

4. Evaluación

Después de la reproducción, el siguiente paso en la evolución corres- 
ponde a la evaluación de los nuevos individuos, de acuerdo con la función objetivo adoptada en un principio.

\section{Penalización}

Una vez calculados los valores de la función objetivo para los nuevos individuos, el algoritmo comprueba si las restricciones implícitas se satisfacen, penalizando a los individuos que violan una o más de dichas restricciones. En nuestro caso no se han incluido penalizaciones, sino que el algoritmo genético tiene suficiente información en la función objetivo para hacer la evolución correspondiente de los individuos y alcanzar así una solución óptima.

6. Escalado

Para evitar una convergencia prematura en generaciones tempranas, o un estancamiento en individuos mediocres, se procede al escalado que controla la presión selectiva. De las posibles funciones de GAlib se ha elegido la función de sigma truncation, ya que el escalado lineal(Martorell et al. 2000) no funcionaba adecuadamente con nuestro problema. Además, la función sigma truncation mantiene la presión de selección relativamente constante y restringe los efectos que un único máximo local puede tener sobre toda la población (Goldberg 1989).

7. Reemplazamiento

Después de escalar todos los individuos de la población, el algoritmo experimenta un reemplazamiento. Se realiza un ranking con todos los individuos que hay en la población más los individuos seleccionados por medio del selector de torneo del paso 2, de acuerdo con su marcador de salud, y se eliminan la misma cantidad de individuos seleccionados en el paso 2, de entre los peores de la lista, así se restituye el tamaño original de la población. 


\section{Terminación}

El ciclo evolutivo se repite a través de las generaciones hasta que se cumple el criterio de terminación. Este criterio de terminación especifica las condiciones que se deben satisfacer para considerar que se ha encontrado la mejor solución, o de otro modo se acepta que el algoritmo genético ha evolucionado a un número máximo de generaciones. En nuestro caso particular se ha marcado un límite de número de generaciones (150000 en este caso, pero el software permite seleccionar el número máximo deseado por parte del usuario), aunque si el algoritmo converge antes de alcanzar el número máximo de generaciones, se detiene. Esto conlleva otra implicación, si la terminación es por número de generaciones, puede que la solución obtenida no sea la óptima, pero si una solución que mejore las prestaciones de partida.

Haciendo uso de GALib, se ha realizado la programación en $\mathrm{C}++$ de las funciones y procedimientos necesarios para resolver nuestro problema de optimización. El listado con estas funciones y procedimientos de nuestro problema a resolver está en el anexo II. Con la ayuda del programa realizado se han obtenido resultados, que se muestran en los siguientes capítulos.

\subsection{Software desarrollado}

La información aportada por el usuario al programa es la relativa a los parámetros del Algoritmo Genético, así como los referentes al gráfico clásico $u$ cuya potencia se pretende mejorar.

El software realiza simulaciones con posibles soluciones para seleccionar una de ellas que mejore el valor de la potencia en $u_{1}$ para el gráfico $D S-U$ propuesto, comparándose con el valor de la potencia en $u_{1}$ en el gráfico $u$. 
Todo ello siendo la potencia en $u_{0}$ igual, o muy similar -la distribución de Poisson es discreta, por lo que su función de distribución no es contínua- en ambos casos, y siendo el tamaño medio de muestra del gráfico $D S-U$ igual o menor que el tamaño requerido para el gráfico $u$.

Para resolver el problema planteado se ha desarrollado una aplicación informática con un entorno MS-Windows, en el cual el usuario puede seleccionar los parámetros de entrada del algoritmo genético que va a ejecutarse para encontrar una solución buena.

A continuación se muestran todas las opciones disponibles en la aplicación informática desarrollada para esta Tesis Doctoral. La mayoría están explicadas en este capítulo, y para una información más detallada y concreta se puede consultar las siguientes referencias bibliográficas, entre otras: (Goldberg 1989), (Falkenauer 1998), (Bäck and Schwefel 1993), (Bäck 1998) y (Bäck 1996).

La interfaz de interacción con el usuario permite seleccionar los siguientes parámetros, agrupados en tres apartados:

1. Los operadores genéticos y los tipos que se pueden seleccionar son:

$\begin{array}{cccc}\text { SELECCIÓN } & \text { CRUCE } & \text { ESCALADO } & \text { MUTACIÓN } \\ \text { DS } & \text { Uniform } & \text { No Scaling } & \text { Flip } \\ \text { Rank } & \text { Even Odd } & \text { Linear } & \text { Swap } \\ \text { Roulette Wheel } & \text { One Point } & \text { Sigma Truncation } & \text { Gaussian } \\ \text { SRS } & \text { Two Point } & \text { Power Law } & \\ \text { Tournament } & \text { Partial Match } & \text { Sharing } & \\ \text { Uniform } & \text { Order } & & \\ & \text { Cycle } & & \end{array}$


2. En cuanto a los parámetros del Algoritmo Genético modificables a través del interfaz del programa son:

- Tamaño de la población : Número de individuos de la población.

- Número de generaciones : El criterio de parada escogido para esta aplicación es por número de generaciones o bien por convergencia del algoritmo.

- Probabilidad de cruce : Probabilidad de que una pareja de soluciones se reproduzca.

- Probabilidad de mutación : Probabilidad de introducir variaciones o mutaciones en un individuo.

- Probabilidad de convergencia : Probabilidad de que las soluciones lleven a un mismo individuo.

3. Los parámetros del gráfico u clásico son los siguientes:

- Valor del número de defectos por unidad muestreada cuando el proceso está bajo control: $u_{0}$

- Valor del número de defectos por unidad muestreada cuya potencia de detección se pretende mejorar : $u_{1}$

- Tamaño de muestra: $n$

- Límite de Control Superior: LCS

- Límite de Control Inferior: LCI

- Error de Primera Especie: $\alpha_{\text {gráfico u }}$

Podemos ver la disposición de esta información en la pantalla de toma de datos de la aplicación desarrollada a tal efecto en la figura 4.1. 


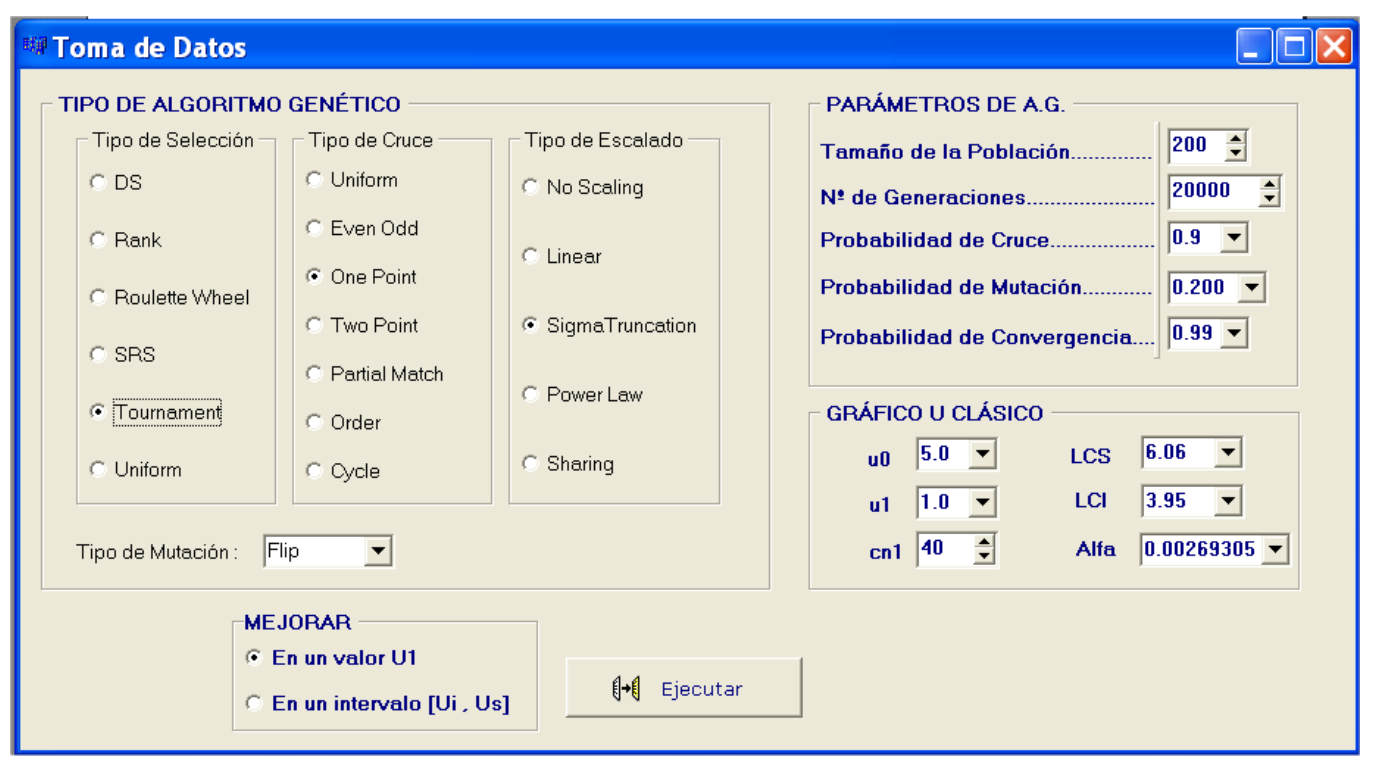

Fig. 4.1: Pantalla de toma de datos 


\section{Capítulo 5}

\section{Propuesta de mejora del gráfico u: El gráfico DS-U}

\subsection{Metodología de Daudin}

J.J. Daudin (Daudin 1992) propuso un nuevo procedimiento, el gráfico de Doble Muestreo para la media DS- $\bar{X}$, que es la forma de hacer que el plan de muestreo se realice en dos etapas (Double Sampling - DS). Este procedimiento ofrece una eficacia estadística mejor (en términos de ARLLongitud Promedio de Salida de Control) que el gráfico para la media de Shewhart, sin incrementar el muestreo. Alternativamente, el procedimiento se puede utilizar para reducir el muestreo sin reducir la eficacia estadística. En contraste con la metodología VSI (Variable Sampling Interval- Intervalo de Muestreo Variable), para el procedimiento de DS propuesto, se asume que se pueden tomar dos muestras sucesivas sin diferencia de tiempo y, por lo tanto, vienen de la misma distribución de la probabilidad. En esta última afirmación se ha de tener en cuenta que se puede recoger una muestra principal de un 
total de $\left(n_{1}+n_{2}\right)$ unidades, todo al mismo tiempo, analizar las primeras $n_{1}$ unidades, y después decidir si analizar las $n_{2}$ unidades restantes. Así, es posible utilizar un gráfico de DS incluso cuando se requiere un tiempo largo para analizar o medir la muestra. Como con todos los procedimientos habituales de gráficos de control, también se asume que los valores de la muestra son independientes.

Cuando se aumenta el tamaño de muestra para el gráfico de control Shewhart $\bar{X}$, mejora su capacidad de detectar cambios y desviaciones sin aumentar la ocurrencia de falsas alarmas. Sin embargo, el aumento del tamaño de muestra tiene algunas desventajas. Aumenta no sólo los costes del muestreo y de la medida, sino que también aumenta el tiempo de reacción cuando se requiere una cantidad de tiempo relativamente grande para analizar o para medir las unidades de la muestra, y retrasa así la corrección de una causa asignable. Los investigadores han hecho esfuerzos de mejorar el gráfico de Shewhart $\bar{X}$ sin el aumento de la cantidad de muestreo. Los ejemplos de dichos gráficos mejorados son el uso de las reglas suplementarias de funcionamiento (Champ and Woodall 1987), el uso de los intervalos de muestreo variables (VSI) (Reynolds Jr et al. 1988), de los gráficos EWMA, y CUSUM.

El diagrama de flujo del gráfico u clásico (figura 5.1) muestra que éste transcurre en una única etapa, en la que se decide en función de la proporción de defectos que genera el proceso en el momento de tomar una muestra de tamaño $n_{i}$.

Se calcula el número de defectos por unidad $u$, y si pertenece al intervalo de los límites de control, se acepta que el proceso está bajo control, de lo contrario, se entiende que el proceso está fuera de control, y habría que tomar las medidas pertinentes.

Aunque el gráfico $u$ original permite trabajar con tamaño de muestra variable, y así está recogido en la figura 5.1, para el resto de este estudio se 
va a considerar que $n_{i}=n \forall i$, es decir, que el tamaño de muestra es constante.

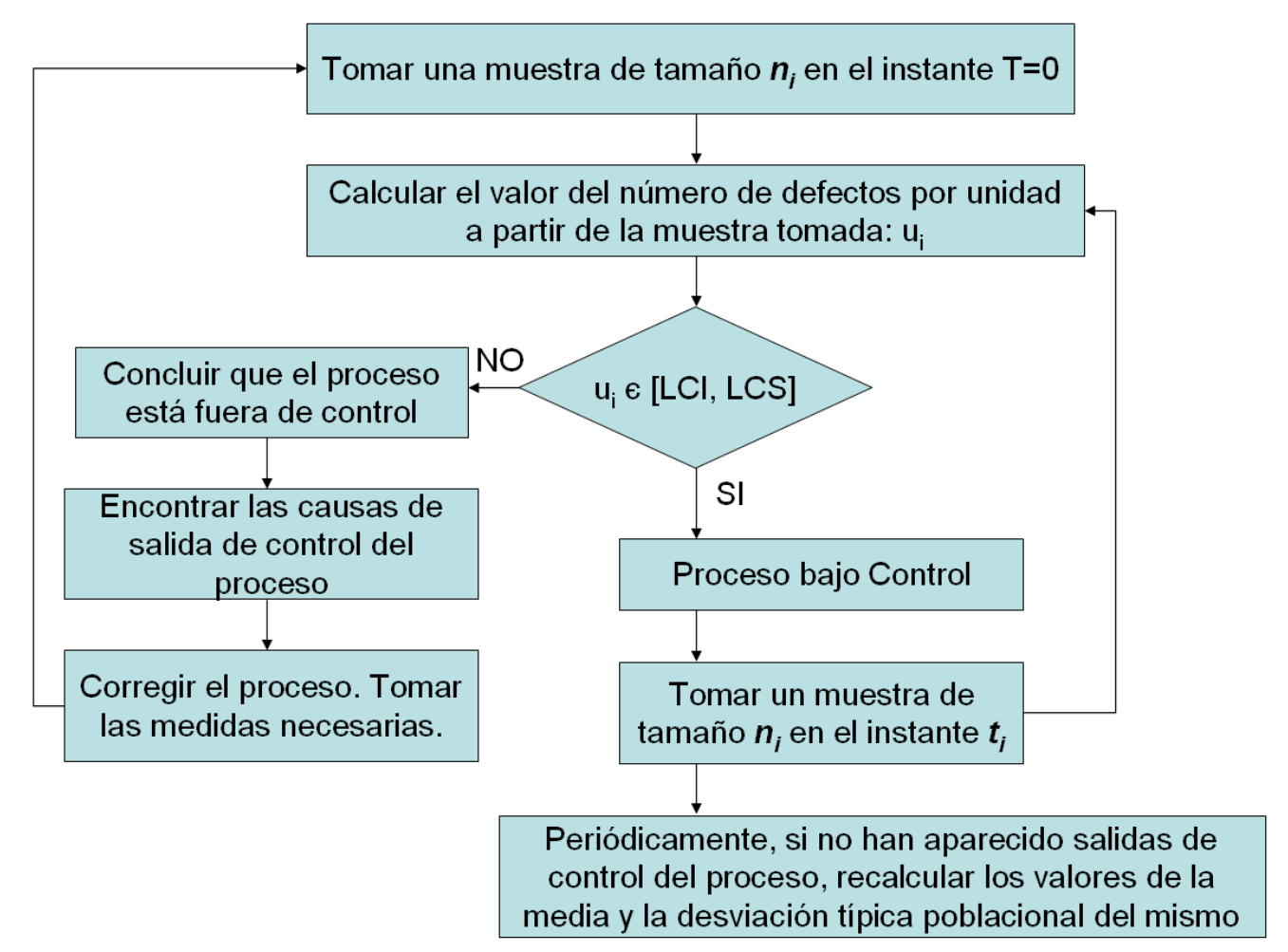

Fig. 5.1: Diagrama de flujo del gráfico u

El diagrama de flujo del nuevo gráfico $D S-U$ se muestra en la figura 5.2. Para esta nueva propuesta de gráfico, se han fijado dos etapas. La primera etapa tiene una Región de Control 1, y dentro de ella hay una Región de Atención, que es la que fija si se requiere el estudio de la segunda muestra para tomar una decisión sobre si el proceso está bajo control o no. Si hay que analizar la segunda muestra, se tiene una Región de Control 2, en la que ya se decide de forma definitiva sobre la interpretación de las muestras recogidas. 


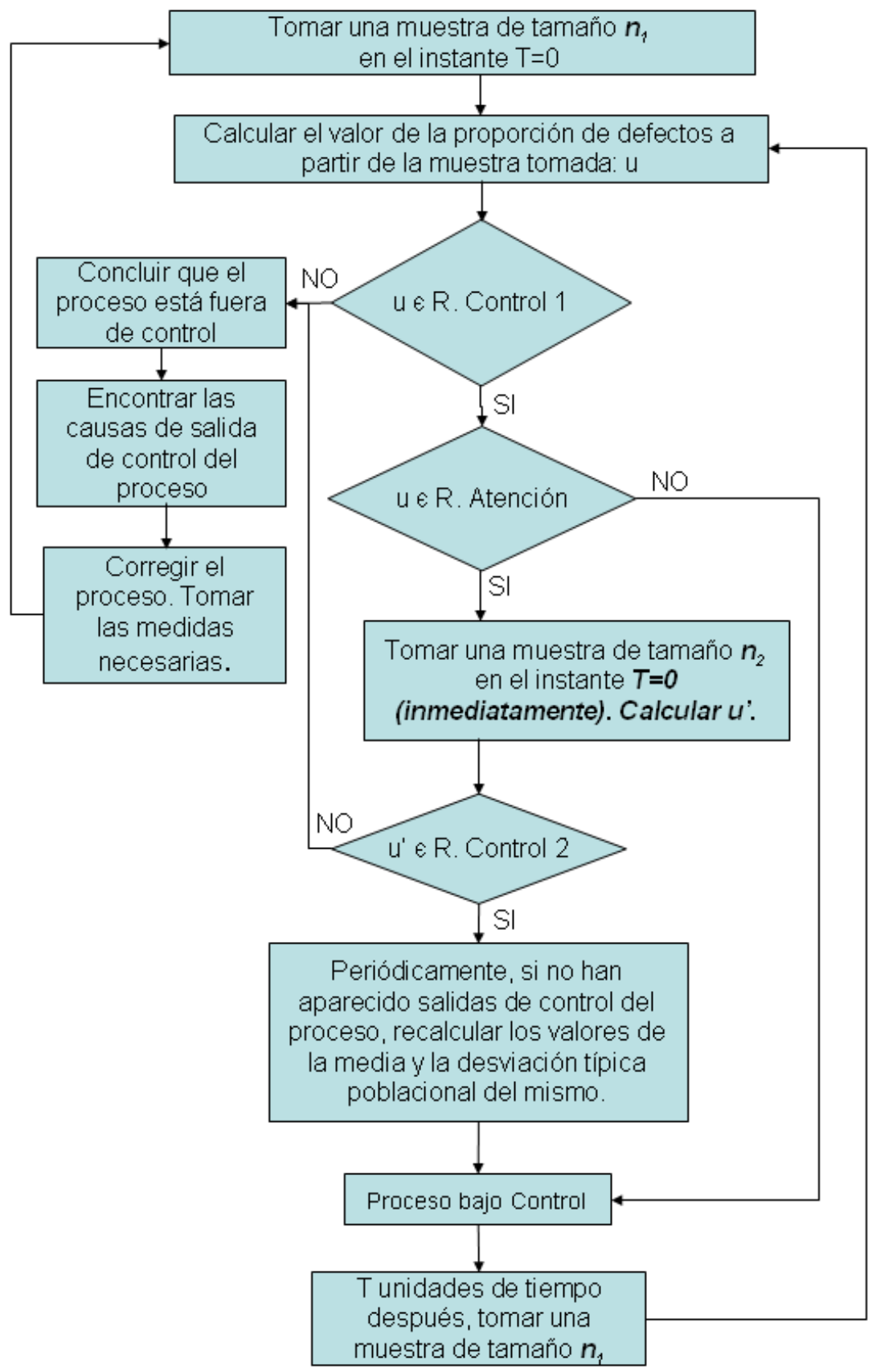

Fig. 5.2: Diagrama de flujo del gráfico $D S-U$ 
El gráfico $D S-U$ que se propone en este trabajo de investigación está representado en la figura 5.3 con la nomenclatura de dicho gráfico de control que se va a emplear a lo largo de esta Tesis Doctoral.

Primero se toma una muestra de tamaño $n_{1}$ en el instante $t_{1}$. Seguidamente se calcula a partir de una muestra el valor de $u$, siendo $u$ el número medio de defectos por unidad de inspección. Entonces se pregunta si se encuentra dentro de los límites de control establecidos: [LCI, LCS].

Si la respuesta es no, se concluye que el proceso está fuera de control (punto 1), y en ese caso se han de encontrar las causas de la salida de control del proceso, y seguidamente corregirlo tomando las medidas necesarias.

Si en cambio sí se encuentra dentro de los límites de control establecidos, se averigua si se encuentra dentro de la región de atención marcada: $[L A S, L C S] \cup[L C I, L A I]$.

Si la respuesta es negativa porque están en el intervalo $[L A I, L A S]$ (punto 2), entonces se concluye que el proceso es estable y bajo control, con lo que $T$ unidades de tiempo después se volvería a tomar una muestra de tamaño $n_{1}$.

Si por el contrario se encuentra en la zona de atención (punto 3), tomaremos una muestra de tamaño $n_{2}$ inmediatamente después, y se calcula el parámetro $u^{\prime}$ - número medio de defectos por unidad de inspección en la segunda etapa. Después se considerará si se encuentra dentro de los límites de control nuevos marcados al efecto para un segundo muestreo. Si es que no (punto 5), el proceso está fuera de control y se tendrá que revisar; o bien, si es que sí (punto 4), el proceso es estable y bajo control, pasando de nuevo a la etapa 1.

En esta etapa 1, el punto 6 indica que el proceso es estable y bajo control. 


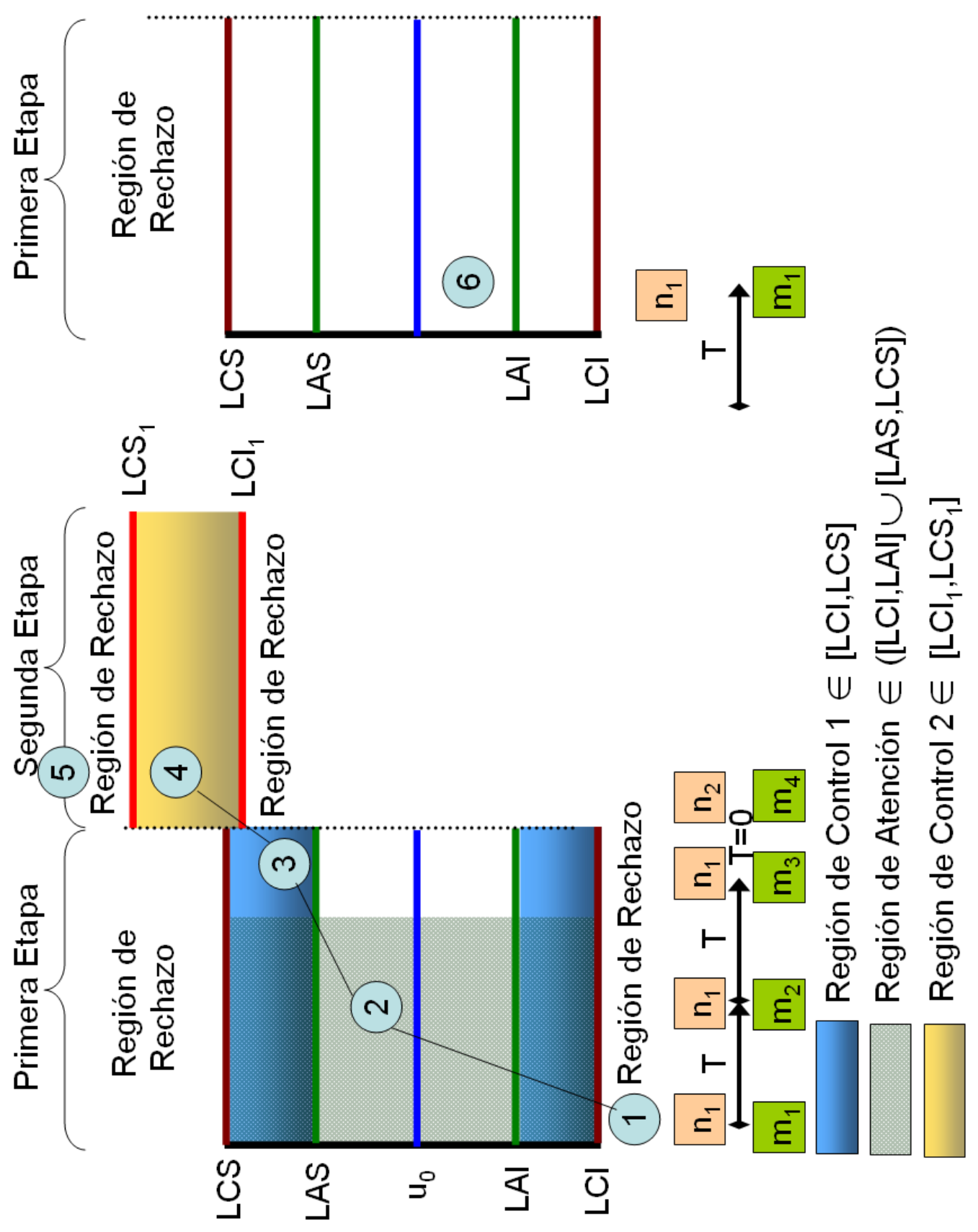

Fig. 5.3: Gráfico de control $D S-U$ 


\subsection{Formulación del problema}

Para el cálculo de los límites de control y de atención de las dos etapas, y de los tamaños de muestra $n_{1}$ y $n_{2}$ que mejoren la curva de potencia, se ha hecho uso de los algoritmos genéticos que nos permiten llegar a una buena solución.

En esta sección se muestra la formulación matemática del problema. En ella se ha empleado la distribución de Poisson, sin aplicar la aproximación a la distribución Normal, aunque en la práctica usual del Control de la Calidad no se utiliza este método y se suele aproximar. En el caso que nos ocupa, dado que se busca la información más precisa disponible para realizar una comparación lo más exhaustiva posible, se ha empleado la formulación exacta.

Al tratar de maximizar $\operatorname{Pot}_{D S-U}-P_{0} t_{u}$, se exige que el error de primera especie sea igual en ambos gráficos:

$$
\alpha_{\text {gráfico u }} \approx \alpha_{\text {gráfico DS-U }}
$$

Asímismo, en cuanto al tamaño de muestra, se establece que el tamaño medio de muestra obtenido en las dos etapas del gráfico $D S-U$ debe ser menor o igual que el tamaño de muestra establecido en el gráfico $u$ :

$$
E[n]_{\text {gráfico DS-U }} \leq n_{\text {gráfico u }}
$$

Se calcularán los límites de control del nuevo gráfico $D S-U$ (de la primera etapa: $L C I, L A I, L A S$ y $L C S$, y de la segunda etapa: $L C I 1$ y $L C S 1)$ de modo que se maximice $\operatorname{Pot}_{D S-U}-$ Pot $_{u}$ para un valor $u_{1}$ o en un intervalo $\left[u_{\text {inferior }}, u_{\text {superior }}\right]$.

Se obtendrán estos valores a partir del software desarrollado empleando algoritmos genéticos, en el cual se han introducido los modelos correspondien- 
tes a los gráficos u clásico y $D S-U$. Las expresiones empleadas se detallan en las siguientes subsecciones.

\subsubsection{Gráfico $u$}

El error de tipo I, $\alpha$ o error de primera especie es ${ }^{1}$ :

$$
\begin{aligned}
\alpha_{\text {gráfico u }}= & P\left(u_{i} \notin[L C I, L C S] \mid u=n \cdot c p_{0}\right)= \\
= & 1-P\left(L C I \leq u_{i} \leq L C S \mid u=n \cdot c p_{0}\right) \\
\alpha_{\text {gráfico u }}= & 1-\sum_{i=c e i l(c L C I \cdot n)}^{f l o o r(c L C S \cdot n)} \frac{e^{-n \cdot c p_{0}} \cdot\left(n \cdot c p_{0}\right)^{i}}{i !}
\end{aligned}
$$

donde $n$ es el tamaño de muestra, $c L C S$ es el límite de control superior del gráfico, $c L C I$ es el límite de control inferior del gráfico y $c p_{0}$ es la proporción de defectos cuando el proceso está bajo control. Se tiene que: $c n \cdot c p_{0}=u_{0}$.

De forma análoga se calcula la potencia:

$$
\operatorname{POT}_{\text {gráfico u }}\left(c p_{1}\right)=(1-\beta)_{u}=1-\sum_{i=c e i l(c L C I \cdot c n)}^{\text {floor }(c L C S \cdot c n)} \frac{e^{-c n \cdot c p_{1}} \cdot\left(c n \cdot c p_{1}\right)^{i}}{i !}
$$

donde $c n$ es el tamaño de muestra, $c L C S$ es el límite de control superior del gráfico, $c L C I$ es el límite de control inferior del gráfico y $c p_{1}$ es la proporción de defectos cuando el proceso está fuera de control.

\footnotetext{
${ }^{1}$ En algunas expresiones de esta Tesis Doctoral se ha empleado notación correspondiente a lenguaje de programación, tal como ceil() y floor(), que corresponden respectivamente al redondeo superior y redondeo inferior de los números que llevan entre paréntesis.
} 


\subsubsection{Gráfico DS-U}

Para el nuevo gráfico $D S-U$ se han aplicado las siguientes expresiones siguiendo la nomenclatura del gráfico explicada en la figura 5.3, y que se resume en la tabla 5.1

\begin{tabular}{l|l}
\hline$L C I$ & Límite de Control Inferior de la primera etapa \\
\hline$L A I$ & Límite de Atención Inferior de la primera etapa \\
\hline$L A S$ & Límite de Atención Superior de la primera etapa \\
\hline$L C S$ & Límite de Control Superior de la primera etapa \\
\hline$L C I_{1}$ & Límite de Control Inferior de la segunda etapa \\
\hline$L C S_{1}$ & Límite de Control Superior de la segunda etapa \\
\hline$n_{1}$ & Tamaño de muestra de la primera etapa \\
\hline$n_{2}$ & Tamaño de muestra de la segunda etapa \\
\hline$p_{0}$ & Proporción de defectos en el proceso bajo control \\
\hline$p_{1}$ & Proporción de defectos en el proceso fuera de control \\
\hline
\end{tabular}

Tabla 5.1: Nomenclatura del gráfico $D S-U$

El error de tipo I, o $\alpha$, se puede obtener de forma intuitiva a partir del gráfico 5.4, sabiendo que:

$\alpha=P($ Rechazar que el proceso está bajo control/Proceso está bajo control)

Siguiendo la anterior expresión y teniendo en cuenta la nomenclatura del gráfico 5.4, si el proceso está bajo control, se tiene que:

$$
\begin{aligned}
\alpha_{\text {gráfico DS-U }} & =P\left(u_{i} \in \text { Región Rechazo Etapa } 1\right)+ \\
& +P\left(\left(u_{i} \in \text { R.Atención Etapa } 1\right) \cap\left(u_{i} \in \text { R.Rechazo Etapa } 2\right)\right)
\end{aligned}
$$




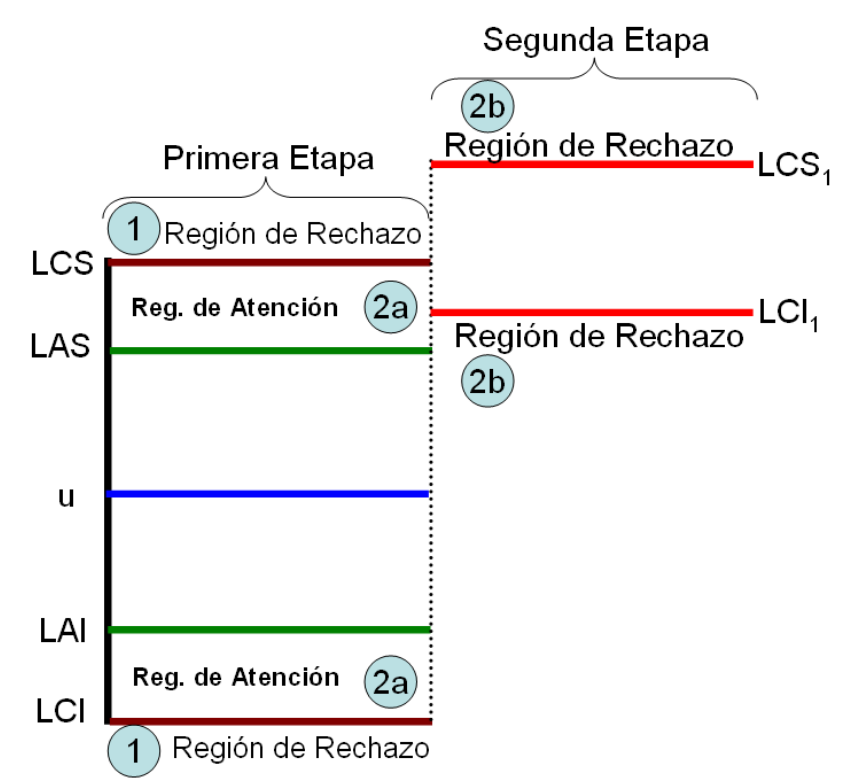

Fig. 5.4: Gráfico de control DS-U

o bien:

$$
\alpha_{\text {gráfico DS-U }}=P(\langle 1\rangle)+P(\langle 2 a\rangle \cap\langle 2 b\rangle)
$$

Entonces, la fórmula del error de tipo I es:

$$
\begin{aligned}
\alpha_{\text {gráfico DS-U }}= & \left(1-\sum_{i=0}^{f l o o r(L C S)} \frac{e^{-n_{1} \cdot p_{0} \cdot\left(n_{1} \cdot p_{0}\right)^{i}}}{i !}\right)+\left(\sum_{i=0}^{f l o r(L C I)} \frac{e^{-n_{1} \cdot p_{0} \cdot\left(n_{1} \cdot p_{0}\right)^{i}}}{i !}\right)+ \\
& +\left(\left(\sum_{i=\operatorname{cil} l(L A S)}^{f l o o r(L C S)} \frac{e^{-n_{1} \cdot p_{0} \cdot\left(n_{1} \cdot p_{0}\right)^{i}}}{i !}\right) \cdot\left(1-\sum_{j=c i l l(L C I 1)}^{f l o o r(L C S 1)} \frac{e^{-n_{2} \cdot p_{0} \cdot\left(n_{2} \cdot p_{0}\right)^{j}}}{j !}\right)\right)+ \\
& +\left(\left(\sum_{i=\operatorname{ceill}(L C I)}^{f l o o r(L A I)} \frac{e^{-n_{1} \cdot p_{0} \cdot\left(n_{1} \cdot p_{0}\right)^{i}}}{i !}\right) \cdot\left(1-\sum_{j=\operatorname{ceil}(L C I 1)}^{f l o o r(L C S 1)} \frac{e^{-n_{2} \cdot p_{0} \cdot\left(n_{2} \cdot p_{0}\right)^{j}}}{j !}\right)\right)
\end{aligned}
$$

y de forma análoga, la potencia del gráfico $D S-U$ es: 


$$
\begin{aligned}
& P O T_{\text {gráfico DS-U }}\left(p_{1}\right)=(1-\beta)_{\text {gráfico DS-U }}= \\
& =\left(1-\sum_{i=0}^{\text {floor }(L C S)} \frac{e^{-n_{1} \cdot p_{1} \cdot\left(n_{1} \cdot p_{1}\right)^{i}}}{i !}\right)+\left(\sum_{i=0}^{\text {floor }(L C I)} \frac{e^{-n_{1} \cdot p_{1} \cdot\left(n_{1} \cdot p_{1}\right)^{i}}}{i !}\right)+ \\
& +\left(\left(\sum_{i=\operatorname{ceil}(L A S)}^{\text {floor }(L C S)} \frac{e^{-n_{1} \cdot p_{1} \cdot\left(n_{1} \cdot p_{1}\right)^{i}}}{i !}\right) \cdot\left(1-\sum_{j=\operatorname{ceil}(L C I 1)}^{\text {floor }(L C S 1)} \frac{e^{-n_{2} \cdot p_{1} \cdot\left(n_{2} \cdot p_{1}\right)^{j}}}{j !}\right)\right)+ \\
& +\left(\left(\sum_{i=\operatorname{ceil}(L C I)}^{\operatorname{lloor}(L A I)} \frac{e^{-n_{1} \cdot p_{1} \cdot\left(n_{1} \cdot p_{1}\right)^{i}}}{i !} \cdot\left(1-\sum_{j=\operatorname{ceil}(L C I 1)}^{\operatorname{floor}(L C S I)} \frac{e^{-n_{2} \cdot p_{1} \cdot\left(n_{2} \cdot p_{1}\right)^{j}}}{j !}\right)\right)\right.
\end{aligned}
$$

Por último, en procesos bajo control, el tamaño medio de muestra del gráfico $D S-U$ es:

$$
\begin{gathered}
E[n]=n_{1}+n_{2} P(\text { Pasar a la Etapa 2) } \\
E[n]_{\text {gráfico DS-U }}=n_{1}+n_{2} \quad \cdot\left(\sum_{i=c e i l(L A S)}^{\text {floor }(L C S)} \frac{e^{-n_{1} \cdot p_{0} \cdot\left(n_{1} \cdot p_{0}\right)^{i}}}{i !}+\right. \\
\left.+\sum_{i=c e i l(L C I)}^{\text {floor }(L A I)} \frac{e^{-n_{1} \cdot p_{0} \cdot\left(n_{1} \cdot p_{0}\right)^{i}}}{i !}\right)
\end{gathered}
$$

Para desarrollar el trabajo propuesto para esta Tesis Doctoral, se compara

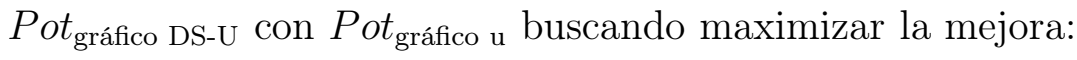

\section{$M A X\left[\right.$ Pot $_{\text {gráfico DS-U }}-$ Pot $\left._{\text {gráfico u }}\right]$}

Si Pot $_{\text {gráfico DS-U }}-$ Pot $_{\text {gráfico u }}>0$ indica, en ese caso, un mejor comportamiento del gráfico $u$ modificado.

- Sobre la notación matemática empleada a partir de este punto, los subíndices: gráfico DS-U o gráfico u, se indicarán indistintamente con los subíndices en forma abreviada: $D S-U$ o $u$, respectivamente. Por ejemplo:

$E[n]_{\text {gráfico DS-U }}=E[n]_{D S-U}$ 



\section{Capítulo 6}

\section{Comparación de la potencia del gráfico $u$ frente a la potencia del gráfico $D S-U$ en un valor $u_{1}$}

\subsection{Introducción.}

El objetivo principal de este capítulo es la comparación de la potencia del gráfico $u$ frente al valor de la potencia en el gráfico $D S-U$ en un valor $u_{1}$.

Para conocer mejor las propiedades del gráfico $D S-U$ se va a dividir el análisis de la potencia en dos zonas:

- Zona o tramo inferior $\Rightarrow$ Estudio de la potencia en $u_{1}<u_{0}$

- Zona o tramo superior $\Rightarrow$ Estudio de la potencia en $u_{1}>u_{0}$

Los resultados mostrados en este capítulo se han obtenido mediante un software programado para este problema concreto, y que hace uso de los 
Algoritmos Genéticos. La explicación detallada de este software y de los parámetros implicados en el mismo se encuentra en el capítulo 4.

En las siguientes secciones se va a analizar los resultados obtenidos por las simulaciones realizadas con la aplicación informática, en la que se ha seleccionado la opción de Mejora en un valor $u_{1}$.

En la tabla 6.1 se detallan los distintos casos objeto de análisis, que se ha utilizado para extraer conclusiones acerca del comportamiento del nuevo gráfico $D S-U$ planteado, frente al gráfico u clásico.

\begin{tabular}{||c|c|c||}
\hline \hline$\alpha_{\text {teórico }}$ & $n$ & \multicolumn{1}{|c||}{$\alpha_{\text {real }}$} \\
\hline \hline \multicolumn{2}{||l|}{$u_{0}=1$} \\
\hline 0.01 & 8 & 0.008566 \\
0.05 & 8 & 0.047935 \\
0.0027 & 8 & 0.003718021 \\
\hline 0.01 & 40 & 0.009132 \\
0.05 & 40 & 0.047396 \\
0.0027 & 40 & 0.003631613 \\
\hline \hline$u_{0}=5$ & \\
\hline 0.01 & 8 & 0.009131637 \\
0.05 & 8 & 0.047396 \\
0.0027 & 8 & 0.003631613 \\
\hline 0.01 & 40 & 0.009857263 \\
0.05 & 40 & 0.051681 \\
0.0027 & 40 & 0.00269305 \\
\hline \hline
\end{tabular}

Tabla 6.1: Descripción de los casos estudiados

Para un estudio más exhaustivo, en el anexo A se muestran todas las tablas y los gráficos de curvas de potencia del gráfico $D S-U$ comparados con 
la curva de potencia del gráfico $u$.

En este capítulo se incluyen los gráficos de diferencia de potencia $\operatorname{Pot}_{D S-U}$ y $P_{0} t_{u}$ agrupados por casos en los que los parámetros de entrada son tamaño de muestra y valor de diseño $u_{0}$ iguales, comparando su evolución con respecto a tres valores de $\alpha$ distintos. También se hace una segunda comparación en función de tamaños de muestra distintos, pero idénticos valores de $\alpha$ y $u_{0}$.

Se define la distancia $d_{u_{1}}$, que será la que empleada para determinar en qué valores de $u_{1}$ se va a estudiar la tendencia de $\operatorname{Pot}_{D S-U}$ con respecto a $\operatorname{Pot}_{u}$. Se estudiarán los siguientes valores: $\left[u_{0}-9 \cdot d_{u_{1}}, u_{0}+10 \cdot d_{u_{1}}\right]$, en separaciones de $d_{u_{1}}$ unidades. Por tanto se ensayarán dichos valores de $u_{1}$ por cada caso, incluyendo $u_{0}$, considerándolos suficientes para analizar el comportamiento de la potencia en el gráfico propuesto $D S-U$ frente al gráfico clásico $u$, ya que normalmente se pretende ser capaz de detectar cambios a valores de $u_{1}$ próximos a $u_{0}$. 


\subsection{Resultados.}

En esta sección se analizan detalladamente los resultados obtenidos en las simulaciones realizadas en los casos propuestos y que se describen en la tabla 6.1.

Se ha empleado un doble código en las tablas resumen que se encuentran en cada subsección y al final del capítulo para describir qué sucede en el valor de la potencia y del tamaño de muestra, y que queda desglosado en la tabla 6.2 .

\begin{tabular}{|cl|ll|}
\hline Rojo & Empeora & $\downarrow$ & Disminuye \\
\hline Verde & Mejora & $\uparrow \quad$ Aumenta \\
\hline Gris & Iguala & $=$ & Iguala \\
\hline$\downarrow=$ & En algunos valores se mantiene y en otros disminuye \\
\hline$\uparrow=$ & En algunos valores se mantiene y en otros se incrementa \\
\hline$\downarrow \uparrow$ & Algunos aumentan y otros disminuyen \\
\hline
\end{tabular}

Tabla 6.2: Leyenda de las tablas resumen de resultados obtenidos

\subsubsection{Comparación de las curvas de potencia cuando el valor de diseño del gráfico $\boldsymbol{u}$ clásico es $u_{0}=1$.}

Para la comparación de las curvas de potencia cuando el valor de diseño del gráfico $u$ clásico es $u_{0}=1$, se ha considerado que la distancia entre los valores estudiados sea: $d_{u_{1}}=0.1$, con lo que se obtienen valores para la comparación de las potencias de los gráficos $u$ y $D S-U$ en los siguientes $u_{1}$ además del propio $u_{0}=1$ : 


\begin{tabular}{|l|l|l|l|l|l|l|l|l|l|l|}
\hline$u_{1}$ & 0.1 & 0.2 & 0.3 & 0.4 & 0.5 & 0.6 & 0.7 & 0.8 & 0.9 & 1.0 \\
\hline \multirow{6}{*}{1.1} & 1.2 & 1.3 & 1.4 & 1.5 & 1.6 & 1.7 & 1.8 & 1.9 & 2.0 \\
\cline { 2 - 9 }
\end{tabular}

Para cada uno de los valores de $u_{1}$ se ha calculado los límites de control del gráfico $D S-U$, que se pueden consultar en el anexo A. Los gráficos de la figura 6.5 representan la diferencia entre la potencia del nuevo gráfico $D S$ $U$ y el gráfico clásico $u\left(\operatorname{Pot}_{D S-U}-\right.$ Pot $\left._{u}\right)$ para cada uno de los valores $u_{1}$ ensayados cuando $u_{0}=1$ y $n=8$; y $u_{0}=1$ y $n=40$, respectivamente. En esta figura se ha representado gráficamente y de forma conjunta para todos los $u_{1}$ analizados la mejora obtenida en cada $u_{1}$. Los gráficos correspondientes a la mejora de potencia en cada $u_{1}$ particular se encuentran en el anexo A.

Podemos concluir:

- En general, se observa que en las dos figuras no existe ningún valor negativo de la diferencia de potencias

$$
\left[\operatorname{Pot}_{D S-U}-\operatorname{Pot}_{u}\right]>0
$$

con lo que siempre se consigue una mejora de la potencia cuando $u_{0}=1$, independientemente del tamaño de muestra $n$.

- El comportamiento de $\left[\operatorname{Pot}_{D S-U}-P o t_{u}\right]$ es distinto en función del tamaño de muestra $n$, aunque como se ha apuntado, siempre es positiva.

- Cuando $n=8$, la mejora de la potencia va aumentando conforme $u_{1}$ se aleja de $u_{0}$, aunque en los valores más extremos de $u_{1}$ disminuye un poco dicha mejora. La mejora de potencia abarca la totalidad de los $u_{1}$ experimentados.

- Por otro lado, cuando se aumenta el tamaño de muestra hasta $n=40$, la mejora de potencia se concentra en los valores más inmediatamente próximos a $u_{0}$, hasta llegar a una $\operatorname{Pot}_{D S-U}-$ Pot $_{u}$ 
máxima o valor de pico. A partir de dicho valor máximo de pico, a mayor distancia de $u_{0}$ disminuye hasta no existir diferencia de potencias con respecto al gráfico $u$ en los valores más alejados de $u_{0}$ estudiados, es decir, ayuda a mejorar en desviaciones pequeñas y medias

\begin{tabular}{|l|c|c|c|c|}
\cline { 2 - 5 } & \multicolumn{4}{|c|}{ Valor máximo de pico de } \\
\cline { 2 - 5 } Pot $_{D S-U}-$ Pot $_{u}$ \\
\cline { 2 - 5 } & \multicolumn{2}{|c|}{$n=8$} & \multicolumn{2}{c|}{$n=40$} \\
\cline { 2 - 5 } & $\begin{array}{c}\text { Tramo } \\
\text { Inferior }\end{array}$ & $\begin{array}{c}\text { Tramo } \\
\text { Superior }\end{array}$ & $\begin{array}{c}\text { Tramo } \\
\text { Inferior }\end{array}$ & $\begin{array}{c}\text { Tramo } \\
\text { Superior }\end{array}$ \\
\hline \hline$\alpha=0.0027$ & 0.977 & 0.218 & 0.176 & 0.389 \\
\hline$\alpha=0.01$ & 0.747 & 0.173 & 0.352 & 0.294 \\
\hline$\alpha=0.05$ & 0.297 & 0.107 & 0.160 & 0.241 \\
\hline
\end{tabular}

Tabla 6.3: Valores máximos de pico de $\operatorname{Pot}_{D S-U}-\operatorname{Pot}_{u}$ para $u_{0}=1$

Estos gráficos también se pueden analizar dividiéndolos en dos tramos: $u_{1}<u_{0}$ o Tramo Inferior y $u_{1}>u_{0}$ o Tramo Superior:

- Tramo Inferior.

- En el caso de $n=8$, en la gráfica 6.1a, se muestra que conforme nos alejamos de $u_{0}=1$, la diferencia de potencia se incrementa a excepción de los valores más extremos ensayados, y esta diferencia es mayor conforme $\alpha$ disminuye.

- En la gráfica 6.1 b se muestra que, cuando $n=40$, la mejora no es tan marcada como en el caso anterior $(n=8)$, pero detecta bien cambios a valores más cercanos del valor de diseño $u_{0}$. Los valores de pico de $\left[\operatorname{Pot}_{D S-U}-P_{o t}\right]$ se muestran en la tabla 6.3. 
- Tramo Superior

- Cuando $n=8$, la mejora es moderada, más si se compara con el tramo inferior, y sigue el mismo patrón: a menor $\alpha$, mayor es el incremento de potencia respecto al gráfico $u$. En la tabla 6.3 se puede hacer la comparación de los valores de pico de ambos tramos, siendo los correspondientes al tramo inferior mucho mayores que los del tramo superior.

- Para el caso en que se aumenta a $n=40$, el comportamiento de la diferencia de las potencias es similar al caso de $n=8$ cuando se varía $\alpha$, pero en este caso las ganancias en potencia son mayores, incluso superan en la mayoría de $u_{1}$ los incrementos en el tramo inferior, a excepción del caso en que $\alpha=0.01$, en el que la curva de $\left[\operatorname{Pot}_{D S-U}-P o t_{u}\right]$ es prácticamente simétrica respecto a $u_{0}$.

Hasta ahora se ha visto que se puede mejorar la potencia en un valor $u_{1}$, sin embargo se necesita estudiar qué ocurre en cada caso con el comportamiento de la potencia del resto de valores cuando se está mejorando la potencia en un valor $u_{1}$.

Cuando se maximiza $\left[\operatorname{Pot}_{D S-U}-\right.$ Pot $\left._{u}\right]$ en valores de $u_{1}<u_{0}$ (tramo inferior), se aumenta la potencia de detección en este tramo inferior, pero a cambio, al mismo tiempo se empeora la potencia de detección en el tramo superior, siendo $\left[\operatorname{Pot}_{D S-U}-\operatorname{Pot}_{u}\right]<0$.

En cambio, si se maximiza $\left[\operatorname{Pot}_{D S-U}-P_{0} t_{u}\right]$ en valores de $u_{1}>u_{0} \mathrm{o}$ tramo superior, se obtiene que $\left[\operatorname{Pot}_{D S-U}-P_{o t}\right]>0$ en los casos de que $\alpha=0.0027$ y $\alpha=0.01$, contrariamente al apartado anterior, ahora se mejora la detección de variaciones de $u_{0}$ tanto en el tramo superior como en el inferior simultáneamente. Para cuando se tiene un valor de $\alpha=0.05$, no se puede garantizar la mejora en todos los valores de $u_{1}$, ya que en algunos mejora y en otros empeora. 
Las comparaciones de curvas de potencia de los gráficos $D S-U$ y u se encuentran en el anexo A y sirven para observar el comportamiento de la potencia del resto de valores cuando se mejora la potencia en un determinado valor $u_{1}$. Se han extraído dos gráficos del anexo A y se muestran en la figura 6.2 a modo de ejemplo.

En cuanto al tamaño medio de muestra, resaltar que se reduce el tamaño medio de muestra obtenido con el gráfico $D S-U$ con respecto al tamaño del gráfico de control $u$ inicial. En el caso de $n=40$ se observa una reducción del tamaño medio, que puede llegar a ser de 20 unidades por debajo del valor de referencia.

Cuando $n=8$ y el proceso está bajo control se muestrea una media de 6.70 unidades, y cuando se descentra el proceso sigue habiendo una disminución de $E[n]_{\text {gráfico DS-U. }}$

Cuando $n=40$, la diferencia del tamaño medio de muestra es mucho mayor incluso que en el caso anterior $(n=8)$. Cuando el proceso está bajo control, el ahorro de muestras a tomar es de hasta 10 unidades, y en algunos valores, cuando el proceso está fuera de control se llega hasta una reducción de 20 unidades de muestreo. 


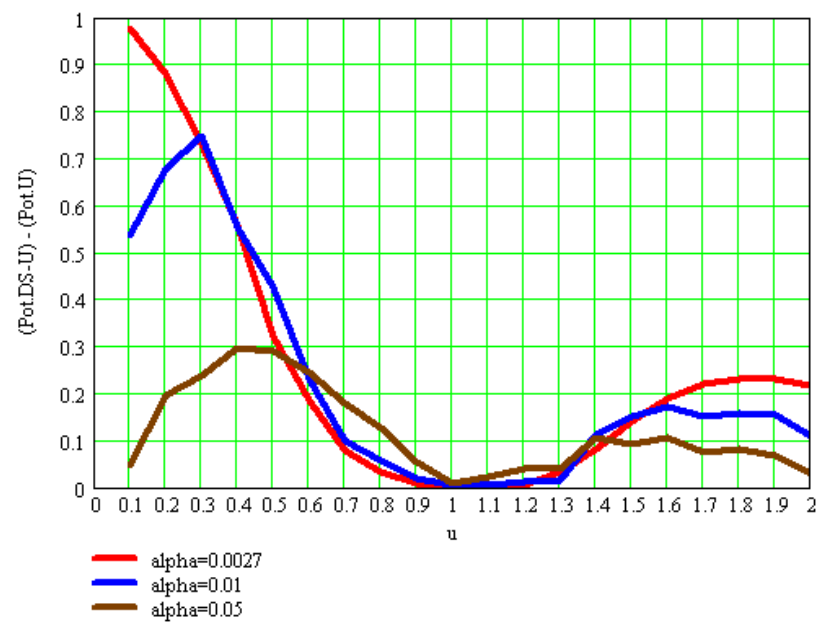

(a) $u_{0}=1$ y $n=8$

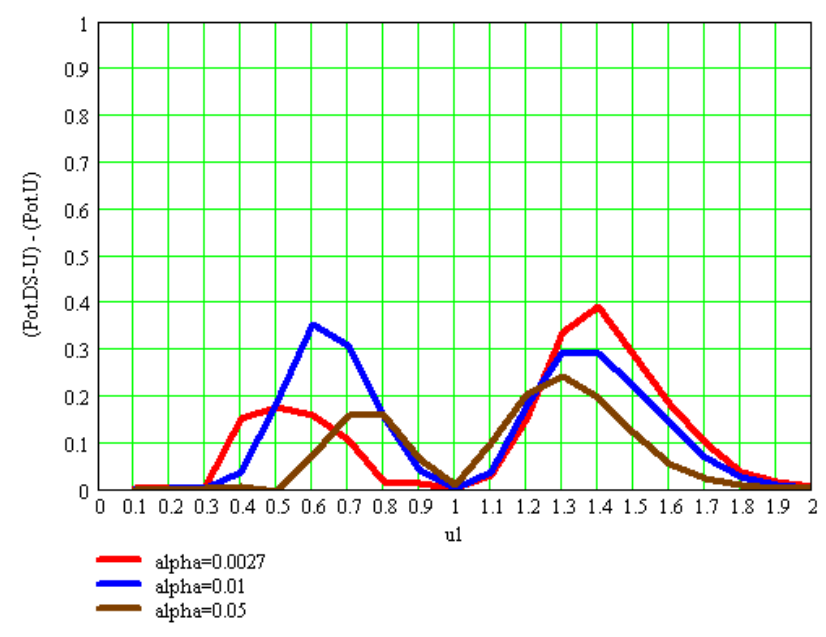

(b) $u_{0}=1$ y $n=40$

Fig. 6.1: Pot $_{D S-U}-$ Pot $_{u}$ 


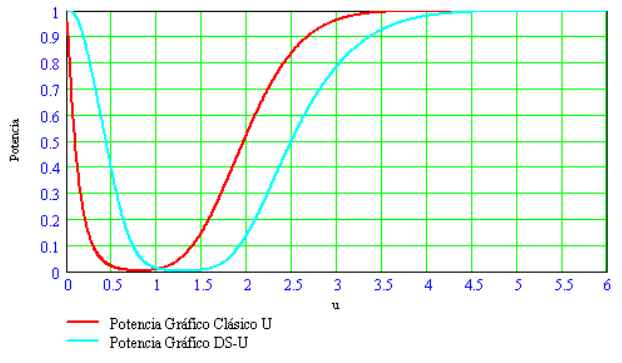

(a) $u_{1}=0.8$

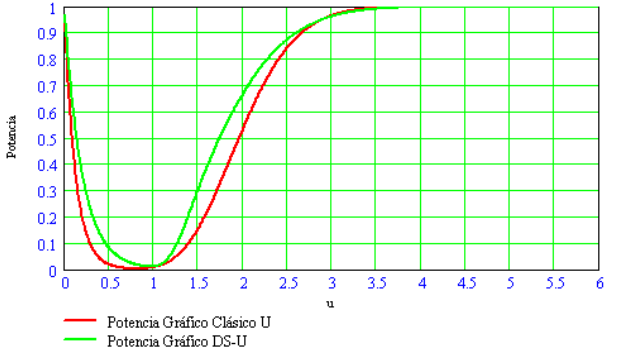

(b) $u_{1}=1.6$

Fig. 6.2: Comparación de curvas de potencia para $u_{0}=1, n=8$ y $\alpha=0.01$

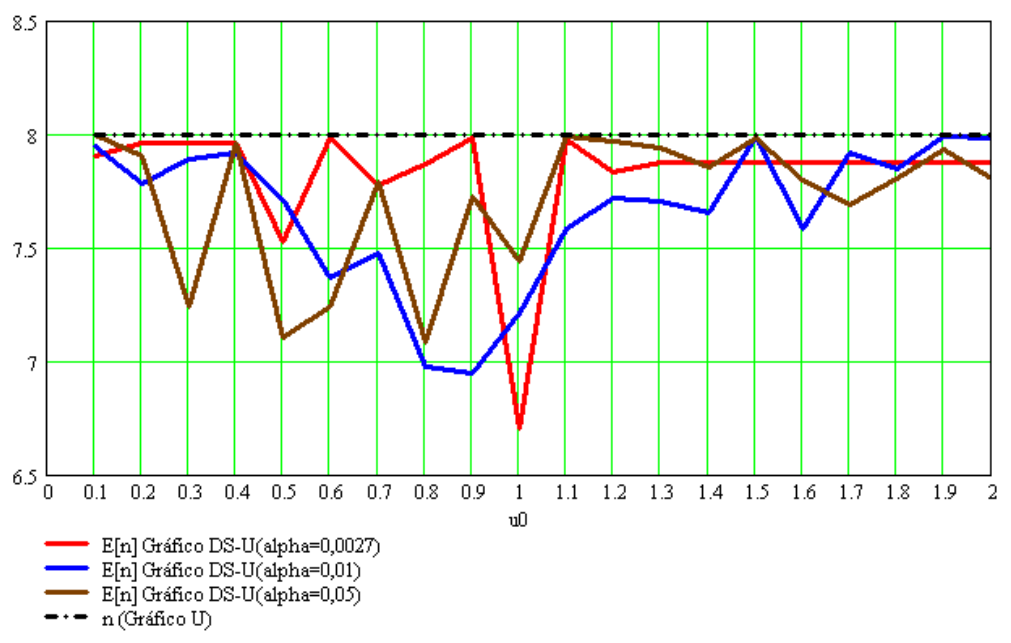

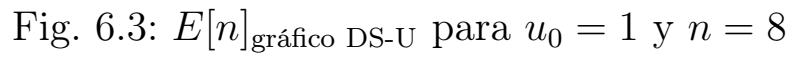




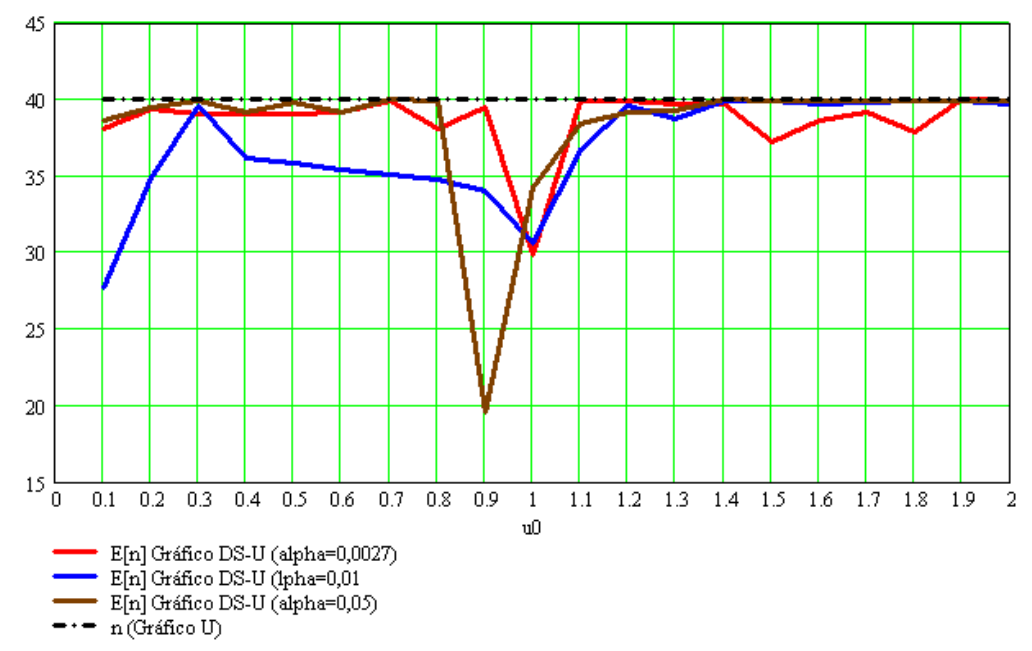

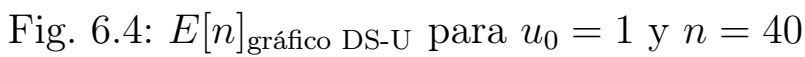

Para tener un compendio de la información aportada por los análisis realizados, se ha considerado interesante realizar una tablas que muestren, a modo de resumen, el comportamiento de la potencia en todos los casos que se pueden presentar según sea el valor de $u_{1}$ estudiado.

Se muestra en primer lugar una tabla-leyenda (tabla 6.4) para una mejor comprensión de las tablas de resumen posteriores. En ella se explica qué significa cada una de las casillas.

En la primera fila se clasifica la información según el tramo al que pertenezca el valor $u_{1}$ que se está ensayando: Tramo Inferior $\left(u_{1}<u_{0}\right)$ o Tramo $\operatorname{Superior}\left(u_{1}>u_{0}\right)$.

La primera columna consta de cuatro filas. En las tres primeras se indica qué sucede con la potencia en dicho valor $u_{1}$, o bien en el resto de valores de $u_{1}$ que pertenezcan al Tramo Inferior, o bien qué sucede en el resto de valores de $u_{1}$ que pertenezcan al Tramo Superior. En la última fila se pregunta qué pasa con el valor medio del tamaño de muestra $E[n]$ del gráfico $D S$ - $U$ con 
respecto al tamaño de muestra $n$ del gráfico $u$ clásico.

\begin{tabular}{|l|l|l|}
\cline { 2 - 3 } \multicolumn{1}{c|}{} & $\begin{array}{c}\text { Cuando } u_{1} \text { está } \\
\text { en el tramo } \\
\text { inferior }\left(u_{1}<u_{0}\right)\end{array}$ & $\begin{array}{c}\text { Cuando } u_{1} \text { está } \\
\text { en el tramo } \\
\text { superior }\left(u_{1}>u_{0}\right)\end{array}$ \\
\hline $\begin{array}{l}\text { Comportamiento de la potencia } \\
\text { en } u_{1} \text { (valor de referencia) }\end{array}$ & & \\
\hline $\begin{array}{l}\text { Comportamiento de la } \\
\text { potencia en otros } u_{1}<u_{0}\end{array}$ & & \\
\hline $\begin{array}{l}\text { Comportamiento de la } \\
\text { potencia en otros } u_{1}>u_{0}\end{array}$ & & \\
\hline Tamaño de Muestra & & \\
\hline
\end{tabular}

Tabla 6.4: Leyenda de las tablas resumen

En las tablas 6.5 y 6.6 se muestra para distintos valores de $\alpha$ qué sucede con la potencia para cada uno de los casos mencionados en la tabla 6.4.

Cuando $u_{0}=1$ y $n=8$ se observa en la tabla 6.5 que en la mayoría de los casos se mejora la potencia, excepto cuando trata de mejorar en un valor $u_{1}$ que es inferior a $u_{0}$. En dicho caso no se consigue mejorar en el tramo superior cuando sí se mejora en el tramo inferior.

Al incrementar el tamaño de muestra, cuando $u_{0}=1$ y $n=40$, las mejoras de potencia ya no son tan generalizadas como en el caso anterior. Se muestra en la tabla 6.6 un resumen de lo que sucede en cada caso, poniendo de relieve que existen muchas casillas de color rojo, lo que implica que en dicha circunstancia se empeora la actuación del gráfico $u$.

Aquí cabe mencionar que el programa diseñado no contempla la posibilidad de llegar a un gráfico clásico u como solución, así se intenta estudiar el comportamiento del gráfico propuesto $D S-U$ tanto en mejoras como en 


\begin{tabular}{|c|c|c|c|c|c|c|}
\hline \multicolumn{8}{|c|}{$u_{0}=1 \mathrm{y} n=8$} \\
\hline \hline \multirow{3}{*}{ ¿Qué pasa con } & \multicolumn{2}{|c|}{$\alpha=0.0027$} & \multicolumn{2}{|c|}{$\alpha=0.01$} & \multicolumn{2}{c|}{$\alpha=0.05$} \\
\cline { 2 - 8 } Pot $_{D S-U}$ en? $\downarrow$ & $u_{1} \in$ & $u_{1} \in$ & $u_{1} \in$ & $u_{1} \in$ & $u_{1} \in$ & $u_{1} \in$ \\
\hline$u_{1}$ & $\uparrow .1,0.9]$ & {$[1.1,2.0]$} & {$[0.1,0.9]$} & {$[1.1,2.0]$} & {$[0.1,0.9]$} & {$[1.1,2.0]$} \\
\hline Otros $u_{1}<u_{0}$ & $\uparrow$ & $\uparrow$ & $\uparrow$ & $\uparrow$ & $\uparrow$ & $\uparrow$ \\
\hline Otros $u_{1}>u_{0}$ & $\downarrow$ & $\uparrow$ & $\uparrow$ & $\uparrow$ & $\uparrow$ & $\downarrow \uparrow$ \\
\hline \hline Tamaño Muestra & $\downarrow$ & $\downarrow$ & $\downarrow$ & $\uparrow$ & $\downarrow$ & $\downarrow \uparrow$ \\
\hline
\end{tabular}

Tabla 6.5: Comportamiento del gráfico $D S-U$ frente al gráfico u cuando $u_{0}=1$ y $n=8$

disminuciones de potencia respecto al gráfico $u$.

\subsubsection{Comparación de las curvas de potencia cuando el valor de diseño del gráfico $\boldsymbol{u}$ clásico es $u_{0}=5$.}

En esta subsección se va a comparar los resultados obtenidos para el caso en que $u_{0}=5$. La distancia entre los valores estudiados es: $d_{u_{1}}=0.5$, con lo que se obtienen valores para la comparación de las potencias de los gráficos u y $D S-U$ en los siguientes $u_{1}{ }^{1}$ :

\begin{tabular}{|l|l|l|l|l|l|l|l|l|l|l|l|}
\hline$u_{1}$ & 0.1 & 0.5 & 1.0 & 1.5 & 2.0 & 2.5 & 3.0 & 3.5 & 4.0 & 4.5 & 5.0 \\
\hline \multirow{4}{*}{5.5} & 6.0 & 6.5 & 7.0 & 7.5 & 8.0 & 8.5 & 9.0 & 9.5 & 10.0 & \multicolumn{1}{|c}{} \\
\cline { 2 - 10 } & &
\end{tabular}

Para cada uno de los valores de $u_{1}$ se han calculado los límites de control

\footnotetext{
${ }^{1}$ Se ha añadido el primer valor $u_{1}=0.1$, aunque dado $\left(d_{u_{1}}=0.5\right)$ no correspondería, se considera interesante ver el comportamiento en este valor extremo, estudiándose 10 valores de $\left(u_{1}<u_{0}\right)$ y 10 valores $\left(u_{1}>u_{0}\right)$, además del propio $u_{0}$
} 


\begin{tabular}{|c|c|c|c|c|c|c|}
\hline \multicolumn{8}{|c|}{$u_{0}=1 \mathrm{y} n=40$} \\
\hline \hline \multirow{2}{*}{ ¿Qué pasa con } & \multicolumn{2}{|c|}{$\alpha=0.0027$} & \multicolumn{2}{|c|}{$\alpha=0.01$} & \multicolumn{2}{c|}{$\alpha=0.05$} \\
\cline { 2 - 8 } Pot $_{D S-U}$ en? $\downarrow$ & $u_{1} \in$ & $u_{1} \in$ & $u_{1} \in$ & $u_{1} \in$ & $u_{1} \in$ & $u_{1} \in$ \\
\hline$u_{1}$ & $\uparrow 0.1,0.9]$ & {$[1.1,2.0]$} & {$[0.1,0.9]$} & {$[1.1,2.0]$} & {$[0.1,0.9]$} & {$[1.1,2.0]$} \\
\hline Otros $u_{1}<u_{0}$ & $\downarrow \uparrow$ & $\uparrow$ & $\uparrow$ & $\uparrow$ & $\uparrow$ & $\uparrow$ \\
\hline Otros $u_{1}>u_{0}$ & $\downarrow \uparrow$ & $\uparrow$ & $\uparrow$ & $\downarrow$ & $\downarrow \uparrow$ & $\downarrow$ \\
\hline \hline Tamaño Muestra & $\downarrow$ & $\downarrow$ & $\downarrow$ & $\uparrow$ & $\downarrow$ & $\uparrow$ \\
\hline
\end{tabular}

Tabla 6.6: Comportamiento del gráfico $D S-U$ frente al gráfico u cuando $u_{0}=1$ y $n=40$

del gráfico que se encuentran en el anexo A. Para estudiar el comportamiento de la potencia se han representado estos valores en las gráficas de las figuras 6.5 a y $6.5 \mathrm{~b}$, en las que se puede observar el valor de $P_{0} t_{D S-U}-P o t_{u}$ para dos tamaños de muestra distintos: $n=8$ y $n=40$ respectivamente. Si la potencia del gráfico $D S$ - $U$ es mejor que la del gráfico u en un determinado $u_{1}$, entonces esta diferencia será positiva.

Tras observar y analizar las figuras $6.5 \mathrm{a}$ y $6.5 \mathrm{~b}$, se tiene que ambos casos son muy distintos, por lo que su análisis debe ser por separado. En la figura 6.5a se encuentra un patrón similar al hallado en la subsección anterior (6.2.1), en la que hay una mejora en la detección de variación a valores de $u_{1}$ próximos a $u_{0}$, que disminuye a medida que se aleja de $u_{0}$. En cambio, en la figura $6.5 \mathrm{~b}$ no se encuentra una mejora tan notoria como en los casos anteriores, además de comportarse en el tramo inferior incluso peor que el gráfico clásico $u$ de referencia, con valores de $\left(\operatorname{Pot}_{D S-U}-P_{o t}\right)<0$. 


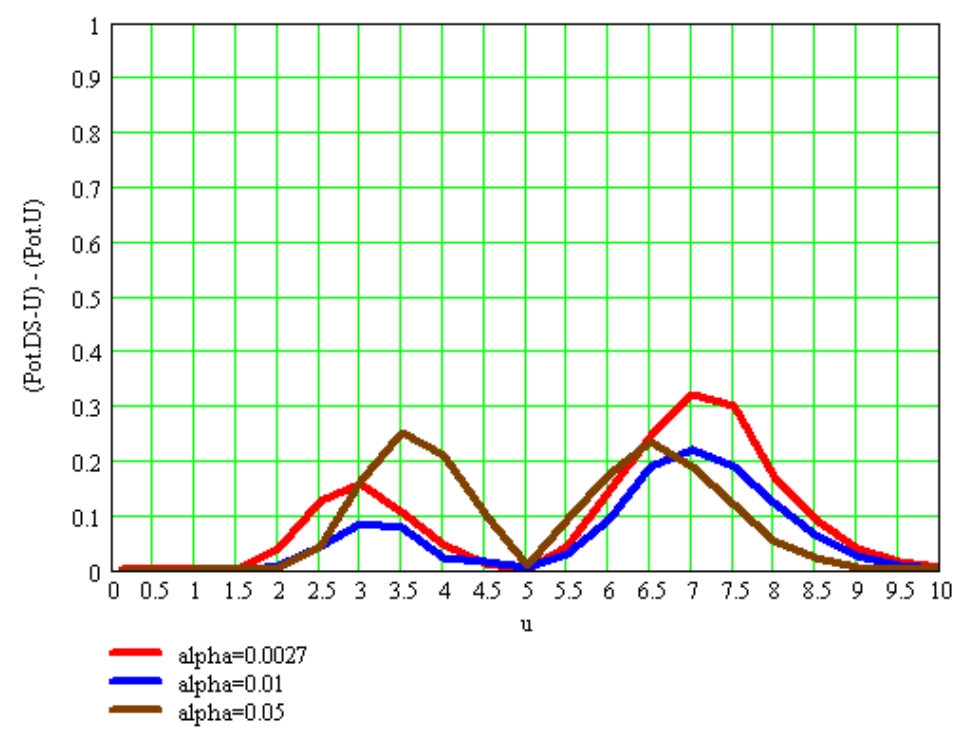

(a) $u_{0}=5$ y $n=8$

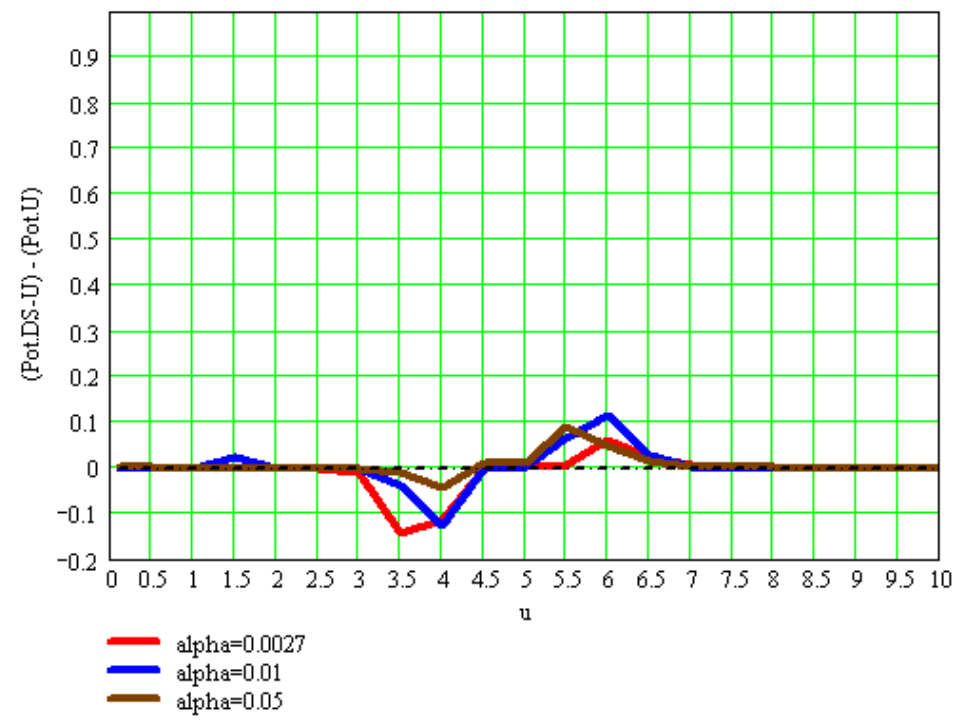

(b) $u_{0}=5$ y $n=40$

Fig. 6.5: Pot $_{D S-U}-$ Pot $_{u}$ 
Se puede obtener las siguientes conclusiones según el caso:

- $u_{0}=5$ y $n=8$ :

- En la gráfica 6.5 a se observa que a medida que $u_{1}$ se aleja de $u_{0}=5$, la diferencia de potencia se incrementa hasta llegar a un valor pico en $u_{1} \in[3.5,4]$ en el tramo inferior, y $u_{1} \in[6.5,7]$ en el tramo superior. A partir de estos máximos de $\operatorname{Pot}_{D S-U}-\operatorname{Pot}_{u}$, y alejándose de $u_{0}$, la diferencia de potencias disminuye hasta convertirse en nula.

- Cuando $\alpha=0.05$, la gráfica es casi simétrica respecto a $u_{0}=5$, consiguiéndose una $\operatorname{Pot}_{D S-U}-\operatorname{Pot}_{u}=0.252$, que es máxima a una distancia de $3 d_{u_{1}}$ de $u_{0}$.

- Cuando $\alpha=0.0027$ y $\alpha=0.01$, el patrón de comportamiento es muy parecido a lo que sucede en el caso anterior $(\alpha=0.05)$, pero esta vez no es simétrica respecto a $u_{0}$, sino que el valor de pico de $\operatorname{Pot}_{D S-U}-\operatorname{Pot}_{u}$ en el tramo inferior es algo menor que el correspondiente valor de pico del tramo superior, aunque en ambos casos están a la misma distancia de $u_{0}$. En la tabla 6.7 se observan dichos valores de pico en los tramos inferior y superior.

\begin{tabular}{|l|c|c|}
\cline { 2 - 3 } & \multicolumn{2}{c|}{$\begin{array}{c}\text { Valor máximo de pico de } \\
\text { Pot }_{D S-U}-\text { Pot }_{u}\end{array}$} \\
\cline { 2 - 3 } & Tramo Inferior & Tramo Superior \\
\hline \hline$\alpha=0.0027$ & 0.157 & 0.320 \\
\hline$\alpha=0.01$ & 0.085 & 0.220 \\
\hline$\alpha=0.05$ & 0.252 & 0.236 \\
\hline
\end{tabular}

Tabla 6.7: Valores máximos de pico de $\operatorname{Pot}_{D S-U}-P o t_{u}$ para $u_{0}=5$ y $n=8$ 


\begin{tabular}{|l|c|c|}
\cline { 2 - 3 } \multicolumn{1}{c|}{} & \multicolumn{2}{c|}{ Valor de pico de $\operatorname{Pot}_{D S-U}-$ Pot $_{u}$} \\
\cline { 2 - 3 } \multicolumn{1}{c|}{} & Mínimo del Tramo Inferior & Máximo del Tramo Superior \\
\hline$\alpha=0.0027$ & -0.145 & 0.062 \\
\hline$\alpha=0.01$ & -0.128 & 0.114 \\
\hline$\alpha=0.05$ & -0.042 & 0.089 \\
\hline
\end{tabular}

Tabla 6.8: Valores de pico de $\operatorname{Pot}_{D S-U}-\operatorname{Pot}_{u}$ para $u_{0}=5 \mathrm{y}$ $n=40$

- $u_{0}=5$ y $n=40$ : En este caso existe una diferencia significativa en el comportamiento de la potencia con respecto a tramos.

- En el tramo inferior no se mejora con respecto al gráfico clásico $u$, e incluso no consigue igualar la actuación de éste, sino que el gráfico $D S-U$ se comporta peor en valores de $u_{1}$ cercanos a $u_{0}$, como se ve en los valores mínimos reflejados en la tabla 6.8.

- En el tramo superior se consigue una mejora leve en valores hasta $3 \cdot d_{u_{1}}$, que inmediatamente disminuyen hasta no haber diferencia entre las potencias de los gráficos u y $D S-U$. En la tabla 6.8 se reflejan las diferencias máximas que se encuentran en el tramo superior.

Ahora se va a estudiar el comportamiento de la potencia en otros valores de $u$ cuando estamos intentando mejorar la potencia en un valor $u_{1}$ determinado.

Cuando se maximiza $\operatorname{Pot}_{D S-U}-\operatorname{Pot}_{u}$ en valores de $u_{1}<u_{0}$ (tramo inferior), se aumenta la potencia de detección en este tramo inferior, pero a cambio, al mismo tiempo se empeora la potencia de detección en el tramo superior, siendo $\operatorname{Pot}_{D S-U}-$ Pot $_{u}<0$. Lo mismo sucede a la inversa, si se maximiza $\operatorname{Pot}_{D S-U}-\mathrm{Pot}_{u}$ en valores de $u_{1}>u_{0}$ o tramo superior, se obtiene 
que $\operatorname{Pot}_{D S-U}-$ Pot $_{u}<0$ en todos los casos experimentados con diferentes valores de $\alpha$.

En el anexo A se pueden observar las gráficas que comparan la curva de potencia del gráfico $D S-U$ en cada $u_{1}$ con respecto a la curva de potencia del gráfico $u$, y más concretamente, a partir de la sección A.7 de dicho anexo, en la que se muestran los gráficos referentes a los casos ensayados con $u_{0}=5$.

En la figura 6.6 se muestran dos gráficos incluidos en el anexo A a modo de ejemplo, en el que se comprueba que mejorando en un tramo, se empeora el comportamiento en el tramo opuesto. 


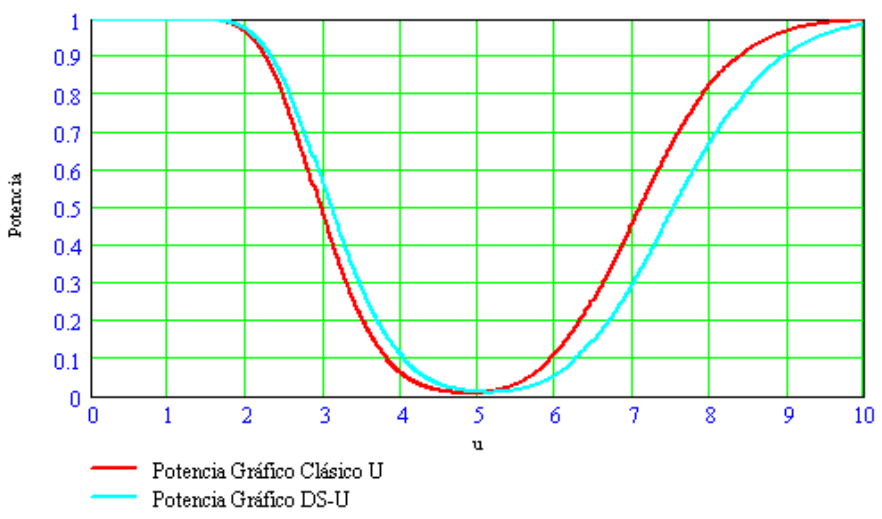

(a) $u_{1}=3.5$

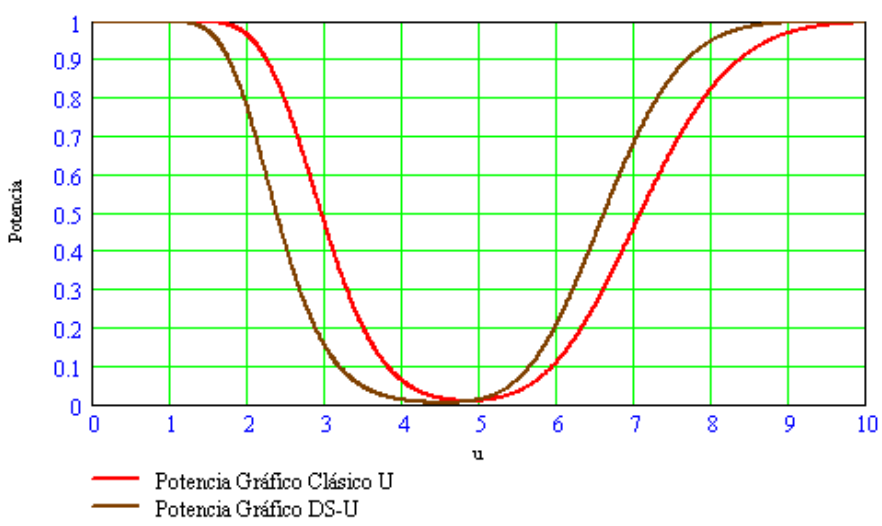

(b) $u_{1}=7.0$

Fig. 6.6: Curvas de potencia para $u_{0}=5, n=8$ y $\alpha=0.01$ 
Por lo que respecta al tamaño medio de muestra, en el gráfico 6.7 se ha representado el valor medio del tamaño de muestra del gráfico $D S-U$ para los distintos valores de $u_{1}$ analizados.

Para los casos ensayados en los que $n=8$ el tamaño medio de muestra se reduce en mayor medida en el Tramo Inferior. Cuando el proceso está bajo control se reduce hasta dos unidades con respecto al tamaño de muestra del gráfico clásico $u$ : $E[n]=6$ cuando $\alpha=0,01$.

En cambio, para $n=40$ se observa una fuerte reducción del valor medio de tamaño de muestra en el valor de referencia $u_{1}=u_{0}$, en el que se llega a incluso hasta 32 unidades menos, lo cual es notorio y a tener en cuenta. En las figuras 6.7 y 6.8 se ven las gráficas correspondientes al tamaño de muestra en el gráfico $D S-U$, y en ellas se puede ver la variación del mismo que hay para diferentes $u_{1}$.

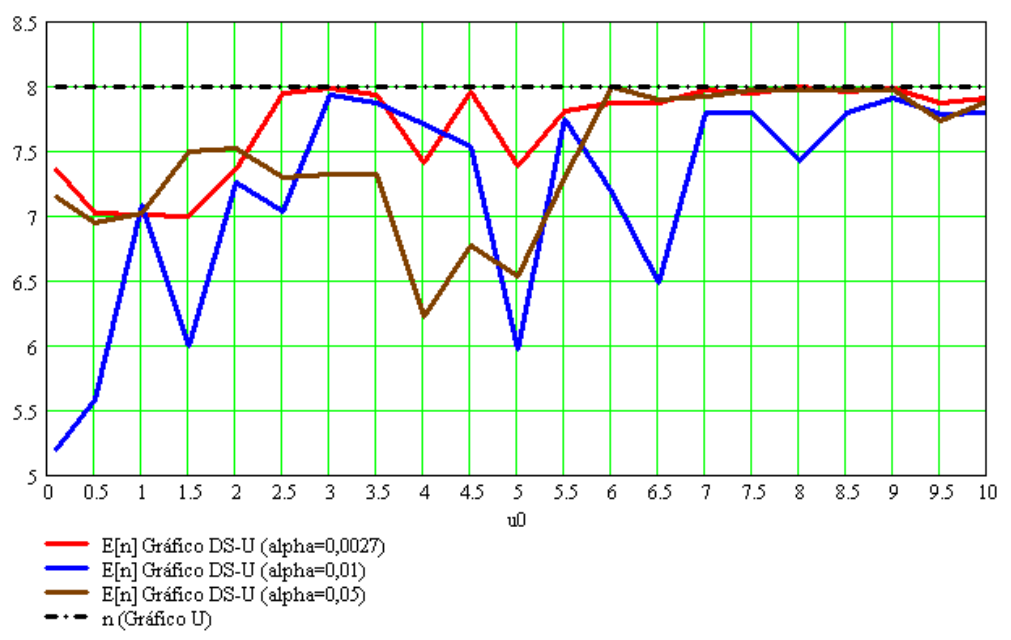

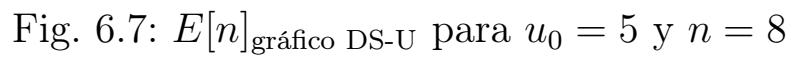

Como en la subsección anterior, se tiene que analizar todos los parámetros que afectan al comportamiento de la potencia en el nuevo gráfico $D S-U$ con 


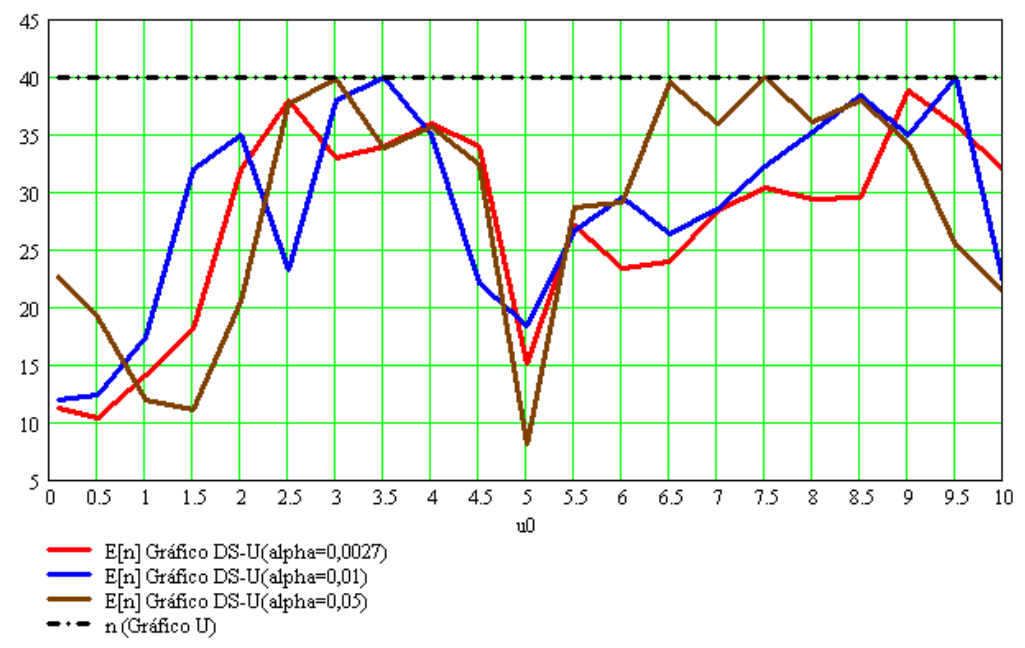

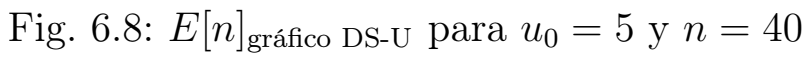

respecto al gráfico $u$. Entre otros factores, el valor de $\alpha$ es mínimamente distinto al valor de partida. En ese caso habría que sopesar, según sea el proceso en el que se va a aplicar un gráfico de control, la importancia de reducir las unidades de muestreo, mejorando la potencia de detección con el nuevo gráfico $D S$ - $U$. Estos valores de $\alpha$ están en los anexos correspondientes a cada caso (ver Anexo A).

En las tablas 6.9 y 6.10 se muestra el resumen del comportamiento de la potencia y el tamaño de muestra en cada uno de los casos siguiendo la leyenda explicada en la tabla 6.2. 


\begin{tabular}{|c|c|c|c|c|c|c|}
\hline \multicolumn{7}{|c|}{$u_{0}=5$ y $n=8$} \\
\hline \multirow[b]{2}{*}{$\begin{array}{l}\text { ¿Qué pasa con } \\
\operatorname{Pot}_{D S-U} \text { en? } \Downarrow\end{array}$} & \multicolumn{2}{|c|}{$\alpha=0.0027$} & \multicolumn{2}{|c|}{$\alpha=0.01$} & \multicolumn{2}{|c|}{$\alpha=0.05$} \\
\hline & $\begin{array}{c}u_{1} \in \\
{[0.1,4.5]}\end{array}$ & $\begin{array}{c}u_{1} \in \\
{[5.5,10.0]}\end{array}$ & $\begin{array}{c}u_{1} \in \\
{[0.1,4.5]}\end{array}$ & $\begin{array}{c}u_{1} \in \\
{[5.5,10.0]}\end{array}$ & $\begin{array}{c}u_{1} \in \\
{[0.1,4.5]}\end{array}$ & $\begin{array}{c}u_{1} \in \\
{[5.5,10.0]}\end{array}$ \\
\hline$u_{1}$ & $\uparrow$ & $\uparrow$ & $\uparrow$ & $\uparrow$ & $\uparrow$ & $\uparrow$ \\
\hline Otros $u_{1}<u_{0}$ & $\uparrow$ & $\downarrow$ & $\downarrow \uparrow$ & $\downarrow$ & $\uparrow$ & $\downarrow$ \\
\hline Otros $u_{1}>u_{0}$ & $\downarrow \uparrow$ & $\uparrow$ & $\downarrow \uparrow$ & $\uparrow$ & $\downarrow$ & $\uparrow$ \\
\hline Tamaño Muestra & $\downarrow$ & $\downarrow=$ & $\downarrow$ & $\downarrow$ & $\downarrow$ & $\downarrow=$ \\
\hline
\end{tabular}

Tabla 6.9: Comportamiento del gráfico $D S-U$ frente al gráfico $u$ cuando $u_{0}=5$ y $n=8$

\begin{tabular}{|c|c|c|c|c|c|c|}
\hline \multicolumn{7}{|c|}{$u_{0}=5$ y $n=40$} \\
\hline \multirow[b]{2}{*}{$\begin{array}{l}\text { ¿Qué pasa con } \\
\operatorname{Pot}_{D S-U} \text { en? } \Downarrow\end{array}$} & \multicolumn{2}{|c|}{$\alpha=0.0027$} & \multicolumn{2}{|c|}{$\alpha=0.01$} & \multicolumn{2}{|c|}{$\alpha=0.05$} \\
\hline & $\begin{array}{c}u_{1} \in \\
{[0.1,4.5]}\end{array}$ & $\begin{array}{c}u_{1} \in \\
{[5.5,10.0]}\end{array}$ & $\begin{array}{c}u_{1} \in \\
{[0.1,4.5]}\end{array}$ & $\begin{array}{c}u_{1} \in \\
{[5.5,10.0]}\end{array}$ & $\begin{array}{c}u_{1} \in \\
{[0.1,4.5]}\end{array}$ & $\begin{array}{c}u_{1} \in \\
{[5.5,10.0]}\end{array}$ \\
\hline$u_{1}$ & $\downarrow$ & $\downarrow=$ & $\downarrow=$ & $\uparrow=$ & $\downarrow=$ & $\uparrow=$ \\
\hline Otros $u_{1}<u_{0}$ & $\downarrow$ & $\downarrow$ & $\downarrow$ & $\downarrow$ & $\downarrow \uparrow$ & $\downarrow$ \\
\hline Otros $u_{1}>u_{0}$ & $\downarrow$ & $\uparrow=$ & $\downarrow$ & $\downarrow \uparrow$ & $\downarrow$ & $\uparrow$ \\
\hline Tamaño Muestra & $\downarrow$ & $\downarrow$ & $\downarrow=$ & $\downarrow=$ & $\downarrow=$ & $\downarrow=$ \\
\hline
\end{tabular}

Tabla 6.10: Comportamiento del gráfico $D S-U$ frente al gráfico $u$ cuando $u_{0}=5$ y $n=40$ 


\subsubsection{Comparación de las curvas de potencia en función del tamaño de muestra $n$, fijando $u_{0} \mathbf{y} \alpha$.}

En las subsecciones anteriores se hace la comparación de curvas de potencia para valores de $n$ y $u_{0}$ prefijados. Si ahora se hace la comparación de dichas curvas de potencia desde otro punto de vista, en el que se fijan los valores de $\alpha$ y $u_{0}$ y se estudia la evolución según el tamaño de muestra $n$, obtendremos un análisis más exhaustivo acerca del comportamiento de la potencia en este caso.

En la figura 6.9 se muestra una gráfica comparativa resumen de las curvas de $\operatorname{Pot}_{D S-U}-\operatorname{Pot}_{u}$ en el caso particular de que $u_{0}=1$, y para cada $u_{1}$. La comparación de las curvas clasificadas según el valor de $\alpha$ - las mismas que en la figura 6.9, pero separadas por parejas con el mismo valor de $\alpha^{-}$, se encuentran en el anexo A.13.

Cuando $u_{0}=1$, podemos separar el análisis de la figura 6.9 en dos, según sea el tamaño de muestra $n$ :

- $n=8 \Rightarrow$ En todo el rango de valores ensayados se mejora la potencia, aunque en los valores de $u_{1}$ más próximos a $u_{0}$ son mejoras inferiores en el Tramo Superior que en el siguiente caso, cuando $n=40$.

- $n=40 \Rightarrow$ La mejora de la potencia se concentra en los valores de $u_{1}$ más próximos a $u_{0}$, y en el caso del Tramo Superior la mejora es mucho mayor que cuando $n=8$. 
CAPÍTULO 6. COMPARACIÓN DE LA POTENCIA DEL GRÁFICO U FRENTE A LA POTENCIA DEL GRÁFICO DS - U EN UN VALOR U

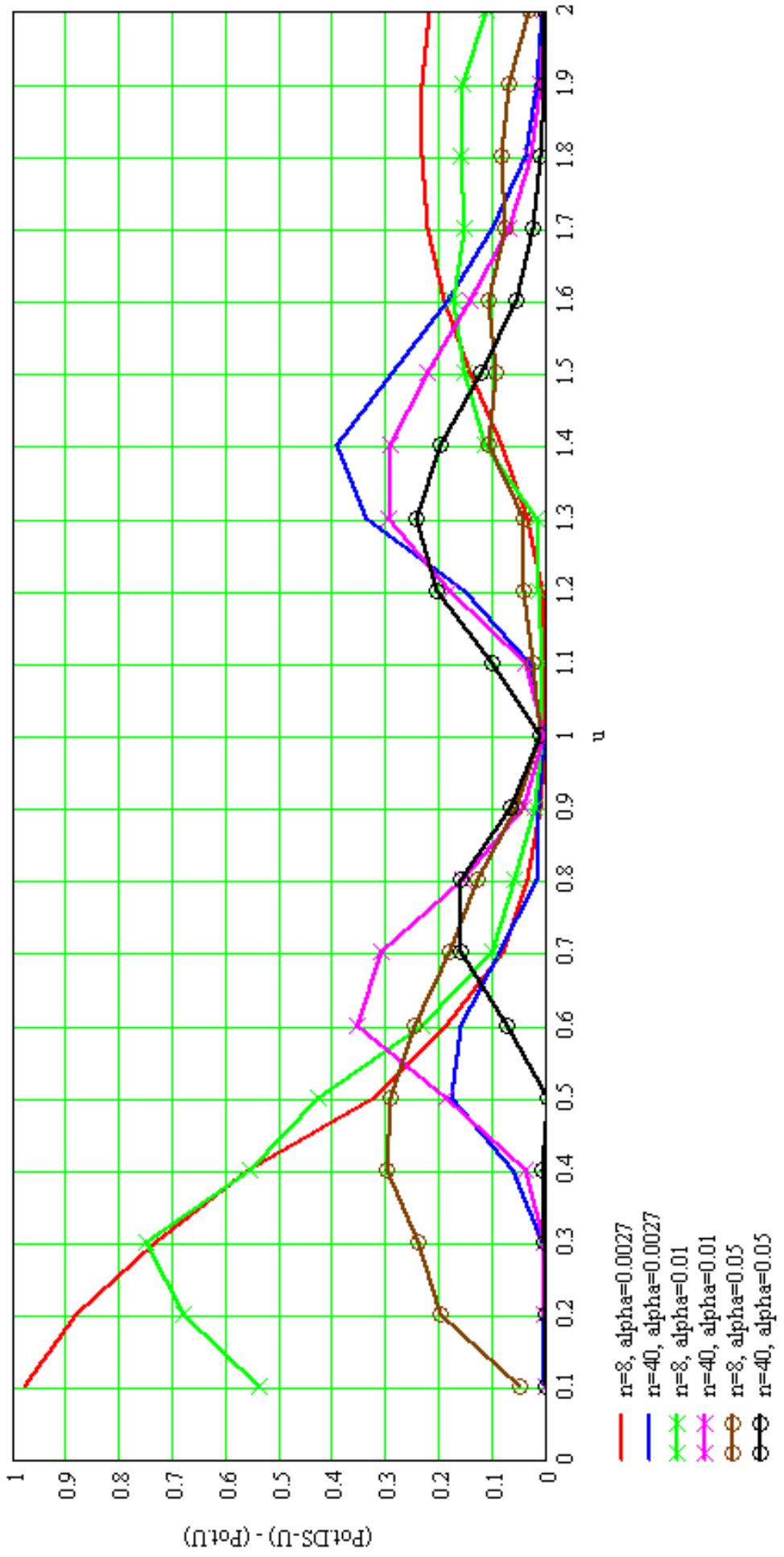

83

Fig. 6.9: Pot $_{D S-U}-$ Pot $_{u}$ para $u_{0}=1$ 
CAPÍTULO 6. COMPARACIÓN DE LA POTENCIA DEL GRÁFICO U FRENTE A LA POTENCIA DEL GRÁFICO DS - U EN UN VALOR U

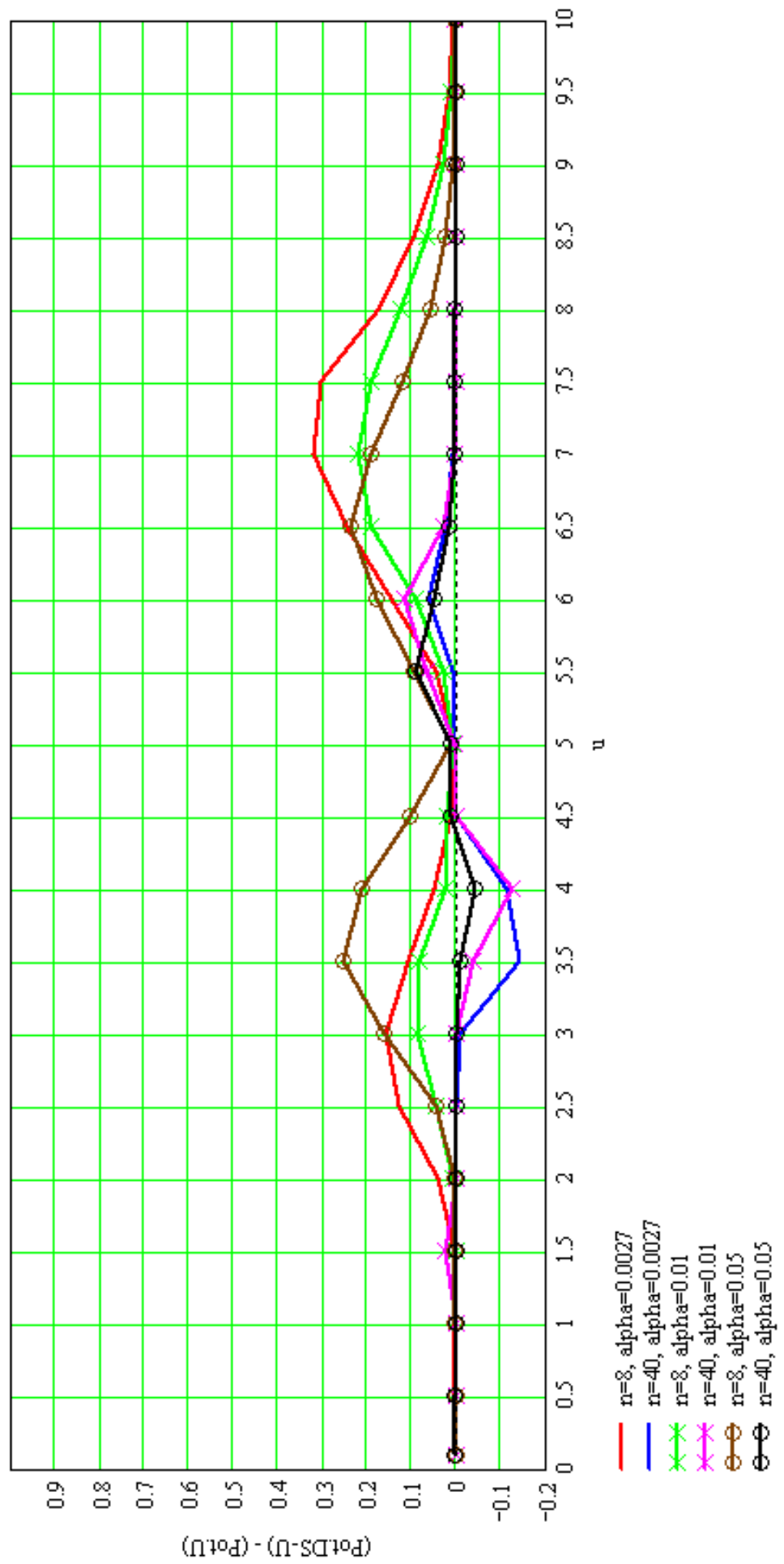

84

Fig. 6.10: $\operatorname{Pot}_{D S-U}-$ Pot $_{u}$ para $u_{0}=5$ 
Por lo que respecta al caso en que $u_{0}=5$, también se puede separar el análisis de la figura 6.10 en función del tamaño de muestra $n$ :

- $n=8 \Rightarrow$ Las mejoras de potencia están concentradas en valores próximos a $u_{0}$, al contrario que cuando $u_{0}=1$, en el que se producen mejoras en todos los valores de $u_{1}$ estudiados.

- $n=40 \Rightarrow$ La potencia del gráfico $D S-U$ empeora o como mucho iguala la potencia del gráfico $D S-U$ en el Tramo Inferior. En lo referente al Tramo Superior, las mejoras de potencia del nuevo gráfico están muy concentradas en los valores de $u_{1}$ próximos a $u_{0}$, y además, considerablemente menores que en el caso de $n=8$.

\subsection{Conclusiones}

A continuación se exponen las principales conclusiones:

- Se consigue mejorar en $u_{1}$ para todos $\operatorname{los} \alpha$, a excepción del caso particular en que $u_{1}=5$ y $n=40$, donde disminuye la potencia porque disminuye considerablemente el tamaño medio de muestra.

- El gráfico $D S-U$ presenta interesantes ventajas para tamaños de muestra pequeños, y también para valores de $u_{0}$ pequeños.

- El valor de $\alpha$ influye en cuánto mejora el gráfico. 


\subsection{Ejemplo}

Tras las conclusiones obtenidas en este capítulo, se va a proponer un ejemplo de aplicación a un proceso concreto:

En una fábrica dedicada a la inyección de plásticos se tiene un proceso de fabricación en una máquina inyectora en el que se considera un número de defectos por unidad muestreada de $u_{0}=1.3 \mathrm{y}$ no debe sobrepasar $u_{1}=1.5$.

Actualmente la empresa está tomando un tamaño de muestra $n=10 \mathrm{y}$ se ha fijado un $\alpha=0.01$. Se está usando un gráfico clásico con las siguientes características: $L C S=2.2284$ y $L C I=0.3715$.

En la imagen 6.11 se puede ver detalles de la máquina inyectora objeto de este ejemplo.

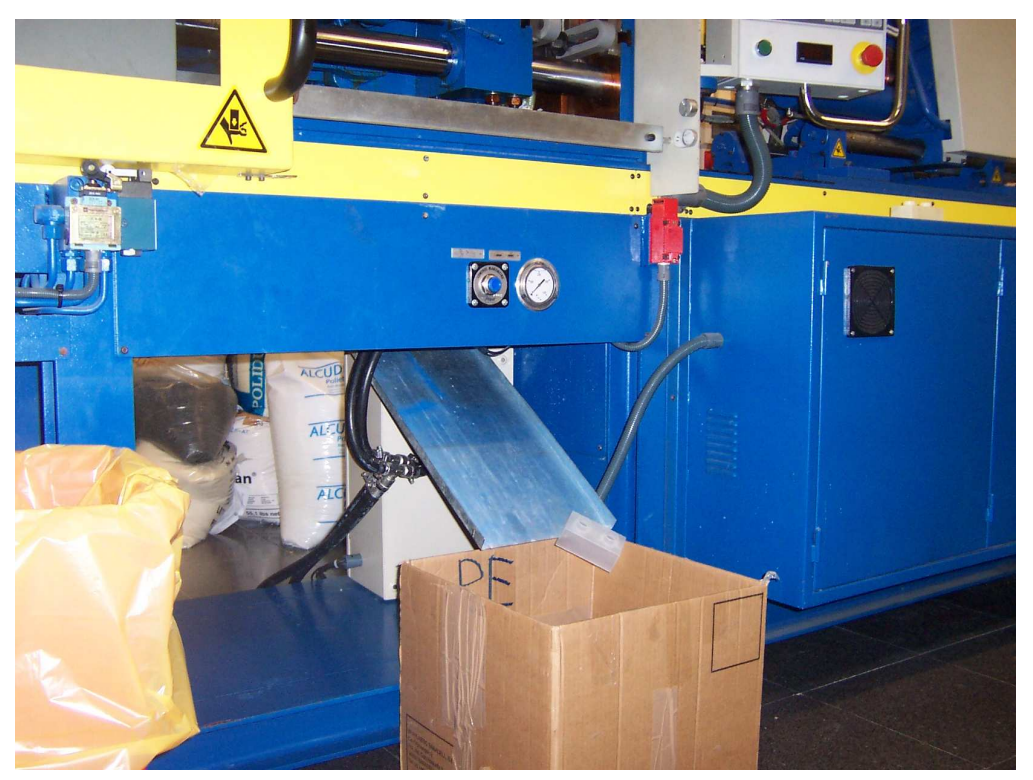

Fig. 6.11: Pieza saliendo de la máquina inyectora 
Ahora se introducen los parámetros en el software programado para esta Tesis Doctoral para intentar mejorar la potencia de detección en $u_{1}=1.5$.

Los límites calculados para el nuevo gráfico $D S-U$ son los representados en la figura 6.12.

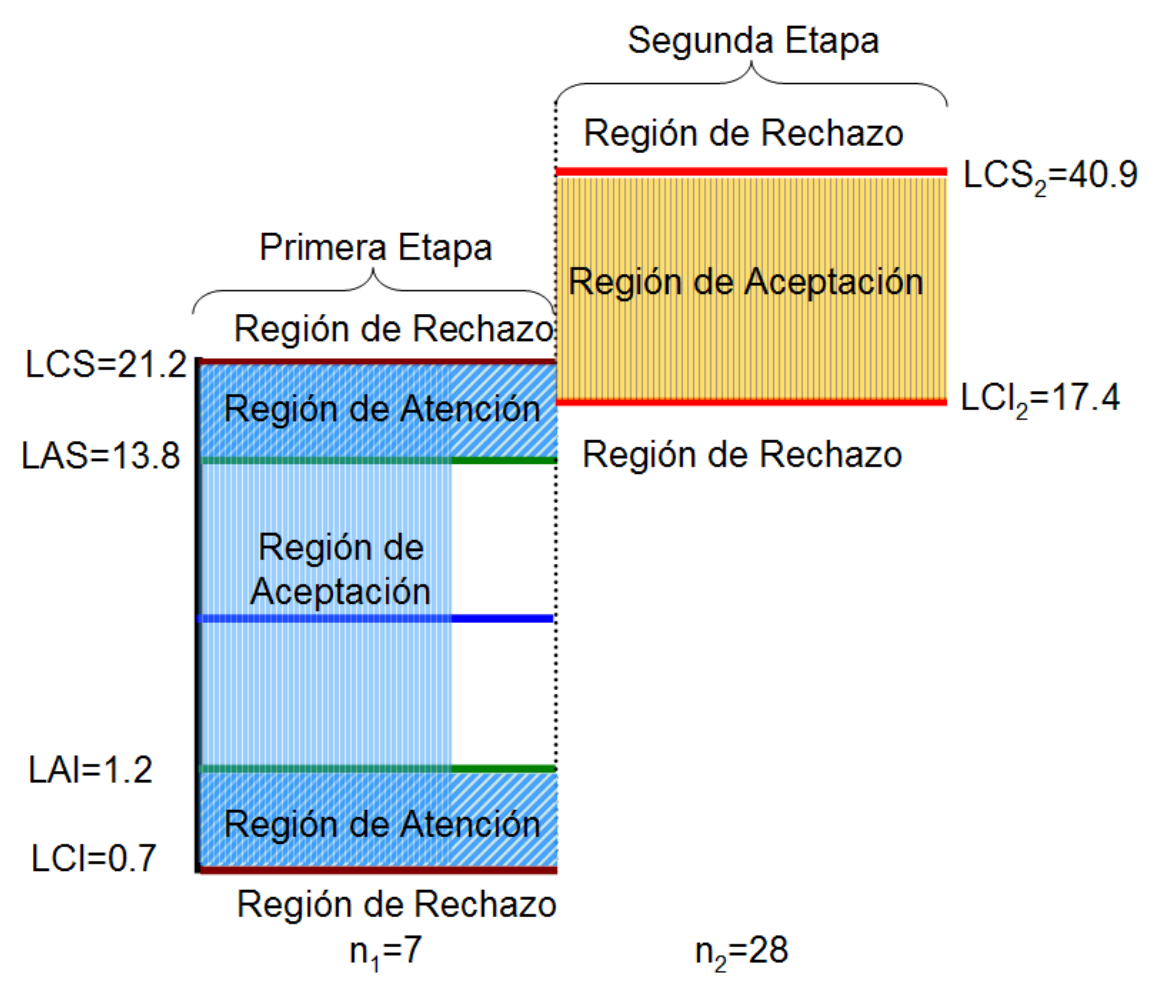

Fig. 6.12: Gráfico de Control DS-U

La potencia del gráfico $D S-U$ en $u_{1}$ es 0.102 , frente a la potencia del gráfico $u$, que en ese mismo $u_{1}$ que vale 0.053 . En este caso particular la potencia mejora en 0.049, es decir, se consigue doblar la potencia del gráfico $u$. También se puede analizar en términos de $A R L$, este $A R L$ pasa de 18.9 unidades a 9.8 unidades, se reduce a la mitad. 
En la gráfica 6.13 se representa la curva de la diferencia de potencias entre el gráfico $D S-U$ y el gráfico $u$ : $\operatorname{Pot}_{D S-U}-P_{o t}$. En ella se puede observar que la diferencia de potencias es negativa en valores de $u_{1}<u_{0}$, y a partir de $u_{0}$ toma valores positivos, lo que indica que este gráfico diseñado para este ejemplo, mejora en el Tramo Superior, pero a su vez, empeora los resultados para el Tramo Inferior. Este resultado se corresponde con las conclusiones extraídas en este capítulo para un caso en el $n$ y $u_{0}$ sean valores pequeños.

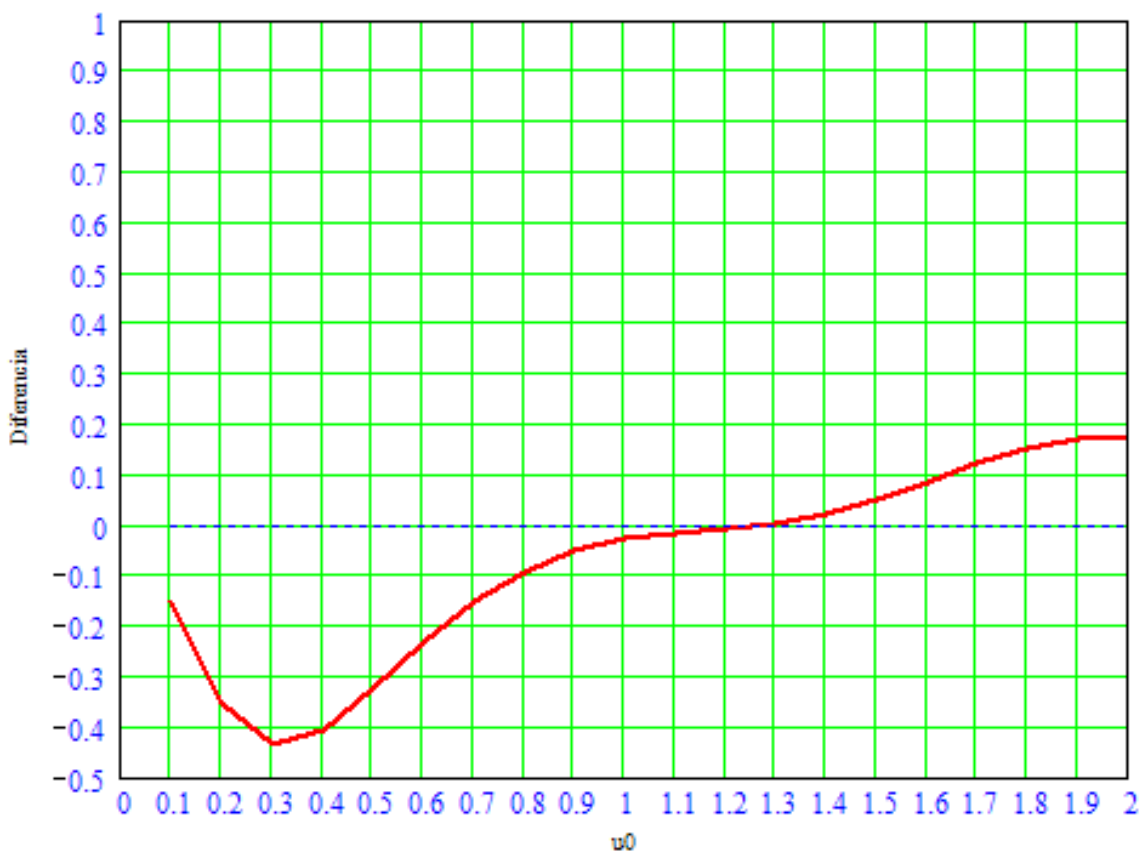

Fig. 6.13: Pot $_{D S-U}-$ Pot $_{u}$ para $n=10, u_{0}=1.3$ y $\alpha_{t}=0.01$

En la figura 6.14 se muestran las dos curvas de potencia, representada de color rojo la curva de potencia del gráfico $u$ y de color azul la curva de la potencia del gráfico $D S-U$. 


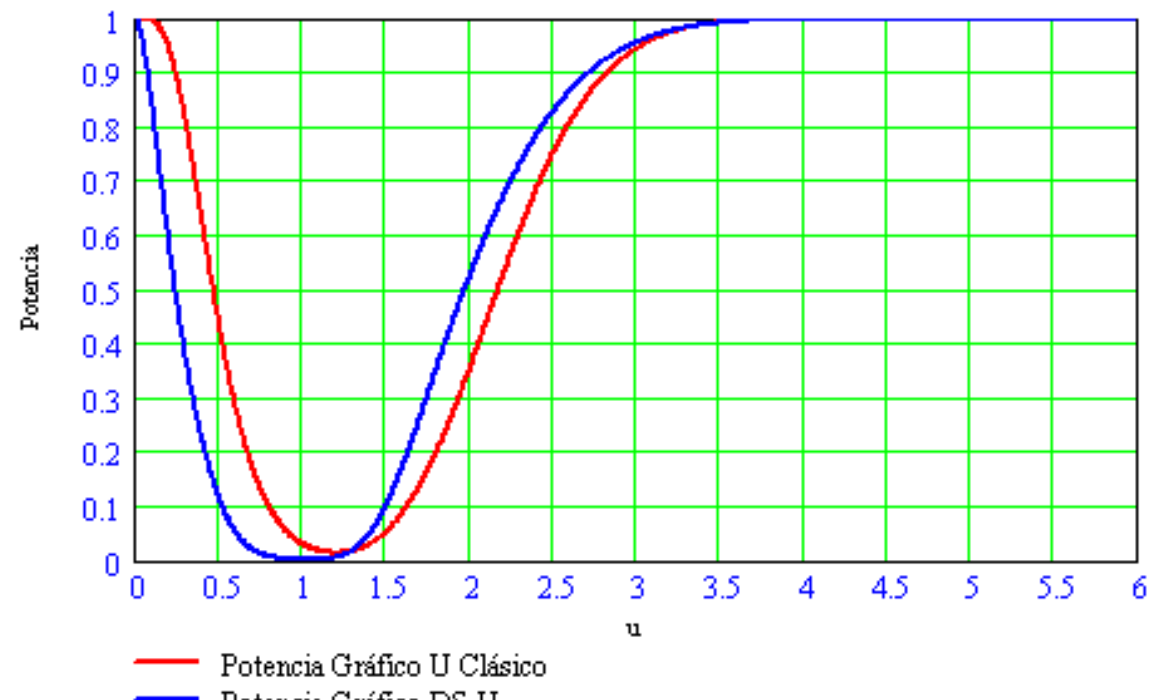

Potencia Gráfico DS-U

Fig. 6.14: Curvas de potencia para $u_{1}=1,5$ 


\section{Capítulo 7}

\section{Comparación de la potencia del gráfico $u$ frente a la potencia del gráfico $D S-U$ en}

$u_{1} \in\left[u_{\text {inferior }}, u_{\text {superior }}\right]$

\subsection{Introducción.}

En este capítulo se va a tratar de mejorar la función de potencia de un gráfico de control $u$ en un intervalo $\left[u_{\text {inferior }}, u_{\text {superior }}\right]$, a partir de ahora denotados de la siguiente forma: $\left[u_{i}, u_{s}\right]$.

Se va a emplear la aplicación informática desarrollada en el capítulo 6, con las mismas posibilidades de modificación de parámetros, seleccionando ahora la opción MEJORA EN UN INTERVALO.

La representación gráfica de la curva de potencia del gráfico u clásico se 
compara con la curva de potencia del nuevo gráfico planteado, de forma que se maximice la diferencia entre ambas curvas.

Por tanto, lo que se busca es:

$$
M A X\left[\sum_{u=u_{\mathrm{i}}}^{u_{\mathrm{s}}} \operatorname{Pot}_{\text {gráfico DS-U }}(u)-\operatorname{Pot}_{\text {gráfico u }}(u)\right]
$$

\subsection{Metodología empleada.}

Al estudiar la tendencia de la potencia se ha seleccionado en todos los casos cinco valores por encima del valor de diseño y otros cinco valores por debajo, para así analizar mejor la posibilidad de detección de los cambios a valores próximos al valor de diseño.

El algoritmo seleccionará la solución que proporcione una mayor suma de diferencias de potencias en todos los $u_{1}$ dentro de dicho intervalo. Si $\left[\operatorname{Pot}_{D S-U}-\operatorname{Pot}_{u}\right]<0$ en un valor $u_{1} \in\left[u_{i}, u_{s}\right]$, se considerará como una penalización a la suma total de diferencias de potencias. Por tanto, habrá soluciones que sean muy buenas en el sentido de que puede que en varios valores $u_{1}$ la diferencia de potencia positivas sume más que en otras soluciones, pero basta con que tenga unos cuantos valores negativos lo suficientemente bajos como para que deje de ser una buena solución.

Por ejemplo, si el valor de diseño es $u_{0}=1$, el programa acumulará el valor de $\left[\operatorname{Pot}_{D S-U}-P_{o t}\right]$ en los valores $u_{1} \in[0.5,1.5]$, separados por $d_{u_{1}}=0,1$ unidades. En este caso en particular, se estudiaría la suma de $\left[\right.$ Pot $_{D S-U}-$ Pot $\left._{u}\right]$ en los siguientes valores:

\begin{tabular}{|l|l|l|l|l|l|l|l|l|l|l|l|}
\hline$\left[u_{i}, u_{s}\right]:$ & 0.5 & 0.6 & 0.7 & 0.8 & 0.9 & 1.0 & 1.1 & 1.2 & 1.3 & 1.4 & 1.5 \\
\hline
\end{tabular}


Puede que en algunos casos no se mejore la potencia en todos los puntos, pero la suma de las diferencias sí es la mejor que ha encontrado.

Como se ha visto en el capítulo anterior, es difícil conseguir un conjunto de parámetros del gráfico $D S-U$ que consigan mejorar la potencia en todos los valores $u_{1}$ al mismo tiempo.

En la tabla 7.1 se muestran los distintos casos objeto de análisis en este capítulo, y a partir de los cuales se extraerán conclusiones.

\begin{tabular}{|c||c|c|}
\hline \hline$u_{0}$ & $\alpha_{\text {teórico }}$ & $n_{\text {gráfico u }}$ \\
\hline \hline 1 & 0.01 & 8 \\
1 & 0.05 & 8 \\
\hline \hline 1 & 0.01 & 40 \\
1 & 0.05 & 40 \\
1 & 0.0027 & 40 \\
\hline \hline 5 & 0.01 & 8 \\
5 & 0.05 & 8 \\
5 & 0.0027 & 8 \\
\hline \hline
\end{tabular}

Tabla 7.1: Descripción de los casos estudiados.

A continuación se van a mostrar los resultados obtenidos por grupos, para los casos en que se tenga el mismo valor de $n$ así como también un valor de $u_{0}$ coincidente, pero distinto valor de $\alpha$. Aquí se mostrarán algunas gráficas y datos, pero toda la información detallada se encuentra en el Anexo B. 


\subsection{Resultados}

\subsubsection{Comparación de la potencia de los gráficos de control u y $\boldsymbol{D S}-\boldsymbol{U}$ con $n=8$ y $u_{0}=1$}

El primer ejemplo que se va a estudiar tiene como parámetros del gráfico clásico $u: n=8$ y $u_{0}=1$, y se realizará la comparativa para $\alpha=0.01 \mathrm{y}$ $\alpha=0.05$.

En la tabla 7.2 quedan reflejados los resultados que se han obtenido para los casos estudiados en que $n=8$ con dos variantes: $\alpha=0.01$ y $\alpha=0.05$. En ninguno de los dos casos se ha conseguido optimizar en todos y cada uno de los puntos del intervalo, aunque se puede observar en la gráfica 7.1, que en el caso de que $\alpha$ sea menor, la mejora es mayor, y además se consigue optimizar en todo el tramo inferior al valor de diseño $u_{0}=1$.

\begin{tabular}{|c|c|c|c|c|c|c|c|c|}
\hline \multicolumn{3}{|c|}{$u_{0}=1$} & \multicolumn{3}{|c|}{$\alpha_{\text {teórico }}=0.01$} & \multicolumn{3}{|c|}{$n=8$} \\
\hline$\alpha_{t}$ & $L C I$ & $L A I$ & $L C I_{1}$ & $L A S$ & $L C S$ & $L C S_{1}$ & $n_{1}$ & $n_{2}$ \\
\hline \multirow[t]{2}{*}{0.01} & 1.1 & 1.7 & 82.9 & 14.4 & 18.5 & 100.5 & 7 & 100 \\
\hline & \multicolumn{3}{|c|}{$\alpha_{u}=8.566474 \cdot 10^{-3}$} & \multicolumn{3}{|c|}{$\alpha_{D S-U}=0.010276$} & \multicolumn{2}{|c|}{$E[n]_{D S-U}=7.56$} \\
\hline \multicolumn{3}{|c|}{$u_{0}=1$} & \multicolumn{3}{|c|}{$\alpha_{\text {teórico }}=0.05$} & \multicolumn{3}{|c|}{$n=8$} \\
\hline$\alpha_{t}$ & $L C I$ & $L A I$ & $L C I_{1}$ & $L A S$ & $L C S$ & $L C S_{1}$ & $n_{1}$ & $n_{2}$ \\
\hline 0.05 & 1.1 & 1.6 & 77.9 & 9.4 & 15.7 & 92.8 & 5 & 92 \\
\hline & \multicolumn{3}{|c|}{$\alpha_{u}=0.047935$} & \multicolumn{3}{|c|}{$\alpha_{D S-U}=0.057473$} & \multicolumn{2}{|c|}{$E[n]_{D S-U}=7.92$} \\
\hline
\end{tabular}

Tabla 7.2: Solución obtenida para gráficos con $n=8$ y $u_{0}=1$

En cuanto al tamaño medio de muestra, en ambos casos es superior a $n=7$, con lo que la reducción del tamaño de muestra no es considerable 
con respecto al gráfico clásico $u$. Además, en los dos ejemplos se observa que el valor de $\alpha_{D S-U}$ es ligeramente superior a $\alpha_{u}$, con lo que se tendría que admitir este pequeño incremento del error de primera especie para mejorar en algún tramo de valores la potencia del nuevo gráfico.

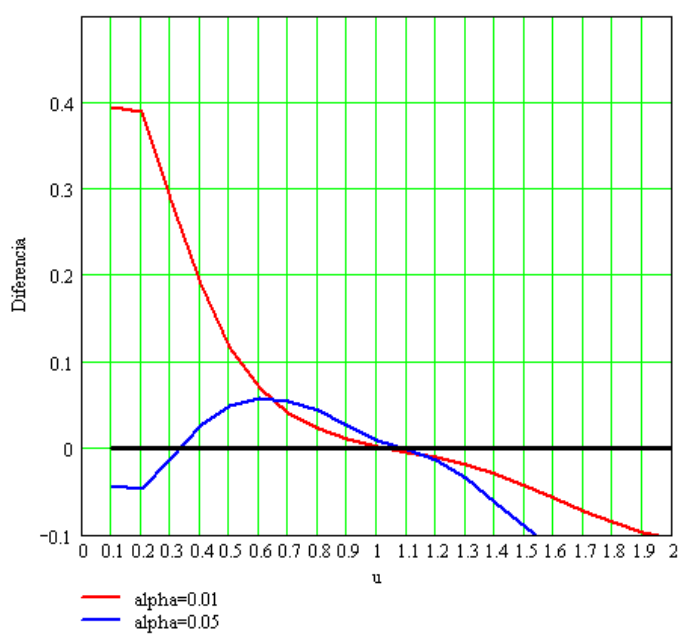

Fig. 7.1: $\operatorname{Pot}_{D S-U}-$ Pot $_{u}$ para $n=8$ y $u_{0}=1$

$\mathrm{Al}$ examinar las curvas de potencia, se observa que para un $\alpha$ pequeño $(\alpha=0.01)$, se aumenta más la potencia del gráfico $u$ en el tramo por debajo del valor de diseño $u_{0}=1$, al contrario de lo que sucede en el caso de $\alpha=0.05$. 


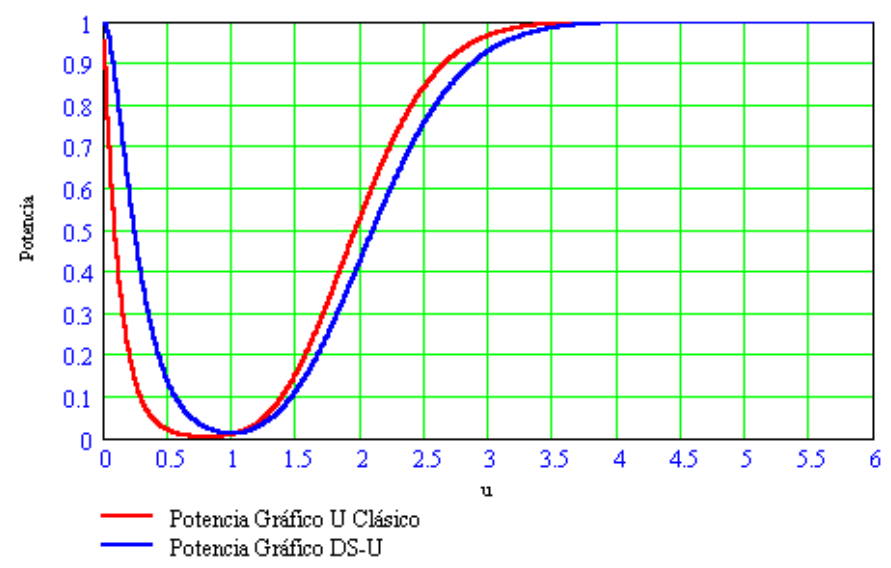

Fig. 7.2: Curva de potencia cuando $n=8, u_{0}=1$ y $\alpha=0.01$

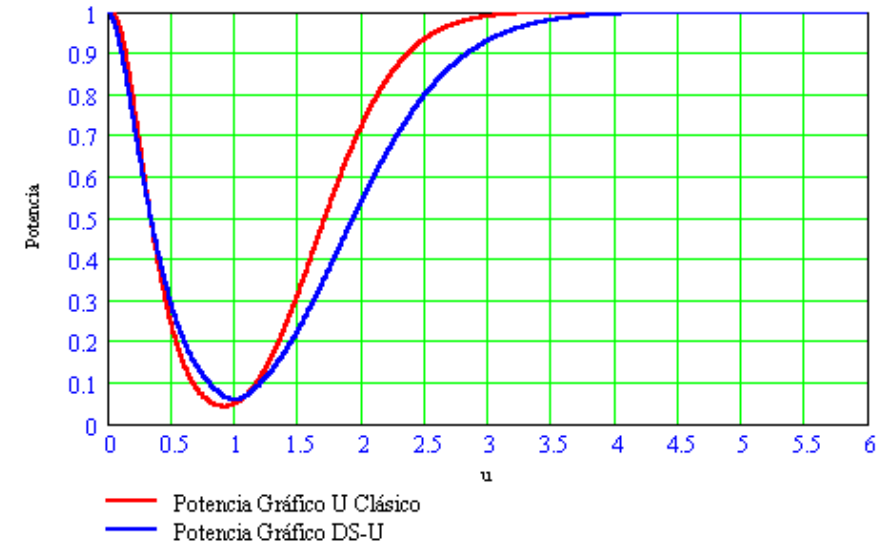

Fig. 7.3: Curva de potencia cuando $n=8, u_{0}=1$ y $\alpha=0.05$ 


\subsubsection{Comparación de la potencia de los gráficos de control u y $\boldsymbol{D S}-\boldsymbol{U}$ con $n=40$ y $u_{0}=1$}

Los casos experimentados en esta subsección son los que tienen como parámetros de entrada $n=40$ y $u_{0}=1$. Los resultados para distintos valores de $\alpha$ se han recogido en la tabla 7.3 .

\begin{tabular}{|c||c|c|c|c|c|c|c|c|}
\hline$\alpha_{\text {teórico }}$ & $L C I$ & $L A I$ & $L C I_{1}$ & $L A S$ & $L C S$ & $L C S_{1}$ & $n_{1}$ & $n_{2}$ \\
\hline \hline 0.01 & 24.4 & 24.8 & 51.9 & 53.9 & 62.6 & 80.1 & 39 & 76 \\
\hline \hline & $\alpha_{u}=9.131637 \cdot 10^{-3}$ & \multicolumn{2}{|c|}{$\alpha_{D S-U}=0.010958$} & $E[n]_{D S-U}=39.98$ \\
\hline \hline$\alpha_{\text {teórico }}$ & $L C I$ & $L A I$ & $L C I_{1}$ & $L A S$ & $L C S$ & $L C S_{1}$ & $n_{1}$ & $n_{2}$ \\
\hline \hline 0.05 & 10.4 & 27.5 & 81 & 51.3 & 53.3 & 69.9 & 39 & 9 \\
\hline \hline & \multicolumn{3}{|c|}{$\alpha_{u}=0.047396$} & \multicolumn{2}{c|}{$\alpha_{D S-U}=0.054277$} & $E[n]_{D S-U}=39.37$ \\
\hline \hline$\alpha_{\text {teórico }}$ & $L C I$ & $L A I$ & $L C I_{1}$ & $L A S$ & $L C S$ & $L C S_{1}$ & $n_{1}$ & $n_{2}$ \\
\hline \hline 0.0027 & 23.4 & 23.8 & 66.7 & 54.9 & 65.2 & 118.1 & 39 & 100 \\
\hline \hline & $\alpha_{u}=3.631613 \cdot 10^{-3}$ & \multicolumn{6}{|c|}{$\alpha_{D S-U}=4.357539 \cdot 10^{-3}$} & $E[n]_{D S-U}=39.89$ \\
\hline
\end{tabular}

Tabla 7.3: Solución obtenida para $n=40$ y $u_{0}=1$

La resolución del algoritmo genético no ha reducido sustancialmente el tamaño medio de muestra necesario para el gráfico $D S-U$, ya que en los tres experimentos es superior a $n=39$ unidades.

En cuanto al error de primera especie, también ha aumentado ligeramente, en torno a $10^{-3}$, por tanto queda determinar si la mejora de la curva de potencia es importante.

De la observación de la figura 7.4 se desprenden algunas conclusiones. En esta figura se representa la diferencia de potencias $\left(\left[\operatorname{Pot}_{D S-U}-P_{o t}\right]\right)$ en función de $u_{1}$. 
En primer lugar, las diferencias se dan de forma positiva en todo el rango de valores, a excepción del caso en que $\alpha=0.05$ y en $u_{1}=1.1$ del caso en que $\alpha=0.0027$.

Después se observa que las diferencias de potencia disminuyen a medida que aumenta el valor de $\alpha$, es decir, a menor $\alpha$, mayor es la potencia de detección del gráfico $D S-U$.

Además, a diferencia de los casos mostrados en el apartado anterior, el incremento de potencia respecto al gráfico $u$ llega a ser de un $20 \%$ en el caso de $\alpha=0.0027$, con lo que sí supone un incremento de la potencia importante.

Como conclusión se puede afirmar que cuando el tamaño de muestra $n$ es mayor y $\alpha$ disminuye, la potencia de detección del gráfico $D S-U$ propuesto aumenta notoriamente con respecto al gráfico u clásico para un valor de diseño $u_{0}=1$. En la representación de la curva de potencia de ambos gráficos que se observa en las figuras 7.5, 7.6 y 7.7 para distintos valores de $\alpha$ se puede concluir lo anteriormente mencionado. 
CAPÍTULO 7. COMPARACIÓN DE LA POTENCIA DEL GRÁFICO U FRENTE A LA POTENCIA DEL GRÁFICO DS - U EN $U_{1} \in\left[U_{I N F E R I O R}, U_{S U P E R I O R}\right]$

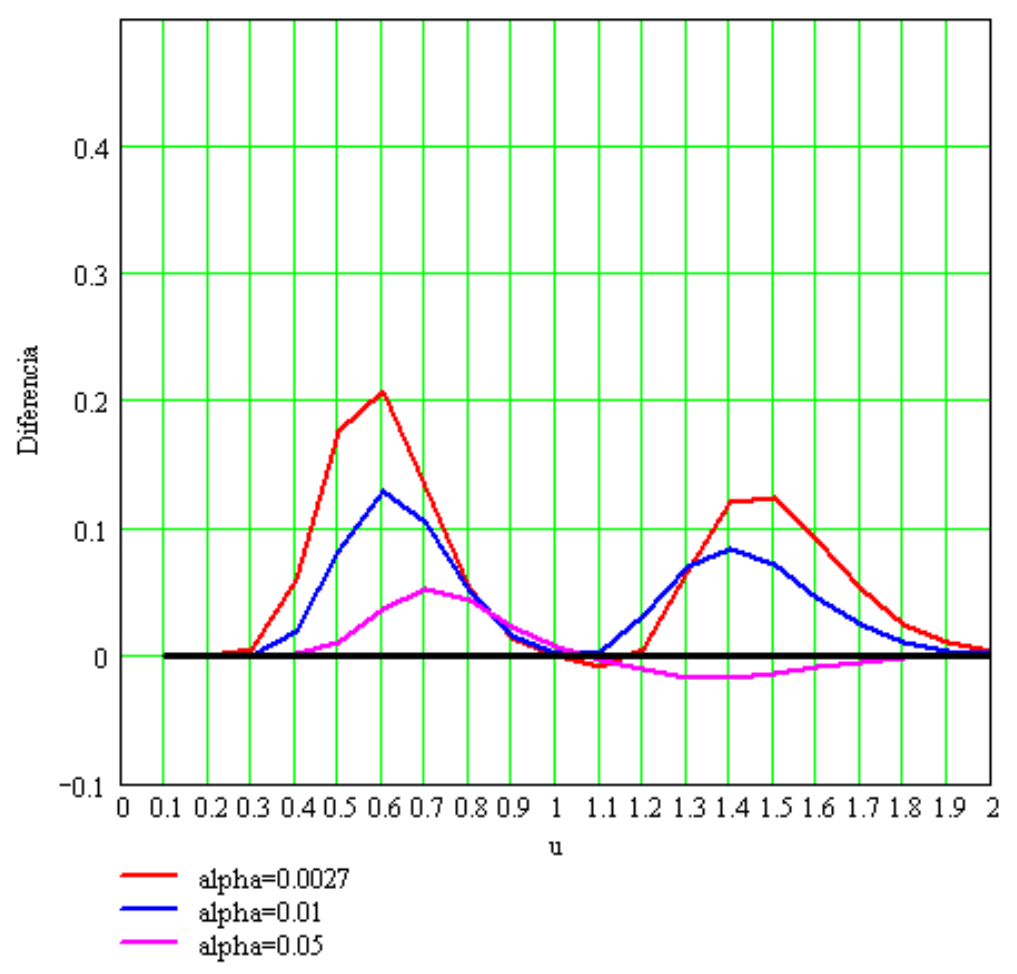

Fig. 7.4: Pot $_{D S-U}-$ Pot $_{u}$ para $n=40$ y $u_{0}=1$ 
CAPÍTULO 7. COMPARACIÓN DE LA POTENCIA DEL GRÁFICO U FRENTE A LA POTENCIA DEL GRÁFICO DS - U EN $U_{1} \in\left[U_{I N F E R I O R}, U_{S U P E R I O R}\right]$

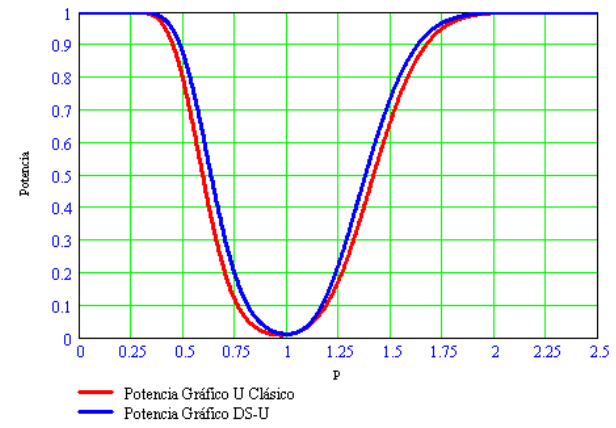

Fig. 7.5: $\alpha=0,01, n=40$ y $u_{0}=1$

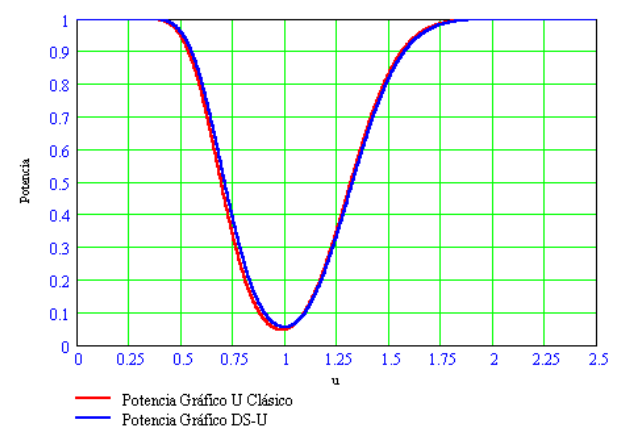

Fig. 7.6: $\alpha=0,05, n=40$ у $u_{0}=1$

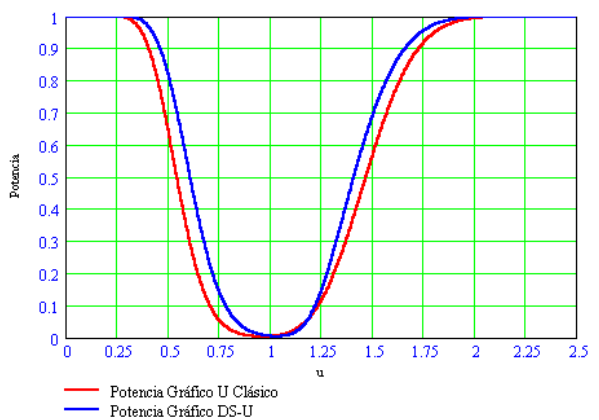

Fig. 7.7: $\alpha=0,0027, n=40$ y $u_{0}=1$ 


\subsubsection{Comparación de la potencia de los gráficos de control u y $D S-U$ con $n=8$ y $u_{0}=5$}

En este apartado se va a analizar la mejora aportada por el gráfico $D S-U$ para tres casos, en los que los valores de diseño del gráfico $u$ son $n=8 \mathrm{y}$ $u_{0}=5$. Se comparará el efecto que provoca la disminución de $\alpha$ en la mejora de la potencia.

Estos últimos casos de estudio del capítulo son para tres gráficos con los siguientes parámetros: $n=8$ y $u_{0}=5$, así como comparando 3 valores de $\alpha$ distintos. Los resultados obtenidos para los parámetros del nuevo gráfico $D S-U$ se reflejan en la tabla 7.4 .

\begin{tabular}{|c|c|c|c|c|c|c|c|c|}
\hline$\alpha_{\text {teórico }}$ & $L C I$ & $L A I$ & $L C I_{1}$ & $L A S$ & $L C S$ & $L C S_{1}$ & $n_{1}$ & $n_{2}$ \\
\hline 0.01 & 21.1 & 21.9 & 99.4 & 46.5 & 57.8 & 160 & 7 & 29 \\
\hline & \multicolumn{3}{|c|}{$\alpha_{u}=9.131637 \cdot 10^{-3}$} & \multicolumn{3}{|c|}{$\alpha_{D S-U}=0.010834$} & \multicolumn{2}{|c|}{$E[n]_{D S-U}=7.87$} \\
\hline$\alpha_{\text {teórico }}$ & $L C I$ & $L A I$ & $L C I_{1}$ & $L A S$ & $L C S$ & $L C S_{1}$ & $n_{1}$ & $n_{2}$ \\
\hline \multirow[t]{2}{*}{0.05} & 25.5 & 25.6 & 92.3 & 45.8 & 54.5 & 130.2 & 7 & 24 \\
\hline & \multicolumn{3}{|c|}{$\alpha_{u}=0.047396$} & \multicolumn{3}{|c|}{$\alpha_{D S-U}=0.056858$} & \multicolumn{2}{|c|}{$E[n]_{D S-U}=7.99$} \\
\hline$\alpha_{\text {teórico }}$ & $L C I$ & $L A I$ & $L C I_{1}$ & $L A S$ & $L C S$ & $L C S_{1}$ & $n_{1}$ & $n_{2}$ \\
\hline \multirow[t]{2}{*}{0.0027} & 16.2 & 16.9 & 72.2 & 37.5 & 52 & 138.2 & 6 & 22 \\
\hline & \multicolumn{3}{|c|}{ 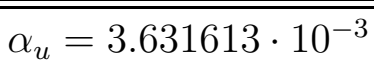 } & \multicolumn{3}{|c|}{$\overline{\alpha_{D S-U}}=4.355987 \cdot 10^{-3}$} & \multicolumn{2}{|c|}{ 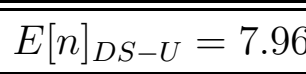 } \\
\hline
\end{tabular}

Tabla 7.4: Solución obtenida para $n=8$ y $u_{0}=5$

El tamaño medio de muestra ha quedado fijado en valores próximos al valor de diseño del gráfico $u$, y con el valor de $\alpha$ sucede lo mismo que en los dos apartados anteriores, con un pequeño aumento de $\alpha_{D S-U}$ con respecto a $\alpha_{u}$. 
Para determinar si el incremento de la potencia del gráfico propuesto supone un avance en comparación con el gráfico $u$, hay que examinar la figura 7.8. Se concluye lo siguiente:

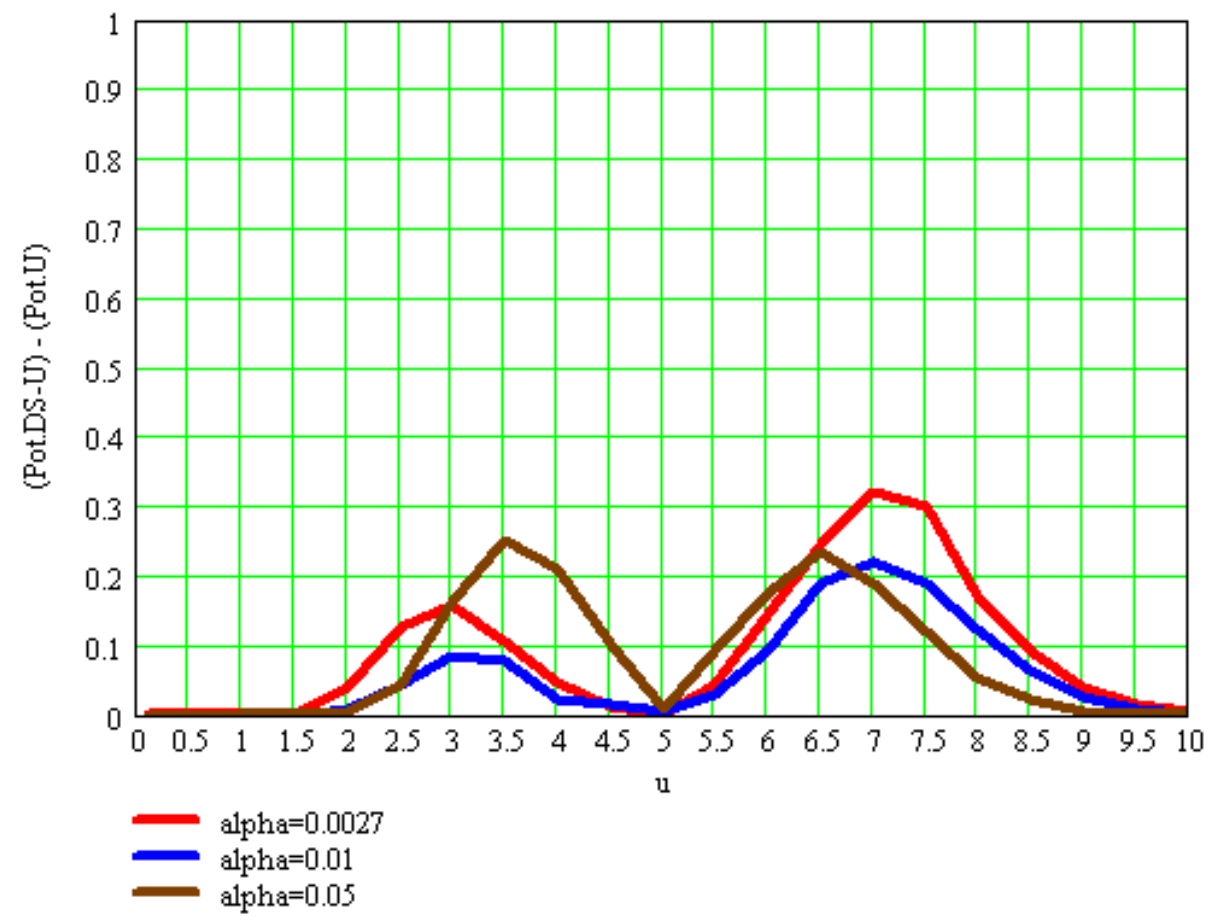

Fig. 7.8: Pot $_{D S-U}-$ Pot $_{u}$ para $n=8$ y $u_{0}=5$

- El patrón de mejora de la potencia en el tramo de valores superior al valor de diseño $u_{0}$ queda claramente diferenciado del patrón de dicha mejora en el tramo inferior de $u_{0}$.

- Para un mismo $n$ y $u_{0}$ tenemos:

1. En el tramo de valores inferior al valor de diseño: cuanto mayor es $\alpha$, mejor es la potencia obtenida para el gráfico $D S=U$ frente al gráfico $u$. 
2. En el tramo de valores superior al valor de diseño

a) Cuanto menor es $\alpha$, mayor es la mejora de la potencia obtenida.

b) Los valores de las tres curvas de diferencia de potencia son muy distintos, teniendo distancia pico entre ellas de 0.1 .

- Comparando con la figura 7.1, el incremento del valor de diseño a $u_{0}=5$ ha supuesto una mejora sustancial en el comportamiento de la curva de potencia. 


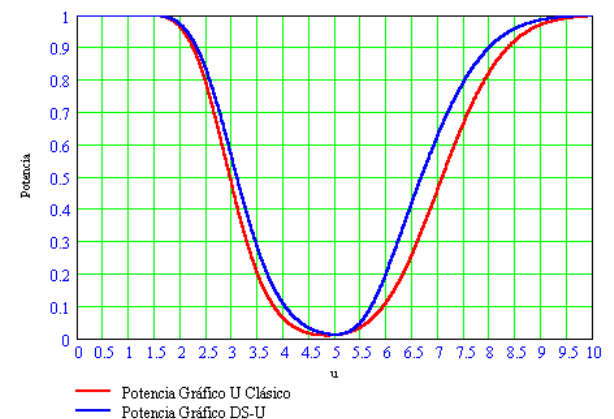

Fig. 7.9: Curva de potencia para $\alpha=0,01, n=8$ y $u_{0}=5$

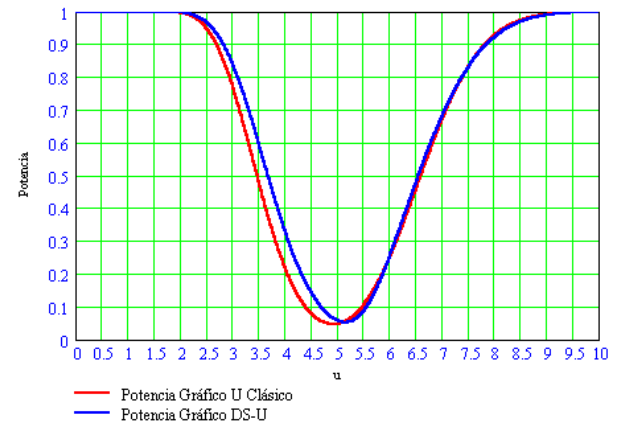

Fig. 7.10: Curva de potencia para $\alpha=0,05, n=8$ y $u_{0}=5$

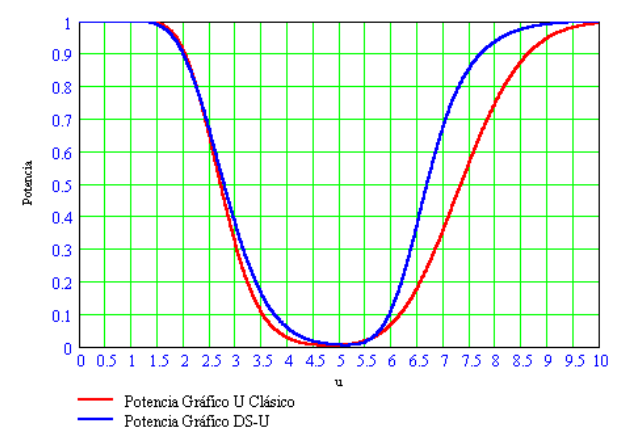

Fig. 7.11: Curva de potencia para $\alpha=0,0027, n=8$ y $u_{0}=5$ 


\subsection{Conclusiones}

- Parámetros: $n=8$ y $u_{0}=1$

1. En ninguno de los casos estudiados se ha conseguido optimizar en todos y cada uno de los puntos del intervalo, aunque en el caso de que $\alpha$ sea menor, la mejora es mayor, y además se consigue optimizar en todo el tramo inferior al valor de diseño $u_{0}=1$. En las curvas de potencia se detecta que para un $\alpha$ pequeño $(\alpha=0.01)$, se aumenta más la potencia del gráfico $u$ en el tramo por debajo del valor de diseño $u_{0}=1$, al contrario de lo que sucede en el caso de $\alpha=0.05$.

2. El tamaño medio de muestra es superior a $n=7$ en ambos casos, con lo que la reducción del tamaño de muestra no es considerable con respecto al gráfico clásico $u$.

- Parámetros : $n=40$ y $u_{0}=1$

1. La resolución del algoritmo genético no ha reducido sustancialmente el tamaño medio de muestra necesario para el gráfico $D S-U$, ya que en los tres experimentos es superior a $n=39$ unidades.

2. En cuanto al error de primera especie, también ha aumentado ligeramente, en torno a $10^{-3}$, por tanto queda determinar si la mejora de la curva de potencia es importante.

3. De forma general, las diferencias de potencia entre el gráfico $D S-U$ y $u$ son positivas en todo el rango de valores.

4. Se observa que las diferencias de potencia disminuyen a medida que aumenta el valor de $\alpha$, es decir, a menor $\alpha$, mayor es la potencia de detección del gráfico $D S$ - $U$. 
5. Además, a diferencia de los casos mostrados en el apartado anterior, el incremento de potencia respecto al gráfico $u$ llega a ser de un $20 \%$ en el caso de $\alpha=0.0027$, con lo que sí supone un incremento de la potencia importante.

6. Se puede afirmar que cuando el tamaño de muestra $n$ es mayor y $\alpha$ disminuye, la potencia de detección del gráfico $D S-U$ propuesto aumenta notoriamente con respecto al gráfico $u$ clásico para un valor de diseño $u_{0}=1$.

- Parámetros: $n=8$ y $u_{0}=5$

1. El tamaño medio de muestra ha quedado fijado en valores próximos al valor de diseño del gráfico $u$, y con el valor de $\alpha$ sucede lo mismo que en los dos apartados anteriores, con un pequeño aumento de $\alpha_{D S-U}$ con respecto a $\alpha_{u}$.

2. Ahora se ve que:

- El patrón de mejora de la potencia en el tramo de valores superior al valor de diseño $u_{0}$ queda claramente diferenciado del patrón de dicha mejora en el tramo inferior de $u_{0}$.

a) En el tramo de valores inferior al valor de diseño: cuanto mayor es $\alpha$, mejor es la potencia obtenida para el gráfico $D S-U$ frente al gráfico $u$.

b) En el tramo de valores superior al valor de diseño

1) Cuanto menor es el valor de $\alpha$, mayor es la mejora de la potencia obtenida.

2) Los valores de las tres curvas de diferencia de potencia son distintos, teniendo distancia pico entre ellas de 0.1 .

- Comparando con el primer caso estudiado en que $n=8$ y $u_{0}=1$, el incremento del valor de diseño a $u_{0}=5$ ha supuesto 
una mejora importante en el comportamiento de la curva de potencia.

\subsection{Ejemplo}

A continuación se va a mostrar un ejemplo de aplicación a un proceso concreto, que ya ha sido analizado en el ejemplo del capítulo 6 para un valor de $u_{1}$.

En la mencionada fábrica dedicada a la inyección de plásticos se tiene un proceso de fabricación en una inyectora en el que se considera un número de defectos por unidad de muestreo de $u_{0}=1.3 \mathrm{y}$ se busca una capacidad de detección lo más alta posible para el intervalo de valores: $u_{1} \in[0.8,1.8]$. En la imagen de la figura 7.12 se puede observar una vista de dicha máquina.

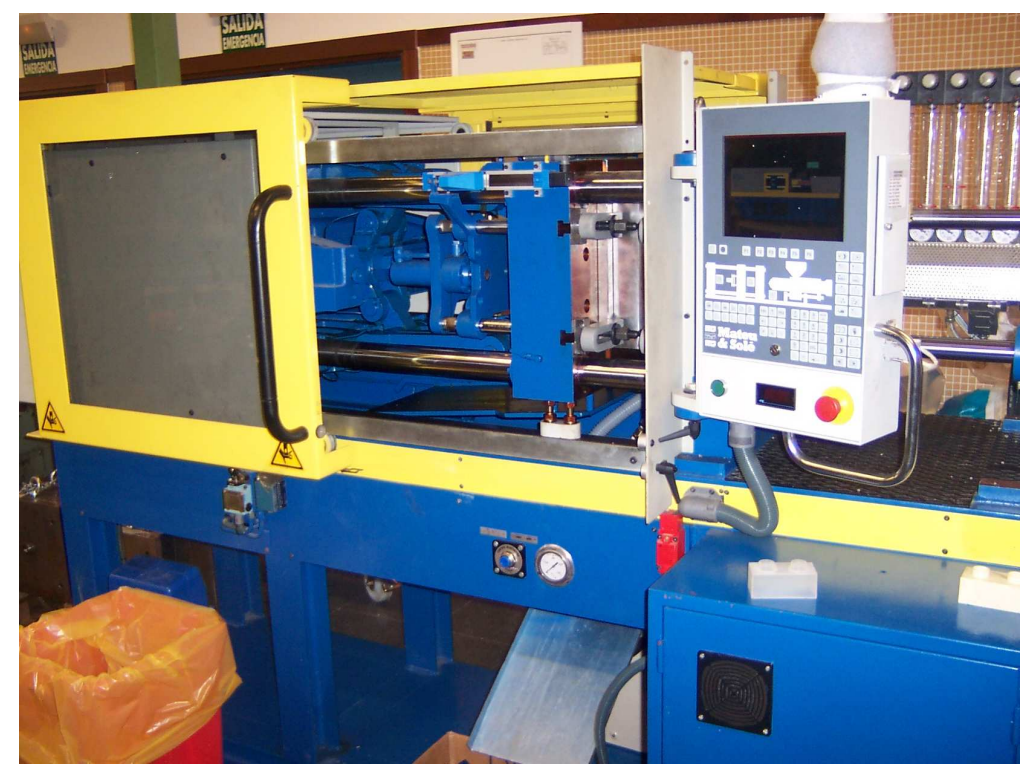

Fig. 7.12: Máquina Inyectora 
El control se está llevando con un gráfico u clásico cuyas características son:

- $\mathrm{n}=10$

- $\alpha_{t}=0.01$

- $L C S=2.2284$

- $L C I=0.3715$

En esta ocasión se introducen los parámetros en el software programado para esta Tesis Doctoral para intentar mejorar la potencia de detección en el intervalo $u_{1} \in[0.8,1.8]$.

Los límites calculados para el nuevo gráfico $D S-U$ son los representados en la figura 7.13.

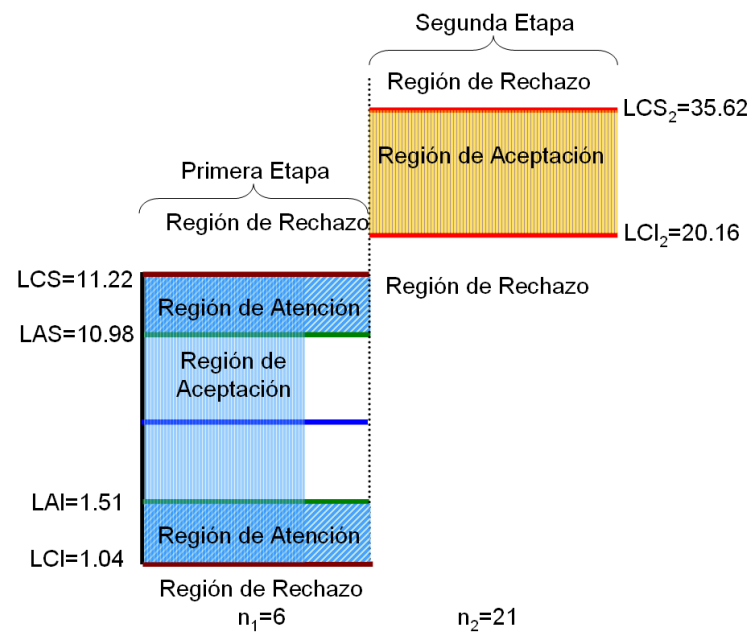

Fig. 7.13: Gráfico de Control DS-U 
La curva de la diferencia de potencias entre el gráfico $D S-U$ y el gráfico $u$ : $\operatorname{Pot}_{D S-U}-$ Pot $_{u}$ se ha representado en la gráfica 7.14.

Se puede destacar de la observación de la gráfica 7.14 que se obtiene diferencia de potencias positiva en todo el tramo especificado, $[0.8,1.8]$, es decir, con el gráfico $D S-U$ se obtiene mejor potencia que con el gráfico u en todos los valores de $u_{1}$ incluidos en dicho intervalo. El gráfico diseñado para este ejemplo, mejora tanto en el Tramo Superior como en el Tramo Inferior.

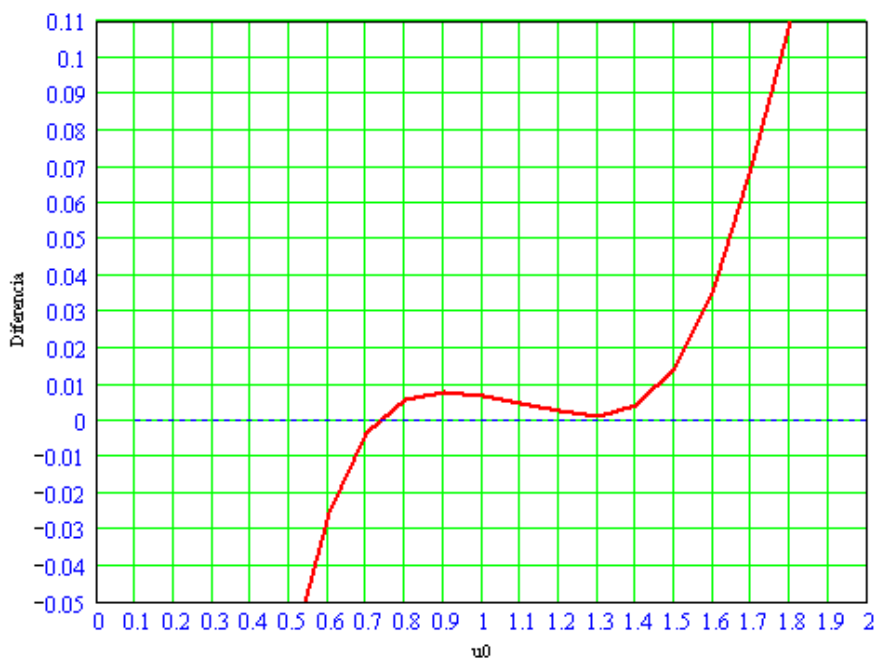

Fig. 7.14: $\operatorname{Pot}_{D S-U}-\operatorname{Pot}_{u}$ para $n=10, u_{0}=1.3$ y $\alpha_{t}=0.01$

En esta gráfica (7.14) se puede observar que la curva de potencia del gráfico $u$ es en general mucho mejor que la del gráfico $D S-U$ en valores de $u_{1}>0.8$, que el límite inferior del intervalo que se ha propuesto mejorar; y además, esta mejora se dispara a partir de $u_{1}=1.4$. Por tanto, se consigue el objetivo con una propuesta de gráfico $D S-U$ que es superior en prestaciones al gráfico $u$, no sólo en el intervalo indicado, sino a partir del límite inferior de dicho intervalo, $u_{1}=8$, extendiendo la mejora obtenida a valores superiores al límite superior del intervalo, $u_{1}=1.8$. 
En las figuras 7.15 y 7.16 se muestran las dos curvas de potencia, en rojo la curva de potencia del gráfico $u$ y de color azul la curva de la potencia del gráfico $D S$ - $U$. Se puede observar claramente, la mejora conseguida con el nuevo gráfico $D S-U$ propuesto.

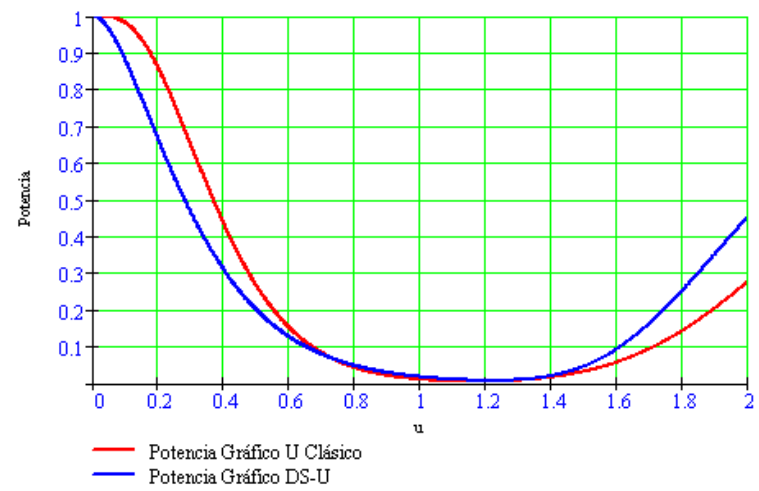

Fig. 7.15: Curvas de potencia para $u_{1} \in[0.8,1.8]$

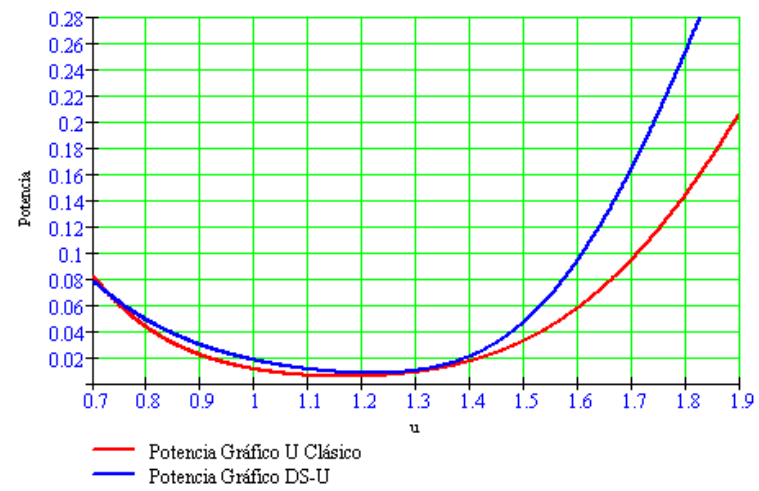

Fig. 7.16: Detalle ampliado de la gráfica de las curvas de potencia para $u_{1} \in[0.8,1.8]$ 


\section{Capítulo 8}

\section{Conclusiones y Resultados}

En este capítulo se resumen las conclusiones y los resultados que se han obtenido a lo largo de esta Tesis Doctoral, que se enumeran a continuación, clasificados por apartados:

\section{Recomendaciones generales.}

1. Se ha demostrado que el nuevo gráfico $D S-U$ mejora la potencia del gráfico $u$.

2. Dicha mejora en la potencia no es para cualquier caso, sólo para determinados tamaños de muestra $n$, valores de $\alpha$ y $u_{0}$.

3. Se aporta a los responsables de fabricación una herramienta que les permite el diseño de un gráfico de control más potente para controlar desviaciones a un determinado valor. 


\section{Conclusiones sobre los gráficos DS-U}

Se dividen las conclusiones en función de qué se ha intentado mejorar:

- Mejora en un valor $u_{1}$

- En general se consigue mejorar en todos los valores de $u_{1}$, a excepción de cuando $u_{0}$ y $n$ son valores altos (en los ejemplos analizados: $n=40$ y $u_{0}=5$ ), en cuyo caso sólo se mejora si $u_{1}>u_{0}$, denominado Tramo Superior. Este Tramo Superior consiste en que el valor en el que se intenta obtener una mejor potencia de detección es superior al número de defectos por unidad $u_{0}$, y es en estos valores en los que sí se consigue incrementar la potencia del gráfico $u$ para dicha excepción.

- El gráfico $D S-U$ ofrece interesantes ventajas para tamaños de muestra pequeños, y también para valores de $u_{0}$ pequeños, en los que el porcentaje de mejora del gráfico $D S$ - $U$ frente al gráfico $u$ son muy elevados en algunos casos.

- El valor de $\alpha$ influye en cuánto mejora el gráfico. El patrón de comportamiento no es igual en todos los casos, y está ampliamente explicado en el capítulo 6.

- Mejora en un intervalo $\left[u_{\text {inferior }}, u_{\text {superior }}\right]$

- En general, el tamaño medio de muestra, $E[n]$, se reduce como máximo en 1 unidad en todos los ejemplos estudiados. El gráfico $D S-U$ reduce ligeramente el tamaño medio de muestra con respecto al gráfico $u$, pero hay que tener en cuenta que los valores de $\alpha$ no son idénticos en los gráficos $u$ y $D S-U$.

- No se consigue optimizar en todos y cada uno de los valores de $u_{1}$ cuando se trata de valores pequeños de $n$ y $u_{0}$, caso en el que sólo 
se mejora en el tramo inferior $\left(u_{1}<u_{0}\right)$, es decir, para los valores del intervalo $\left[u_{\text {inferior }}, u_{\text {superior }}\right]$ que son menores que el valor de diseño $u_{0}$.

- A mayor valor de $n$, y a su vez, a mayor valor de $u_{0}$, mejor es el incremento de la potencia ofrecida por el gráfico $D S-U$.

- Para valores de $u_{0}$ pequeños se tiene que a menor $\alpha$, la potencia de detección del gráfico $D S-U$ propuesto aumenta notoriamente con respecto al gráfico $u$ clásico. Para valores de $u_{0}$ mayores, el incremento de la potencia de detección del gráfico $D S-U$ es mayor que cuando $u_{0}$ pequeño.

Por otra parte, se proponen las siguientes líneas de investigación futura como consecuencia de la realización de esta Tesis Doctoral:

1. Comparación de la metodología de Doble Muestreo frente a otras propuestas de mejora por parte de otros autores como (Chan, Xie, and Goh 2000).

2. Estudiar el comportamiento de esta metodología aplicada al gráfico $p$.

3. De cara a una posible aplicación práctica, sería interesante la realización de una aplicación informática que permita la adquisición de datos directamente de una determinada máquina de producción, y que elabore a su vez el gráfico de control, facilitando así las tareas del responsable de calidad. 



\section{Bibliografía}

Aguirre-Torres, Victor, and Daniel Reyes-López. 1999. "Run Sum Charts for both $\bar{X}$ and R." Quality Engineering 12 (1): 7-12.

Bäck, Thomas. 1996. Evolutionary algorithms in theory and practice. Evolution strategies, evolutionary programming, genetic algorithms. Oxford University Press.

- 1998. Optimization by Means of Genetic Algorithms. Oxford University Press.

Bäck, Thomas, and Hans-Paul Schwefel. 1993. "An Overview of Evolutionary Algorithms for Parameter Optimization." Evolutionary Computation 1 (1): 1-23.

Bryce, G.Rex, Marie A. Gaudard, and Brian L. Joiner. 1997. "Estimating the standard deviation for individuals control charts." Quality Engineering 10 (2): 331-341.

Carot, V. 1998. Control Estadístico de la Calidad. Valencia: Servicio de Publicaciones de la U.P.V. - 98.4007.

Carot, V., J.M. Jabaloyes, and T. Carot. 2002. "Combined double sampling and variable sampling interval $\bar{X}$ chart." International Journal of Production Research 40 (9): 2175-2186.

Chakraborti, S., and S.W. Human. 2006. "Parameter Estimation and Per- 
Bibliografía

formance of the $p$-Chart for Attributes Data." IEEE Transactions on Reliability, no. 55:559-566.

Champ, Charles W., and Allison Jones. 2004. "Designing Phase I $\bar{X}$ Charts with Small Sample Sizes." Quality Reliability Engineering International, no. 20:497-510.

Champ, Charles W., and W.H. Woodall. 1987. "Exact Results for Shewhart Control Charts with Supplementary Runs Rules." Technometrics, no. 29:393-399.

Chan, L.Y., C.D. Lai, M. Xie, and T.N. Goh. 2003. "A two-stage decision procedure for monitoring processes with low fraction nonconforming." European Journal of Operational Research, no. 150:420-436.

Chan, L.Y., M. Xie, and T.N. Goh. 2000. "Cumulative quantity control charts for monitoring production processes." International Journal of Production Research 38 (2): 397-408.

Che, Yi-Hau, and Hung Chen. 2000. "A unified approach to regression analysis under double-sampling designs." Journal R.Statisc. Society 62 (3): 449-460.

Chen, Gemai, and Smiley W. Cheng. 1998. "The exact u chart can be obtained using simple adjustments." Statistic and Probability Letters 37 (4): 357-365.

Costa, A.F.B. 1994. "X $\bar{X}$ Charts with Variable Sampling Size." Journal of Quality Technology 26 (3): 155-163.

—. 1997. " $\bar{X}$ Chart with Variable Sampling Size and Sampling Intervals." Journal of Quality Technology 29 (2): 197-204.

—. 1999. "X X Charts with Variable Parameters." Journal of Quality Technology 31 (4): 408-416.

—. 2001. "Graficos $\bar{X}$ e $\mathrm{R}$ com amostragens em dois estágios." XXXIII SBPO. 
Bibliografía

Costa, A.F.B., and M.S. De Magalhâes. 2005. "Economic design of twostage $\bar{X}$ Charts: the Markov chain approach." International Journal of Production Economics 95:9-20.

Costa, A.F.B., M.S. De Magalhâes, and Eugenio K. Epprecht. 2005. "The Non-Central Chi-Square Chart with Double Sampling." Brazilian Journal of Operations and Production Management 2 (3): 21-37.

Costa, A.F.B., and Marcela A.G. Machado. 2007. "Synthetic control charts with two-stage sampling for monitoring bivariate processes." Pesquisa Operacional 27:117-130.

Costa, A.F.B., and Abdur Rahim. 2004. "Joint $\bar{X}$ Charts and R charts with two stage samplings." Quality and Reliability Engineering International 20 (7): 699-708.

Costa, A.F.B., and M.A. Rahim. 2006. "The non-central chi-square chart with two stage samplings." European Journal of Operational Research 171:64-73.

Croasdale, P. 1974. "Control Charts for a Double-Sampling Scheme Based on Average Production Run Lengths." International Journal of Production Research 12:585-592.

Crowder, S.V. 1987. "A Simple Method for Studying Run-Length Distributions of Exponentially Weighted Moving Average Charts." Technometrics 29:401-407.

Daudin, J.J. 1992. "Double Sampling X Charts." Journal of Quality Technology 24 (2): 78-87.

Davis, Robert B., and William H. Woodall. 2002. "Evaluating and Improving the Synthetic Control Chart." Journal of Quality Technology 34, no. 2 (April).

Elam, Matthew E., and Kenneth E. Case. 2001. "A Computer Program 
Bibliografía

to Calculate Two-Stage Short-Run Control Chart Factors for (X-bar,R) Charts." Quality Engineering 14 (1): 77-102.

Epprecht, E.K., and A.F.B. Costa. 2001. "Adaptive sample size control charts for attributes." Quality Engineering 13 (3): 465-473.

Falkenauer, E. 1998. Genetic Algorithms and Grouping problems. John Wiley and sons.

Fang, Yue. 2003. "c-charts, X-charts, and the Katz Family of Distributions." Journal of Quality Technology 35 (1): 104-114.

Goldberg, D. 1989. Genetics Algorithms in Search, Optimization and Machine Learning. Addison Wesley.

Goldfarb, Heidi B., Connie Borror, Douglas C. Motgomery, and Christine M. Anderson-Cook. 2005. "Using Genetic Algorithms to Generate MixtureProcess Experimental Designs Involving Control and Noise Variables." Journal of Quality Technology 37 (1): 60-74.

Hamada, Michael. 2002. "Bayesian tolerance interval control limits for attributes." Quality and Reliability Engineering International 18:45-52.

He, D., A. Grigoryan, and M. Sigh. 2002. "Design of double- and triplesampling $\bar{X}$ control charts using genetic algorithms." International Journal of Production Research 40 (6): 1387-1404.

He, David, and Arsen Grigoryan. 2002. "Construction of Double Sampling s-Control Charts for agile manufacturing." Quality and Reliability Engineering International 18:343-355.

- 2003. "An improved double sampling s chart." International Journal of Production Research 41 (12): 2663-2679.

Heredia-Langner, Alejandro, W.M. Carlyle, D.C. Montgomery, C.M. Borror, and G.C. Runger. 2003. "Genetic Algorithms for the Construction of D-Optimal Designs." Journal of Quality Technology 35 (1): 28-46. 
Bibliografía

Jolayemi, Joel K. 2002. "Statistical design of np-charts with multiple control regions." International Journal of Production Research 40 (11): 25912608.

Kaya, Ishan, and Orhan Engin. 2007. "A new approach to define sample size at attributes control chart in multistage processes: An application in engine piston manufacturing process." Materials Processing Technology 183:38-48.

Khoo, Michael B.C. 2004. "Poisson Moving Average Versus c Chart for Nonconformities." Quality Engineering 16 (4): 525-534.

Klein, Morton. 2000. "Two Alternatives to the Shewhart X-bar Control Chart." Journal of Quality Technology 32 (4): 427-431.

Koza, J.R. 1999. AG. Morgan Kauffman Publishers.

Lin, Yu-Chang, and Chao-Yu Chou. 2005. "Adaptive $\bar{X}$ Control Charts with Sampling at Fixed Times." Quality and Reliability Engineering International 21:163-175.

Lucas, James M., Darwin J. Davis, and Erwin M. Saniga. 2006. "Detecting improvement using Shewhart attribute control charts when the lower control limit is zero." IIE Transactions 38:699-709.

Lucas, J.M., and M.S. Saccucci. 1990. "Exponentially Weighted Moving Average Control Schemes: Properties and Enhancements." Technometrics $32: 1-12$.

Luo, Hua, and Zhang Wu. 2002. "Optimal np Control Charts with Variable Sample Sizes or Variable Sampling Intervals." Economic Quality Control 17 (1): 39-61.

Magalhâes, Maysa S., Antonio F.B. Costa, and K. Epprecht. 2002. "Constraines optimization model for the design of an adaptive $\bar{X}$ chart." Int. Journal Prod.Res 40 (13): 3199-3218. 
Bibliografía

Martorell, S., S. Carlos, A. Sánchez, and V. Serradell. 2000. "Constrained optimization of test intervals using a steady-state genetic algorithm." Reliability Engineering and System Safety 67:215-232.

Montgomery, Douglas C. 1991. Control Estadístico de la Calidad. Grupo Editorial Iberoamérica.

Page, E.S. 1954. "Continuous inspection schemes." Biometrika 41:100-115.

Prahbu, S.S., G.C. Runger, and J.B. Keats. 1993. "An Adaptive Sample Size $\bar{X}$ Chart." International Journal of Production Research 31:2895-2909.

Quesenberry, Charles P. 1993. "The Effect of Sample Size on Estimated Limits for X-bar and X Control Charts." Journal of Quality Technology 25 (4): $237-247$.

—. 1995. "Geometric $Q$ Charts for High Quality Processes." Journal of Quality Technology 27 (4): 304-315.

—. 2001. "On the Two-Stage p-Chart." Quality Engineering 14 (2): $253-256$.

Rahim, M.A., and Antonio F.B. Costa. 2000. "Joint economic design of $\mathrm{x}$-bar and $\mathrm{R}$ charts under Weibull shock models." International Journal of Production Research 38 (13): 2871-2889.

Reynolds Jr, Marion R., Raid W. Amin, Jesse C. Arnold, and Joel A. Nachlas. 1988. " $\bar{X}$ Charts with Variable Sampling Intervals." Technometrics 30 (2): 181-192.

Roberts, S.W. 1959. "Control-Charts-Tests based on geometric moving averages." Technometrics 1:239-250.

Rudisill, Frank, Lewis A. Litteral, and Don Walter. 2004. "Modified u Charts for Monitoring and Controlling Poisson Attribute Processes." Quality Engineering 16 (4): 637-642.

Runger, George C., and Pignatiello Jr. Joseph J. 1991. "Adaptive Sampling for Process Control." Journal of Quality Technology 23 (2): 135-155. 
Bibliografía

Ryan, Tomas P., and Neil C. Schwertman. 1997. "Optimal Limits for Attributes Control Charts." Journal of Quality Technology 29 (1): 86-98.

Samuel, Thomas R., Pignatiello Jr. Joseph J., and James A. Calvin. 1998. "Identifying the time of a step change with X-bar Control Charts." Quality Engineering 10 (3): 521-527.

Schwertman, Neil C., and Thomas P. Ryan. 1999. "Using Dual np-charts to detect changes." Quality and Reliability Engineering International $15: 317-320$.

Schwertman, Neil C., and Tomas P. Ryan. 1997. "Implementing Optimal Attributes Control Charts." Journal of Quality Technology 29 (1): 99104.

Shewhart, W.A. 1931. Economic Control of Quality of Manufactured Product. D. Van Nostrand Company, Inc., Toronto, Canada.

Shore, Haim. 2000. "General control charts for variables." International Journal of Production Research 38 (8): 1875-1897.

Steiner, S.H. 1999. "Confirmation sample control charts." International Journal of Production Research 37 (4): 737-748.

Tagaras, George. 1998. "A Survey of Recent Developments in the Design of Adaptive Control Charts." Journal of Quality Technology 30 (3): $212-231$.

Wang, Zhigang, and Wen-xiu Ma. 2003. "Design of an optimum adaptive control chart for attributes." Proceedings of the 2003 Systems and Information Engineering Design Symposium. Matthew H.Jones, Barbara E. Tawney, and K.Preston White, Jr., eds., 213-219.

Wu, Zhang, and Hua Luo. 2003. "Three-triplet np control charts." European Journal of Operational Research, no. 149:614-624.

—. 2004. "Optimal Design of the Adaptive Sample Size and Sam- 
pling Interval np Control Chart." Quality and Reliability Engineering International 20:553-570.

Wu, Zhang, Hua Luo, and Xiaolan Zhang. 2006. "Optimal np control chart with curtailment." European Journal of Operational Research, no. 174:1723-1741.

Xie, M., T.N. Goh, and X.S. Lu. 1998. "A comparative study of CCC and CUSUM charts." Quality and Reliability Engineering International 14:339-345.

Yang, Zhenlin, Min Xie, Vellaisamy Kuralmani, and Kwok-Leung Tsui. 2002. "On the Performance of Geometric Charts with Estimated Control Limits." Journal of Quality Technology 34, no. 4.

Zimmer, Lora S., Douglas C. Montgomery, and George C. Runger. 1998. "Evaluation of a three-state adaptive sample size $\bar{X}$ control chart." International Journal of Production Research 36 (3): 733-743.

— 2000. "Guidelines for the application of adaptive control charting schemes." International Journal of Production Research 38 (9): 19771992. 
Apéndice A

Comparación de la potencia del gráfico $u$ frente a la potencia del gráfico $D S-U$ en un valor $u_{1}$

A.1. Comparación de los gráficos de control $u$ y $\boldsymbol{D} \boldsymbol{S}-\boldsymbol{U} \operatorname{con} n=8, u_{0}=1$ y $\alpha_{t}=0.01$ 
APÉNDICE A. COMPARACIÓN DE LA POTENCIA DEL GRÁFICO U FRENTE A LA POTENCIA DEL GRÁFICO DS-U EN UN VALOR U

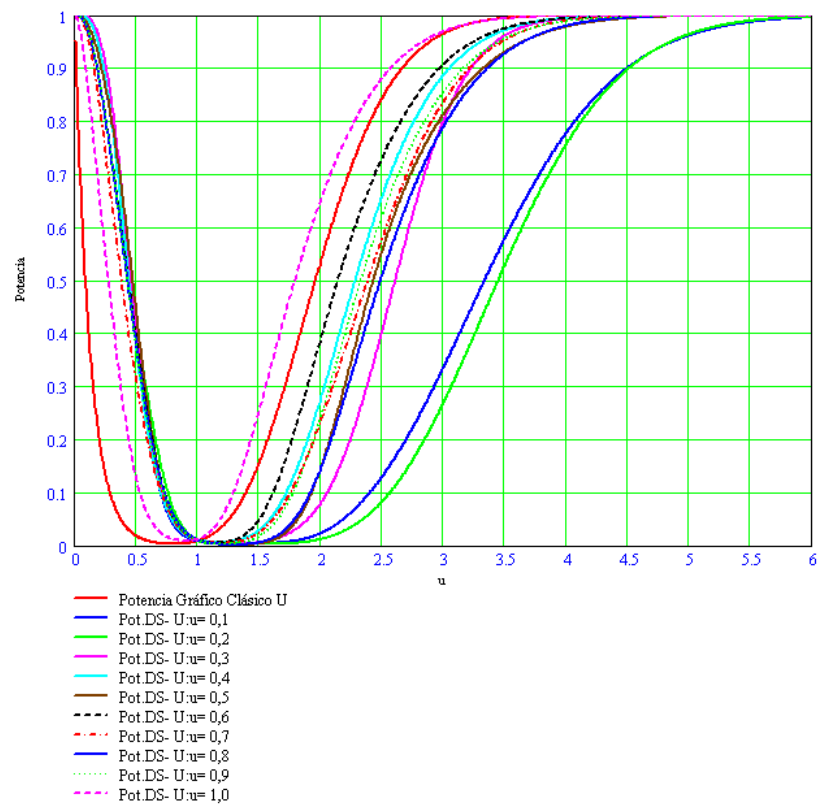

(a) $u_{1} \in[0.1,1.0]$
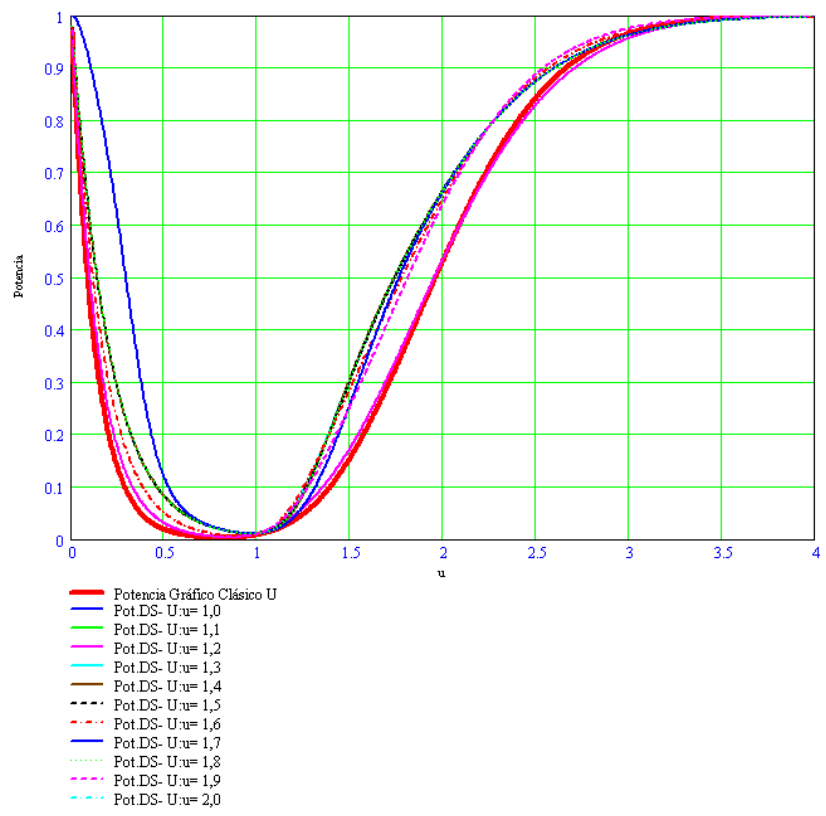

(b) $u_{1} \in[1.1,2.0]$

Fig. A.1: Curvas de potencia en distintos valores $u_{1}$ 
APÉNDICE A. COMPARACIÓN DE LA POTENCIA DEL GRÁFICO U FRENTE A LA POTENCIA DEL GRÁFICO DS-U EN UN VALOR U

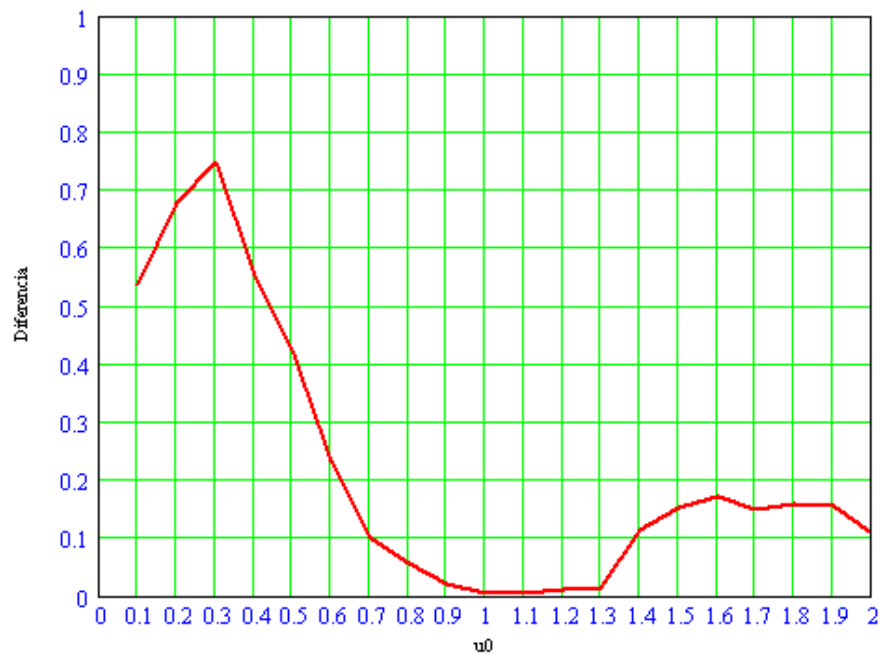

Fig. A.2: $\operatorname{Pot}_{D S-U}-\operatorname{Pot}_{u}$ para $n=8, u_{0}=1$ y $\alpha_{t}=0.01$

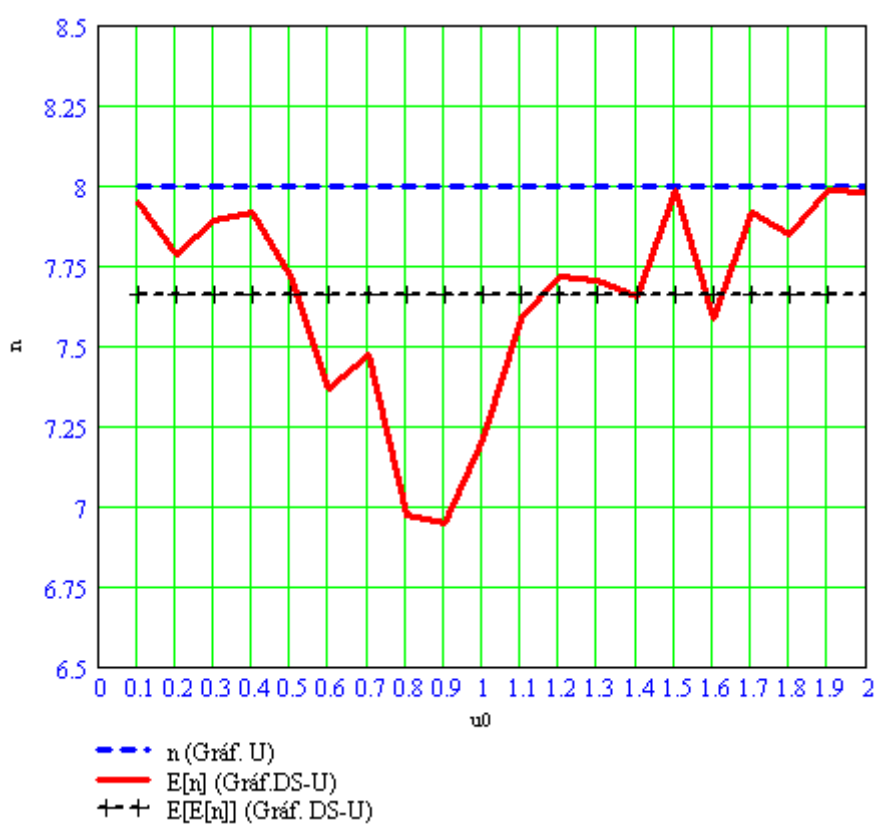

Fig. A.3: Tamaño medio de muestra en el gráfico $D S-U$ para $n=8, u_{0}=1$ y $\alpha_{t}=0.01$ 
APÉNDICE A. COMPARACIÓN DE LA POTENCIA DEL GRÁFICO U FRENTE A LA POTENCIA DEL GRÁFICO DS-U EN UN VALOR U

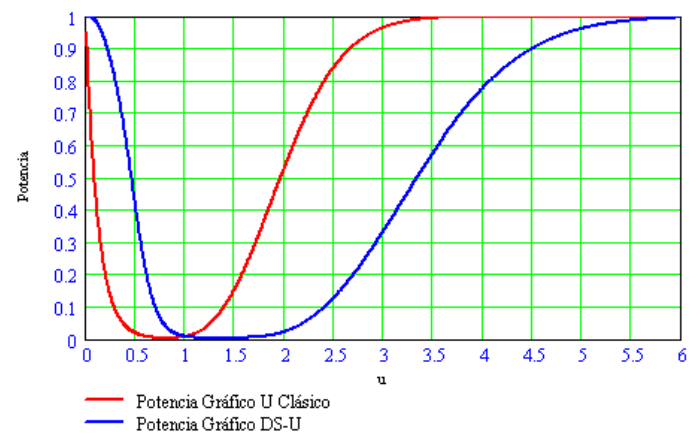

Fig. A.4: Curvas de potencia para $u_{1}=0,1$

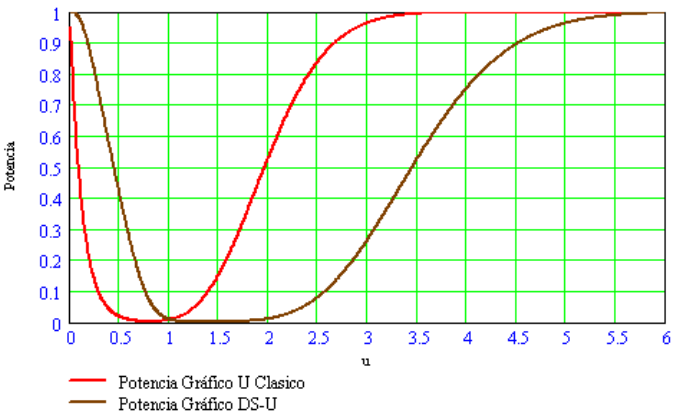

Fig. A.5: Curvas de potencia para $u_{1}=0,2$

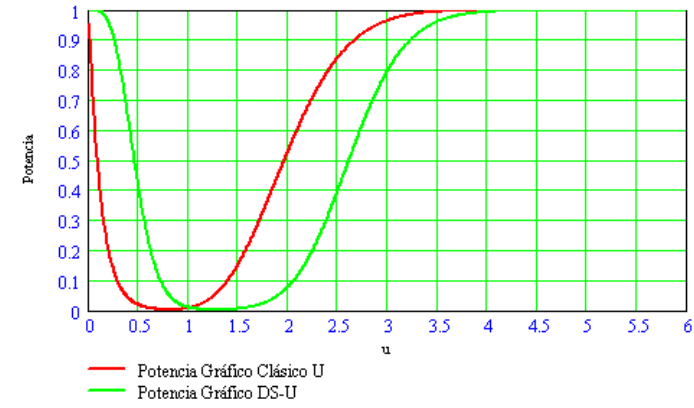

Fig. A.6: Curvas de potencia para $u_{1}=0,3$ 
APÉNDICE A. COMPARACIÓN DE LA POTENCIA DEL GRÁFICO U FRENTE A LA POTENCIA DEL GRÁFICO DS-U EN UN VALOR U

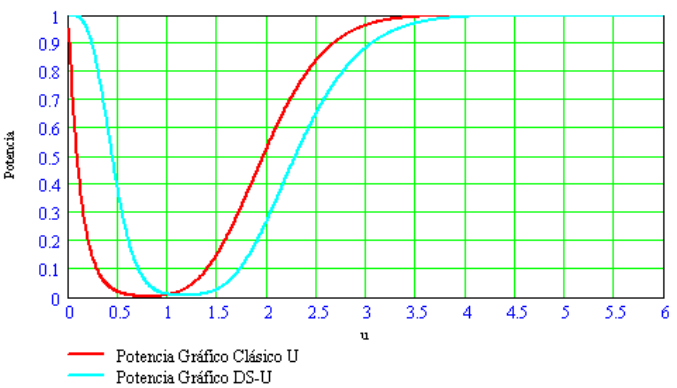

Fig. A.7: Curvas de potencia para $u_{1}=0,4$

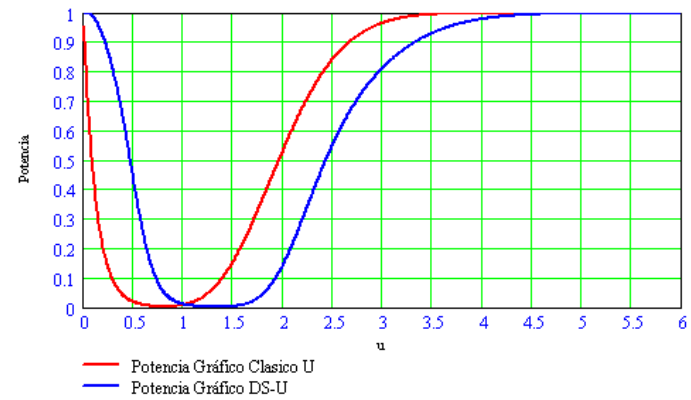

Fig. A.8: Curvas de potencia para $u_{1}=0,5$

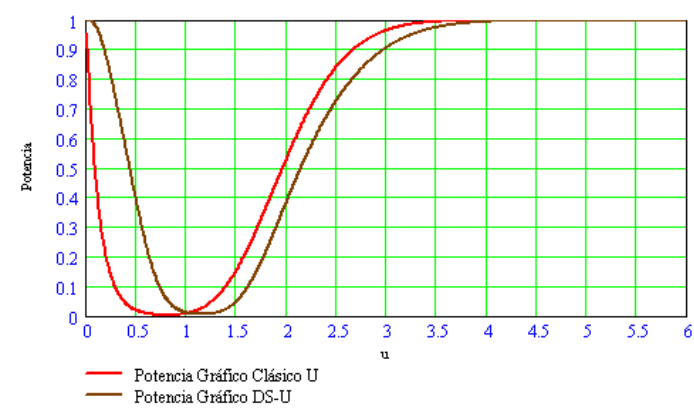

Fig. A.9: Curvas de potencia para $u_{1}=0,6$ 
APÉNDICE A. COMPARACIÓN DE LA POTENCIA DEL GRÁFICO U FRENTE A LA POTENCIA DEL GRÁFICO DS-U EN UN VALOR U

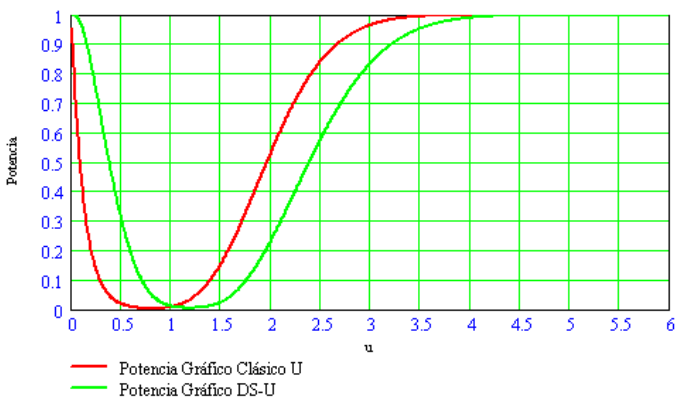

Fig. A.10: Curvas de potencia para $u_{1}=0,7$

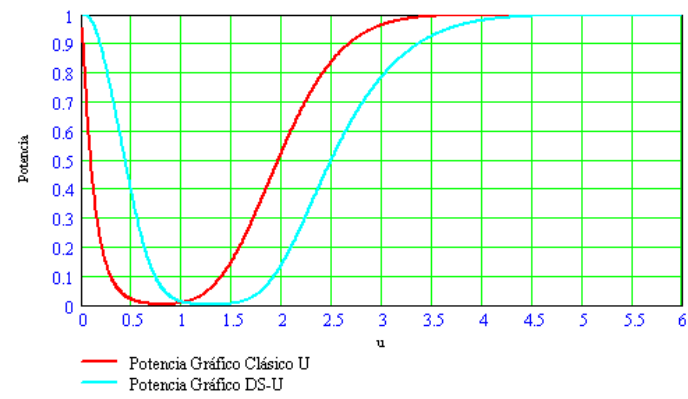

Fig. A.11: Curvas de potencia para $u_{1}=0,8$

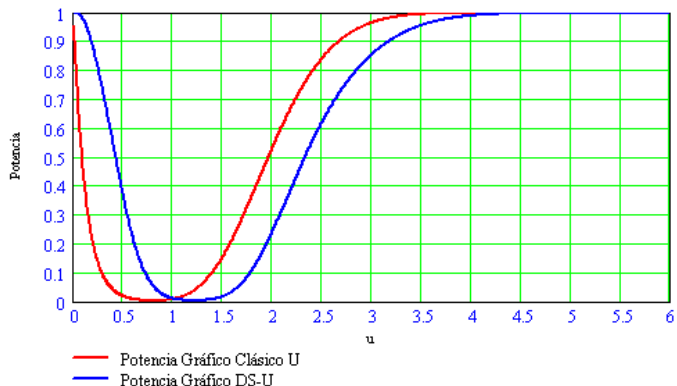

Fig. A.12: Curvas de potencia para $u_{1}=0,9$ 
APÉNDICE A. COMPARACIÓN DE LA POTENCIA DEL GRÁFICO U FRENTE A LA POTENCIA DEL GRÁFICO DS-U EN UN VALOR U

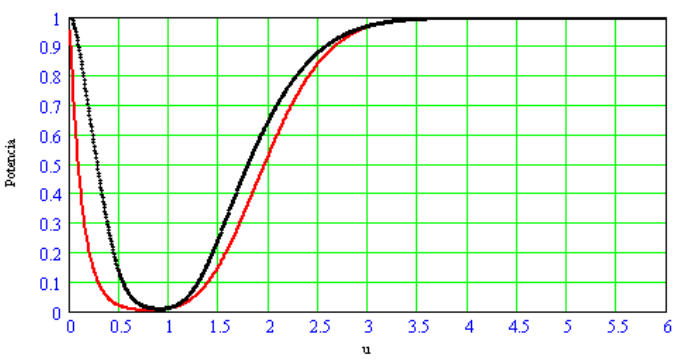

-.. Potencia Gráfico Clásico U

Fig. A.13: Curvas de potencia para $u_{1}=1,0$

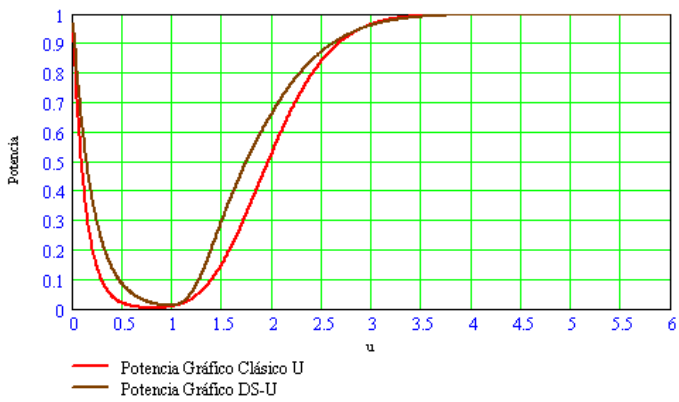

Fig. A.14: Curvas de potencia para $u_{1}=1,1$

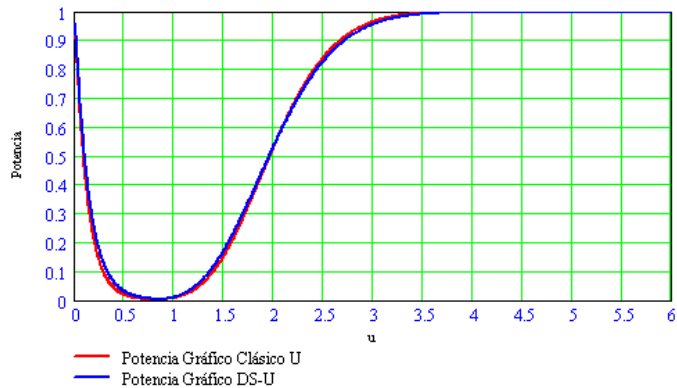

Fig. A.15: Curvas de potencia para $u_{1}=1,2$ 
APÉNDICE A. COMPARACIÓN DE LA POTENCIA DEL GRÁFICO U FRENTE A LA POTENCIA DEL GRÁFICO DS-U EN UN VALOR U

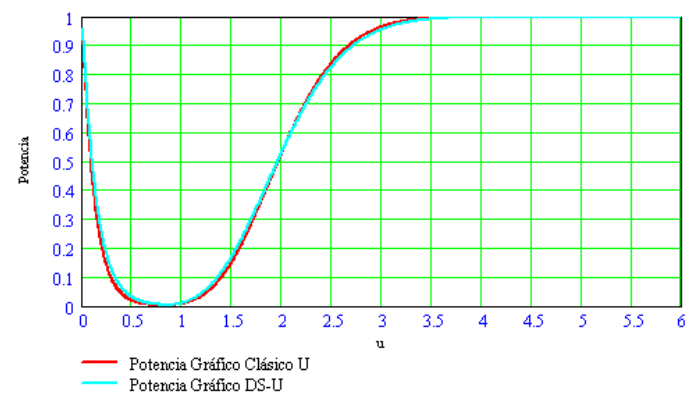

Fig. A.16: Curvas de potencia para $u_{1}=1,3$

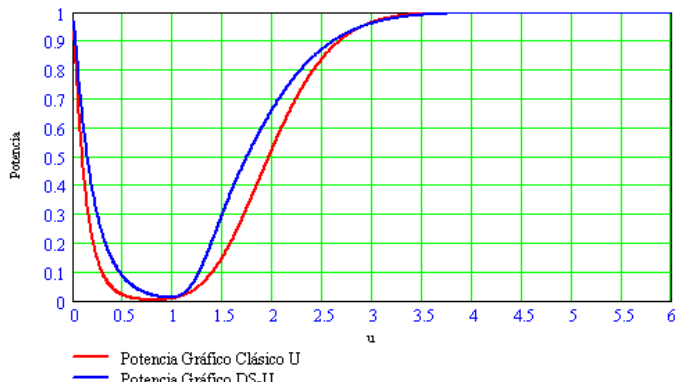

- Potencia Gráfico DS-U

Fig. A.17: Curvas de potencia para $u_{1}=1,4$

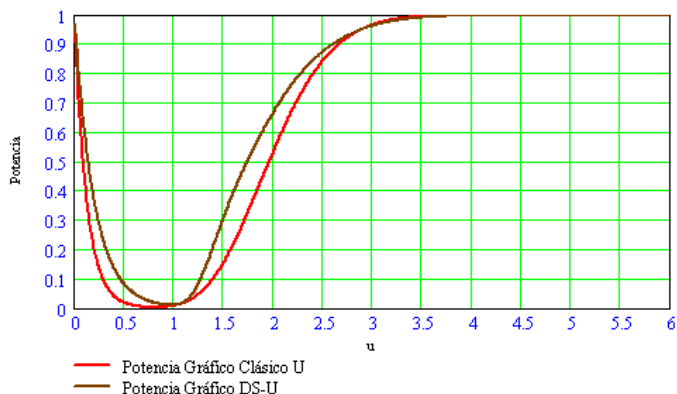

Fig. A.18: Curvas de potencia para $u_{1}=1,5$ 
APÉNDICE A. COMPARACIÓN DE LA POTENCIA DEL GRÁFICO U FRENTE A LA POTENCIA DEL GRÁFICO DS-U EN UN VALOR U

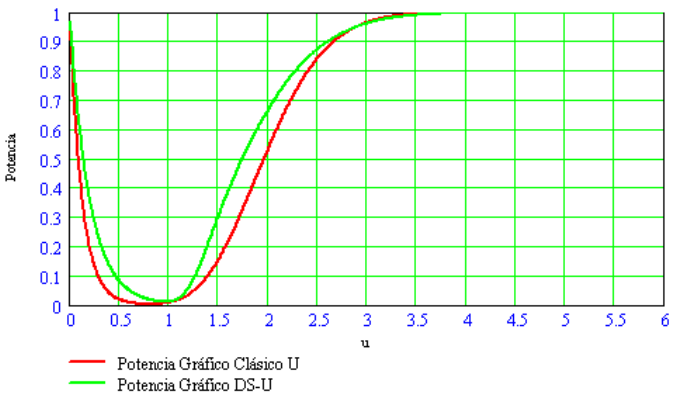

Fig. A.19: Curvas de potencia para $u_{1}=1,6$

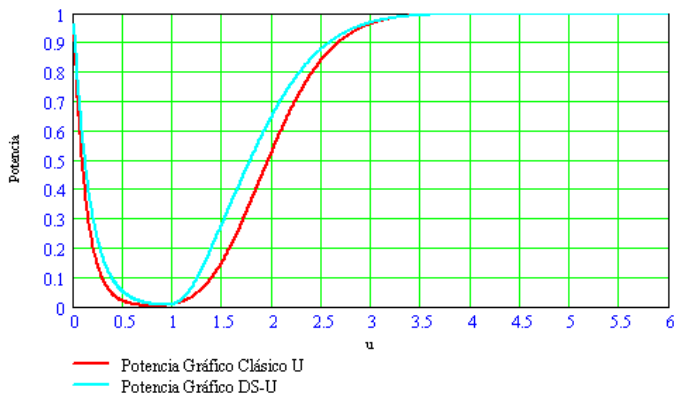

Fig. A.20: Curvas de potencia para $u_{1}=1,7$

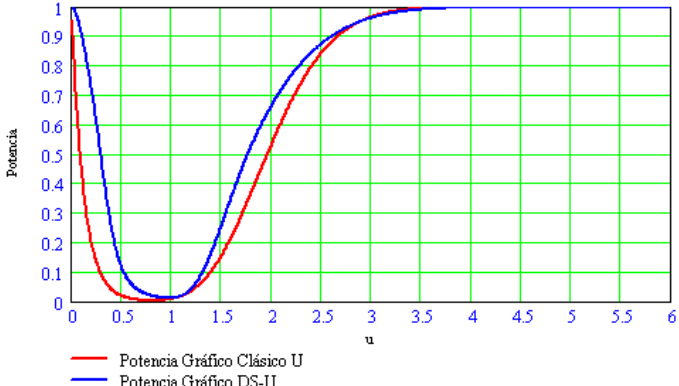

Fig. A.21: Curvas de potencia para $u_{1}=1,8$ 
APÉNDICE A. COMPARACIÓN DE LA POTENCIA DEL GRÁFICO U FRENTE A LA POTENCIA DEL GRÁFICO DS-U EN UN VALOR U

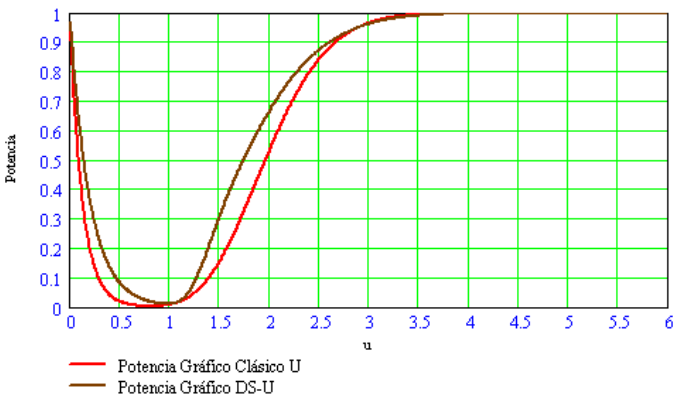

Fig. A.22: Curvas de potencia para $u_{1}=1,9$

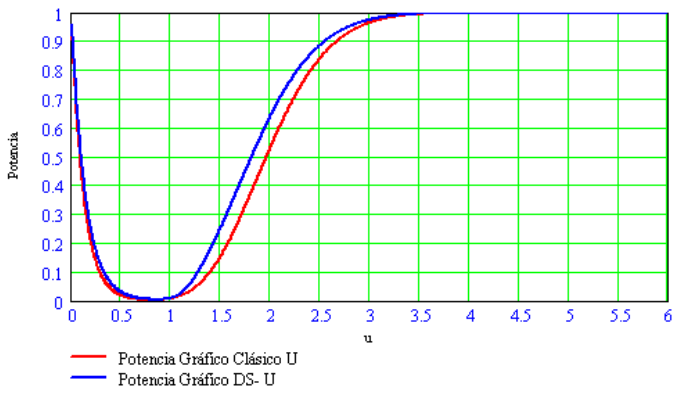

Fig. A.23: Curvas de potencia para $u_{1}=2,0$ 
APÉNDICE A. COMPARACIÓN DE LA POTENCIA DEL GRÁFICO U FRENTE A LA POTENCIA DEL GRÁFICO DS-U EN UN VALOR U

\begin{tabular}{|c||c|c|c|c|c|c||c|c|}
\hline$u_{1}$ & $L C I$ & $L A I$ & $L C I_{1}$ & $L A S$ & $L C S$ & $L C S_{1}$ & $n_{1}$ & $n_{2}$ \\
\hline 0.1 & 0.9 & 2.6 & 15.0 & 16.8 & 48.0 & 44.2 & 5 & 25 \\
0.2 & 0.8 & 2.3 & 23.1 & 20.9 & 43.0 & 45.1 & 6 & 30 \\
0.3 & 0.8 & 3.9 & 6.5 & 12.6 & 17.9 & 32.2 & 6 & 12 \\
0.4 & 0.7 & 3.6 & 6.1 & 15.2 & 45.9 & 17.5 & 7 & 11 \\
0.5 & 0.9 & 2.6 & 13.2 & 11.8 & 18.1 & 44.7 & 5 & 22 \\
0.6 & 0.5 & 2.1 & 14.8 & 12.1 & 50.3 & 31.8 & 6 & 20 \\
0.7 & 0.7 & 2.7 & 13.3 & 16.4 & 19.2 & 23.2 & 7 & 16 \\
0.8 & 0.6 & 2.5 & 11.5 & 14.5 & 19.5 & 30.6 & 6 & 16 \\
0.9 & 0.9 & 2.8 & 10.5 & 13.7 & 18.7 & 26.8 & 6 & 15 \\
\hline 1.0 & 0.6 & 1.4 & 10.4 & 10.7 & 19.0 & 25.1 & 6 & 21 \\
\hline 1.1 & 0.8 & 0.9 & 15.0 & 8.2 & 15.5 & 47.3 & 5 & 38 \\
1.2 & 0.7 & 0.8 & 4.0 & 13.8 & 19.3 & 50.5 & 7 & 56 \\
1.3 & 0.2 & 0.6 & 1.8 & 13.6 & 19.8 & 49.7 & 7 & 55 \\
1.4 & 0.4 & 0.9 & 13.8 & 8.8 & 16.5 & 48.8 & 5 & 39 \\
1.5 & 0.5 & 0.9 & 5.2 & 8.4 & 14.2 & 55.9 & 5 & 44 \\
1.6 & 0.3 & 0.8 & 5.4 & 8.6 & 15.3 & 47.4 & 5 & 38 \\
1.7 & 0.4 & 0.8 & 18.6 & 10.2 & 27.7 & 51.9 & 6 & 45 \\
1.8 & 0.2 & 1.6 & 10.3 & 8.6 & 16.3 & 37.1 & 5 & 28 \\
1.9 & 0.6 & 0.8 & 5.0 & 8.8 & 14.9 & 55.3 & 5 & 44 \\
2.0 & 0.5 & 0.9 & 14.8 & 12.7 & 14.0 & 52.3 & 7 & 46 \\
\hline
\end{tabular}

Tabla A.1: Parámetros obtenidos en la comparación de la potencia para el nuevo gráfico $D S-U: u_{0}=1, \alpha_{t}=0.01, n=8$. 
APÉNDICE A. COMPARACIÓN DE LA POTENCIA DEL GRÁFICO U FRENTE A LA POTENCIA DEL GRÁFICO DS-U EN UN VALOR U

\begin{tabular}{|c||c|c||c|c|}
\hline$u_{1}$ & Pot $_{u}$ & Pot $_{D S-U}$ & Pot $_{D S-U}-$ Pot $_{U}$ & $\alpha_{\text {real }}$ \\
\hline 0.1 & 0.449329 & 0.985612 & 0.536283 & $8.224174 \cdot 10^{-3}$ \\
0.2 & 0.201897 & 0.879487 & 0.677591 & $9.534652 \cdot 10^{-3}$ \\
0.3 & 0.090718 & 0.838096 & 0.747378 & $9.752556 \cdot 10^{-3}$ \\
0.4 & 0.040762 & 0.593258 & 0.552496 & 0.010138 \\
0.5 & 0.018321 & 0.442839 & 0.424518 & 0.010168 \\
0.6 & 0.008272930 & 0.240035 & 0.231762 & 0.010196 \\
0.7 & 0.003941482 & 0.103513 & 0.099572 & 0.010180 \\
0.8 & 0.002653148 & 0.058487 & 0.055834 & 0.010250 \\
0.9 & 0.003895493 & 0.023954 & 0.020059 & 0.010181 \\
1.0 & 0.008566474 & 0.012174 & 0.003607101 & 0.012174 \\
1.1 & 0.018553 & 0.024035 & 0.005482492 & 0.011274 \\
1.2 & 0.036270 & 0.046969 & 0.010699 & 0.010732 \\
1.3 & 0.064081 & 0.078870 & 0.014788 & 0.010759 \\
1.4 & 0.103676 & 0.216075 & 0.112398 & 0.011388 \\
1.5 & 0.155590 & 0.307309 & 0.151718 & 0.010056 \\
1.6 & 0.218981 & 0.391866 & 0.172885 & 0.011274 \\
1.7 & 0.291712 & 0.441444 & 0.149732 & $9.541468 \cdot 10^{-3}$ \\
1.8 & 0.370699 & 0.529507 & 0.158807 & 0.010968 \\
1.9 & 0.452389 & 0.607941 & 0.155553 & 0.010056 \\
2.0 & 0.533255 & 0.641542 & 0.108286 & 0.010210 \\
\hline & & & & \\
\hline
\end{tabular}

Tabla A.2: Resultados para $u_{0}=1, \alpha_{\text {teórico }}=0.01$ y $n=8$ 
APÉNDICE A. COMPARACIÓN DE LA POTENCIA DEL GRÁFICO U FRENTE A LA POTENCIA DEL GRÁFICO DS-U EN UN VALOR U

A.2. Optimización del gráfico de control u con

$$
n=8, u_{0}=1 \mathbf{y} \alpha_{t}=0.05
$$


APÉNDICE A. COMPARACIÓN DE LA POTENCIA DEL GRÁFICO U FRENTE A LA POTENCIA DEL GRÁFICO DS-U EN UN VALOR U

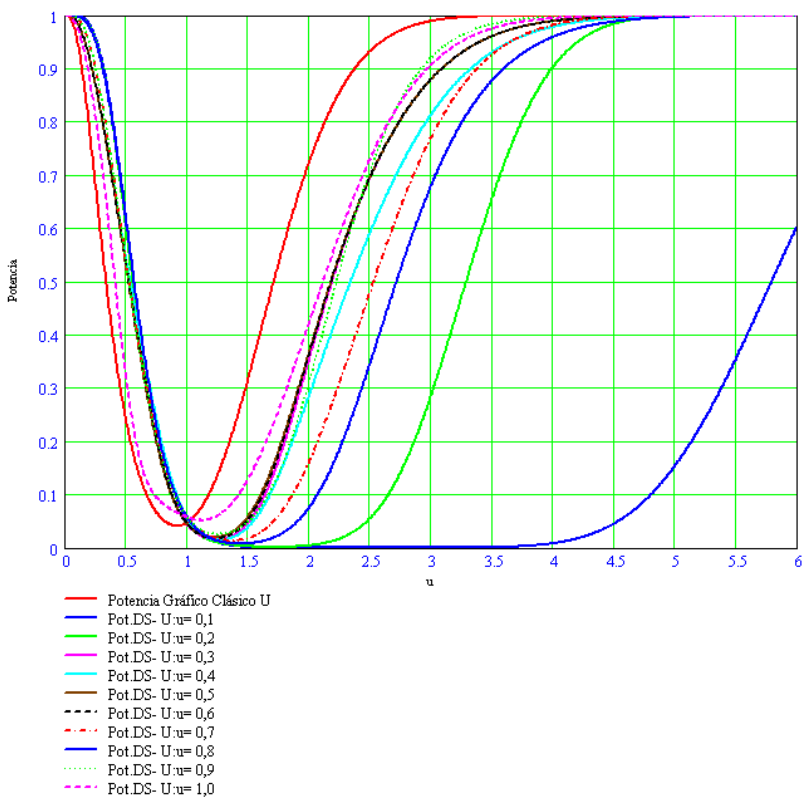

(a) $u_{1} \in[0.1,1.0]$

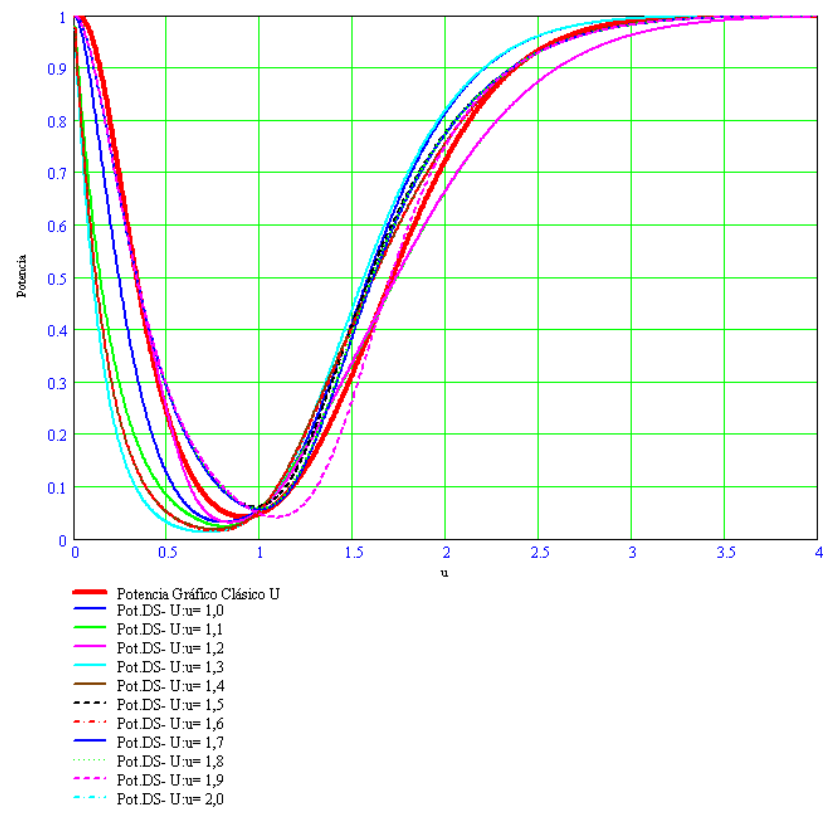

(b) $u_{1} \in[1.1,2.0]$

Fig. A.24: Curvas de potencia en distintos valores de $u_{1}$ 
APÉNDICE A. COMPARACIÓN DE LA POTENCIA DEL GRÁFICO U FRENTE A LA POTENCIA DEL GRÁFICO DS-U EN UN VALOR U

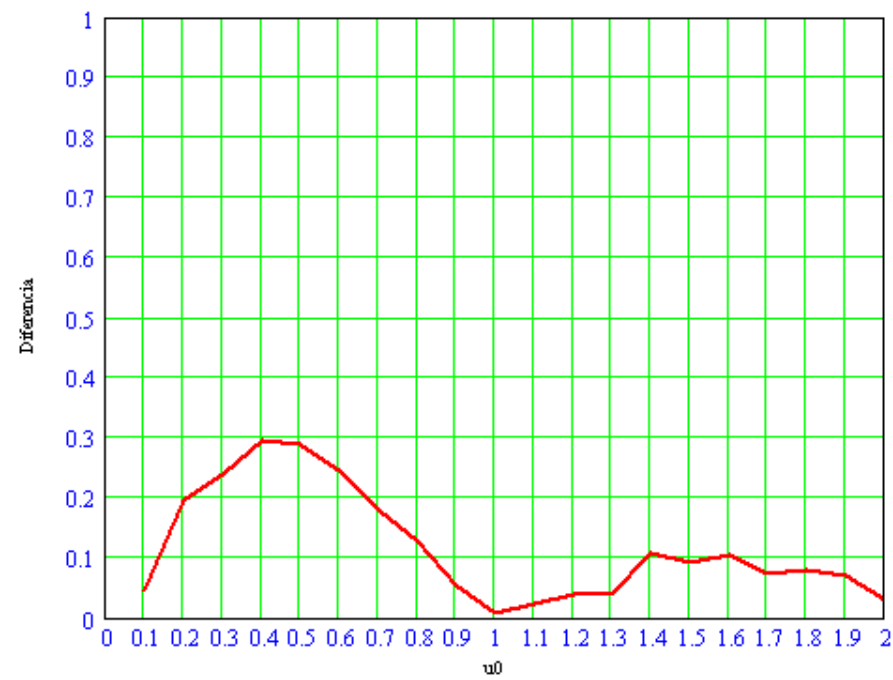

Fig. A.25: $\operatorname{Pot}_{D S-U}-\operatorname{Pot}_{u}$ para $n=8, u_{0}=1$ y $\alpha_{t}=0.05$

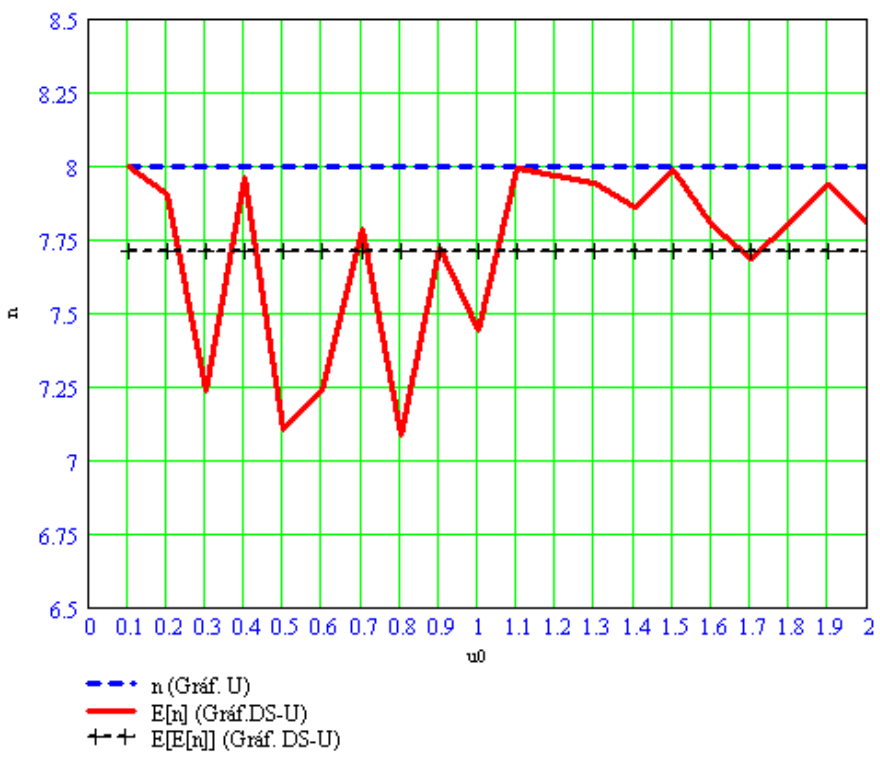

Fig. A.26: Tamaño medio de muestra en el gráfico $D S-U$ para $n=8, u_{0}=1$ y $\alpha_{t}=0.05$ 
APÉNDICE A. COMPARACIÓN DE LA POTENCIA DEL GRÁFICO U FRENTE A LA POTENCIA DEL GRÁFICO DS-U EN UN VALOR U

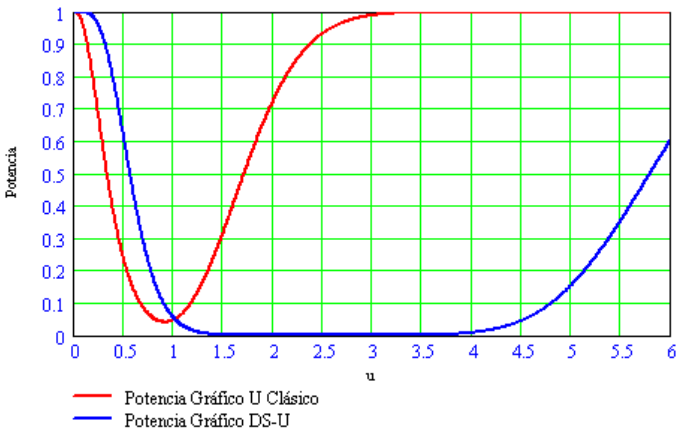

Fig. A.27: Curvas de potencia para $u_{1}=0,1$

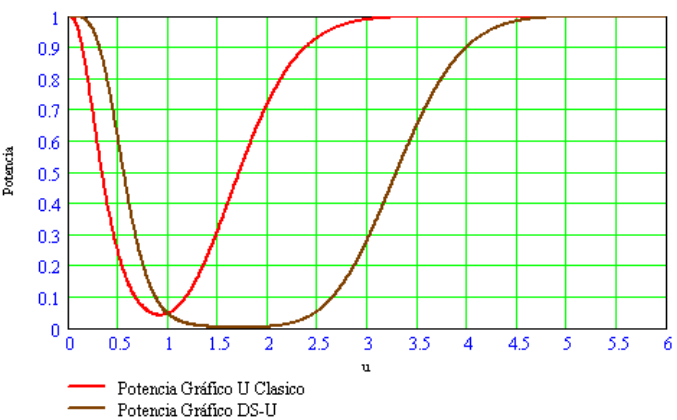

Fig. A.28: Curvas de potencia para $u_{1}=0,2$

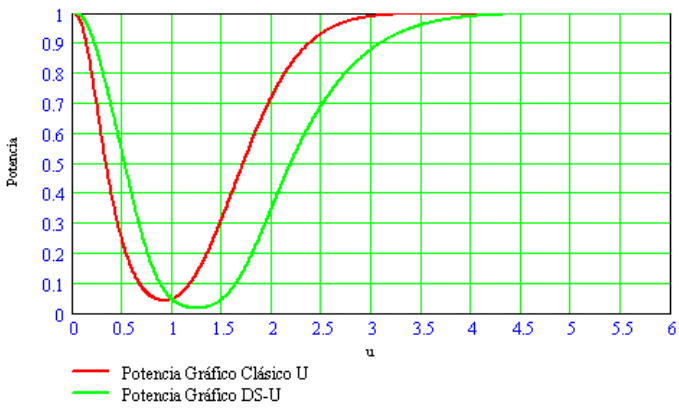

Fig. A.29: Curvas de potencia para $u_{1}=0,3$ 
APÉNDICE A. COMPARACIÓN DE LA POTENCIA DEL GRÁFICO U FRENTE A LA POTENCIA DEL GRÁFICO DS-U EN UN VALOR U

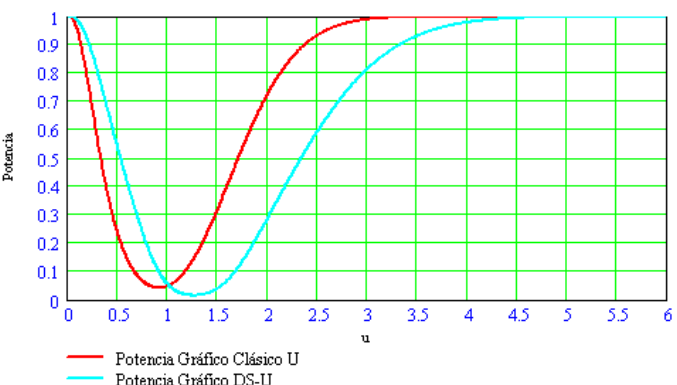

Fig. A.30: Curvas de potencia para $u_{1}=0,4$

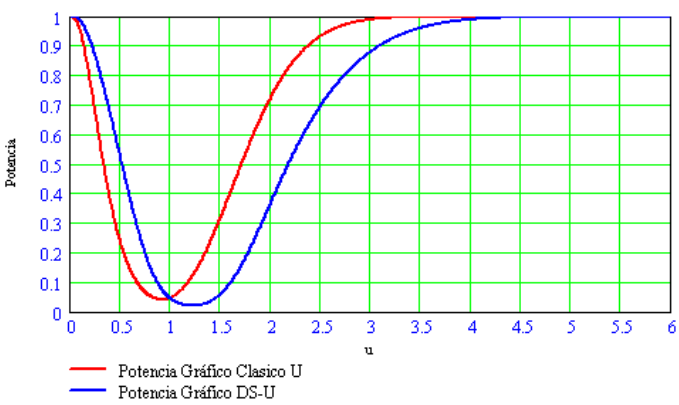

Fig. A.31: Curvas de potencia para $u_{1}=0,5$

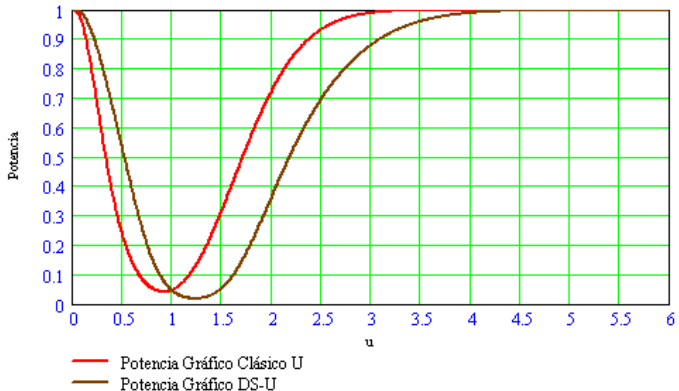

Fig. A.32: Curvas de potencia para $u_{1}=0,6$ 
APÉNDICE A. COMPARACIÓN DE LA POTENCIA DEL GRÁFICO U FRENTE A LA POTENCIA DEL GRÁFICO DS-U EN UN VALOR U

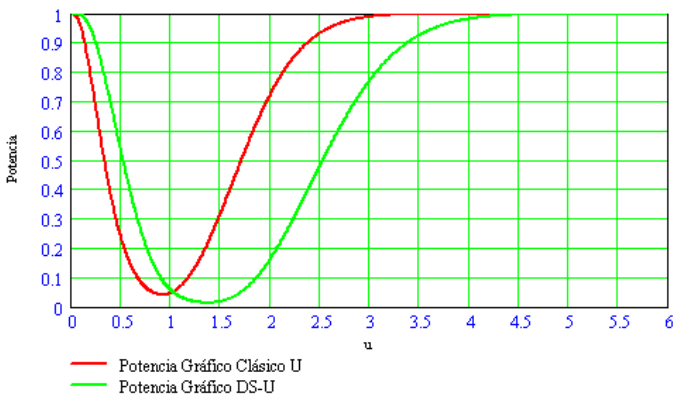

Fig. A.33: Curvas de potencia para $u_{1}=0,7$

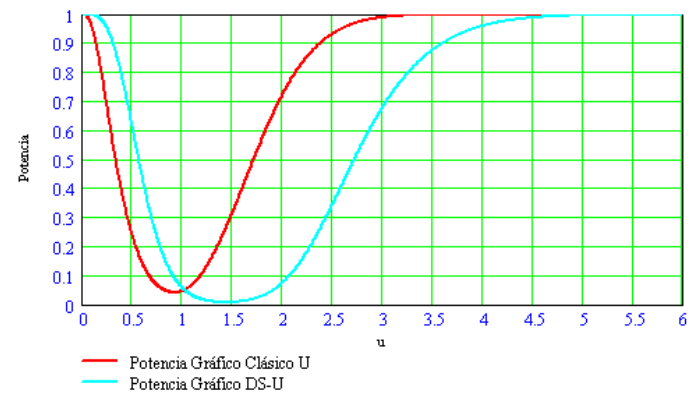

Fig. A.34: Curvas de potencia para $u_{1}=0,8$

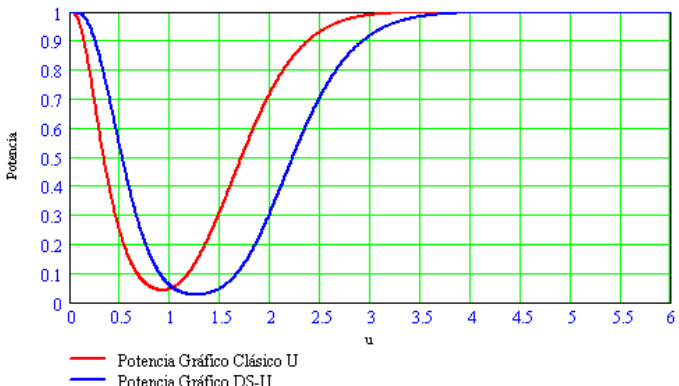

Fig. A.35: Curvas de potencia para $u_{1}=0,9$ 
APÉNDICE A. COMPARACIÓN DE LA POTENCIA DEL GRÁFICO U FRENTE A LA POTENCIA DEL GRÁFICO DS-U EN UN VALOR U

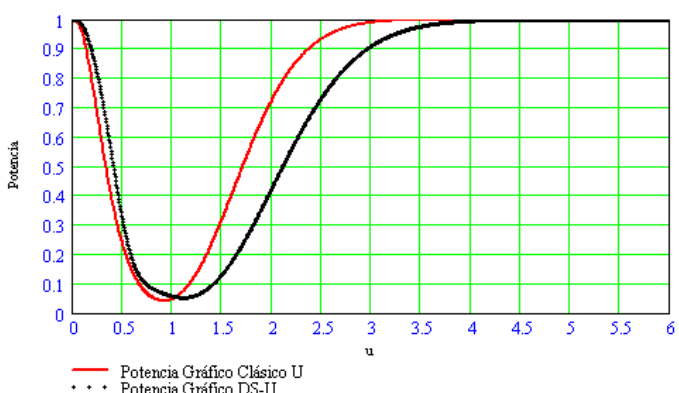

.. Potencia Gráfico Clásico U

Fig. A.36: Curvas de potencia para $u_{1}=1,0$

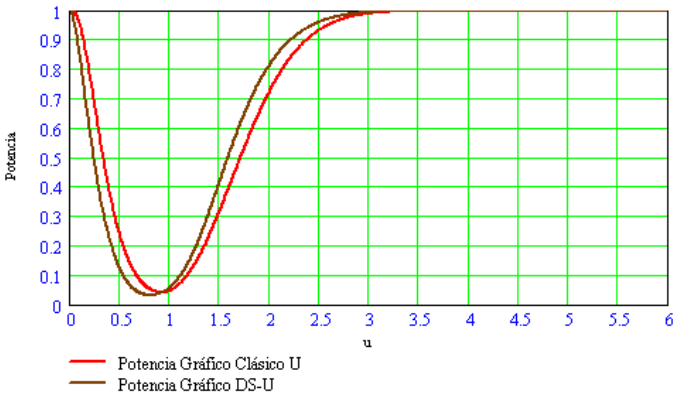

Fig. A.37: Curvas de potencia para $u_{1}=1,1$

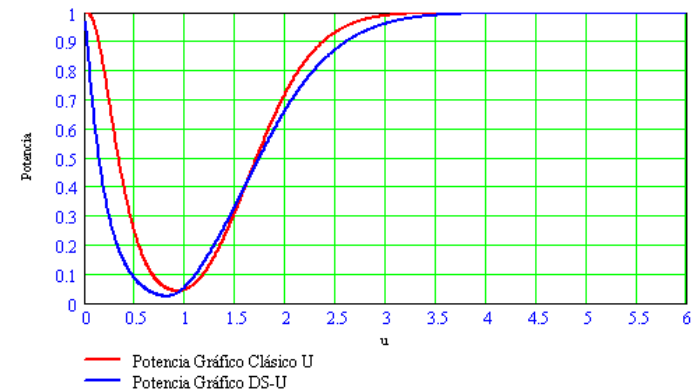

Fig. A.38: Curvas de potencia para $u_{1}=1,2$ 
APÉNDICE A. COMPARACIÓN DE LA POTENCIA DEL GRÁFICO U FRENTE A LA POTENCIA DEL GRÁFICO DS-U EN UN VALOR U

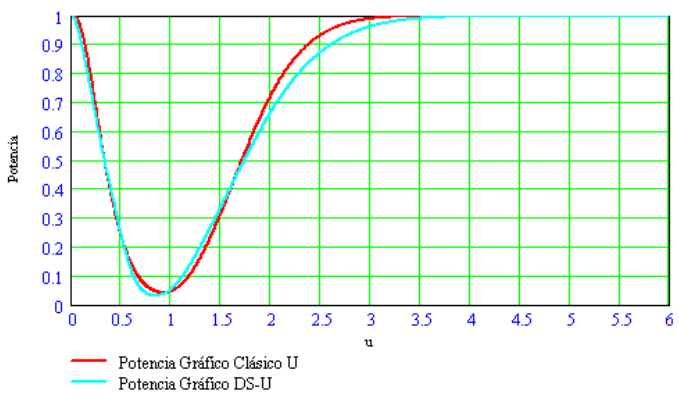

Fig. A.39: Curvas de potencia para $u_{1}=1,3$

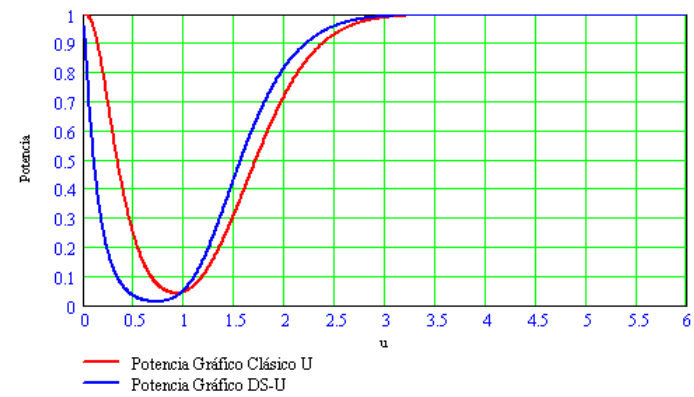

Fig. A.40: Curvas de potencia para $u_{1}=1,4$

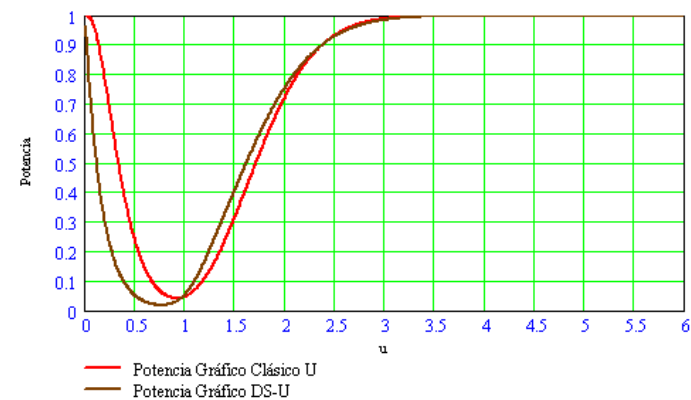

Fig. A.41: Curvas de potencia para $u_{1}=1,5$ 
APÉNDICE A. COMPARACIÓN DE LA POTENCIA DEL GRÁFICO U FRENTE A LA POTENCIA DEL GRÁFICO DS-U EN UN VALOR U

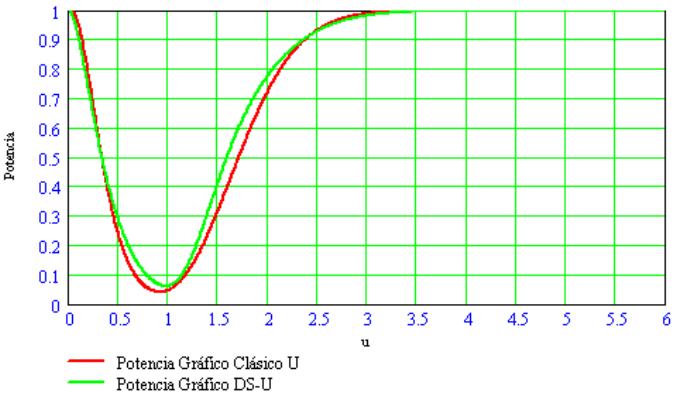

Fig. A.42: Curvas de potencia para $u_{1}=1,6$

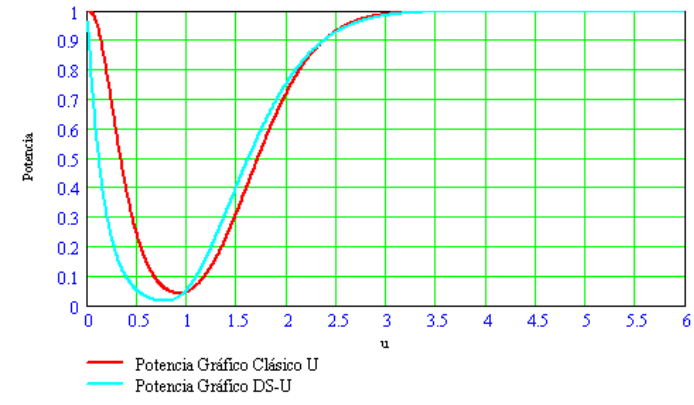

Fig. A.43: Curvas de potencia para $u_{1}=1,7$

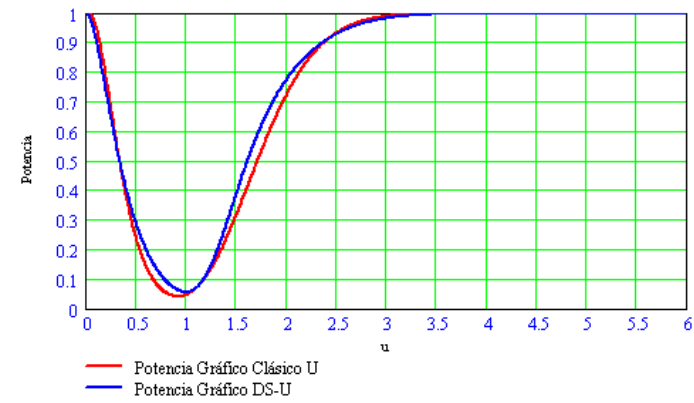

Fig. A.44: Curvas de potencia para $u_{1}=1,8$ 
APÉNDICE A. COMPARACIÓN DE LA POTENCIA DEL GRÁFICO U FRENTE A LA POTENCIA DEL GRÁFICO DS-U EN UN VALOR U

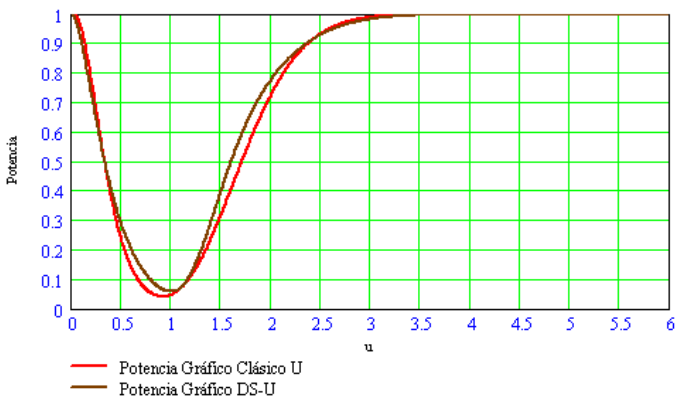

Fig. A.45: Curvas de potencia para $u_{1}=1,9$

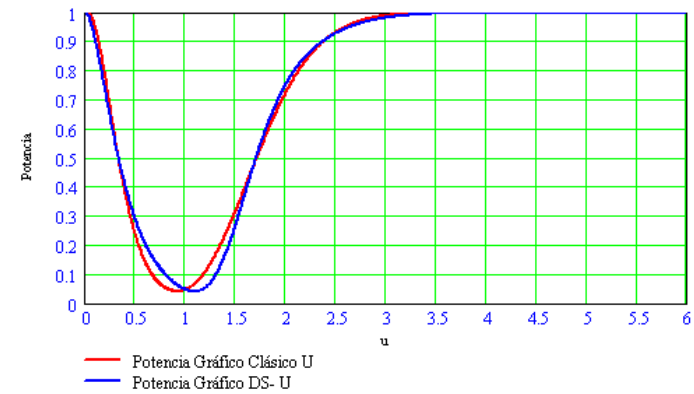

Fig. A.46: Curvas de potencia para $u_{1}=2,0$ 
APÉNDICE A. COMPARACIÓN DE LA POTENCIA DEL GRÁFICO U FRENTE A LA POTENCIA DEL GRÁFICO DS-U EN UN VALOR U

\begin{tabular}{|c||c|c|c|c|c|c||c|c|}
\hline$u_{1}$ & $L C I$ & $L A I$ & $L C I_{1}$ & $L A S$ & $L C S$ & $L C S_{1}$ & $n_{1}$ & $n_{2}$ \\
\hline 0.1 & 1.3 & 4.4 & 4.9 & 21.8 & 41.5 & 41.7 & 7 & 6 \\
0.2 & 0.9 & 3.1 & 6.9 & 9.6 & 19.3 & 34.2 & 5 & 10 \\
0.3 & 0.5 & 2.9 & 15 & 10.5 & 15.8 & 28.1 & 5 & 17 \\
0.4 & 0.7 & 2.5 & 22.5 & 11.3 & 27.8 & 37.8 & 5 & 24 \\
0.5 & 0.3 & 2.9 & 14 & 10.4 & 45 & 25.5 & 5 & 16 \\
0.6 & 0.8 & 2.8 & 15 & 10.8 & 35 & 27.3 & 5 & 17 \\
0.7 & 2.1 & 3.4 & 14.7 & 17.7 & 43.3 & 21.5 & 7 & 15 \\
0.8 & 0.6 & 3.4 & 5.5 & 12.7 & 16.1 & 18.2 & 5 & 8 \\
0.9 & 1.8 & 3.8 & 9.8 & 14.9 & 25.3 & 15.5 & 7 & 9 \\
\hline 1.0 & 1.8 & 2.5 & 14 & 12.3 & 18.6 & 23.4 & 6 & 27 \\
\hline 1.1 & 0.5 & 1.7 & 7.4 & 10.9 & 14.9 & 11.7 & 7 & 10 \\
1.2 & 0.3 & 0.7 & 12.5 & 8.2 & 13.9 & 39.3 & 5 & 44 \\
1.3 & 0.6 & 1.4 & 24.1 & 8.7 & 9.2 & 46.5 & 5 & 42 \\
1.4 & 0.2 & 0.5 & 5.2 & 10.3 & 13.9 & 10.6 & 7 & 10 \\
1.5 & 0.5 & 0.8 & 19.6 & 9.6 & 10.7 & 52.8 & 6 & 48 \\
1.6 & 1.3 & 1.8 & 7.9 & 8.0 & 14.2 & 25.3 & 5 & 21 \\
1.7 & 0.4 & 0.5 & 8.6 & 9.1 & 13.2 & 19.2 & 6 & 21 \\
1.8 & 1.1 & 1.2 & 1.4 & 7.1 & 23.5 & 26.4 & 5 & 21 \\
1.9 & 1.1 & 1.7 & 11.7 & 7.7 & 43 & 27.2 & 5 & 22 \\
2.0 & 1.3 & 1.6 & 12.9 & 7.7 & 25.4 & 30.1 & 5 & 21 \\
\hline
\end{tabular}

Tabla A.3: Parámetros obtenidos en la comparación de la potencia para el nuevo gráfico $D S-U: u_{0}=1, \alpha_{t}=0.05, n=8$. 
APÉNDICE A. COMPARACIÓN DE LA POTENCIA DEL GRÁFICO U FRENTE A LA POTENCIA DEL GRÁFICO DS-U EN UN VALOR U

\begin{tabular}{|c||c|c||c|c|}
\hline$u_{1}$ & Pot $_{u}$ & $P_{D S-U}$ & Pot $_{D S-U}-$ Pot $_{U}$ & $\alpha_{\text {real }}$ \\
\hline 0.1 & 0.952577 & 0.999153 & 0.046576 & 0.054529 \\
0.2 & 0.783358 & 0.978232 & 0.194874 & 0.044494 \\
0.3 & 0.569709 & 0.808685 & 0.238976 & 0.044403 \\
0.4 & 0.379911 & 0.676587 & 0.296676 & 0.055678 \\
0.5 & 0.238180 & 0.528091 & 0.289911 & 0.044593 \\
0.6 & 0.143012 & 0.388247 & 0.245235 & 0.044861 \\
0.7 & 0.084370 & 0.263166 & 0.178797 & 0.056866 \\
0.8 & 0.052575 & 0.177751 & 0.125176 & 0.056703 \\
0.9 & 0.041375 & 0.095569 & 0.054194 & 0.056165 \\
\hline 1.0 & 0.047935 & 0.057243 & 0.009308458 & 0.057243 \\
\hline 1.1 & 0.071534 & 0.095654 & 0.024120 & 0.058548 \\
1.2 & 0.111987 & 0.150885 & 0.038898 & 0.057775 \\
1.3 & 0.168405 & 0.206775 & 0.038370 & 0.055451 \\
1.4 & 0.238580 & 0.345976 & 0.107395 & 0.055211 \\
1.5 & 0.318987 & 0.411730 & 0.092743 & 0.055570 \\
1.6 & 0.405243 & 0.509595 & 0.104352 & 0.062316 \\
1.7 & 0.492756 & 0.566179 & 0.073424 & 0.055637 \\
1.8 & 0.577342 & 0.658555 & 0.081213 & 0.056090 \\
1.9 & 0.655677 & 0.724931 & 0.069254 & 0.057784 \\
2.0 & 0.725505 & 0.754594 & 0.029088 & 0.046923 \\
\hline
\end{tabular}

Tabla A.4: Resultados para $u_{0}=1, \alpha_{\text {teórico }}=0.05$ y $n=8$ 
APÉNDICE A. COMPARACIÓN DE LA POTENCIA DEL GRÁFICO U FRENTE A LA POTENCIA DEL GRÁFICO DS-U EN UN VALOR U

A.3. Comparación de los gráficos de control $u$ y $\boldsymbol{D S}-\boldsymbol{U} \operatorname{con} n=8, u_{0}=1$ y $\alpha_{t}=0.0027$ 
APÉNDICE A. COMPARACIÓN DE LA POTENCIA DEL GRÁFICO U FRENTE A LA POTENCIA DEL GRÁFICO DS-U EN UN VALOR U

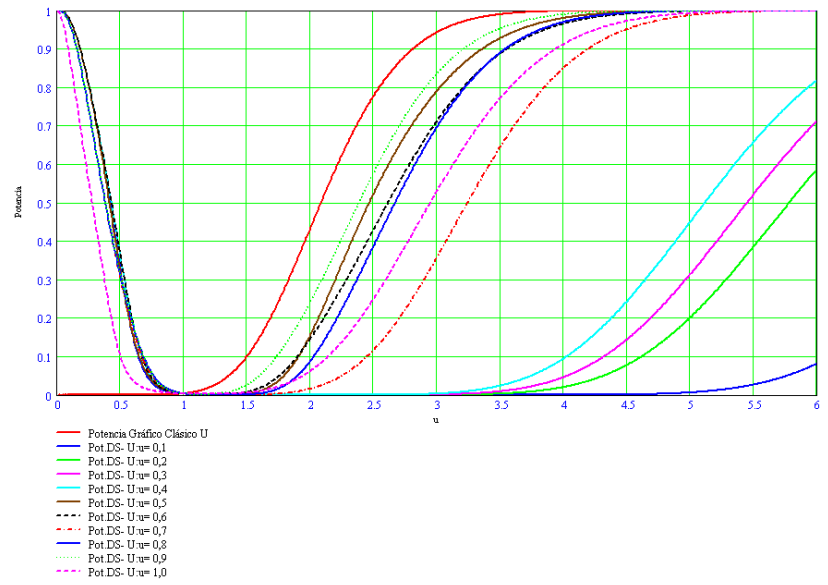

(a) $u_{1} \in[0.1,1.0]$

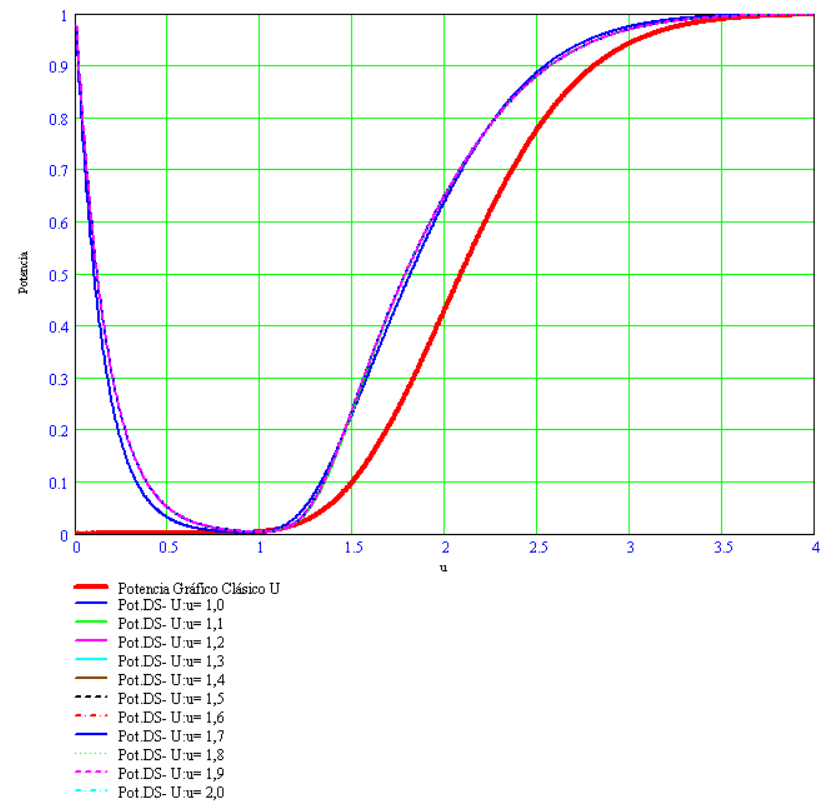

(b) $u_{1} \in[1.1,2.0]$

Fig. A.47: Curvas de potencia en distintos valores de $u_{1}$ 
APÉNDICE A. COMPARACIÓN DE LA POTENCIA DEL GRÁFICO U FRENTE A LA POTENCIA DEL GRÁFICO DS-U EN UN VALOR U

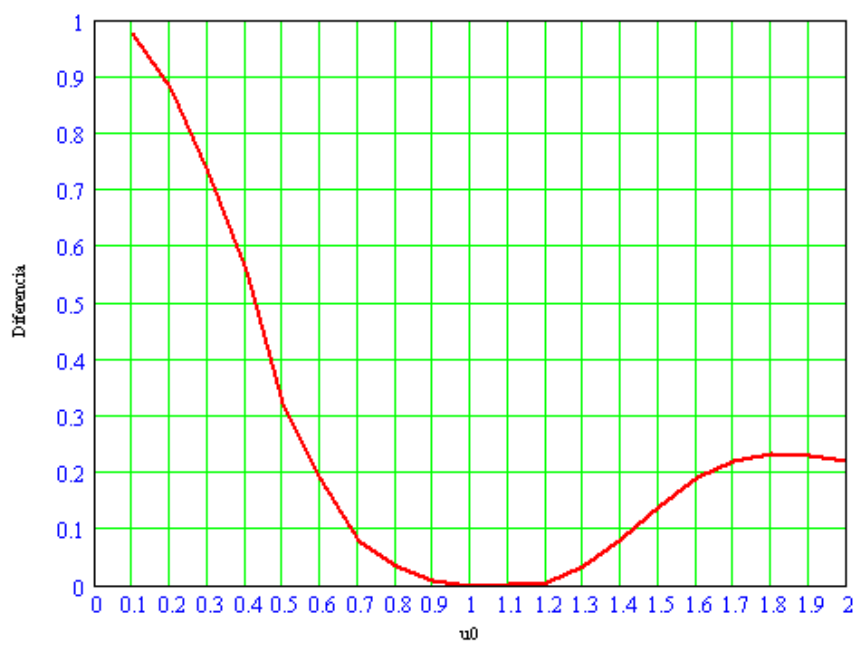

Fig. A.48: $\operatorname{Pot}_{D S-U}-$ Pot $_{u}$ para $n=8, u_{0}=1$ y $\alpha_{t}=0.0027$

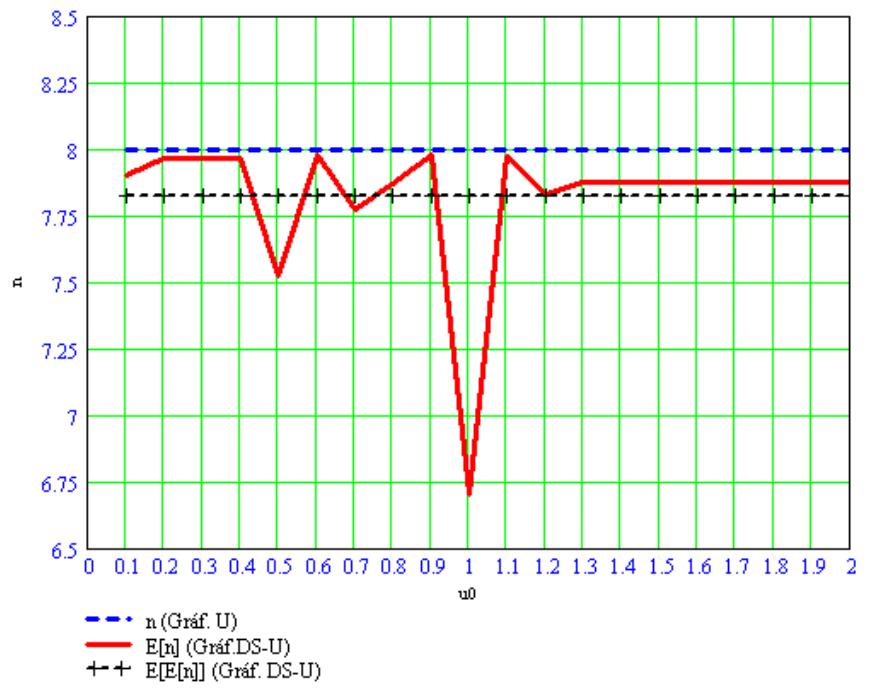

Fig. A.49: Tamaño medio de muestra en el gráfico $D S-U$ para $n=8, u_{0}=1$ y $\alpha_{t}=0.0027$ 
APÉNDICE A. COMPARACIÓN DE LA POTENCIA DEL GRÁFICO U FRENTE A LA POTENCIA DEL GRÁFICO DS-U EN UN VALOR U

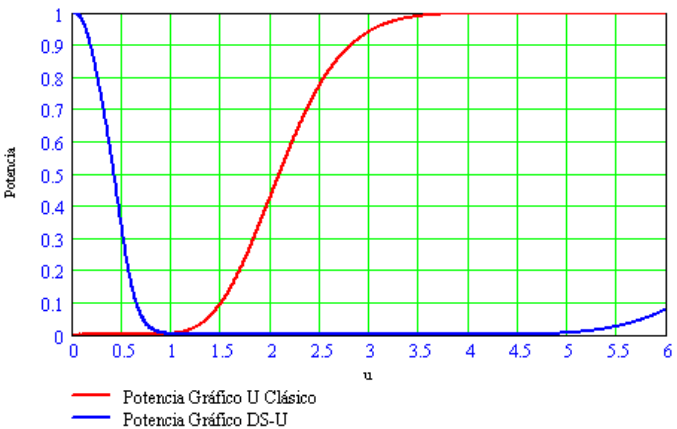

Fig. A.50: Curvas de potencia para $u_{1}=0,1$

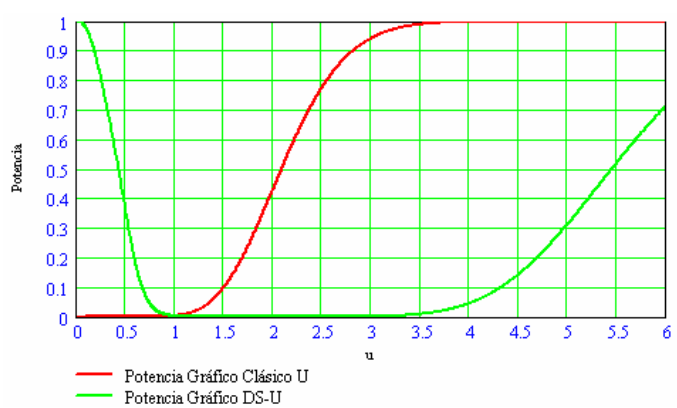

Fig. A.51: Curvas de potencia para $u_{1}=0,2$

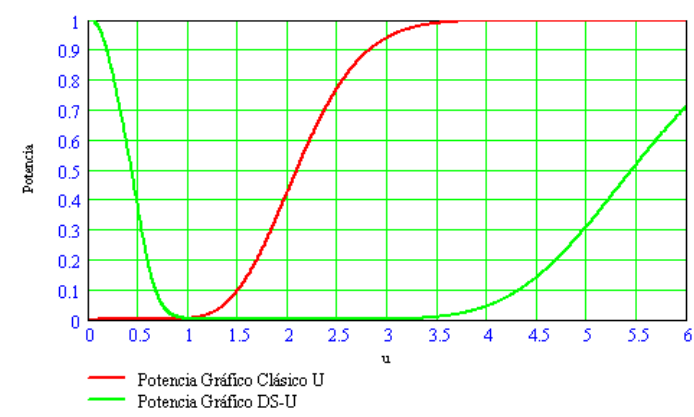

Fig. A.52: Curvas de potencia para $u_{1}=0,3$ 
APÉNDICE A. COMPARACIÓN DE LA POTENCIA DEL GRÁFICO U FRENTE A LA POTENCIA DEL GRÁFICO DS-U EN UN VALOR U

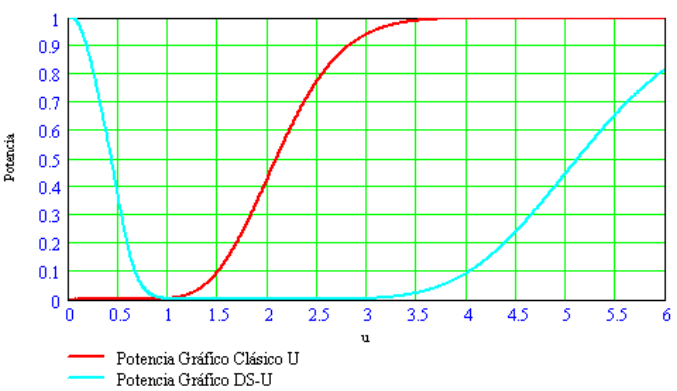

Fig. A.53: Curvas de potencia para $u_{1}=0,4$

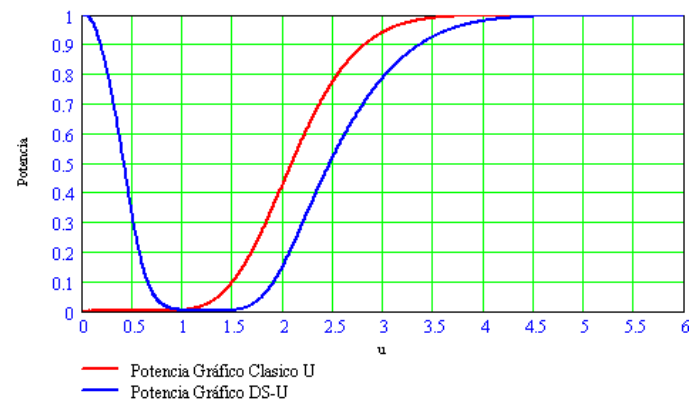

Fig. A.54: Curvas de potencia para $u_{1}=0,5$

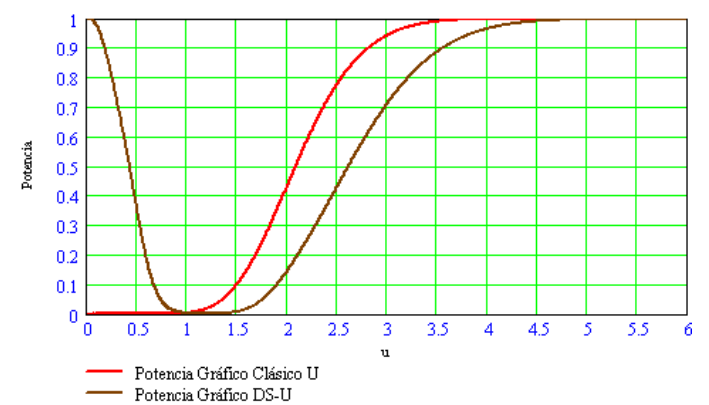

Fig. A.55: Curvas de potencia para $u_{1}=0,6$ 
APÉNDICE A. COMPARACIÓN DE LA POTENCIA DEL GRÁFICO U FRENTE A LA POTENCIA DEL GRÁFICO DS-U EN UN VALOR U

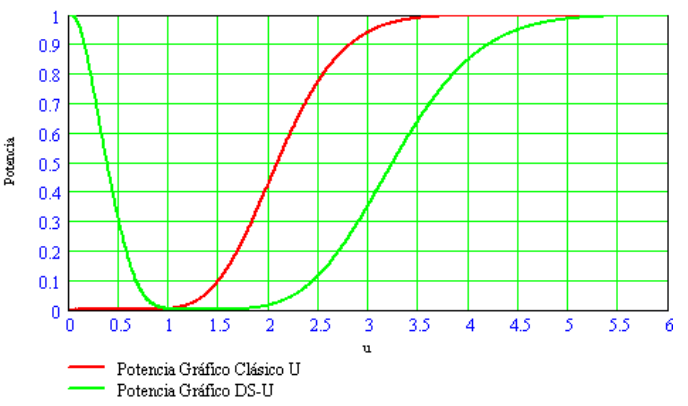

Fig. A.56: Curvas de potencia para $u_{1}=0,7$

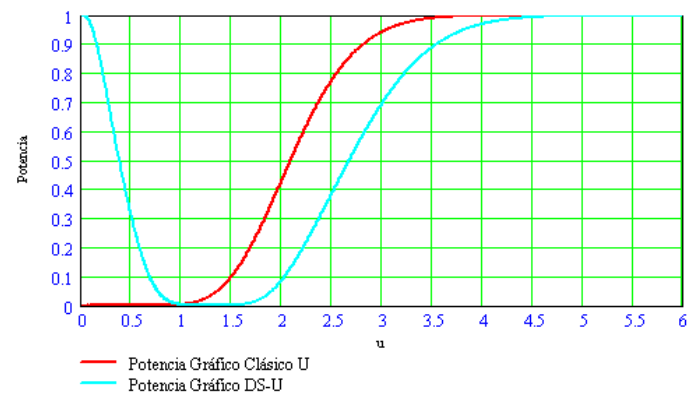

Fig. A.57: Curvas de potencia para $u_{1}=0,8$

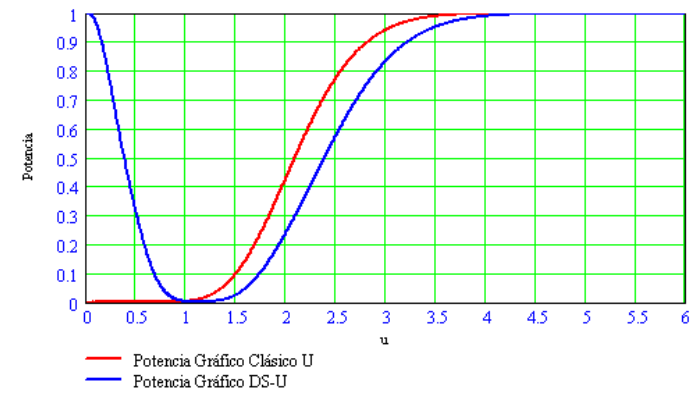

Fig. A.58: Curvas de potencia para $u_{1}=0,9$ 
APÉNDICE A. COMPARACIÓN DE LA POTENCIA DEL GRÁFICO U FRENTE A LA POTENCIA DEL GRÁFICO DS-U EN UN VALOR U

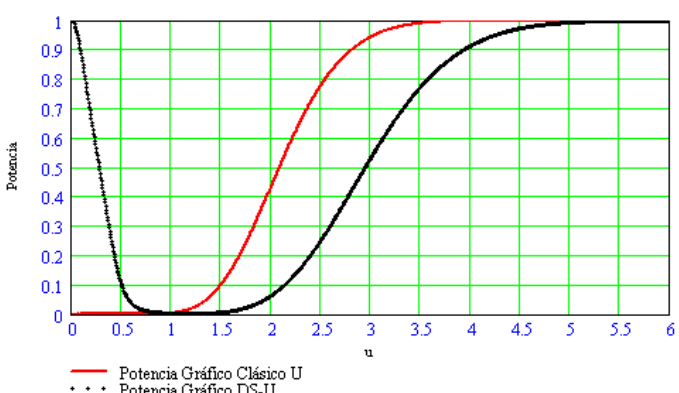

-.. Potencia Gráfico Clásico U

Fig. A.59: Curvas de potencia para $u_{1}=1,0$

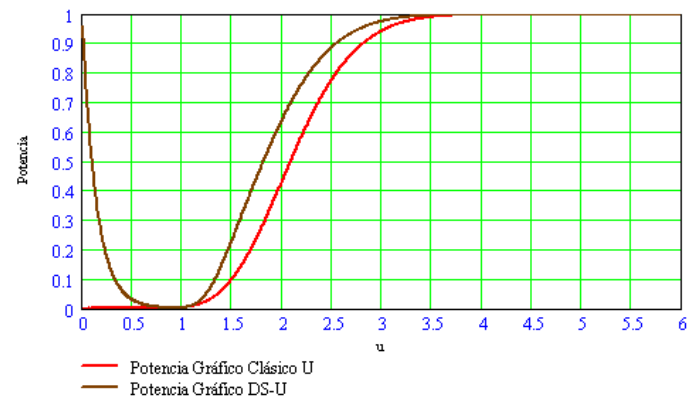

Fig. A.60: Curvas de potencia para $u_{1}=1,1$

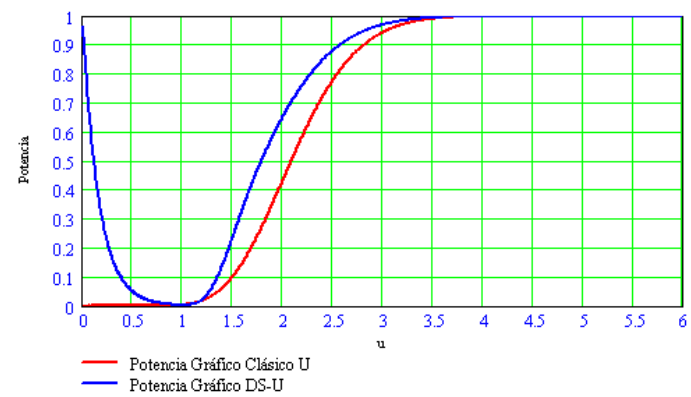

Fig. A.61: Curvas de potencia para $u_{1}=1,2$ 
APÉNDICE A. COMPARACIÓN DE LA POTENCIA DEL GRÁFICO U FRENTE A LA POTENCIA DEL GRÁFICO DS-U EN UN VALOR U

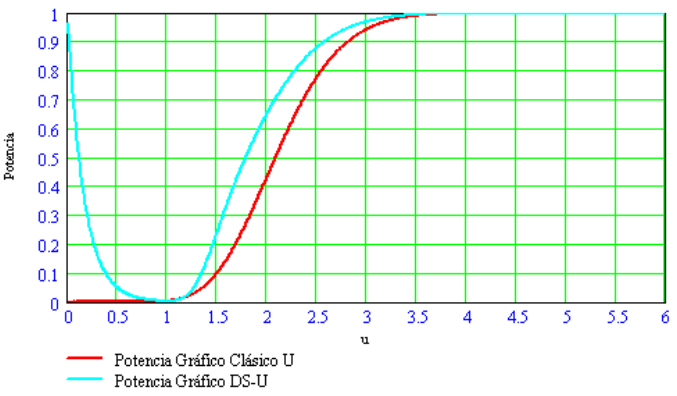

Fig. A.62: Curvas de potencia para $u_{1}=1,3$

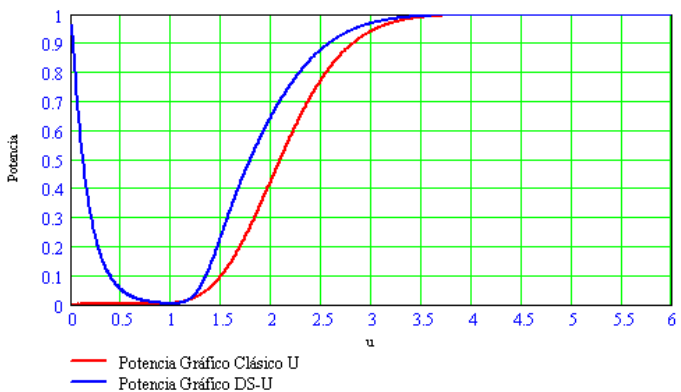

Fig. A.63: Curvas de potencia para $u_{1}=1,4$

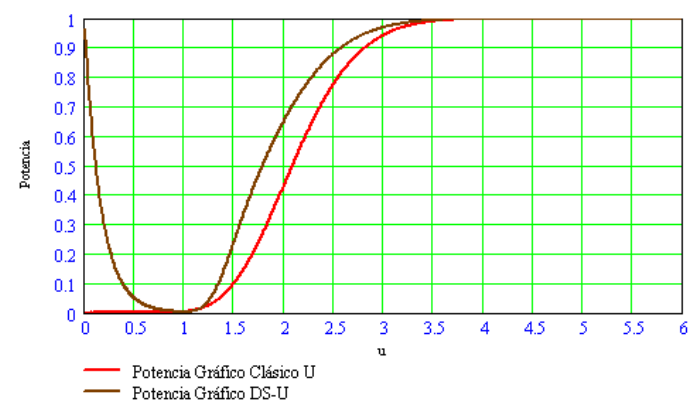

Fig. A.64: Curvas de potencia para $u_{1}=1,5$ 
APÉNDICE A. COMPARACIÓN DE LA POTENCIA DEL GRÁFICO U FRENTE A LA POTENCIA DEL GRÁFICO DS-U EN UN VALOR U

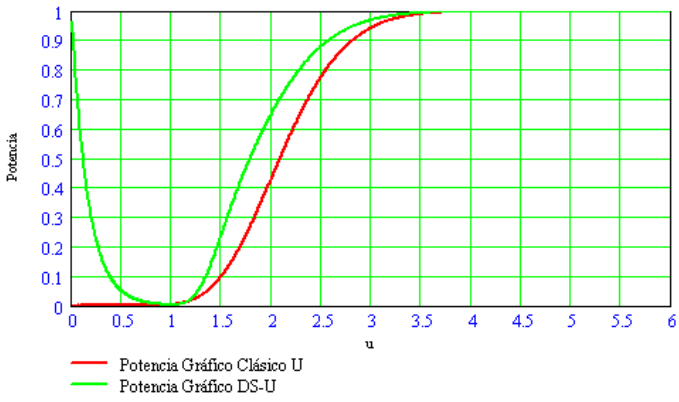

Fig. A.65: Curvas de potencia para $u_{1}=1,6$

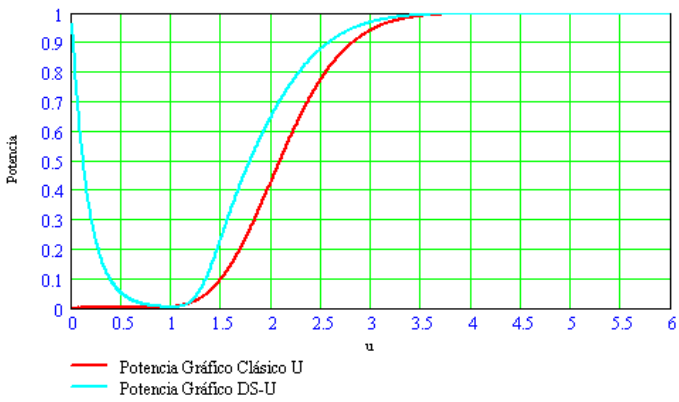

Fig. A.66: Curvas de potencia para $u_{1}=1,7$

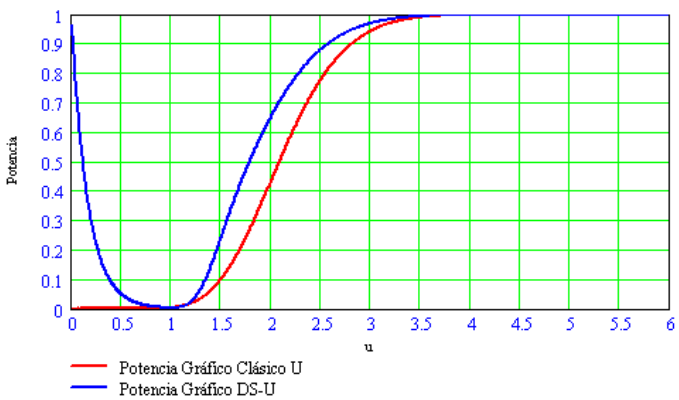

Fig. A.67: Curvas de potencia para $u_{1}=1,8$ 
APÉNDICE A. COMPARACIÓN DE LA POTENCIA DEL GRÁFICO U FRENTE A LA POTENCIA DEL GRÁFICO DS-U EN UN VALOR U

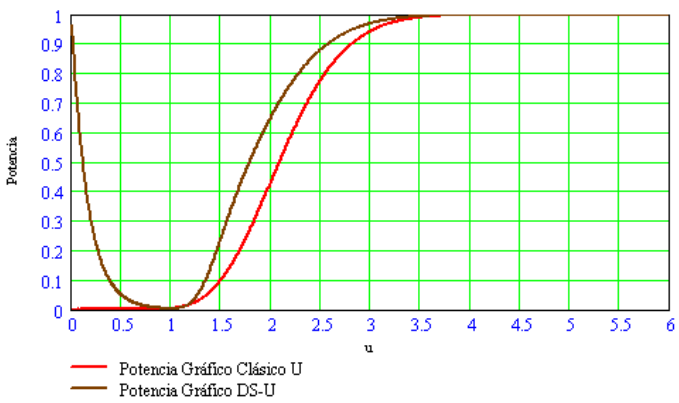

Fig. A.68: Curvas de potencia para $u_{1}=1,9$

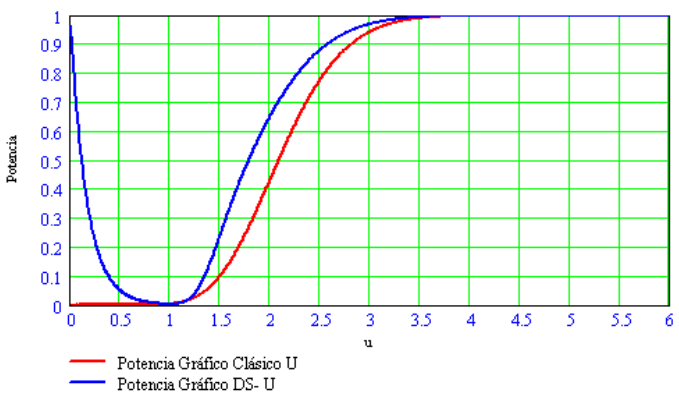

Fig. A.69: Curvas de potencia para $u_{1}=2,0$ 
APÉNDICE A. COMPARACIÓN DE LA POTENCIA DEL GRÁFICO U FRENTE A LA POTENCIA DEL GRÁFICO DS-U EN UN VALOR U

\begin{tabular}{|c||c|c|c|c|c|c||c|c|}
\hline$u_{1}$ & $L C I$ & $L A I$ & $L C I_{1}$ & $L A S$ & $L C S$ & $L C S_{1}$ & $n_{1}$ & $n_{2}$ \\
\hline 0.1 & 0.2 & 2.2 & 18.1 & 44.4 & 55.2 & 55.3 & 6 & 32 \\
0.2 & 0.3 & 2.2 & 20.7 & 34.9 & 56.5 & 52.0 & 6 & 33 \\
0.3 & 0.4 & 2.4 & 20.8 & 32.8 & 36.1 & 51.3 & 6 & 33 \\
0.4 & 0.5 & 2.9 & 20.5 & 30.2 & 55.2 & 53.2 & 6 & 33 \\
0.5 & 0.3 & 2.8 & 14.7 & 14.4 & 25.5 & 46.2 & 6 & 25 \\
0.6 & 0.6 & 2.4 & 20.9 & 15.4 & 51.7 & 53.0 & 6 & 33 \\
0.7 & 0.3 & 2.2 & 19.8 & 23.0 & 31.0 & 41.3 & 7 & 27 \\
0.8 & 0.2 & 2.5 & 23.0 & 19.0 & 47.1 & 54.1 & 7 & 30 \\
0.9 & 0.4 & 2.9 & 25.0 & 16.8 & 43.5 & 46.5 & 7 & 33 \\
\hline 1.0 & 0.4 & 1.0 & 21.3 & 17.3 & 19.8 & 58.2 & 6 & 47 \\
\hline 1.1 & 0.4 & 0.5 & 12.2 & 12.8 & 52.0 & 44.8 & 7 & 36 \\
1.2 & 0.2 & 0.7 & 3.0 & 10.6 & 20.3 & 57.2 & 6 & 43 \\
1.3 & 0.3 & 0.6 & 16.2 & 10.1 & 30.9 & 58.4 & 6 & 44 \\
1.4 & 0.2 & 0.3 & 5.2 & 10.5 & 19.0 & 58.8 & 6 & 44 \\
1.5 & 0.3 & 0.6 & 11.4 & 10.6 & 19.2 & 58.1 & 6 & 44 \\
1.6 & 0.4 & 0.5 & 13.8 & 10.3 & 19.5 & 58.8 & 6 & 44 \\
1.7 & 0.4 & 0.9 & 12.2 & 10.2 & 19.7 & 58.7 & 6 & 44 \\
1.8 & 0.2 & 0.6 & 3.6 & 10.9 & 19.6 & 58.1 & 6 & 44 \\
1.9 & 0.7 & 0.8 & 19.0 & 10.7 & 19.9 & 58.0 & 6 & 44 \\
2.0 & 0.2 & 0.9 & 13.6 & 10.2 & 19.2 & 58.3 & 6 & 44 \\
\hline
\end{tabular}

Tabla A.5: Parámetros obtenidos en la comparación de la potencia para el nuevo gráfico $D S-U: u_{0}=1, \alpha_{t}=0.0027, n=8$ 
APÉNDICE A. COMPARACIÓN DE LA POTENCIA DEL GRÁFICO U FRENTE A LA POTENCIA DEL GRÁFICO DS-U EN UN VALOR U

\begin{tabular}{|c||c|c||c|c|}
\hline$u_{1}$ & Pot $_{u}$ & Pot $_{D S-U}$ & Pot $_{D S-U}-$ Pot $_{U}$ & $\alpha_{\text {real }}$ \\
\hline 0.1 & 0 & 0.976885 & 0.976885 & $2.793736 \cdot 10^{-3}$ \\
0.2 & $1.837863 \cdot 10^{-12}$ & 0.879484 & 0.879484 & $3.149286 \cdot 10^{-3}$ \\
0.3 & $8.547522 \cdot 10^{-10}$ & 0.729824 & 0.729824 & $3.180822 \cdot 10^{-3}$ \\
0.4 & $5.379347 \cdot 10^{-8}$ & 0.555944 & 0.555944 & $3.129651 \cdot 10^{-3}$ \\
0.5 & $1.132832 \cdot 10^{-6}$ & 0.320517 & 0.320516 & $3.237293 \cdot 10^{-3}$ \\
0.6 & $1.194899 \cdot 10^{-5}$ & 0.186203 & 0.186191 & $3.135221 \cdot 10^{-3}$ \\
0.7 & $7.829316 \cdot 10^{-5}$ & 0.079166 & 0.079087 & $3.014416 \cdot 10^{-3}$ \\
0.8 & $3.624098 \cdot 10^{-4}$ & 0.034524 & 0.034161 & $3.237414 \cdot 10^{-3}$ \\
0.9 & $1.287762 \cdot 10^{-3}$ & 0.010168 & $8.879818 \cdot 10^{-3}$ & $3.189711 \cdot 10^{-3}$ \\
\hline 1.0 & $3.718021 \cdot 10^{-3}$ & $3.239992 \cdot 10^{-3}$ & $-4.780294 \cdot 10^{-4}$ & $3.239992 \cdot 10^{-3}$ \\
\hline 1.1 & $9.084083 \cdot 10^{-3}$ & 0.011291 & $2.207246 \cdot 10^{-3}$ & $3.122320 \cdot 10^{-3}$ \\
1.2 & 0.019357 & 0.023824 & $4.467361 \cdot 10^{-3}$ & $3.194258 \cdot 10^{-3}$ \\
1.3 & 0.036808 & 0.070171 & 0.033363 & $3.233008 \cdot 10^{-3}$ \\
1.4 & 0.063597 & 0.146387 & 0.082790 & $3.238048 \cdot 10^{-3}$ \\
1.5 & 0.101291 & 0.241804 & 0.140513 & $3.238048 \cdot 10^{-3}$ \\
1.6 & 0.150465 & 0.339823 & 0.189358 & $3.238050 \cdot 10^{-3}$ \\
1.7 & 0.210503 & 0.430543 & 0.220040 & $3.238049 \cdot 10^{-3}$ \\
1.8 & 0.279636 & 0.512113 & 0.232476 & $3.238048 \cdot 10^{-3}$ \\
1.9 & 0.355203 & 0.585789 & 0.230586 & $3.238375 \cdot 10^{-3}$ \\
2.0 & 0.434038 & 0.652504 & 0.218467 & $3.238050 \cdot 10^{-3}$ \\
\hline
\end{tabular}

Tabla A.6: Resultados para $u_{0}=1, \alpha_{\text {teórico }}=0.0027$ y $n=8$ 
APÉNDICE A. COMPARACIÓN DE LA POTENCIA DEL GRÁFICO U FRENTE A LA POTENCIA DEL GRÁFICO DS-U EN UN VALOR U

A.4. Comparación de los gráficos de control $u$ y $\boldsymbol{D S}-\boldsymbol{U} \operatorname{con} n=40, u_{0}=1$ y $\alpha_{t}=0.01$ 
APÉNDICE A. COMPARACIÓN DE LA POTENCIA DEL GRÁFICO U FRENTE A LA POTENCIA DEL GRÁFICO DS-U EN UN VALOR U

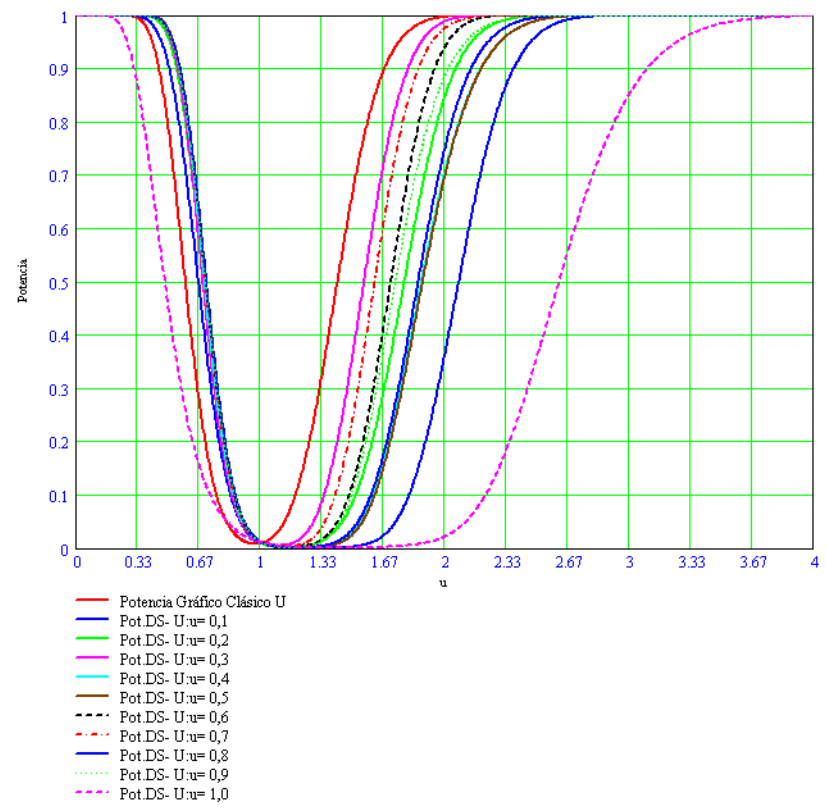

(a) $u_{1} \in[0.1,1.0]$

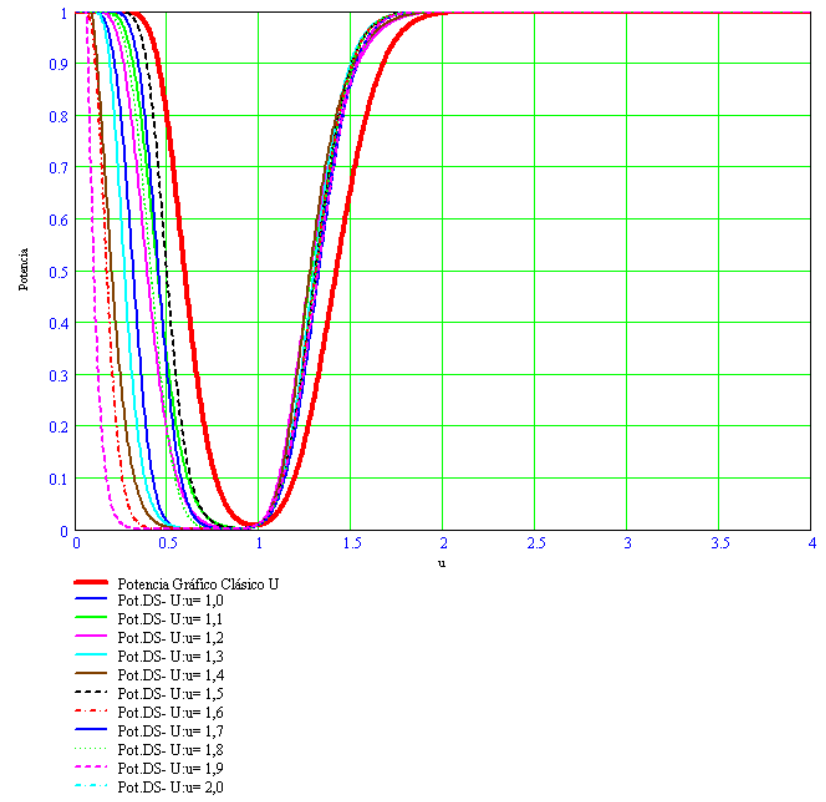

(b) $u_{1} \in[1.1,2.0]$

Fig. A.70: Curvas de potencia en distintos valores de $u_{1}$ 
APÉNDICE A. COMPARACIÓN DE LA POTENCIA DEL GRÁFICO U FRENTE A LA POTENCIA DEL GRÁFICO DS-U EN UN VALOR U

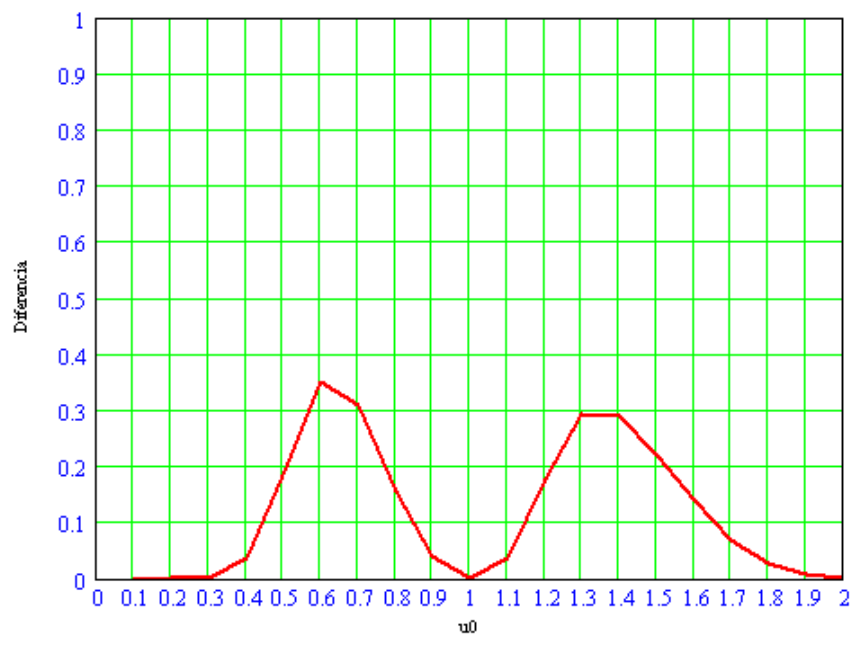

Fig. A.71: $\operatorname{Pot}_{D S-U}-\operatorname{Pot}_{u}$ para $n=40, u_{0}=1$ y $\alpha_{t}=0.01$

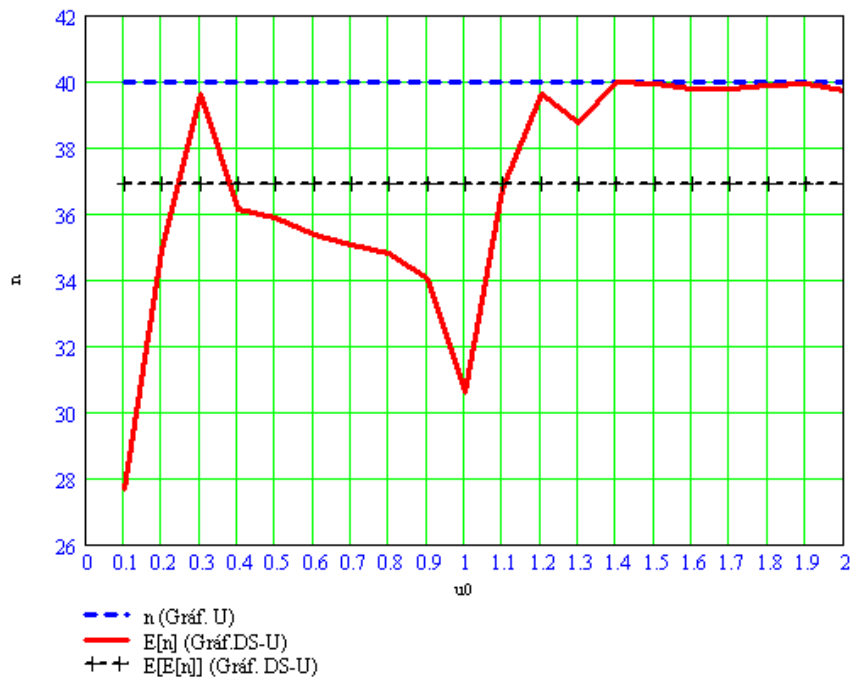

Fig. A.72: Tamaño medio de muestra en el gráfico $D S-U$ para $n=40, u_{0}=1$ y $\alpha_{t}=0.01$ 
APÉNDICE A. COMPARACIÓN DE LA POTENCIA DEL GRÁFICO U FRENTE A LA POTENCIA DEL GRÁFICO DS-U EN UN VALOR U

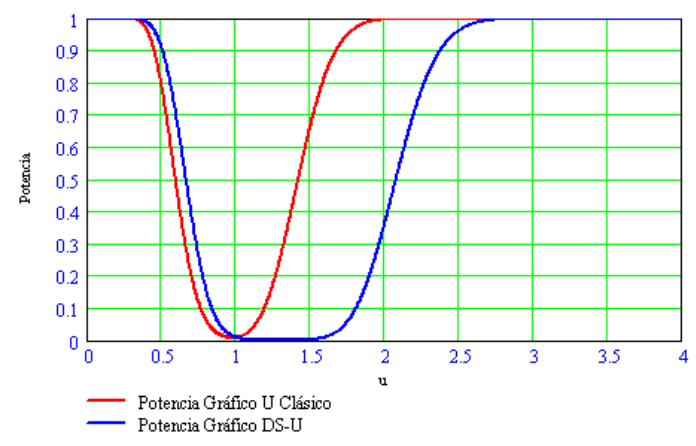

Fig. A.73: Curvas de potencia para $u_{1}=0,1$

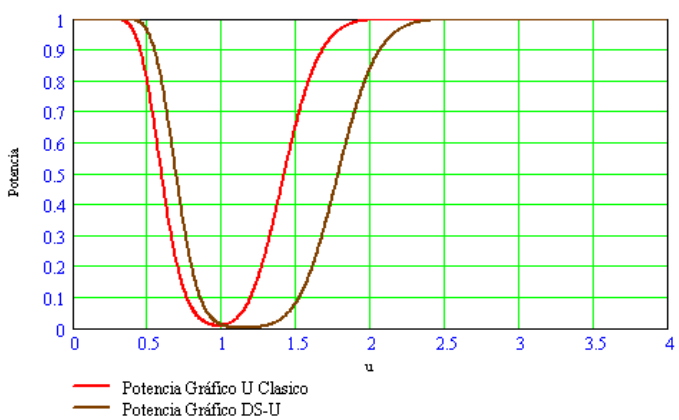

Fig. A.74: Curvas de potencia para $u_{1}=0,2$

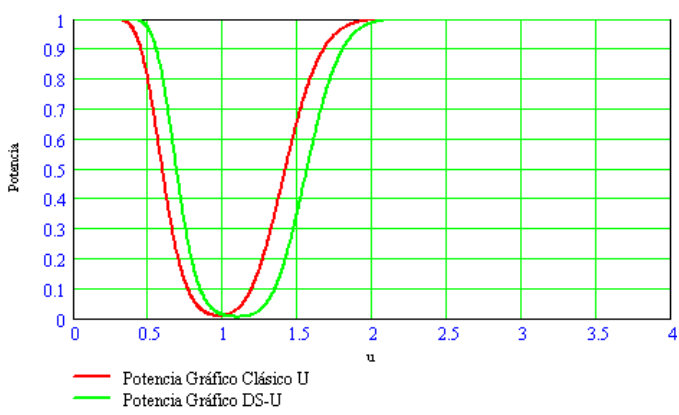

Fig. A.75: Curvas de potencia para $u_{1}=0,3$ 
APÉNDICE A. COMPARACIÓN DE LA POTENCIA DEL GRÁFICO U FRENTE A LA POTENCIA DEL GRÁFICO DS-U EN UN VALOR U

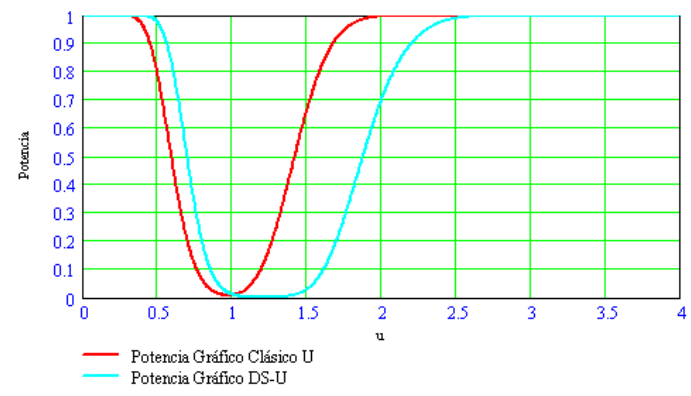

Fig. A.76: Curvas de potencia para $u_{1}=0,4$

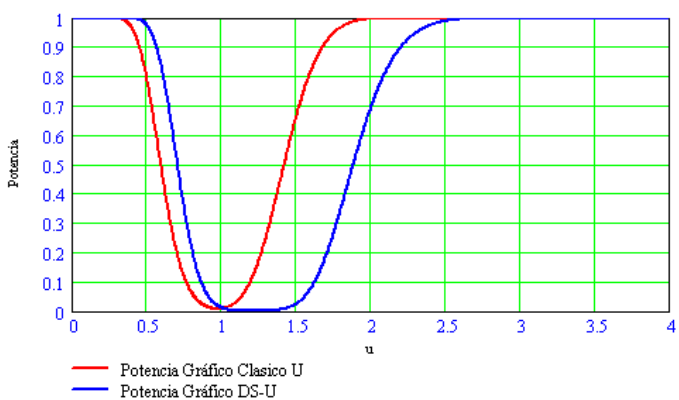

Fig. A.77: Curvas de potencia para $u_{1}=0,5$

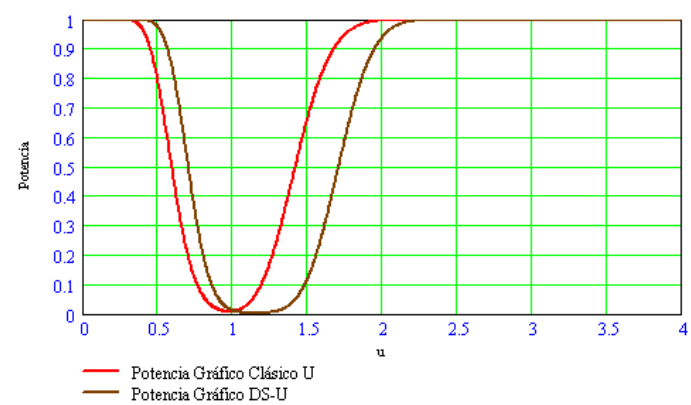

Fig. A.78: Curvas de potencia para $u_{1}=0,6$ 
APÉNDICE A. COMPARACIÓN DE LA POTENCIA DEL GRÁFICO U FRENTE A LA POTENCIA DEL GRÁFICO DS-U EN UN VALOR U

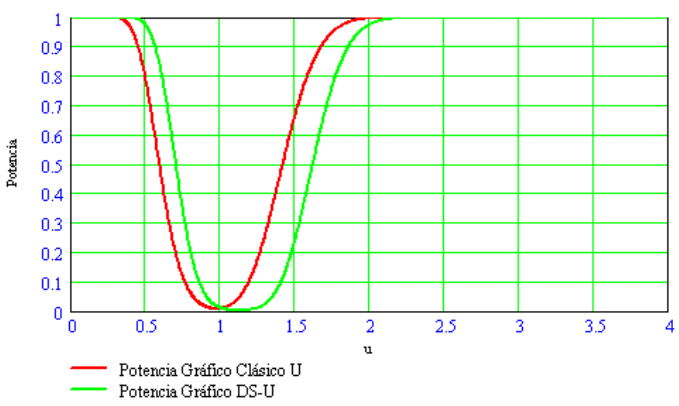

Fig. A.79: Curvas de potencia para $u_{1}=0,7$

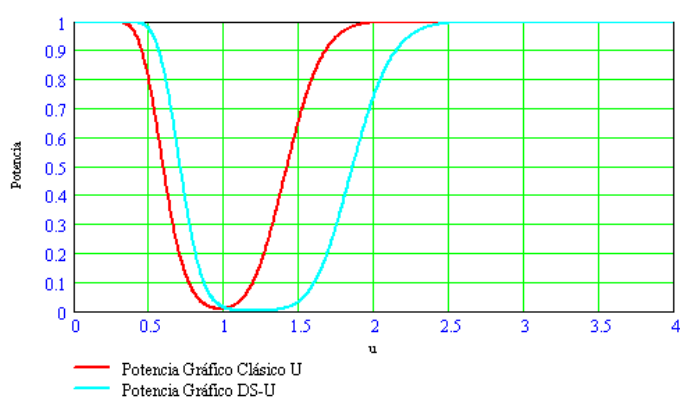

Fig. A.80: Curvas de potencia para $u_{1}=0,8$

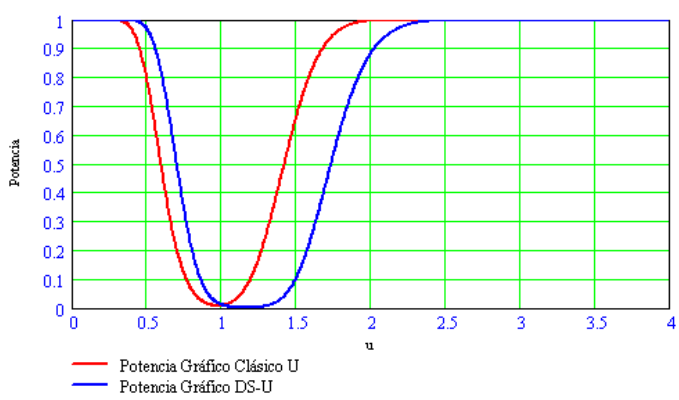

Fig. A.81: Curvas de potencia para $u_{1}=0,9$ 
APÉNDICE A. COMPARACIÓN DE LA POTENCIA DEL GRÁFICO U FRENTE A LA POTENCIA DEL GRÁFICO DS-U EN UN VALOR U

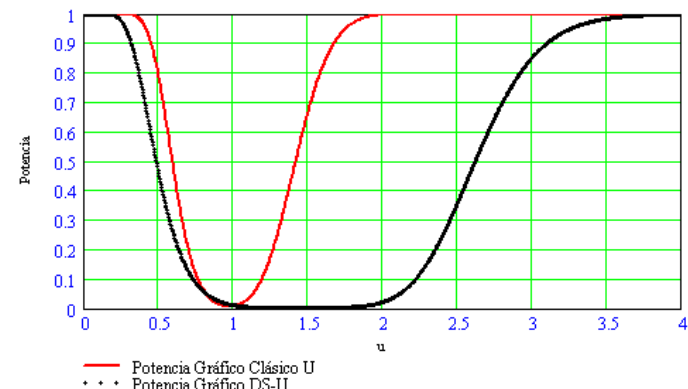

- Potencia Gráfico Clásico

Fig. A.82: Curvas de potencia para $u_{1}=1,0$

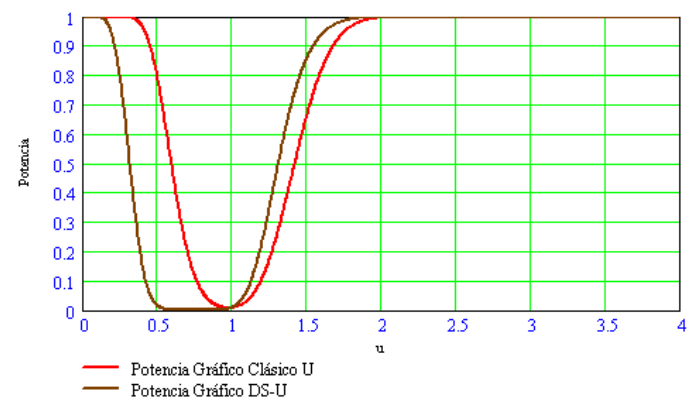

Fig. A.83: Curvas de potencia para $u_{1}=1,1$

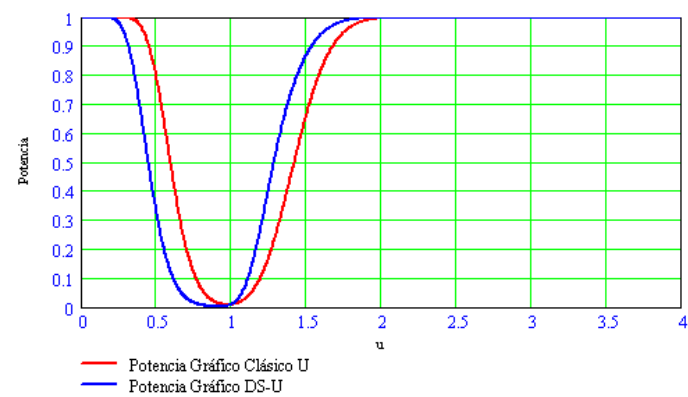

Fig. A.84: Curvas de potencia para $u_{1}=1,2$ 
APÉNDICE A. COMPARACIÓN DE LA POTENCIA DEL GRÁFICO U FRENTE A LA POTENCIA DEL GRÁFICO DS-U EN UN VALOR U

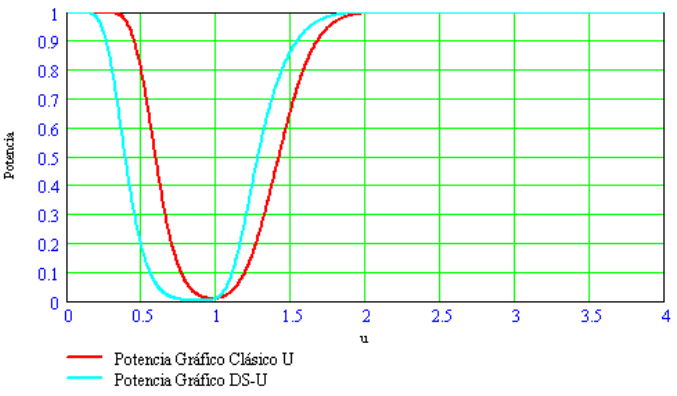

Fig. A.85: Curvas de potencia para $u_{1}=1,3$

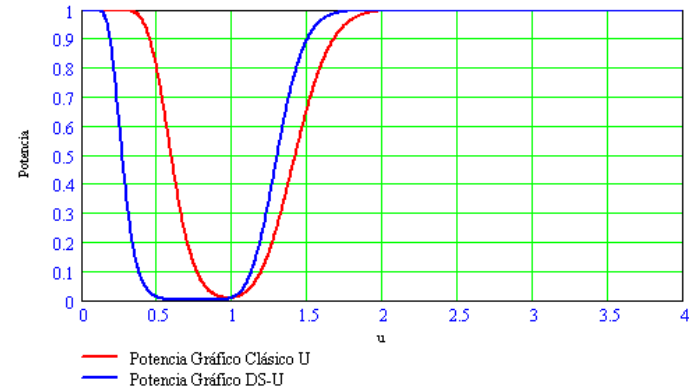

Fig. A.86: Curvas de potencia para $u_{1}=1,4$

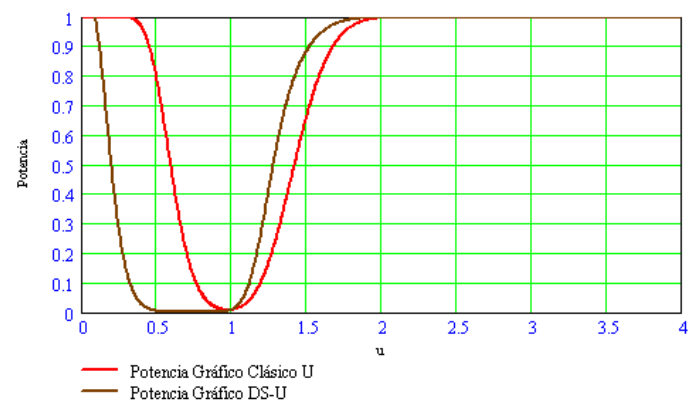

Fig. A.87: Curvas de potencia para $u_{1}=1,5$ 
APÉNDICE A. COMPARACIÓN DE LA POTENCIA DEL GRÁFICO U FRENTE A LA POTENCIA DEL GRÁFICO DS-U EN UN VALOR U

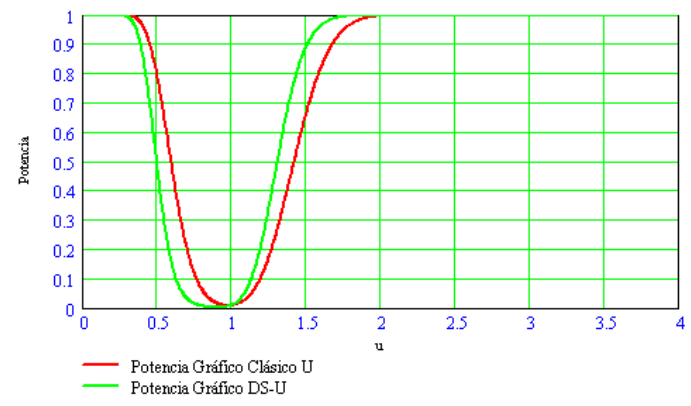

Fig. A.88: Curvas de potencia para $u_{1}=1,6$

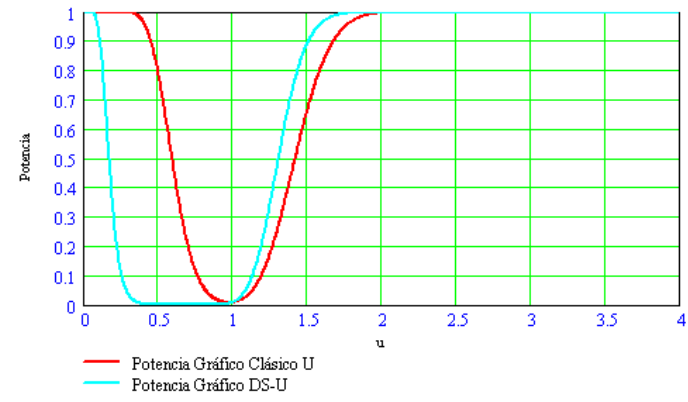

Fig. A.89: Curvas de potencia para $u_{1}=1,7$

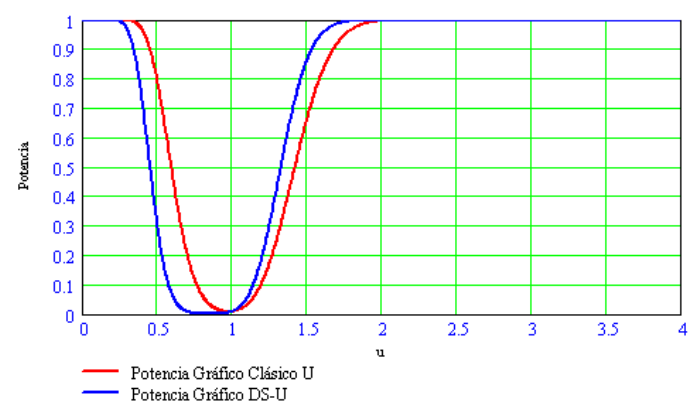

Fig. A.90: Curvas de potencia para $u_{1}=1,8$ 
APÉNDICE A. COMPARACIÓN DE LA POTENCIA DEL GRÁFICO U FRENTE A LA POTENCIA DEL GRÁFICO DS-U EN UN VALOR U

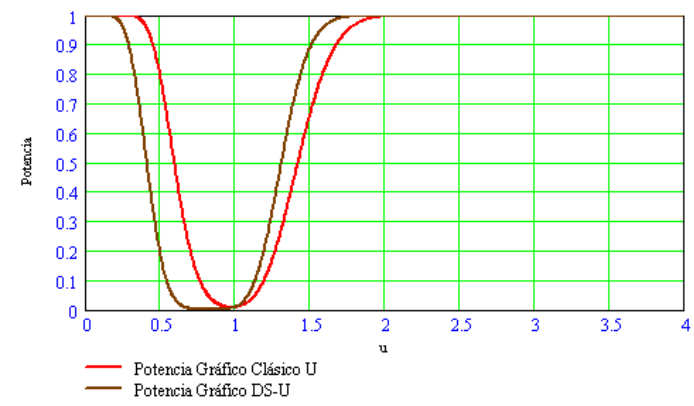

Fig. A.91: Curvas de potencia para $u_{1}=1,9$

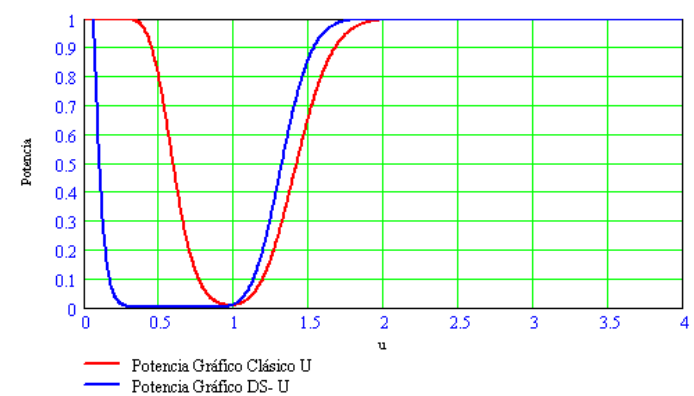

Fig. A.92: Curvas de potencia para $u_{1}=2,0$ 
APÉNDICE A. COMPARACIÓN DE LA POTENCIA DEL GRÁFICO U FRENTE A LA POTENCIA DEL GRÁFICO DS-U EN UN VALOR U

\begin{tabular}{|c||c|c|c|c|c|c||c|c|}
\hline$u_{1}$ & $L C I$ & $L A I$ & $L C I_{1}$ & $L A S$ & $L C S$ & $L C S_{1}$ & $n_{1}$ & $n_{2}$ \\
\hline 0.1 & 6.5 & 18.5 & 20.4 & 49.4 & 58.8 & 53.2 & 26 & 26 \\
0.2 & 12.8 & 24.2 & 22.9 & 50.6 & 54.7 & 58.9 & 30 & 31 \\
0.3 & 15.2 & 24.1 & 24.8 & 36.3 & 55.0 & 56.9 & 28 & 37 \\
0.4 & 16.8 & 24.5 & 24.2 & 53.5 & 57.9 & 59.2 & 29 & 36 \\
0.5 & 14.6 & 24.7 & 24.1 & 53.1 & 58.8 & 56.0 & 29 & 34 \\
0.6 & 16.3 & 24.0 & 25.0 & 43.7 & 56.9 & 59.5 & 31 & 33 \\
0.7 & 16.9 & 24.1 & 24.6 & 45.4 & 57.6 & 51.3 & 31 & 33 \\
0.8 & 14.9 & 24.4 & 24.5 & 55.4 & 59.6 & 58.0 & 31 & 32 \\
0.9 & 12.7 & 23.3 & 24.7 & 48.1 & 55.6 & 51.8 & 29 & 33 \\
\hline 1.0 & 5.1 & 17.4 & 6.3 & 32.0 & 45.5 & 47.5 & 13 & 20 \\
\hline 1.1 & 5.6 & 10.0 & 21.5 & 41.7 & 55.5 & 58.9 & 33 & 51 \\
1.2 & 14.1 & 14.2 & 62.7 & 41.7 & 52.5 & 101.0 & 33 & 91 \\
1.3 & 11.4 & 11.7 & 15.9 & 37.1 & 47.0 & 112.2 & 30 & 100 \\
1.4 & 7.4 & 17.7 & 9.1 & 37.2 & 49.0 & 59.2 & 32 & 48 \\
1.5 & 5.0 & 12.5 & 7.2 & 35.9 & 43.1 & 116.7 & 29 & 99 \\
1.6 & 12.7 & 17.1 & 22.2 & 35.0 & 46.4 & 59.2 & 30 & 47 \\
1.7 & 3.8 & 17.7 & 6.3 & 34.6 & 46.3 & 59.0 & 30 & 47 \\
1.8 & 1.2 & 15.2 & 20.9 & 28.1 & 41.9 & 57.1 & 26 & 44 \\
1.9 & 1.4 & 13.8 & 24.5 & 38.8 & 49.5 & 53.5 & 33 & 42 \\
2.0 & 1.0 & 9.7 & 2.6 & 30.1 & 44.9 & 49.1 & 28 & 38 \\
\hline
\end{tabular}

Tabla A.7: Parámetros obtenidos en la comparación de la potencia para el nuevo gráfico $D S-U: u_{0}=1, \alpha_{t}=0.01, n=40$ 
APÉNDICE A. COMPARACIÓN DE LA POTENCIA DEL GRÁFICO U FRENTE A LA POTENCIA DEL GRÁFICO DS-U EN UN VALOR U

\begin{tabular}{|c||c|c||c|c|}
\hline$u_{1}$ & Pot $_{U}$ & Pot $_{D S-U}$ & Pot $_{D S-U}-$ Pot $_{U}$ & $\alpha_{\text {real }}$ \\
\hline 0.1 & 1 & 1 & $-4.387235 \cdot 10^{-11}$ & $8.967257 \cdot 10^{-3}$ \\
0.2 & 0.999996 & 1 & $3.718023 \cdot 10^{-6}$ & $9.277931 \cdot 10^{-3}$ \\
0.3 & 0.998527 & 0.999994 & $1.467320 \cdot 10^{-3}$ & 0.010657 \\
0.4 & 0.963314 & 0.999008 & 0.035694 & 0.010792 \\
0.5 & 0.787493 & 0.973106 & 0.185613 & 0.010949 \\
0.6 & 0.472850 & 0.8245331 & 0.351684 & 0.010861 \\
0.7 & 0.199810 & 0.508132 & 0.308323 & 0.010432 \\
0.8 & 0.061012 & 0.216470 & 0.155458 & 0.010950 \\
0.9 & 0.014796 & 0.054977 & 0.040181 & 0.010339 \\
\hline 1.0 & $9.131637 \cdot 10^{-3}$ & 0.010958 & $1.826762 \cdot 10^{-3}$ & 0.010958 \\
\hline 1.1 & 0.034119 & 0.071322 & 0.037203 & 0.010958 \\
1.2 & 0.111937 & 0.291179 & 0.179242 & 0.010940 \\
1.3 & 0.261682 & 0.555428 & 0.293746 & 0.010934 \\
1.4 & 0.464541 & 0.754474 & 0.289933 & 0.010600 \\
1.5 & 0.668064 & 0.888786 & 0.220722 & 0.010295 \\
1.6 & 0.825224 & 0.966030 & 0.140806 & 0.010517 \\
1.7 & 0.921623 & 0.990153 & 0.068529 & 0.010347 \\
1.8 & 0.969841 & 0.996677 & 0.026837 & 0.010134 \\
1.9 & 0.989946 & 0.999424 & $9.477359 \cdot 10^{-3}$ & 0.010729 \\
2.0 & 0.997067 & 0.999860 & $2.792507 \cdot 10^{-3}$ & 0.012754 \\
\hline
\end{tabular}

Tabla A.8: Resultados para $u_{0}=1, \alpha_{\text {teórico }}=0.01$ y $n=40$ 
APÉNDICE A. COMPARACIÓN DE LA POTENCIA DEL GRÁFICO U FRENTE A LA POTENCIA DEL GRÁFICO DS-U EN UN VALOR U

A.5. Comparación de los gráficos de control $u$ y $\boldsymbol{D} \boldsymbol{S}-\boldsymbol{U} \operatorname{con} n=40, u_{0}=1$ y $\alpha_{t}=0.05$ 
APÉNDICE A. COMPARACIÓN DE LA POTENCIA DEL GRÁFICO U FRENTE A LA POTENCIA DEL GRÁFICO DS-U EN UN VALOR U

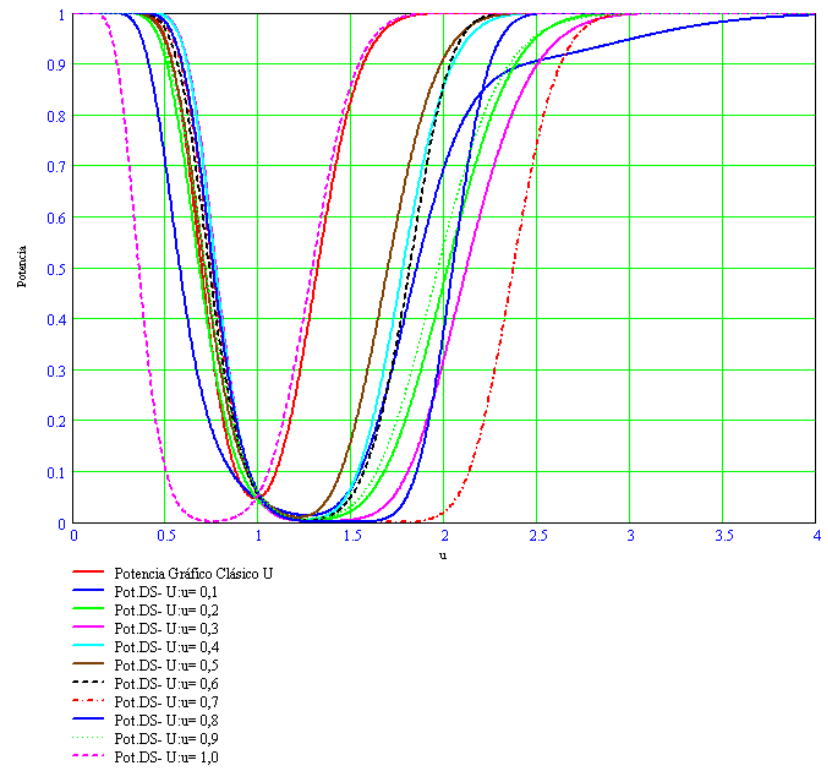

(a) $u_{1} \in[0.1,1.0]$

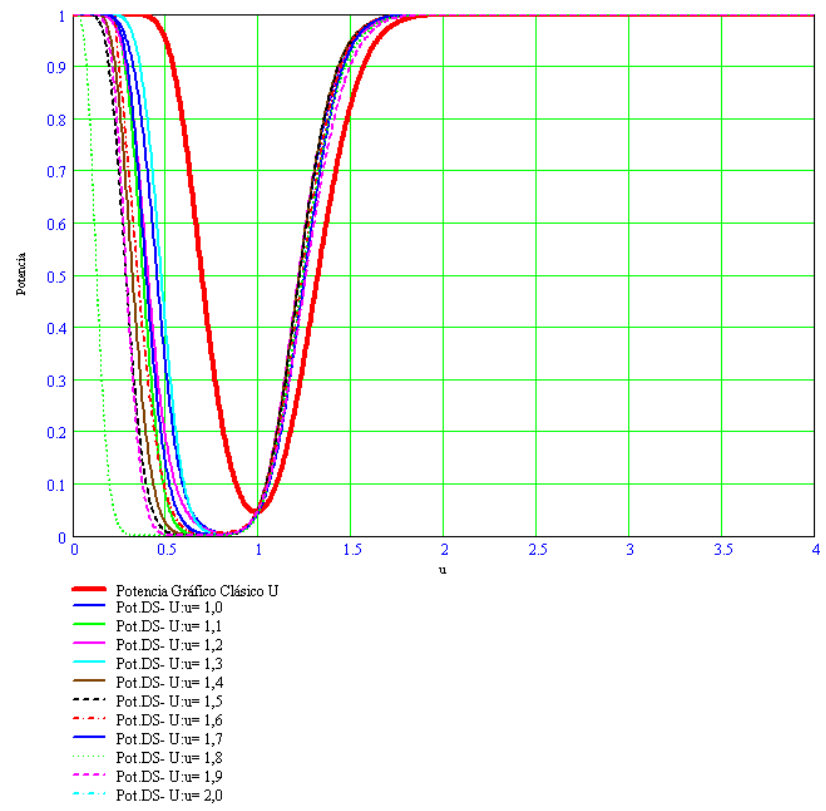

(b) $u_{1} \in[1.1,2.0]$

Fig. A.93: Curvas de potencia en distintos valores de $u_{1}$ 
APÉNDICE A. COMPARACIÓN DE LA POTENCIA DEL GRÁFICO U FRENTE A LA POTENCIA DEL GRÁFICO DS-U EN UN VALOR U

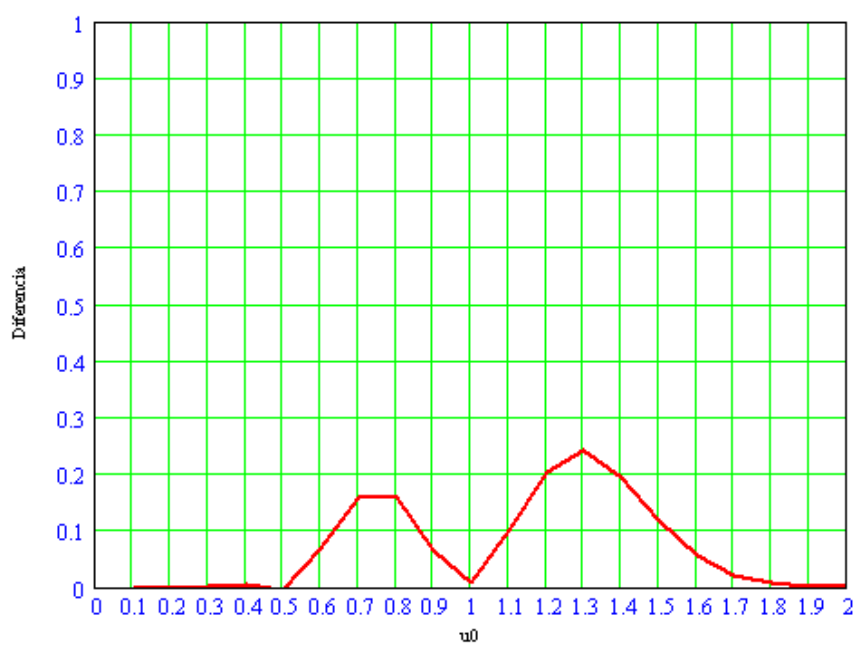

Fig. A.94: $\operatorname{Pot}_{D S-U}-\operatorname{Pot}_{u}$ para $n=40, u_{0}=1$ y $\alpha_{t}=0.05$

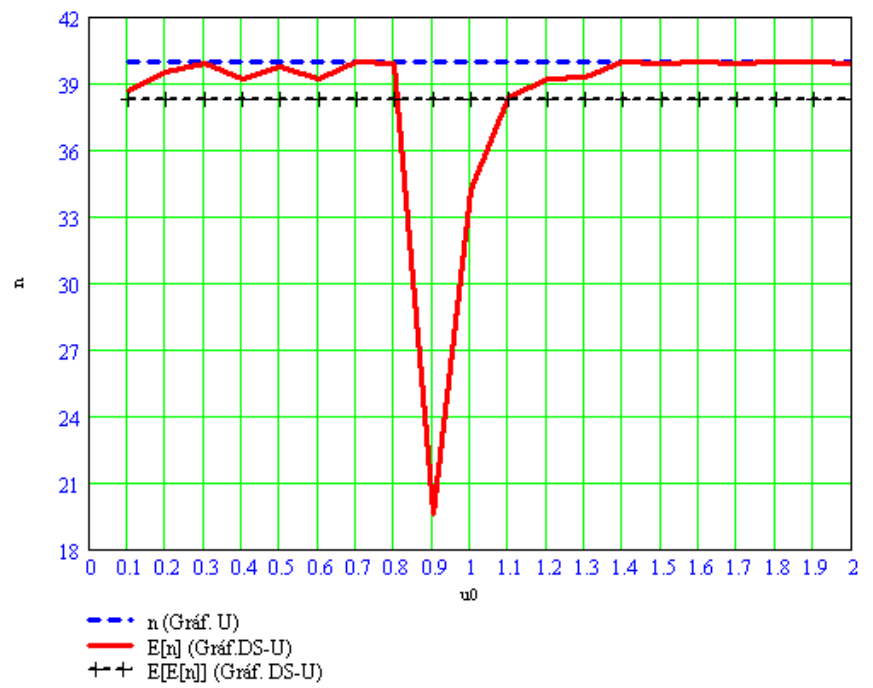

Fig. A.95: Tamaño medio de muestra en el gráfico $D S-U$ para $n=40, u_{0}=1$ y $\alpha_{t}=0.05$ 
APÉNDICE A. COMPARACIÓN DE LA POTENCIA DEL GRÁFICO U FRENTE A LA POTENCIA DEL GRÁFICO DS-U EN UN VALOR U

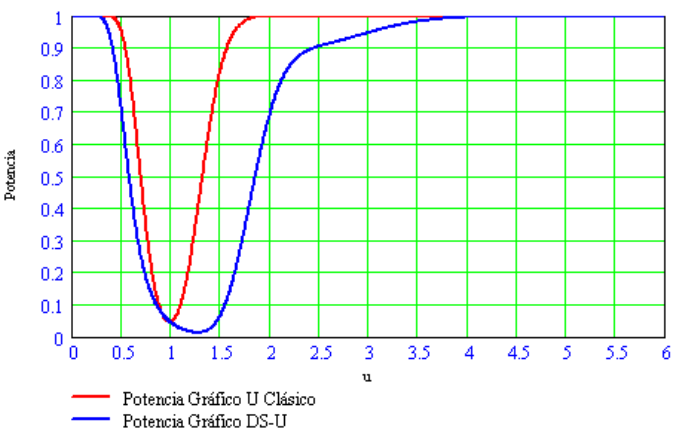

Fig. A.96: Curvas de potencia para $u_{1}=0,1$

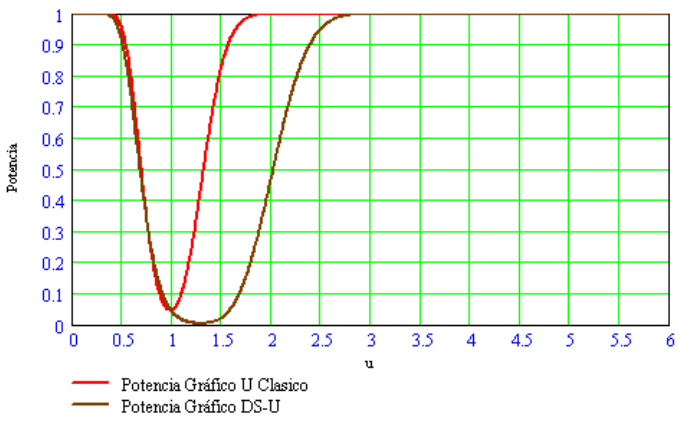

Fig. A.97: Curvas de potencia para $u_{1}=0,2$

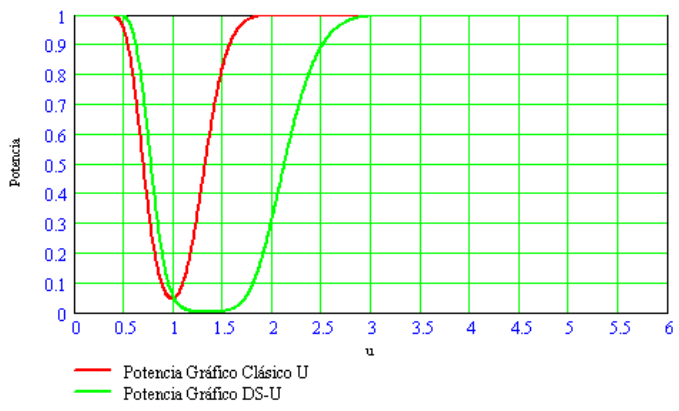

Fig. A.98: Curvas de potencia para $u_{1}=0,3$ 
APÉNDICE A. COMPARACIÓN DE LA POTENCIA DEL GRÁFICO U FRENTE A LA POTENCIA DEL GRÁFICO DS-U EN UN VALOR U

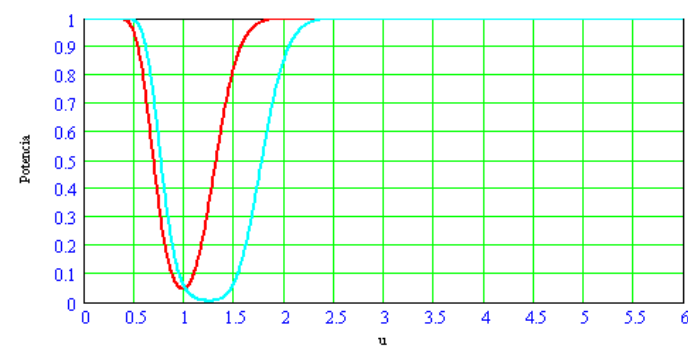

- Potencia Gráfico Clásico U

Potencia Gráfico DS-U

Fig. A.99: Curvas de potencia para $u_{1}=0,4$

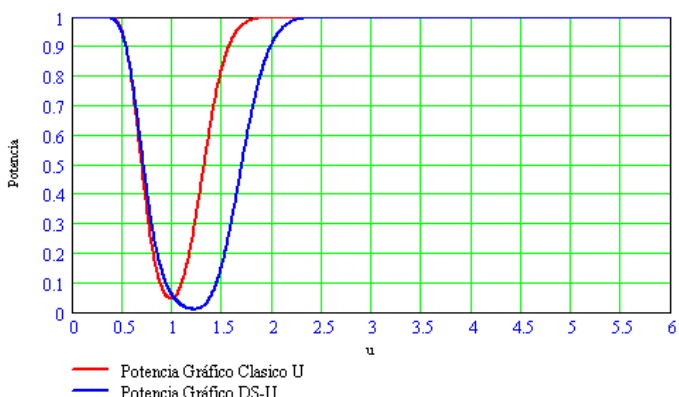

Fig. A.100: Curvas de potencia para $u_{1}=0,5$

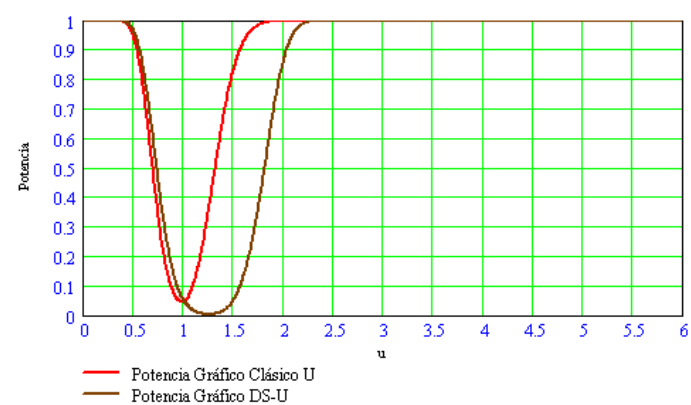

Fig. A.101: Curvas de potencia para $u_{1}=0,6$ 
APÉNDICE A. COMPARACIÓN DE LA POTENCIA DEL GRÁFICO U FRENTE A LA POTENCIA DEL GRÁFICO DS-U EN UN VALOR U

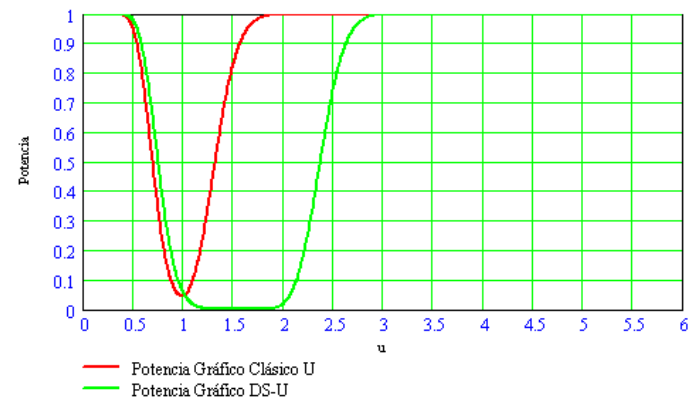

Fig. A.102: Curvas de potencia para $u_{1}=0,7$

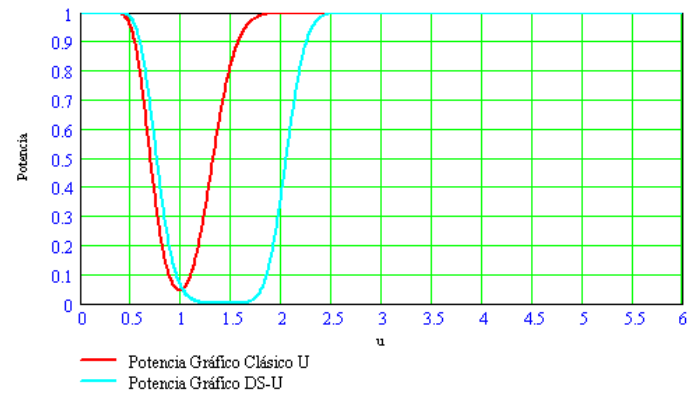

Fig. A.103: Curvas de potencia para $u_{1}=0,8$

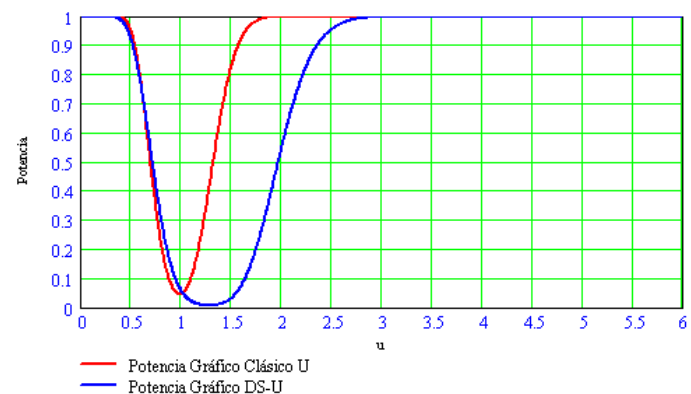

Fig. A.104: Curvas de potencia para $u_{1}=0,9$ 
APÉNDICE A. COMPARACIÓN DE LA POTENCIA DEL GRÁFICO U FRENTE A LA POTENCIA DEL GRÁFICO DS-U EN UN VALOR U

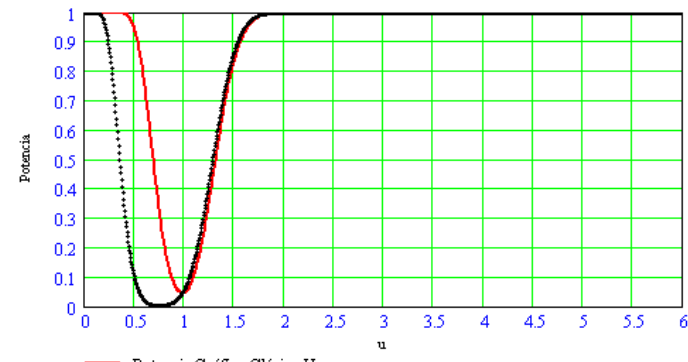

-.. Potencia Gráfico Clásico U

Fig. A.105: Curvas de potencia para $u_{1}=1,0$

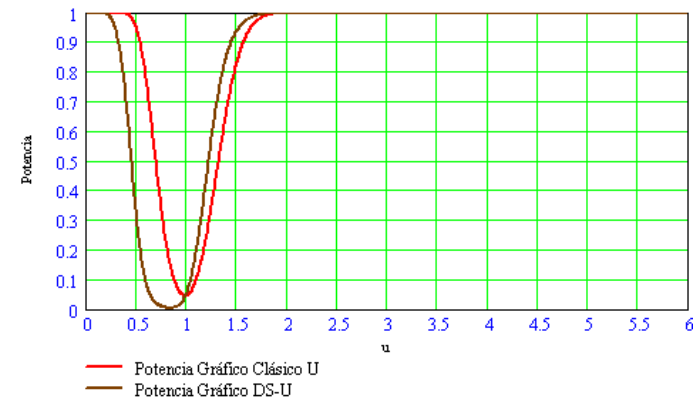

Fig. A.106: Curvas de potencia para $u_{1}=1,1$

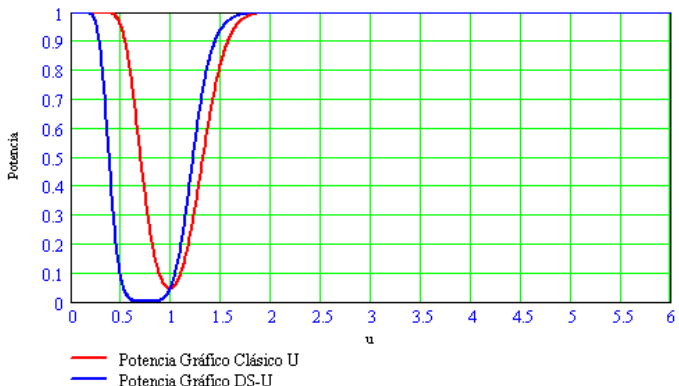

Fig. A.107: Curvas de potencia para $u_{1}=1,2$ 
APÉNDICE A. COMPARACIÓN DE LA POTENCIA DEL GRÁFICO U FRENTE A LA POTENCIA DEL GRÁFICO DS-U EN UN VALOR U

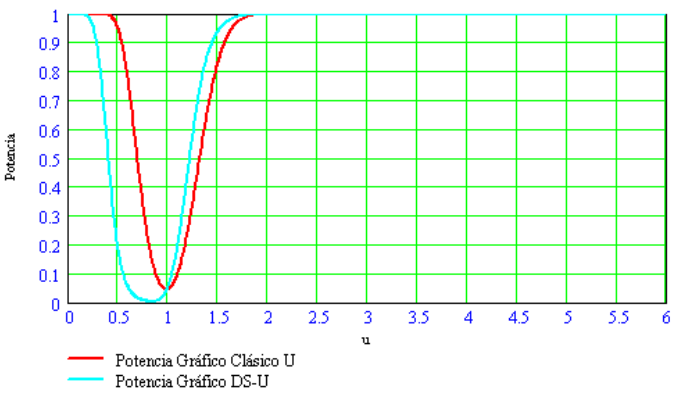

Fig. A.108: Curvas de potencia para $u_{1}=1,3$

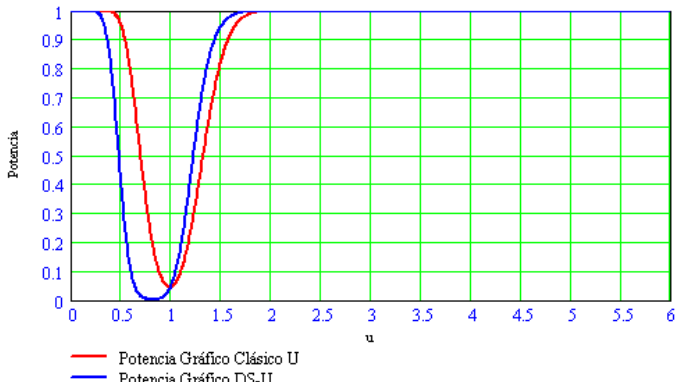

— Potencia Gráfico Clásico U

Fig. A.109: Curvas de potencia para $u_{1}=1,4$

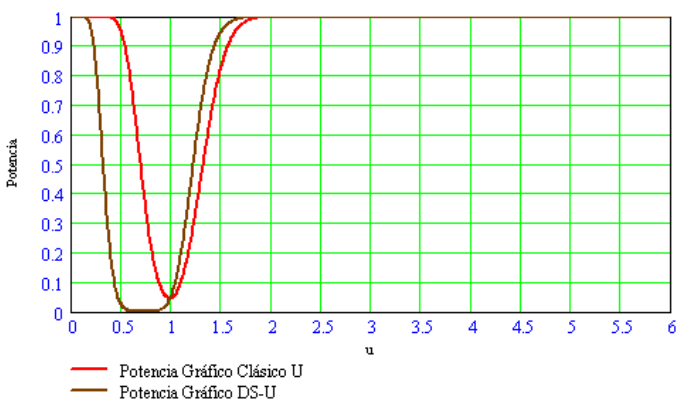

Fig. A.110: Curvas de potencia para $u_{1}=1,5$ 
APÉNDICE A. COMPARACIÓN DE LA POTENCIA DEL GRÁFICO U FRENTE A LA POTENCIA DEL GRÁFICO DS-U EN UN VALOR U

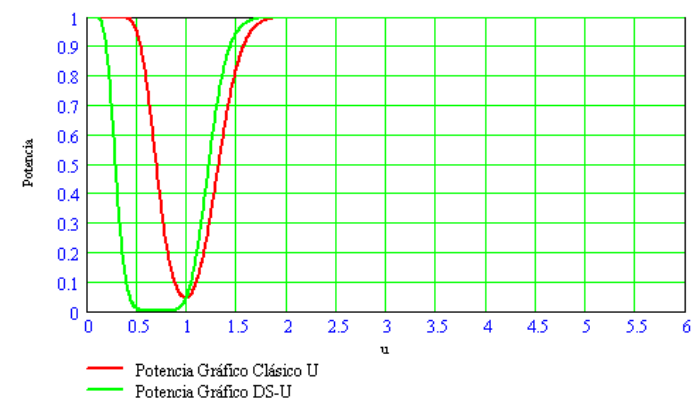

Fig. A.111: Curvas de potencia para $u_{1}=1,6$

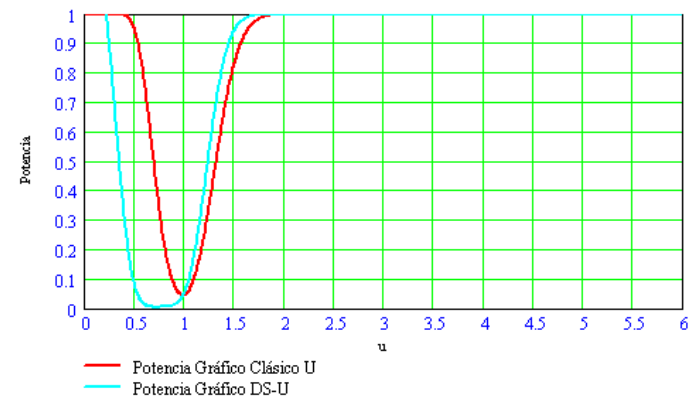

Fig. A.112: Curvas de potencia para $u_{1}=1,7$

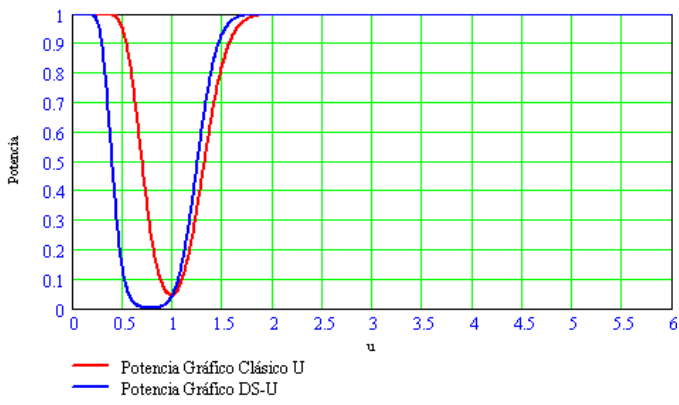

Fig. A.113: Curvas de potencia para $u_{1}=1,8$ 
APÉNDICE A. COMPARACIÓN DE LA POTENCIA DEL GRÁFICO U FRENTE A LA POTENCIA DEL GRÁFICO DS-U EN UN VALOR U

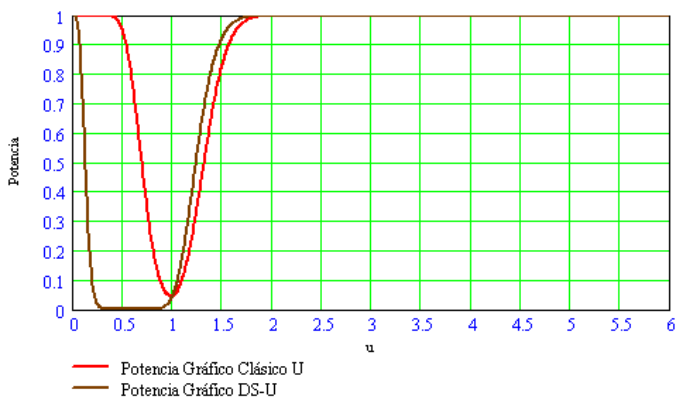

Fig. A.114: Curvas de potencia para $u_{1}=1,9$

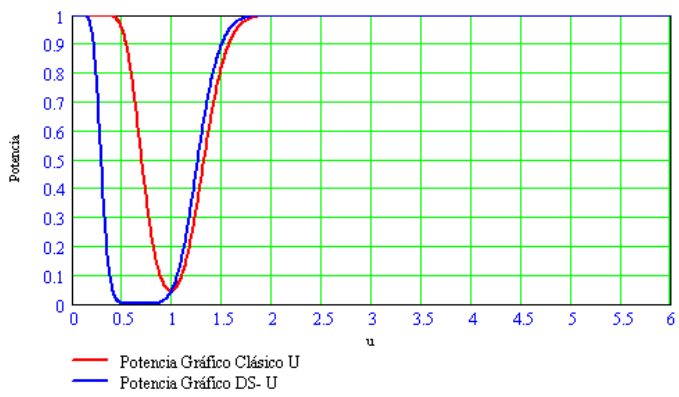

Fig. A.115: Curvas de potencia para $u_{1}=2,0$ 
APÉNDICE A. COMPARACIÓN DE LA POTENCIA DEL GRÁFICO U FRENTE A LA POTENCIA DEL GRÁFICO DS-U EN UN VALOR U

\begin{tabular}{|c||c|c|c|c|c|c||c|c|}
\hline$u_{1}$ & $L C I$ & $L A I$ & $L C I_{1}$ & $L A S$ & $L C S$ & $L C S_{1}$ & $n_{1}$ & $n_{2}$ \\
\hline 0.1 & 3.4 & 18.2 & 15.3 & 20.0 & 45.9 & 58.3 & 8 & 32 \\
0.2 & 8.5 & 15.0 & 15.0 & 15.4 & 43.1 & 48.5 & 16 & 24 \\
0.3 & 15.3 & 24.4 & 25.0 & 52.4 & 55.1 & 59.3 & 25 & 33 \\
0.4 & 15.1 & 23.1 & 25.0 & 36.4 & 54.6 & 58.6 & 24 & 34 \\
0.5 & 7.9 & 13.7 & 14.5 & 23.1 & 46.5 & 48.5 & 15 & 20 \\
0.6 & 8.1 & 14.8 & 14.2 & 25.6 & 35.4 & 34.6 & 17 & 19 \\
0.7 & 6.7 & 13.9 & 13.6 & 31.1 & 44.3 & 32.6 & 17 & 16 \\
0.8 & 9.5 & 14.8 & 13.0 & 28.9 & 36.5 & 29.1 & 18 & 17 \\
0.9 & 5.7 & 12.6 & 13.4 & 26.1 & 34.9 & 31.6 & 15 & 17 \\
\hline 1.0 & 9.3 & 11.4 & 19.1 & 40.7 & 42.6 & 42.3 & 33 & 27 \\
\hline 1.1 & 8.4 & 11.0 & 24.1 & 26.6 & 37.5 & 55.8 & 24 & 49 \\
1.2 & 2.2 & 11.7 & 19.9 & 27.9 & 38.5 & 54.1 & 25 & 48 \\
1.3 & 8.8 & 10.7 & 20.2 & 26.1 & 38.4 & 58.0 & 24 & 52 \\
1.4 & 6.5 & 16.0 & 23.3 & 37.0 & 43.8 & 49.4 & 32 & 43 \\
1.5 & 4.1 & 15.0 & 12.6 & 33.4 & 43.5 & 45.6 & 30 & 40 \\
1.6 & 0.4 & 15.7 & 11.1 & 36.6 & 44.2 & 46.6 & 32 & 41 \\
1.7 & 3.0 & 9.9 & 20.0 & 29.2 & 38.5 & 42.4 & 28 & 34 \\
1.8 & 8.3 & 14.8 & 12.9 & 30.0 & 38.3 & 43.7 & 28 & 34 \\
1.9 & 2.1 & 4.1 & 8.4 & 43.8 & 48.4 & 49.1 & 36 & 46 \\
2.0 & 1.5 & 13.8 & 15.5 & 48.5 & 49.6 & 56.8 & 39 & 50 \\
\hline
\end{tabular}

Tabla A.9: Parámetros obtenidos en la comparación de la potencia para el nuevo gráfico $D S-U: u_{0}=1, \alpha_{t}=0.05, n=40$ 
APÉNDICE A. COMPARACIÓN DE LA POTENCIA DEL GRÁFICO U FRENTE A LA POTENCIA DEL GRÁFICO DS-U EN UN VALOR U

\begin{tabular}{||c||c|c||c|c|}
\hline$u_{1}$ & Pot $_{U}$ & Pot $_{D S-U}$ & Pot $_{D S-U}-$ Pot $_{U}$ & $\alpha_{\text {real }}$ \\
\hline 0.1 & 1 & 1 & $-2.626969 \cdot 10^{-9}$ & 0.043024 \\
0.2 & 1 & 0.999999 & $-8.124138 \cdot 10^{-7}$ & 0.041382 \\
0.3 & 0.999944 & 0.999999 & $5.527655 \cdot 10^{-5}$ & 0.051253 \\
0.4 & 0.995895 & 0.999807 & $3.912010 \cdot 10^{-3}$ & 0.054957 \\
0.5 & 0.947519 & 0.941764 & $-5.755309 \cdot 10^{-3}$ & 0.056719 \\
0.6 & 0.767742 & 0.837705 & 0.069963 & 0.052457 \\
0.7 & 0.474881 & 0.634554 & 0.159673 & 0.056472 \\
0.8 & 0.216646 & 0.375528 & 0.158882 & 0.056872 \\
0.9 & 0.078269 & 0.142630 & 0.064362 & 0.056859 \\
\hline 1.0 & 0.047396 & 0.056875 & $9.478650 \cdot 10^{-3}$ & 0.056875 \\
\hline 1.1 & 0.106533 & 0.206219 & 0.099686 & 0.056706 \\
1.2 & 0.254211 & 0.457415 & 0.203204 & 0.056873 \\
1.3 & 0.463311 & 0.704689 & 0.241377 & 0.056784 \\
1.4 & 0.673712 & 0.868982 & 0.195271 & 0.055279 \\
1.5 & 0.833356 & 0.951494 & 0.118138 & 0.056897 \\
1.6 & 0.928208 & 0.982825 & 0.054617 & 0.054628 \\
1.7 & 0.973672 & 0.995819 & 0.022147 & 0.056265 \\
1.8 & 0.991689 & 0.998859 & $7.169504 \cdot 10^{-3}$ & 0.048051 \\
1.9 & 0.997716 & 0.999329 & $1.613051 \cdot 10^{-3}$ & 0.047909 \\
2.0 & 0.999447 & 0.999823 & $3.761487 \cdot 10^{-4}$ & 0.053707 \\
\hline
\end{tabular}

Tabla A.10: Resultados para $u_{0}=1, \alpha_{\text {teorico }}=0.05$ y $n=40$ 
APÉNDICE A. COMPARACIÓN DE LA POTENCIA DEL GRÁFICO U FRENTE A LA POTENCIA DEL GRÁFICO DS-U EN UN VALOR U

A.6. Comparación de los gráficos de control $u$ y $\boldsymbol{D S}-\boldsymbol{U} \operatorname{con} n=40, u_{0}=1$ y $\alpha_{t}=0.0027$ 
APÉNDICE A. COMPARACIÓN DE LA POTENCIA DEL GRÁFICO U FRENTE A LA POTENCIA DEL GRÁFICO DS-U EN UN VALOR U

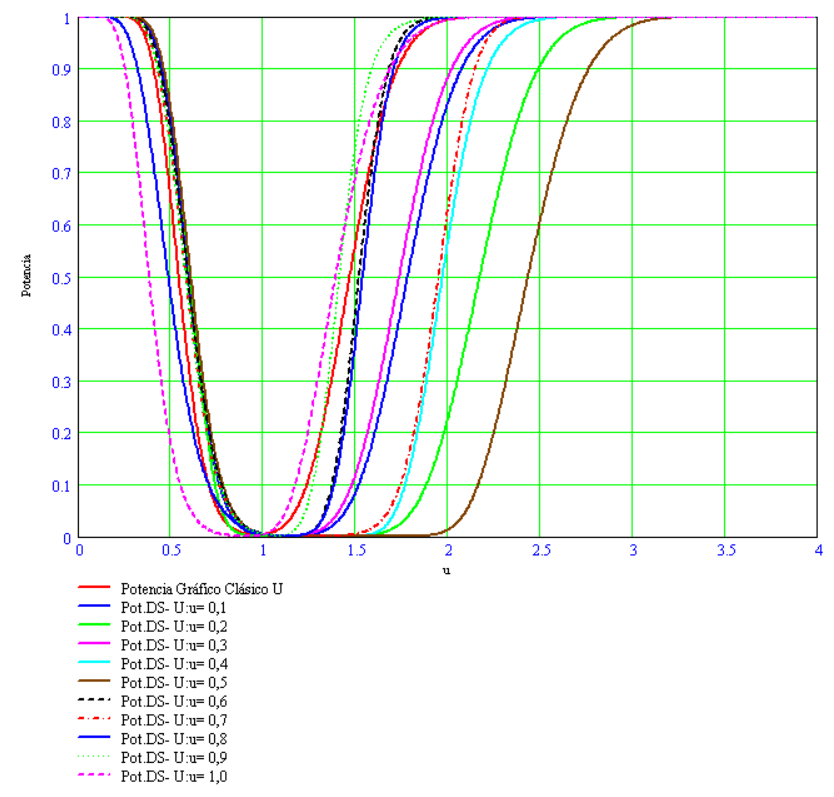

(a) $u_{1} \in[0.1,1.0]$
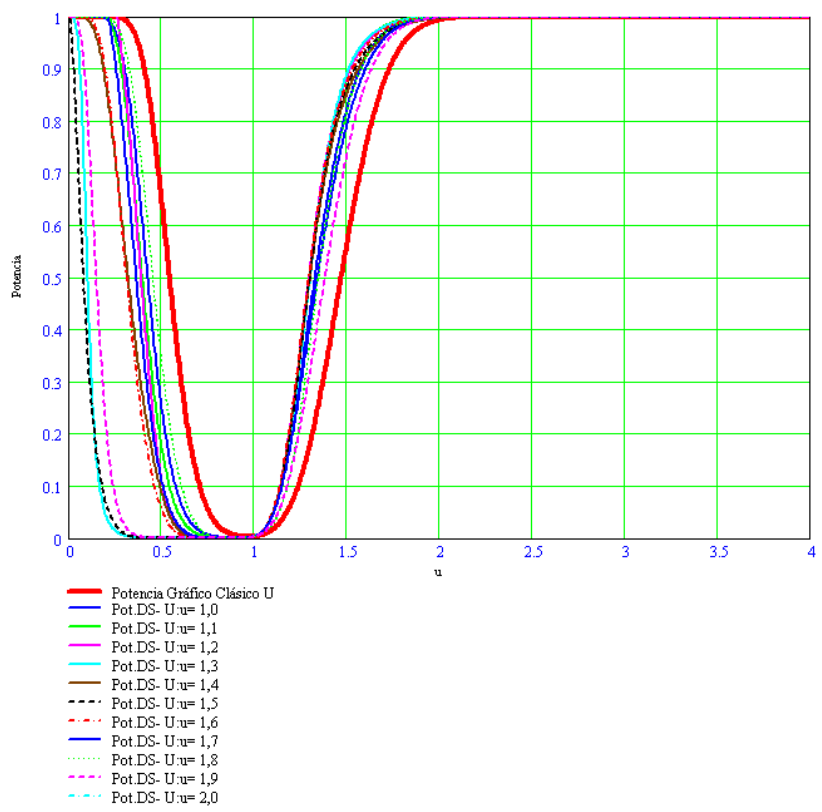

(b) $u_{1} \in[1.1,2.0]$

Fig. A.116: Curvas de potencia en distintos valores de $u_{1}$ 
APÉNDICE A. COMPARACIÓN DE LA POTENCIA DEL GRÁFICO U FRENTE A LA POTENCIA DEL GRÁFICO DS-U EN UN VALOR U

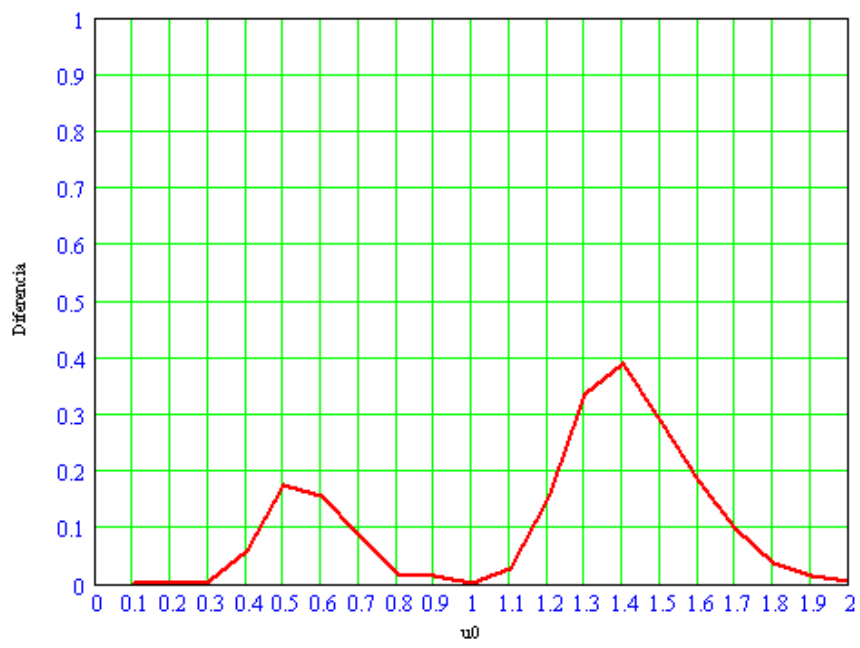

Fig. A.117: $\operatorname{Pot}_{D S-U}-\operatorname{Pot}_{u}$ para $n=40, u_{0}=1$ y $\alpha_{t}=0.0027$

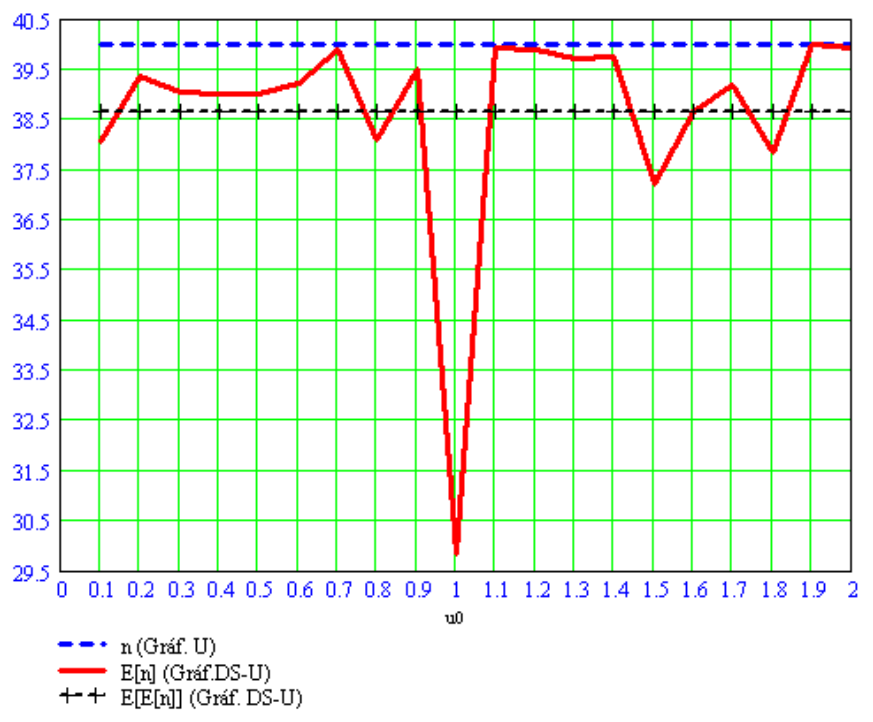

Fig. A.118: Tamaño medio de muestra en el gráfico $D S-U$ para $n=40, u_{0}=1$ y $\alpha_{t}=0.0027$ 
APÉNDICE A. COMPARACIÓN DE LA POTENCIA DEL GRÁFICO U FRENTE A LA POTENCIA DEL GRÁFICO DS-U EN UN VALOR U

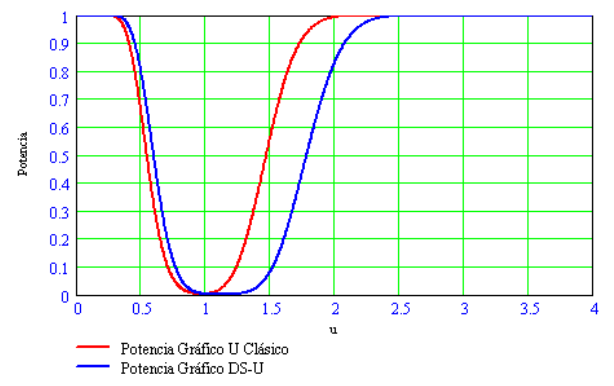

Fig. A.119: Curvas de potencia para $u_{1}=0,1$

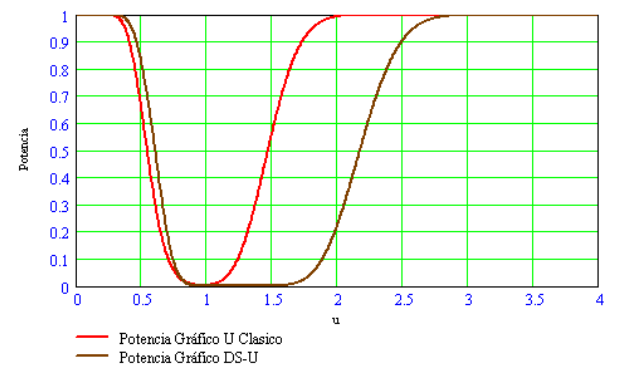

Fig. A.120: Curvas de potencia para $u_{1}=0,2$

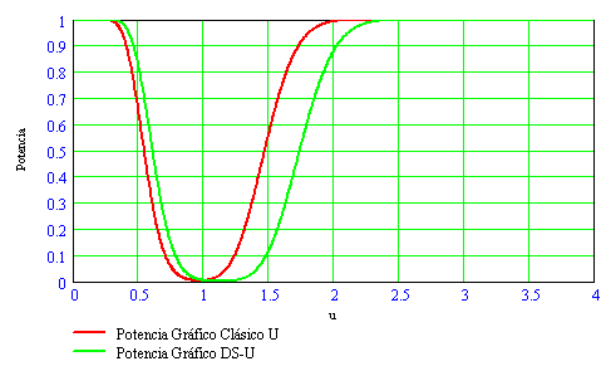

Fig. A.121: Curvas de potencia para $u_{1}=0,3$ 
APÉNDICE A. COMPARACIÓN DE LA POTENCIA DEL GRÁFICO U FRENTE A LA POTENCIA DEL GRÁFICO DS-U EN UN VALOR U

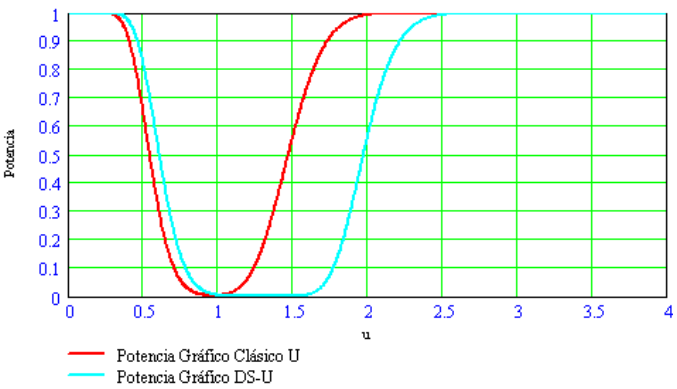

Fig. A.122: Curvas de potencia para $u_{1}=0,4$

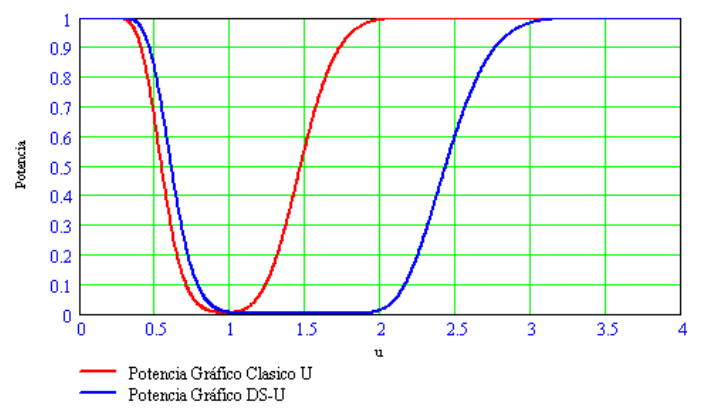

Fig. A.123: Curvas de potencia para $u_{1}=0,5$

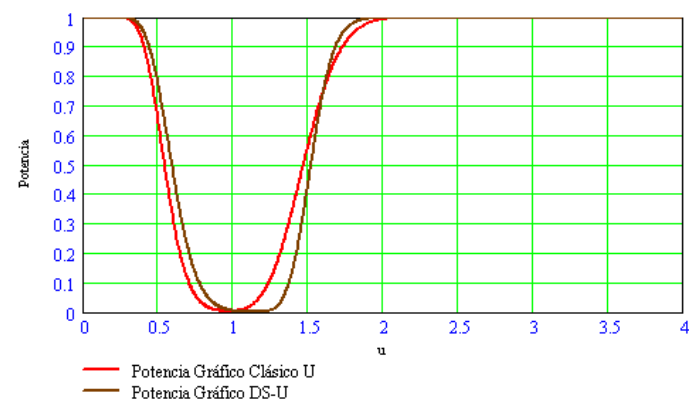

Fig. A.124: Curvas de potencia para $u_{1}=0,6$ 
APÉNDICE A. COMPARACIÓN DE LA POTENCIA DEL GRÁFICO U FRENTE A LA POTENCIA DEL GRÁFICO DS-U EN UN VALOR U

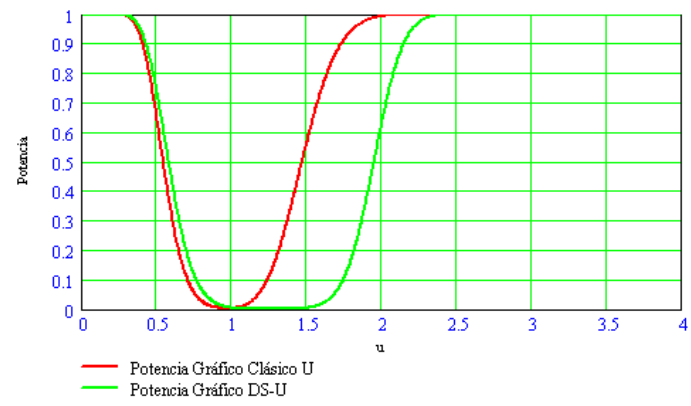

Fig. A.125: Curvas de potencia para $u_{1}=0,7$

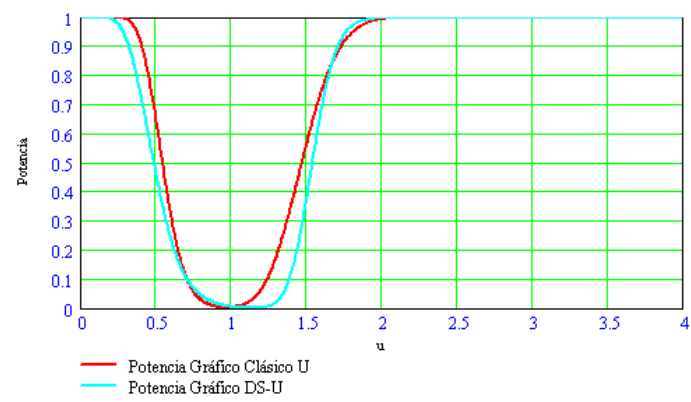

Fig. A.126: Curvas de potencia para $u_{1}=0,8$

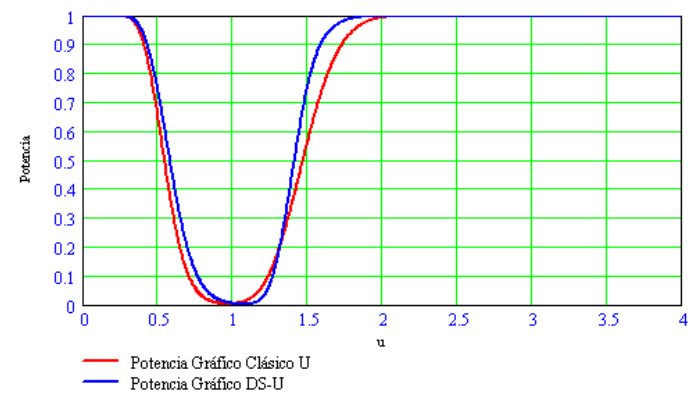

Fig. A.127: Curvas de potencia para $u_{1}=0,9$ 
APÉNDICE A. COMPARACIÓN DE LA POTENCIA DEL GRÁFICO U FRENTE A LA POTENCIA DEL GRÁFICO DS-U EN UN VALOR U

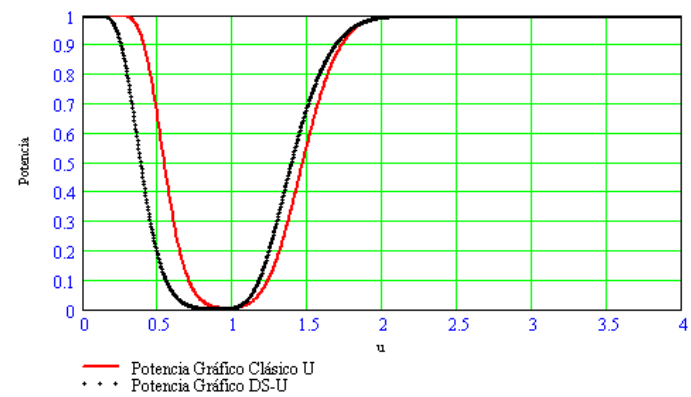

Fig. A.128: Curvas de potencia para $u_{1}=1,0$

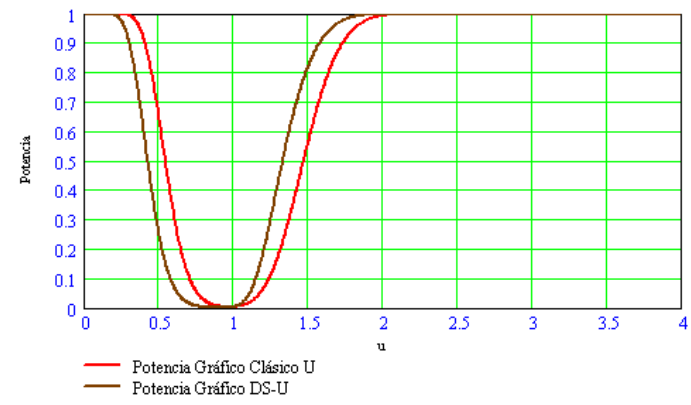

Fig. A.129: Curvas de potencia para $u_{1}=1,1$

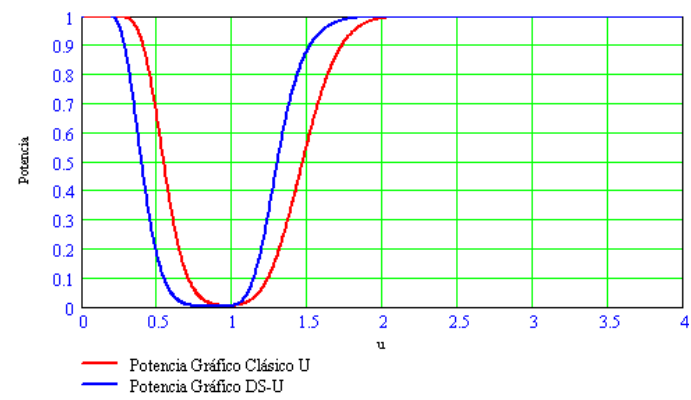

Fig. A.130: Curvas de potencia para $u_{1}=1,2$ 
APÉNDICE A. COMPARACIÓN DE LA POTENCIA DEL GRÁFICO U FRENTE A LA POTENCIA DEL GRÁFICO DS-U EN UN VALOR U

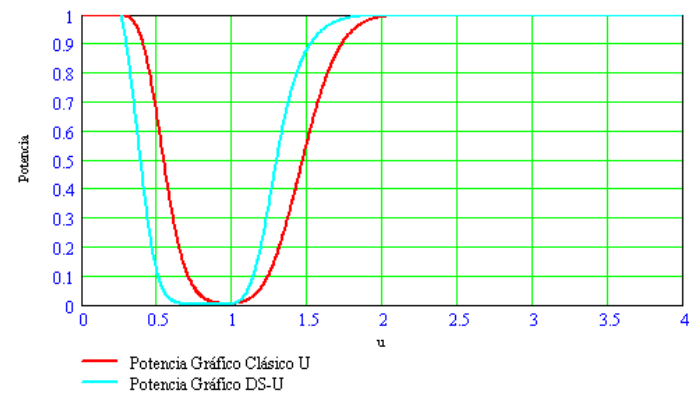

Fig. A.131: Curvas de potencia para $u_{1}=1,3$

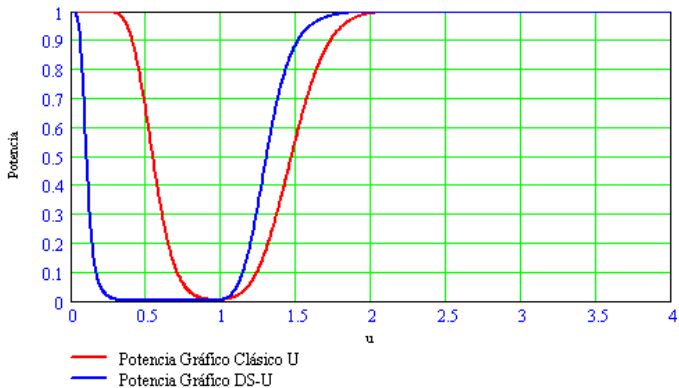

Fig. A.132: Curvas de potencia para $u_{1}=1,4$

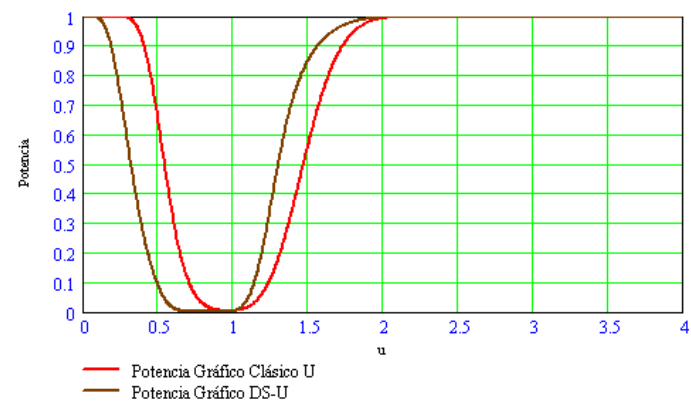

Fig. A.133: Curvas de potencia para $u_{1}=1,5$ 
APÉNDICE A. COMPARACIÓN DE LA POTENCIA DEL GRÁFICO U FRENTE A LA POTENCIA DEL GRÁFICO DS-U EN UN VALOR U

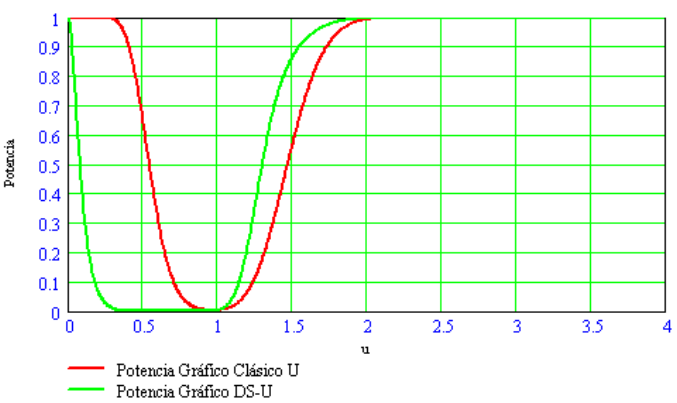

Fig. A.134: Curvas de potencia para $u_{1}=1,6$

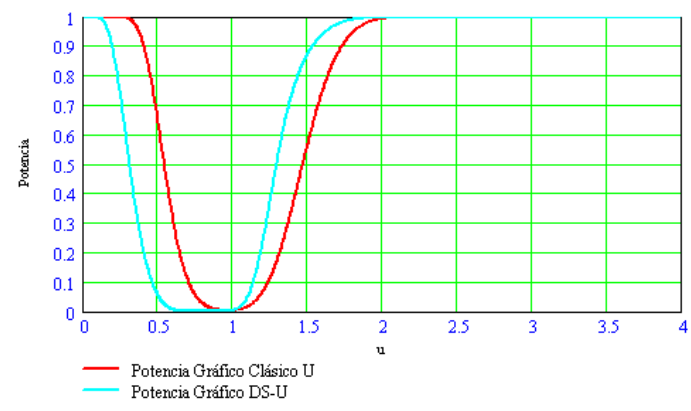

Fig. A.135: Curvas de potencia para $u_{1}=1,7$

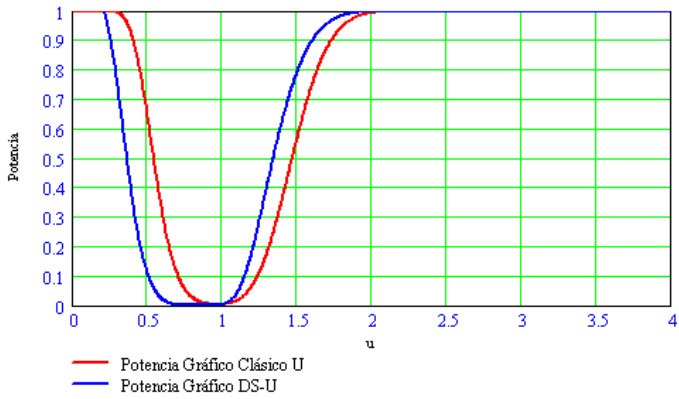

Fig. A.136: Curvas de potencia para $u_{1}=1,8$ 
APÉNDICE A. COMPARACIÓN DE LA POTENCIA DEL GRÁFICO U FRENTE A LA POTENCIA DEL GRÁFICO DS-U EN UN VALOR U

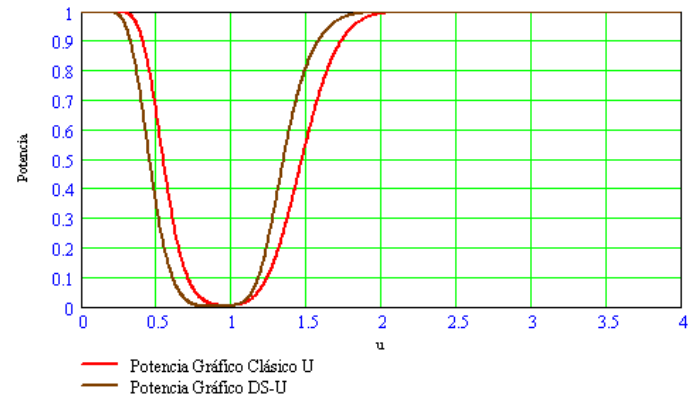

Fig. A.137: Curvas de potencia para $u_{1}=1,9$

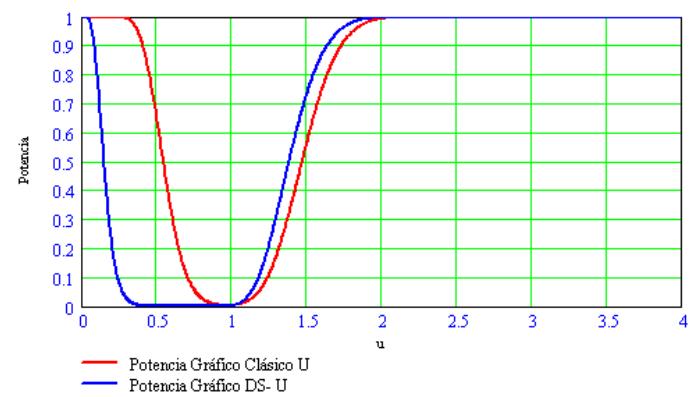

Fig. A.138: Curvas de potencia para $u_{1}=2,0$ 
APÉNDICE A. COMPARACIÓN DE LA POTENCIA DEL GRÁFICO U FRENTE A LA POTENCIA DEL GRÁFICO DS-U EN UN VALOR U

\begin{tabular}{|c||c|c|c|c|c|c||c|c|}
\hline$u_{1}$ & $L C I$ & $L A I$ & $L C I_{1}$ & $L A S$ & $L C S$ & $L C S_{1}$ & $n_{1}$ & $n_{2}$ \\
\hline 0.1 & 11.8 & 20.6 & 34.3 & 93.2 & 154.2 & 157.1 & 35 & 61 \\
0.2 & 18.1 & 23.3 & 71.5 & 84.2 & 128.3 & 126.5 & 39 & 95 \\
0.3 & 11.2 & 23.8 & 70.0 & 68.0 & 83.3 & 155.8 & 39 & 12 \\
0.4 & 23.1 & 23.6 & 43.5 & 73.2 & 118.2 & 113.8 & 39 & 62 \\
0.5 & 19.1 & 19.2 & 33.9 & 41.6 & 61.6 & 111.5 & 34 & 56 \\
0.6 & 20.1 & 20.2 & 71.0 & 45.4 & 70.4 & 147.1 & 35 & 99 \\
0.7 & 19.4 & 19.9 & 56.1 & 43 & 70.8 & 156.2 & 34 & 77 \\
0.8 & 10.2 & 10.3 & 72 & 26.6 & 43 & 146.8 & 22 & 96 \\
0.9 & 19.2 & 19.7 & 75.7 & 43.1 & 58.2 & 133.6 & 34 & 98 \\
\hline 1.0 & 10.1 & 10.3 & 44.6 & 38.2 & 51.3 & 73.7 & 28 & 65 \\
\hline 1.1 & 15.3 & 15.5 & 63.7 & 48.7 & 58.9 & 99.3 & 37 & 88 \\
1.2 & 11.0 & 11.2 & 52.4 & 39.2 & 51.4 & 99.2 & 32 & 83 \\
1.3 & 8.0 & 10.7 & 47.0 & 35.7 & 47.8 & 111.6 & 29 & 93 \\
1.4 & 1.5 & 3.6 & 8 & 29.2 & 44.1 & 99.1 & 25 & 81 \\
1.5 & 1.4 & 6.3 & 57.3 & 25.3 & 37.5 & 120 & 21 & 100 \\
1.6 & 0.2 & 1.9 & 33.1 & 26.1 & 37.3 & 121.9 & 22 & 100 \\
1.7 & 2.4 & 8.5 & 58.6 & 34.2 & 47 & 118.2 & 28 & 100 \\
1.8 & 3 & 12.2 & 63.6 & 46.1 & 140.8 & 104.1 & 35 & 94 \\
1.9 & 2.8 & 16.6 & 74.7 & 48.8 & 54.1 & 121 & 37 & 98 \\
2.0 & 3.7 & 5 & 42 & 53.9 & 144.5 & 74.3 & 39 & 69 \\
\hline
\end{tabular}

Tabla A.11: Parámetros obtenidos en la comparación de la potencia para el nuevo gráfico $D S-U: u_{0}=1, \alpha=0.0027, n=40$. 
APÉNDICE A. COMPARACIÓN DE LA POTENCIA DEL GRÁFICO U FRENTE A LA POTENCIA DEL GRÁFICO DS-U EN UN VALOR U

\begin{tabular}{|c||c|c||c|c|}
\hline$u_{1}$ & Pot $_{U}$ & Pot $_{D S-U}$ & Pot $_{D S-U}-$ Pot $_{U}$ & $\alpha_{\text {real }}$ \\
\hline 0.1 & 1 & 1 & $3.241928 \cdot 10^{-10}$ & $2.010688 \cdot 10^{-3}$ \\
0.2 & 0.999967 & 0.999998 & $3.095655 \cdot 10^{-5}$ & $1.684322 \cdot 10^{-4}$ \\
0.3 & 0.993935 & 0.998939 & $5.004302 \cdot 10^{-3}$ & $4.008770 \cdot 10^{-3}$ \\
0.4 & 0.910773 & 1.061791 & 0.151017 & $4.028716 \cdot 10^{-3}$ \\
0.5 & 0.643698 & 0.819634 & 0.175936 & $3.992446 \cdot 10^{-3}$ \\
0.6 & 0.313928 & 0.470976 & 0.157048 & $4.353411 \cdot 10^{-3}$ \\
0.7 & 0.105986 & 0.212756 & 0.106769 & $4.297589 \cdot 10^{-3}$ \\
0.8 & 0.026008 & 0.043076 & 0.017068 & $4.346166 \cdot 10^{-3}$ \\
0.9 & $5.150110 \cdot 10^{-3}$ & 0.018197 & 0.013047 & $4.353710 \cdot 10^{-3}$ \\
\hline 1.0 & $3.631613 \cdot 10^{-3}$ & $4.357052 \cdot 10^{-3}$ & $7.254397 \cdot 10^{-4}$ & $4.357052 \cdot 10^{-3}$ \\
\hline 1.1 & 0.017787 & 0.046081 & 0.028294 & $4.355361 \cdot 10^{-3}$ \\
1.2 & 0.068373 & 0.219109 & 0.150735 & $4.356488 \cdot 10^{-3}$ \\
1.3 & 0.182409 & 0.518135 & 0.335726 & $4.255065 \cdot 10^{-3}$ \\
1.4 & 0.361748 & 0.751211 & 0.389463 & $4.320541 \cdot 10^{-3}$ \\
1.5 & 0.568601 & 0.854150 & 0.285549 & $4.205839 \cdot 10^{-3}$ \\
1.6 & 0.750648 & 0.933315 & 0.182666 & $4.221679 \cdot 10^{-3}$ \\
1.7 & 0.876936 & 0.975697 & 0.098761 & $4.267294 \cdot 10^{-3}$ \\
1.8 & 0.947886 & 0.984578 & 0.036692 & $4.251739 \cdot 10^{-3}$ \\
1.9 & 0.980911 & 0.996875 & 0.015964 & $3.881520 \cdot 10^{-3}$ \\
2.0 & 0.993895 & 0.998263 & $4.367733 \cdot 10^{-3}$ & $3.293413 \cdot 10^{-3}$ \\
\hline
\end{tabular}

Tabla A.12: Resultados para $u_{0}=1, \alpha_{\text {teórico }}=0.0027$ y $n=40$ 
APÉNDICE A. COMPARACIÓN DE LA POTENCIA DEL GRÁFICO U FRENTE A LA POTENCIA DEL GRÁFICO DS-U EN UN VALOR U

A.7. Comparación de los gráficos de control $u$ y $\boldsymbol{D S}-\boldsymbol{U} \operatorname{con} n=8, u_{0}=5$ y $\alpha_{t}=0.01$ 
APÉNDICE A. COMPARACIÓN DE LA POTENCIA DEL GRÁFICO U FRENTE A LA POTENCIA DEL GRÁFICO DS-U EN UN VALOR U

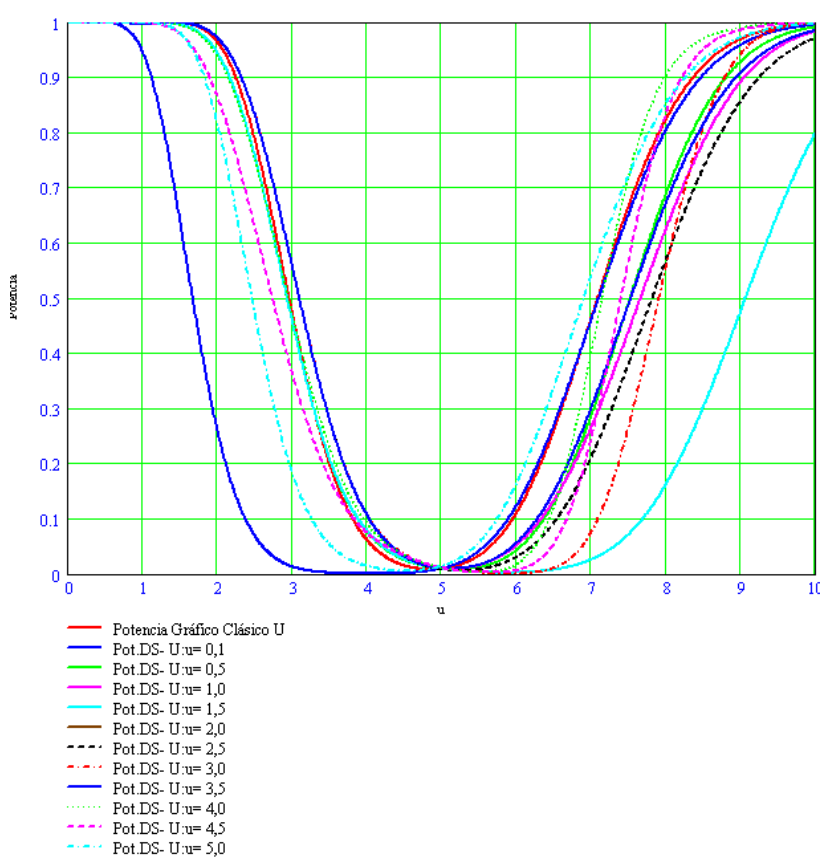

(a) $u_{1} \in[0.1,5.0]$

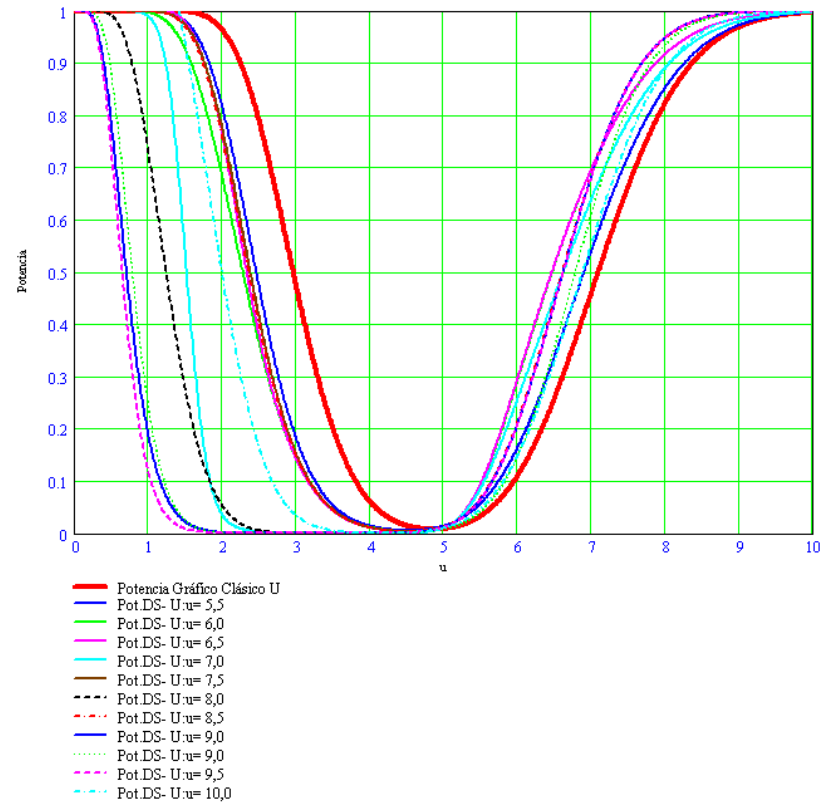

(b) $u_{1} \in[5.5,10.0]$

Fig. A.139: Curvas de potencia en distintos valores de $u_{1}$ 
APÉNDICE A. COMPARACIÓN DE LA POTENCIA DEL GRÁFICO U FRENTE A LA POTENCIA DEL GRÁFICO DS-U EN UN VALOR U

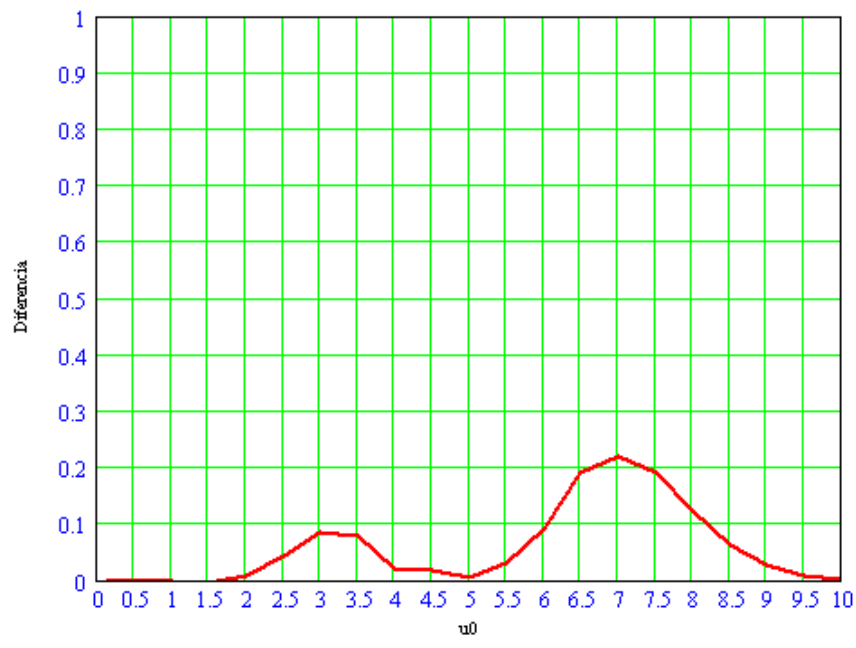

Fig. A.140: $\operatorname{Pot}_{D S-U}-$ Pot $_{u}$ para $u_{0}=5, n=8$ y $\alpha_{t}=0.01$

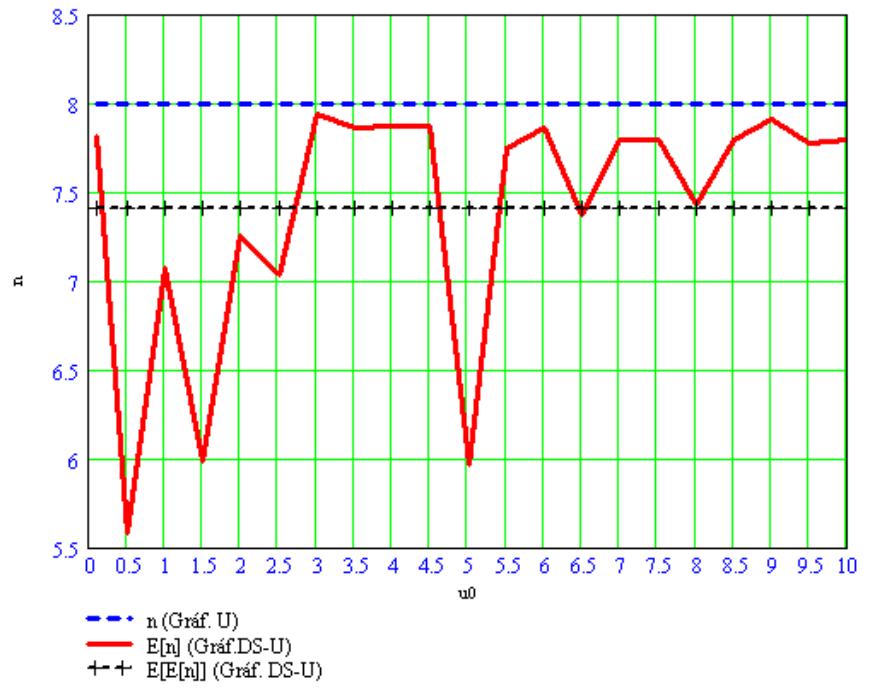

Fig. A.141: Tamaño medio de muestra en el gráfico $D S-U$ para $u_{0}=5, n=8$ y $\alpha_{t}=0.01$ 
APÉNDICE A. COMPARACIÓN DE LA POTENCIA DEL GRÁFICO U FRENTE A LA POTENCIA DEL GRÁFICO DS-U EN UN VALOR U

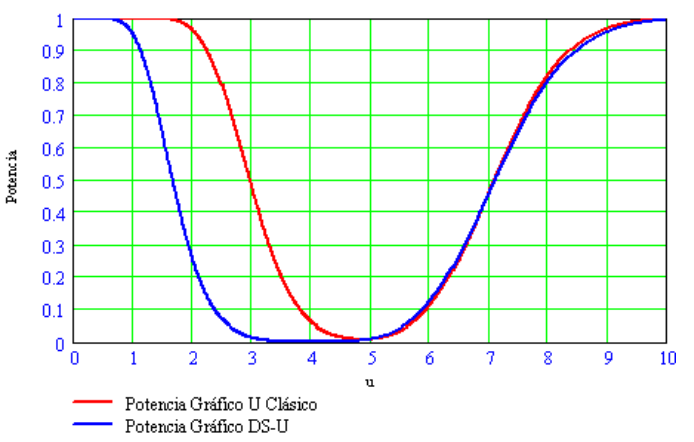

Fig. A.142: Curvas de potencia para $u_{1}=0,1$

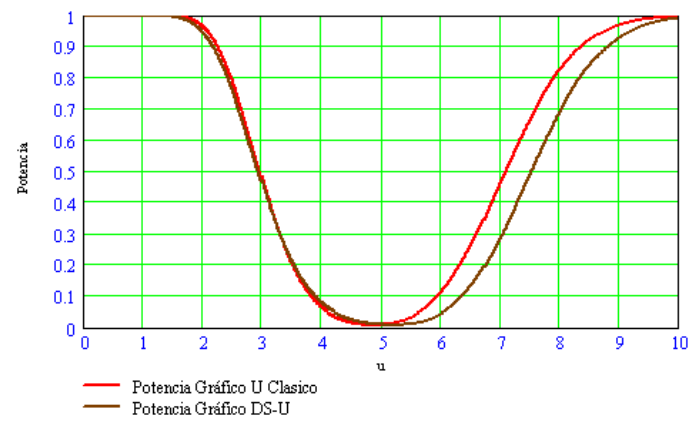

Fig. A.143: Curvas de potencia para $u_{1}=0,5$

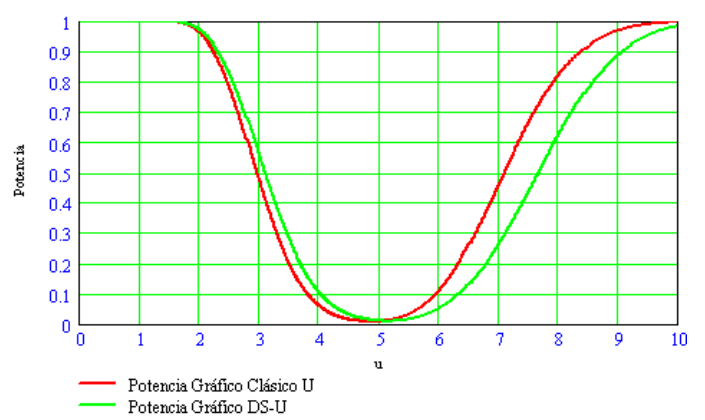

Fig. A.144: Curvas de potencia para $u_{1}=1,0$ 
APÉNDICE A. COMPARACIÓN DE LA POTENCIA DEL GRÁFICO U FRENTE A LA POTENCIA DEL GRÁFICO DS-U EN UN VALOR U

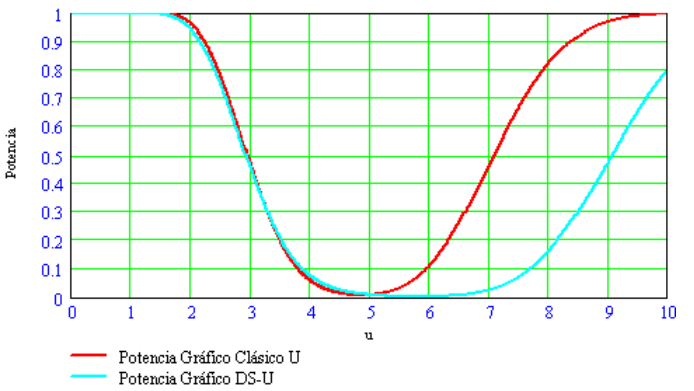

Fig. A.145: Curvas de potencia para $u_{1}=1,5$

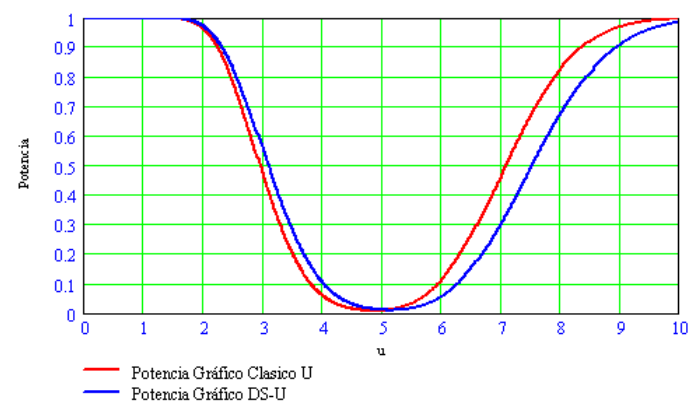

Fig. A.146: Curvas de potencia para $u_{1}=2,0$

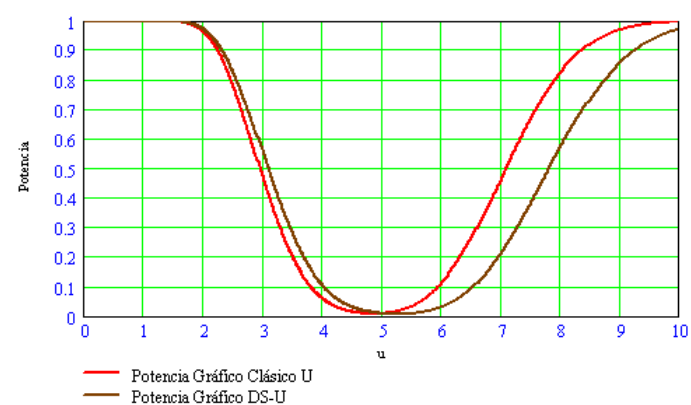

Fig. A.147: Curvas de potencia para $u_{1}=2,5$ 
APÉNDICE A. COMPARACIÓN DE LA POTENCIA DEL GRÁFICO U FRENTE A LA POTENCIA DEL GRÁFICO DS-U EN UN VALOR U

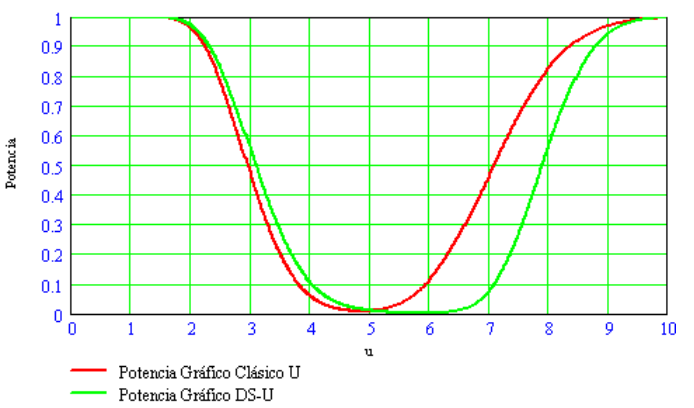

Fig. A.148: Curvas de potencia para $u_{1}=3,0$

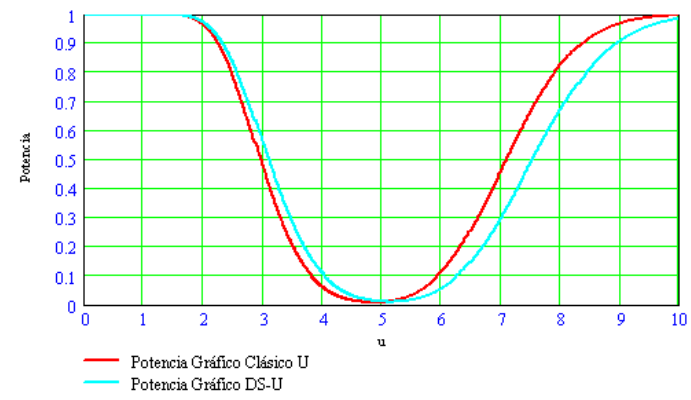

Fig. A.149: Curvas de potencia para $u_{1}=3,5$

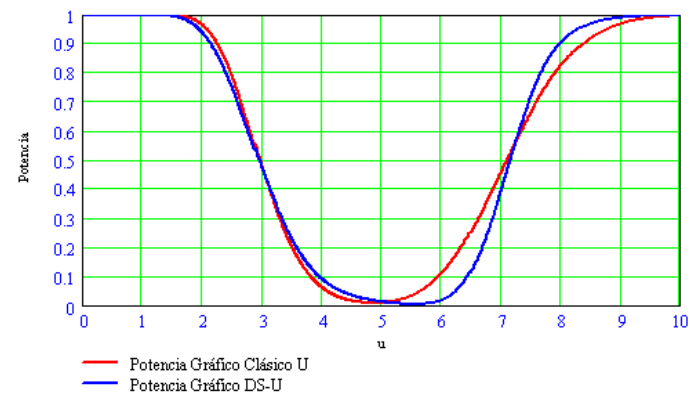

Fig. A.150: Curvas de potencia para $u_{1}=4,0$ 
APÉNDICE A. COMPARACIÓN DE LA POTENCIA DEL GRÁFICO U FRENTE A LA POTENCIA DEL GRÁFICO DS-U EN UN VALOR U

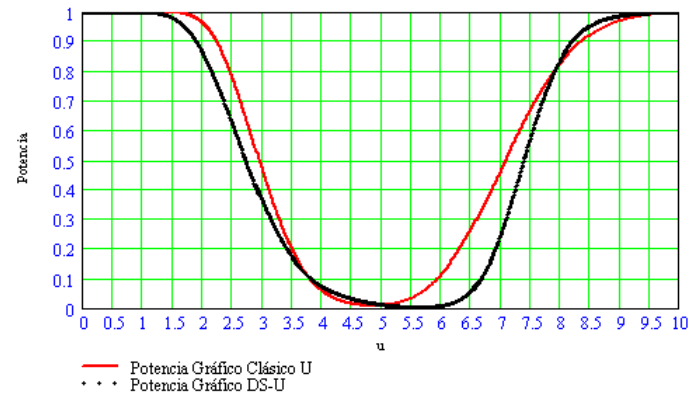

Fig. A.151: Curvas de potencia para $u_{1}=4,5$

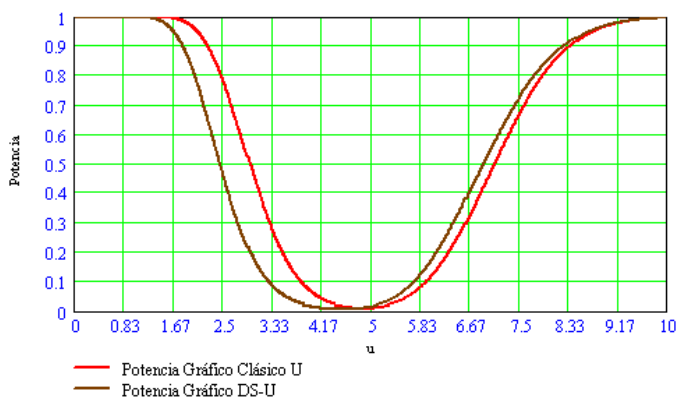

Fig. A.152: Curvas de potencia para $u_{1}=5,0$

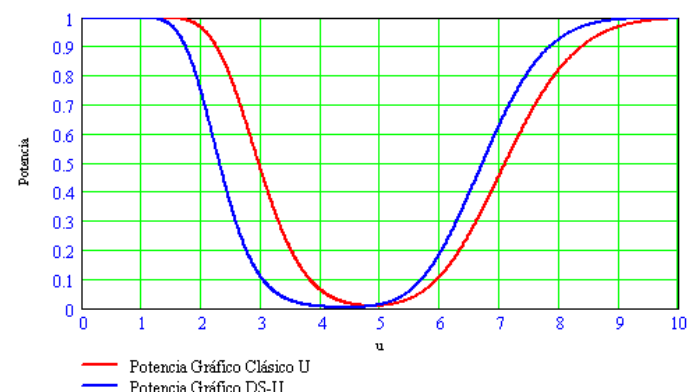

Fig. A.153: Curvas de potencia para $u_{1}=5,5$ 
APÉNDICE A. COMPARACIÓN DE LA POTENCIA DEL GRÁFICO U FRENTE A LA POTENCIA DEL GRÁFICO DS-U EN UN VALOR U

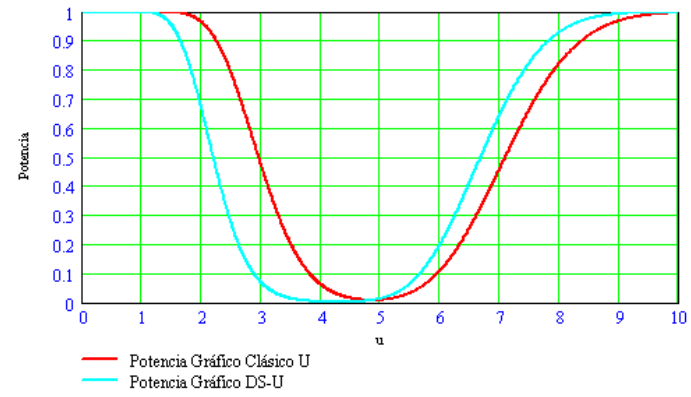

Fig. A.154: Curvas de potencia para $u_{1}=6,0$

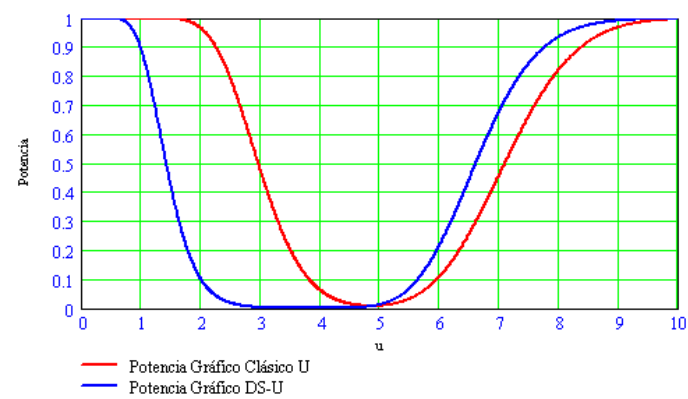

Fig. A.155: Curvas de potencia para $u_{1}=6,5$

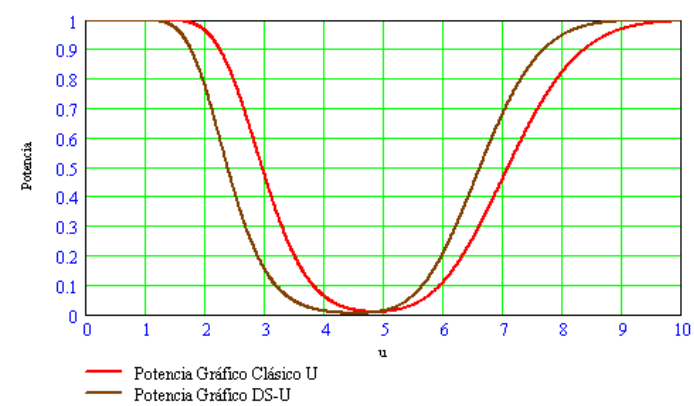

Fig. A.156: Curvas de potencia para $u_{1}=7,0$ 
APÉNDICE A. COMPARACIÓN DE LA POTENCIA DEL GRÁFICO U FRENTE A LA POTENCIA DEL GRÁFICO DS-U EN UN VALOR U

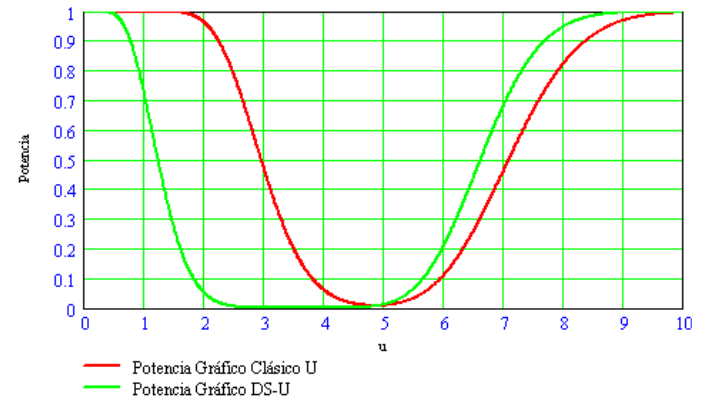

Fig. A.157: Curvas de potencia para $u_{1}=7,5$

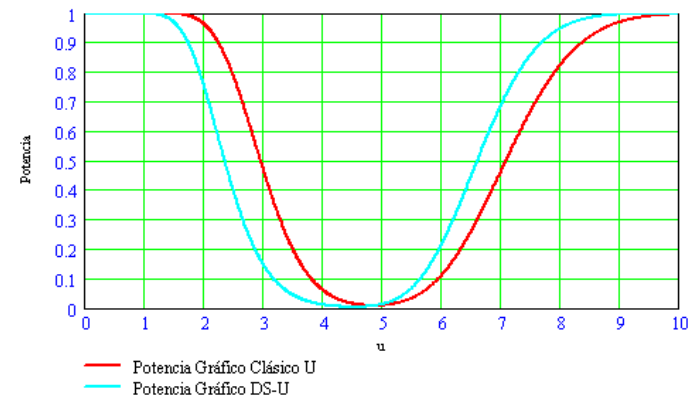

Fig. A.158: Curvas de potencia para $u_{1}=8,0$

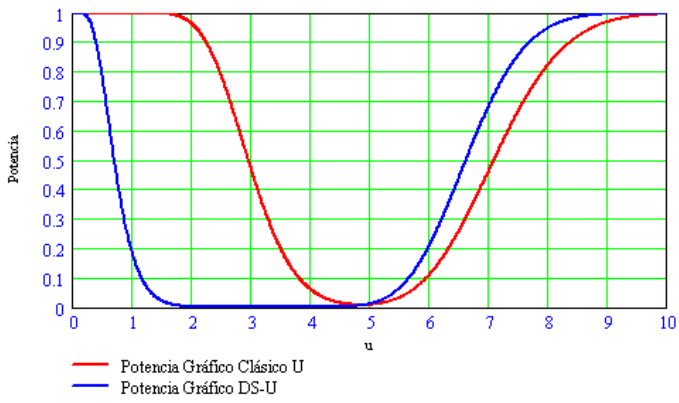

Fig. A.159: Curvas de potencia para $u_{1}=8,5$ 
APÉNDICE A. COMPARACIÓN DE LA POTENCIA DEL GRÁFICO U FRENTE A LA POTENCIA DEL GRÁFICO DS-U EN UN VALOR U

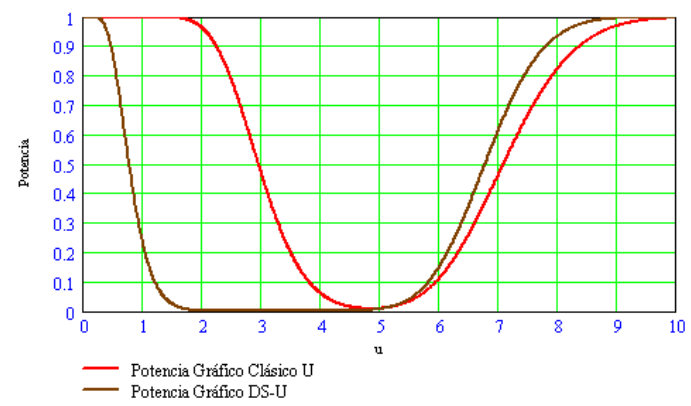

Fig. A.160: Curvas de potencia para $u_{1}=9,0$

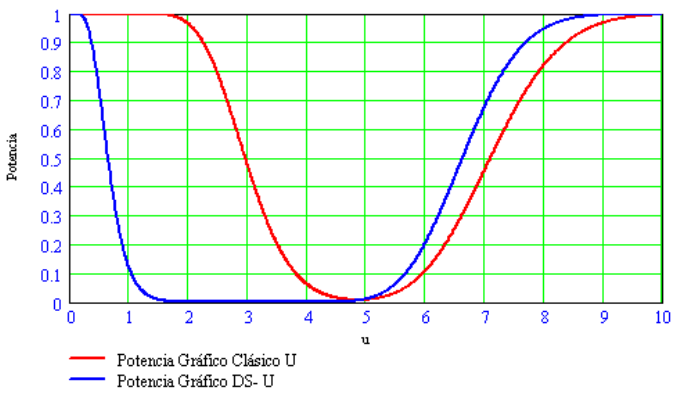

Fig. A.161: Curvas de potencia para $u_{1}=9,5$

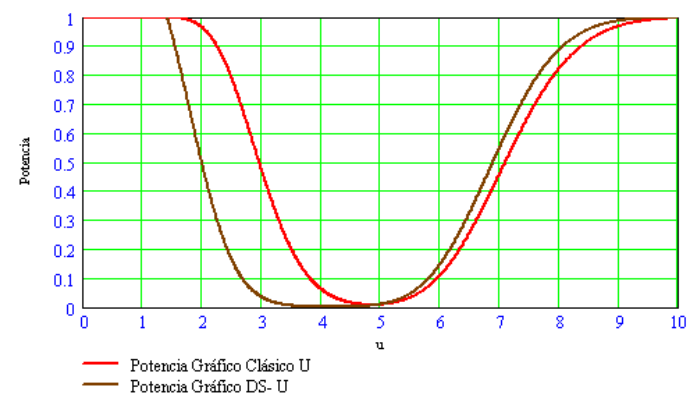

Fig. A.162: Curvas de potencia para $u_{1}=10,0$ 
APÉNDICE A. COMPARACIÓN DE LA POTENCIA DEL GRÁFICO U FRENTE A LA POTENCIA DEL GRÁFICO DS-U EN UN VALOR U

\begin{tabular}{|c||c|c|c|c|c|c||c|c|}
\hline$u_{1}$ & $L C I$ & $L A I$ & $L C I_{1}$ & $L A S$ & $L C S$ & $L C S_{1}$ & $n_{1}$ & $n_{2}$ \\
\hline 0.1 & 6.9 & 8.8 & 7.2 & 37.2 & 45.0 & 38.3 & 5 & 21 \\
0.5 & 13.4 & 15.0 & 15.0 & 31.3 & 43.3 & 37.7 & 5 & 5 \\
1.0 & 19.3 & 21.3 & 78.0 & 52.6 & 53.4 & 113.6 & 7 & 12 \\
1.5 & 13.8 & 15.0 & 15.0 & 29.8 & 47.2 & 50.5 & 5 & 5 \\
2.0 & 21.1 & 21.7 & 24.8 & 52.2 & 93.9 & 76.7 & 7 & 92 \\
2.5 & 21.3 & 21.5 & 29.8 & 54.6 & 149.9 & 138.2 & 7 & 31 \\
3.0 & 21.1 & 21.6 & 65.7 & 44.1 & 70.3 & 125.8 & 7 & 16 \\
3.5 & 13.6 & 21.9 & 50.9 & 52.7 & 150.1 & 79.3 & 7 & 84 \\
4.0 & 13.2 & 15.0 & 13.8 & 25.0 & 45.6 & 40.5 & 5 & 5 \\
4.5 & 10.5 & 21.1 & 11.8 & 52.5 & 150.0 & 151.5 & 7 & 52 \\
\hline 5.0 & 10.2 & 14.9 & 11.4 & 29.5 & 49.7 & 32.3 & 5 & 5 \\
\hline 5.5 & 9.8 & 15.0 & 15.0 & 27.0 & 41.5 & 46.5 & 5 & 7 \\
6.0 & 8.9 & 14.8 & 15.0 & 27.9 & 42.9 & 45.9 & 5 & 7 \\
6.5 & 5.8 & 12.9 & 9.5 & 29.9 & 42.4 & 49.2 & 5 & 8 \\
7.0 & 13.4 & 15.0 & 14.4 & 33.9 & 47.0 & 45.7 & 6 & 7 \\
7.5 & 0.5 & 15.0 & 9.0 & 33.6 & 47.6 & 45.7 & 6 & 7 \\
8.0 & 13.1 & 15.0 & 13.8 & 34.7 & 48.9 & 44.9 & 6 & 7 \\
8.5 & 1.3 & 15.0 & 4.9 & 33.9 & 47.1 & 45.6 & 6 & 7 \\
9.0 & 3.1 & 13.9 & 4.9 & 41.1 & 50.4 & 49.6 & 7 & 7 \\
9.5 & 2.4 & 7.5 & 3.1 & 33.6 & 47.0 & 45.5 & 6 & 7 \\
10.0 & 5.0 & 15.0 & 11.7 & 25.1 & 39.6 & 42.3 & 5 & 6 \\
\hline
\end{tabular}

Tabla A.13: Parámetros obtenidos en la comparación de la potencia para el nuevo gráfico $D S-U: u_{0}=5, \alpha_{t}=0.01, n=8$. 
APÉNDICE A. COMPARACIÓN DE LA POTENCIA DEL GRÁFICO U FRENTE A LA POTENCIA DEL GRÁFICO DS-U EN UN VALOR U

\begin{tabular}{|c||c|c||c|c|}
\hline$u_{1}$ & Pot $_{U}$ & Pot $_{D S-U}$ & Pot $_{D S-U}-$ Pot $_{U}$ & $\alpha_{\text {real }}$ \\
\hline 0.1 & 1 & 1 & $-1.516243 \cdot 10^{-11}$ & $9.847689 \cdot 10^{-3}$ \\
0.5 & 1 & 1 & $-1.068255 \cdot 10^{-8}$ & $9.333230 \cdot 10^{-3}$ \\
1.0 & 0.999996 & 0.999995 & $-8.008246 \cdot 10^{-7}$ & 0.010224 \\
1.5 & 0.998527 & 0.995218 & $-3.309416 \cdot 10^{-3}$ & $8.950808 \cdot 10^{-3}$ \\
2.0 & 0.963314 & 0.971156 & $7.841602 \cdot 10^{-3}$ & 0.010315 \\
2.5 & 0.787493 & 0.831851 & 0.044358 & $8.553644 \cdot 10^{-3}$ \\
3.0 & 0.472850 & 0.557690 & 0.084840 & 0.010449 \\
3.5 & 0.199810 & 0.279426 & 0.079616 & 0.010315 \\
4.0 & 0.061012 & 0.090402 & 0.029391 & 0.010951 \\
4.5 & 0.014796 & 0.031769 & 0.016973 & 0.010315 \\
\hline 5.0 & $9.131637 \cdot 10^{-3}$ & 0.014724 & $5.592575 \cdot 10^{-3}$ & 0.014724 \\
\hline 5.5 & 0.034119 & 0.094667 & 0.060548 & 0.010958 \\
6.0 & 0.111937 & 0.298138 & 0.186201 & 0.010911 \\
6.5 & 0.261682 & 0.455806 & 0.194125 & 0.010784 \\
7.0 & 0.464541 & 0.684286 & 0.219745 & 0.012766 \\
7.5 & 0.668064 & 0.858848 & 0.190784 & 0.012363 \\
8.0 & 0.825224 & 0.948664 & 0.123439 & 0.013196 \\
8.5 & 0.921623 & 0.985603 & 0.063979 & 0.012363 \\
9.0 & 0.969841 & 0.995845 & 0.026004 & $7.817370 \cdot 10^{-3}$ \\
9.5 & 0.989946 & 0.999258 & $9.311412 \cdot 10^{-3}$ & 0.012281 \\
10.0 & 0.997067 & 0.999343 & $2.275568 \cdot 10^{-3}$ & 0.010380 \\
\hline
\end{tabular}

Tabla A.14: Resultados para $u_{0}=5, \alpha_{\text {teórico }}=0.01$ y $n=8$ 
APÉNDICE A. COMPARACIÓN DE LA POTENCIA DEL GRÁFICO U FRENTE A LA POTENCIA DEL GRÁFICO DS-U EN UN VALOR U

A.8. Comparación de los gráficos de control $u$ y $\boldsymbol{D S}$-U con $n=8, u_{0}=5$ y $\alpha_{t}=0.05$ 
APÉNDICE A. COMPARACIÓN DE LA POTENCIA DEL GRÁFICO U FRENTE A LA POTENCIA DEL GRÁFICO DS-U EN UN VALOR U

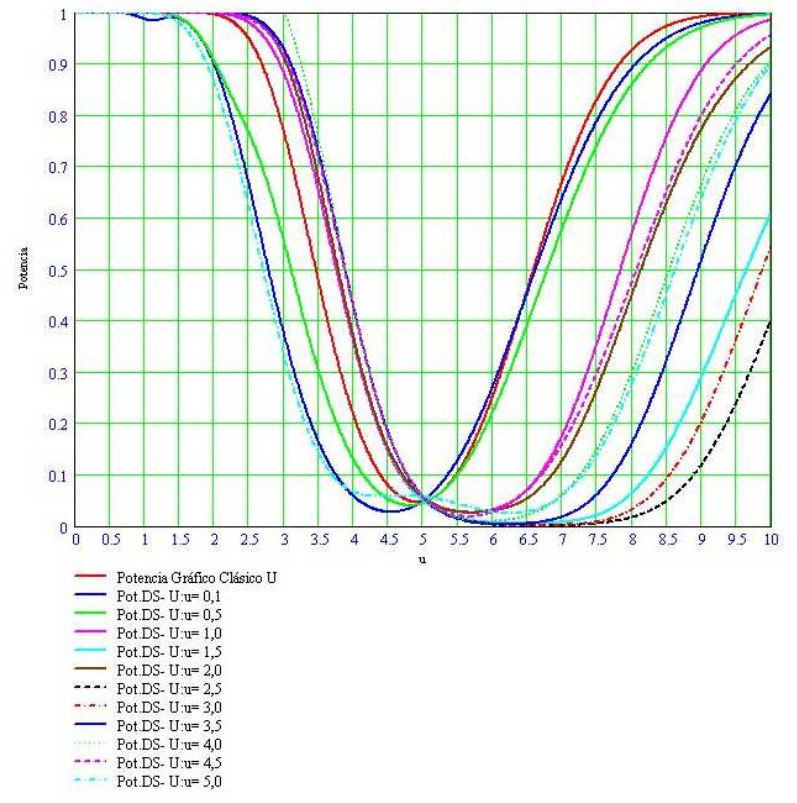

(a) $u_{1} \in[0.1,5.0]$
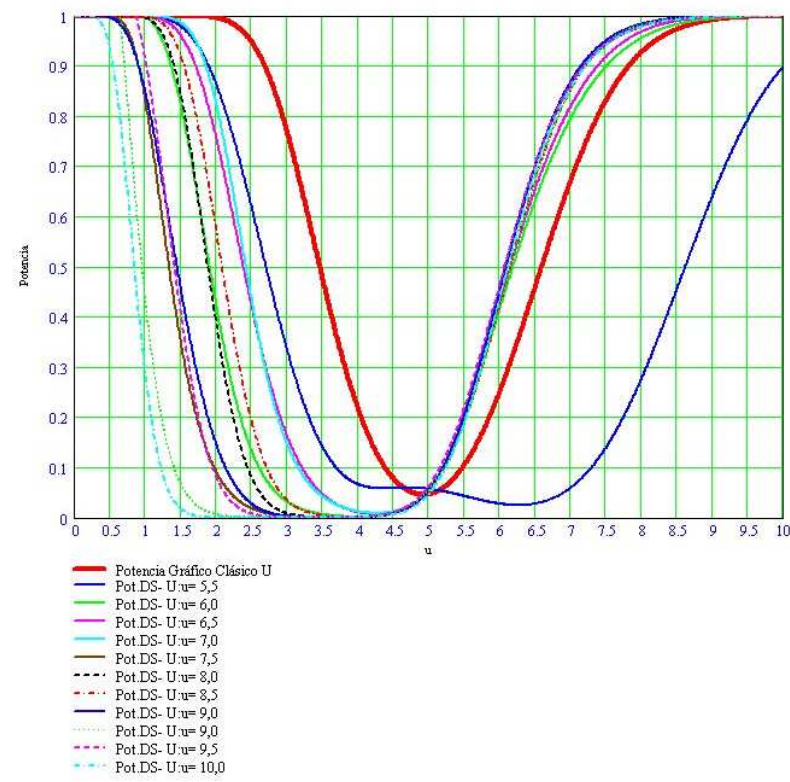

(b) $u_{1} \in[5.5,10.0]$

Fig. A.163: Curvas de potencia en distintos valores de $u_{1}$ 
APÉNDICE A. COMPARACIÓN DE LA POTENCIA DEL GRÁFICO U FRENTE A LA POTENCIA DEL GRÁFICO DS-U EN UN VALOR U

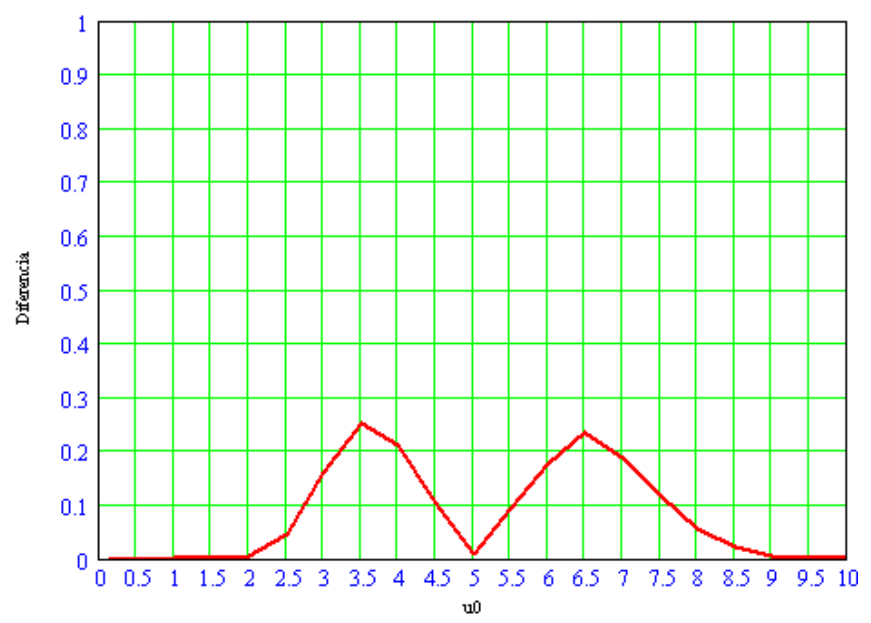

Fig. A.164: $\operatorname{Pot}_{D S-U}-\operatorname{Pot}_{u}$ para $u_{0}=5, n=8$ y $\alpha_{t}=0.05$

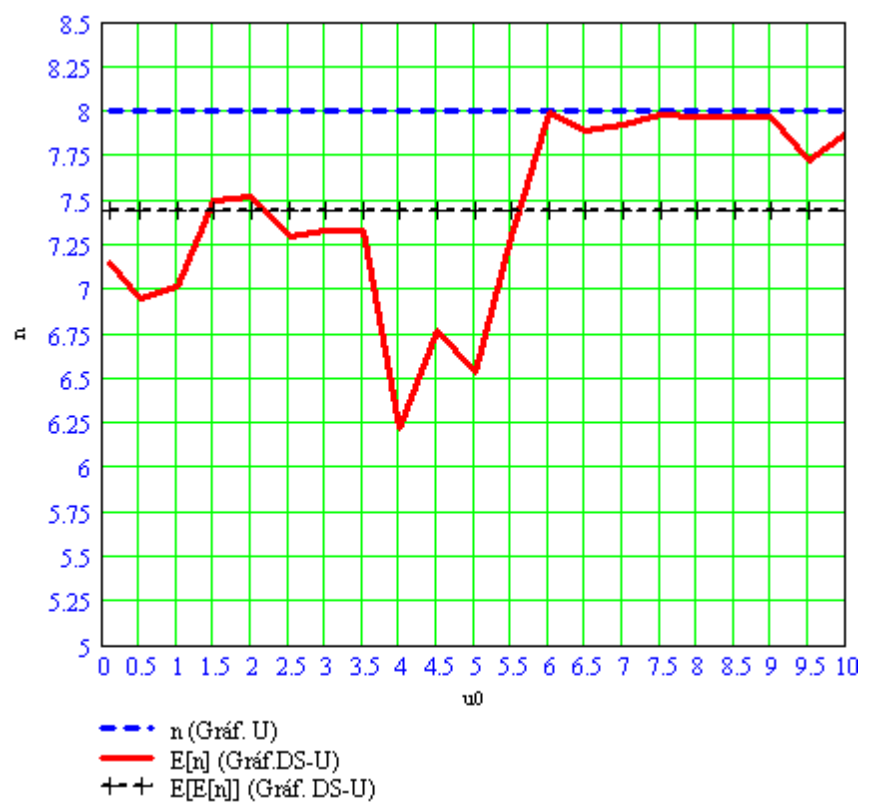

Fig. A.165: Tamaño medio de muestra en el gráfico $D S-U$ para $u_{0}=5, n=8$ y $\alpha_{t}=0.05$ 
APÉNDICE A. COMPARACIÓN DE LA POTENCIA DEL GRÁFICO U FRENTE A LA POTENCIA DEL GRÁFICO DS-U EN UN VALOR U

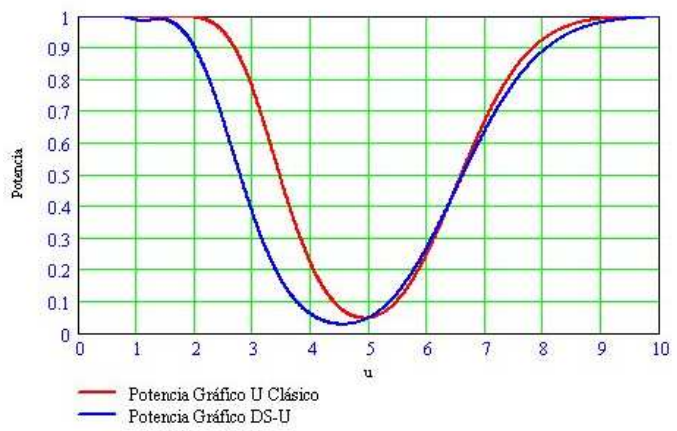

Fig. A.166: Curvas de potencia para $u_{1}=0,1$

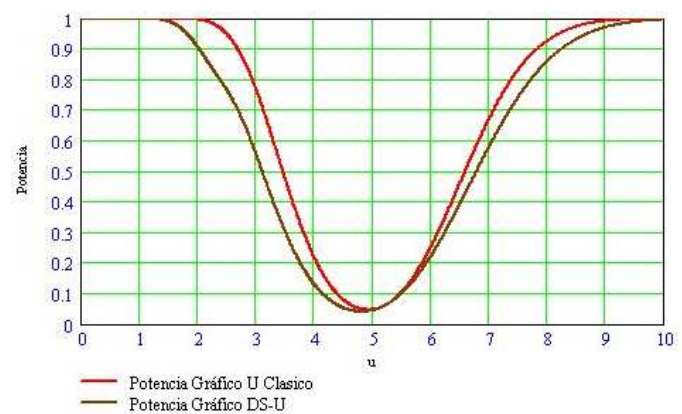

Fig. A.167: Curvas de potencia para $u_{1}=0,5$

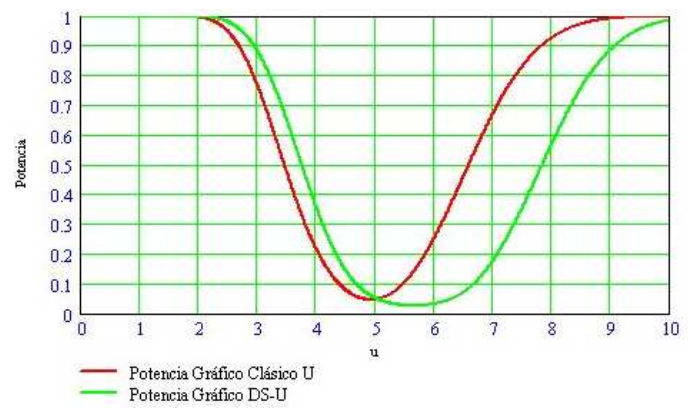

Fig. A.168: Curvas de potencia para $u_{1}=1,0$ 
APÉNDICE A. COMPARACIÓN DE LA POTENCIA DEL GRÁFICO U FRENTE A LA POTENCIA DEL GRÁFICO DS-U EN UN VALOR U

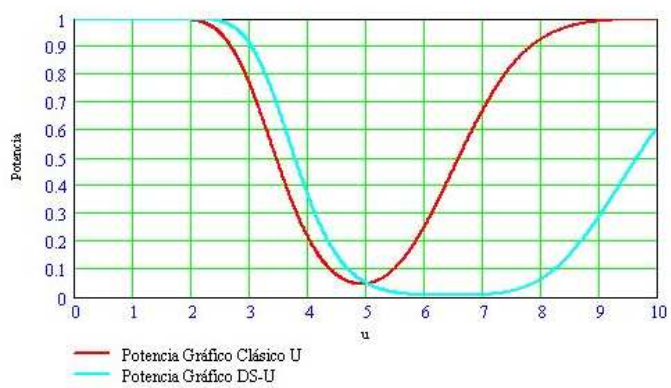

Fig. A.169: Curvas de potencia para $u_{1}=1,5$

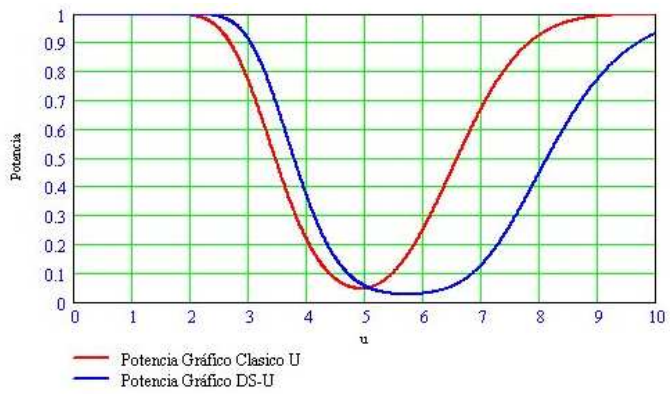

Fig. A.170: Curvas de potencia para $u_{1}=2,0$

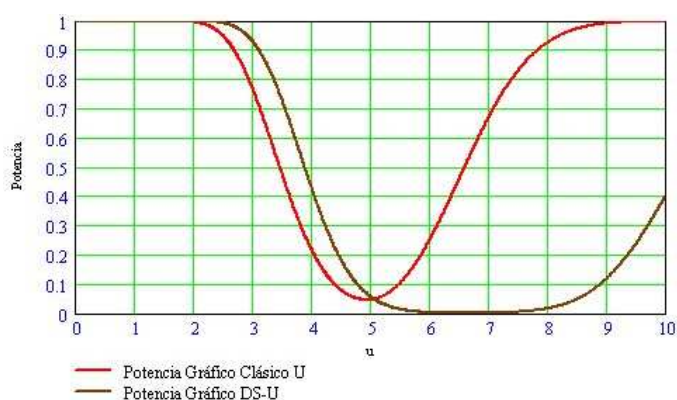

Fig. A.171: Curvas de potencia para $u_{1}=2,5$ 
APÉNDICE A. COMPARACIÓN DE LA POTENCIA DEL GRÁFICO U FRENTE A LA POTENCIA DEL GRÁFICO DS-U EN UN VALOR U

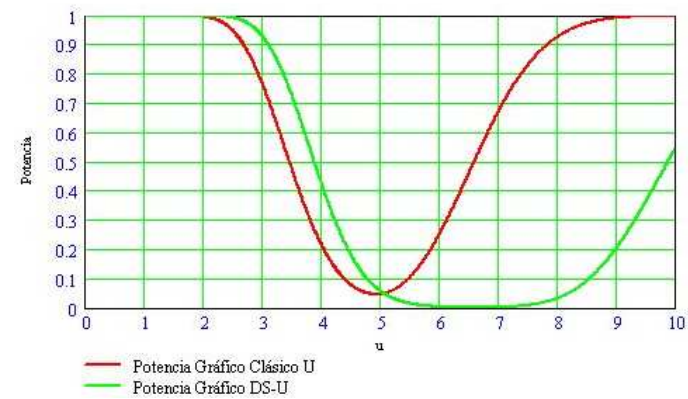

Fig. A.172: Curvas de potencia para $u_{1}=3,0$

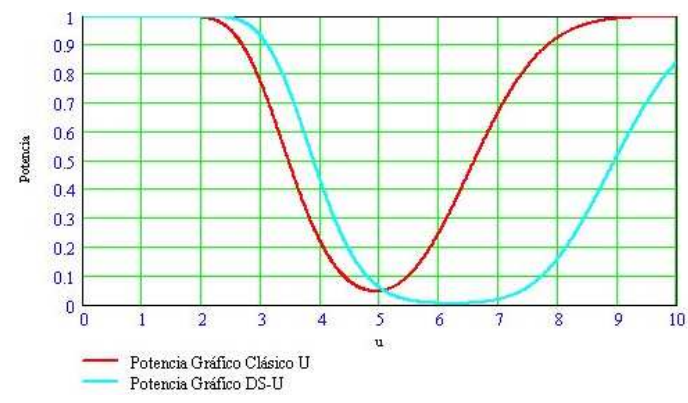

Fig. A.173: Curvas de potencia para $u_{1}=3,5$

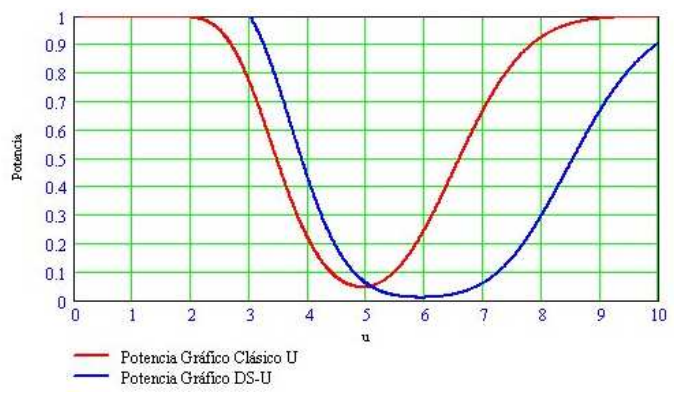

Fig. A.174: Curvas de potencia para $u_{1}=4,0$ 
APÉNDICE A. COMPARACIÓN DE LA POTENCIA DEL GRÁFICO U FRENTE A LA POTENCIA DEL GRÁFICO DS-U EN UN VALOR U

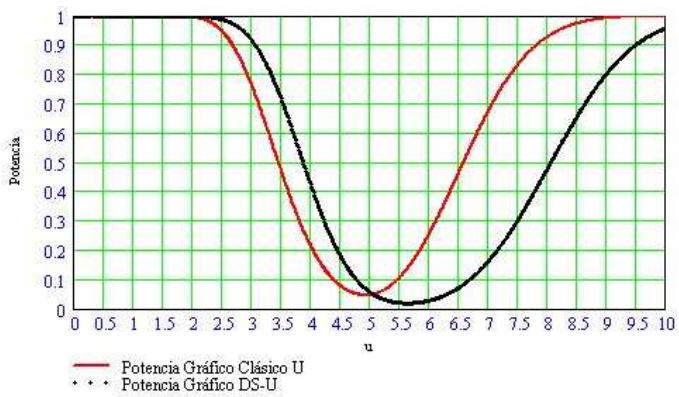

Fig. A.175: Curvas de potencia para $u_{1}=4,5$

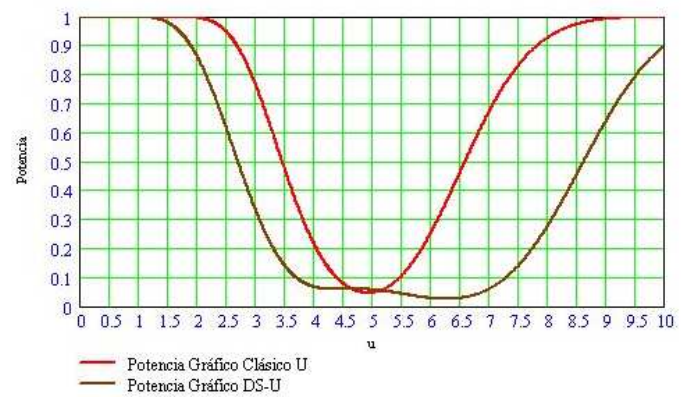

Fig. A.176: Curvas de potencia para $u_{1}=5,0$

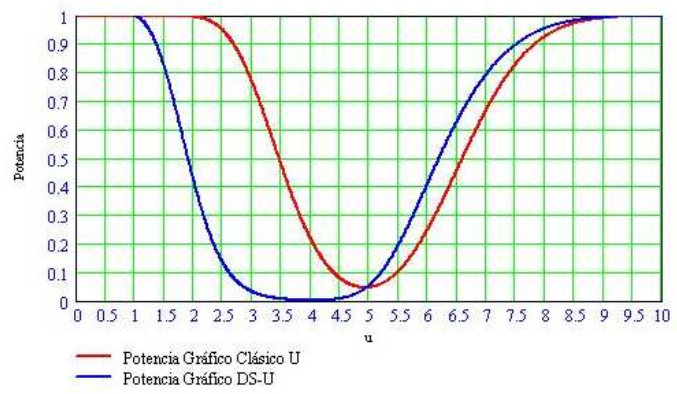

Fig. A.177: Curvas de potencia para $u_{1}=5,5$ 
APÉNDICE A. COMPARACIÓN DE LA POTENCIA DEL GRÁFICO U FRENTE A LA POTENCIA DEL GRÁFICO DS-U EN UN VALOR U

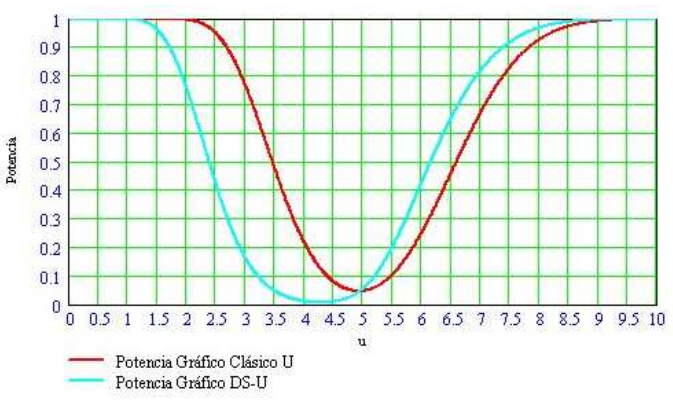

Fig. A.178: Curvas de potencia para $u_{1}=6,0$

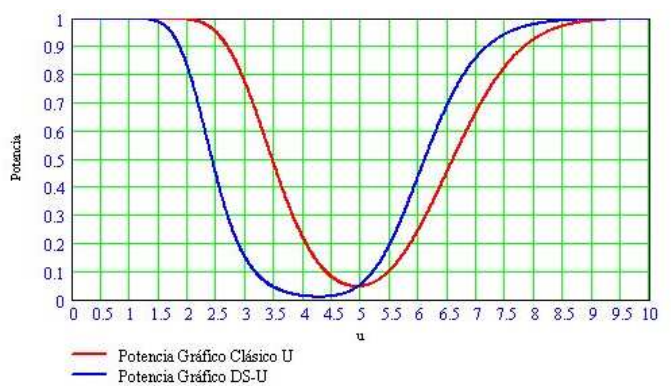

Fig. A.179: Curvas de potencia para $u_{1}=6,5$

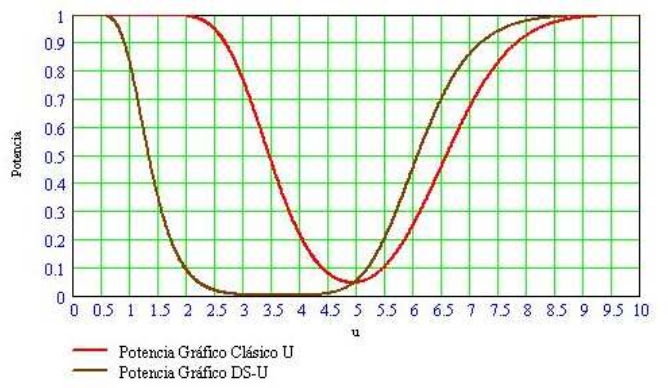

Fig. A.180: Curvas de potencia para $u_{1}=7,0$ 
APÉNDICE A. COMPARACIÓN DE LA POTENCIA DEL GRÁFICO U FRENTE A LA POTENCIA DEL GRÁFICO DS-U EN UN VALOR U

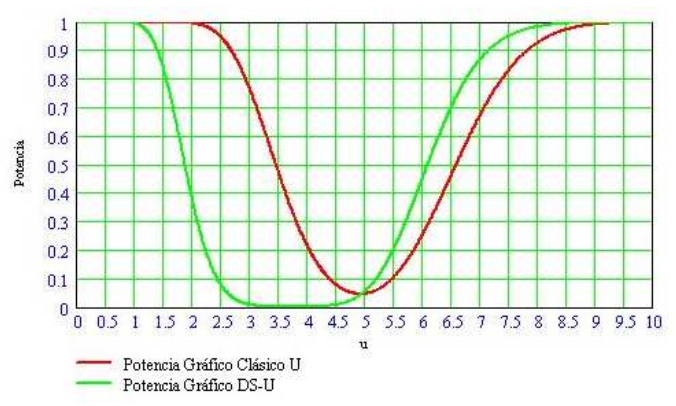

Fig. A.181: Curvas de potencia para $u_{1}=7,5$

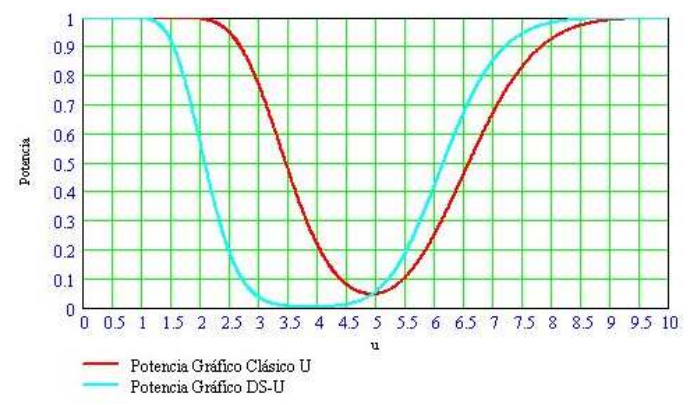

Fig. A.182: Curvas de potencia para $u_{1}=8,0$

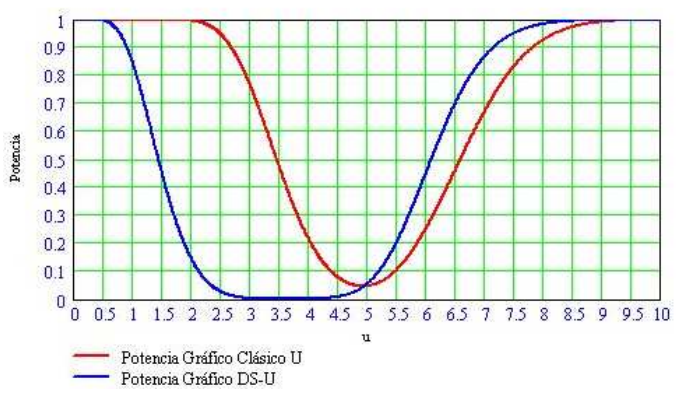

Fig. A.183: Curvas de potencia para $u_{1}=8,5$ 
APÉNDICE A. COMPARACIÓN DE LA POTENCIA DEL GRÁFICO U FRENTE A LA POTENCIA DEL GRÁFICO DS-U EN UN VALOR U

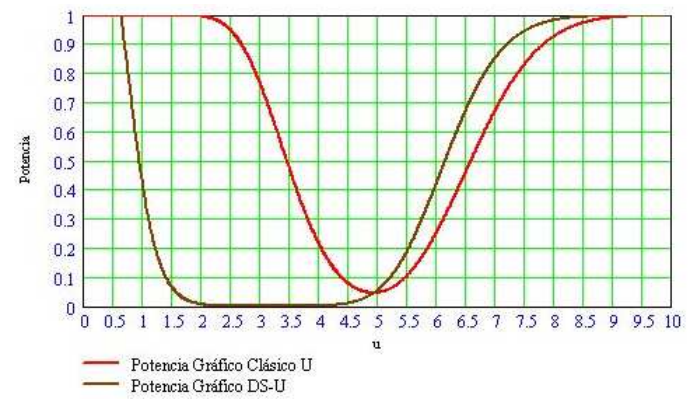

Fig. A.184: Curvas de potencia para $u_{1}=9,0$

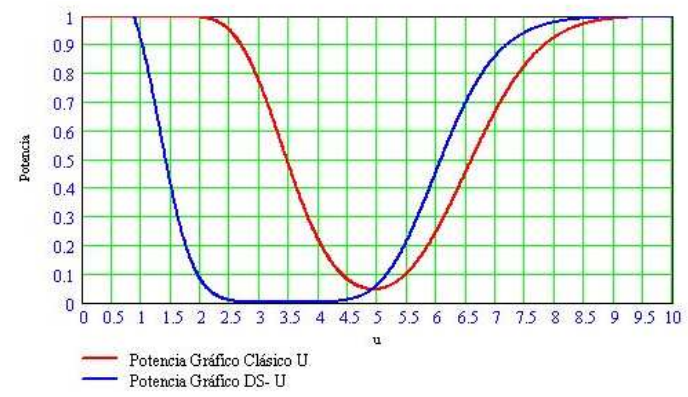

Fig. A.185: Curvas de potencia para $u_{1}=9,5$

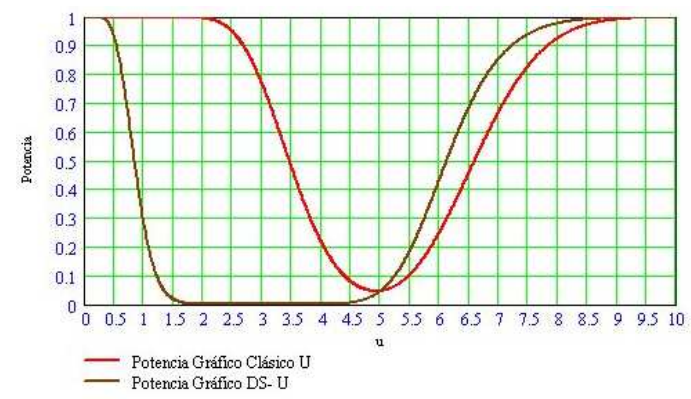

Fig. A.186: Curvas de potencia para $u_{1}=10,0$ 
APÉNDICE A. COMPARACIÓN DE LA POTENCIA DEL GRÁFICO U FRENTE A LA POTENCIA DEL GRÁFICO DS-U EN UN VALOR U

\begin{tabular}{|c||c|c|c|c|c|c||c|c|}
\hline$u_{1}$ & $L C I$ & $L A I$ & $L C I_{1}$ & $L A S$ & $L C S$ & $L C S_{1}$ & $n_{1}$ & $n_{2}$ \\
\hline 0.1 & 11.8 & 16.6 & 21.3 & 39.3 & 41.2 & 43.4 & 6 & 41 \\
0.5 & 16.2 & 18.4 & 24.4 & 40.4 & 52.8 & 54.6 & 6 & 23 \\
1.0 & 3.1 & 24.0 & 21.2 & 39.6 & 51.8 & 40.3 & 6 & 5 \\
1.5 & 16.3 & 23.7 & 24.3 & 47.6 & 57.7 & 55.5 & 5 & 7 \\
2.0 & 16.1 & 23.4 & 24.4 & 39.8 & 49.8 & 48.0 & 5 & 7 \\
2.5 & 14.5 & 23.8 & 23.5 & 48.8 & 54.8 & 60.0 & 5 & 6 \\
3.0 & 14.3 & 23.0 & 23.8 & 38.6 & 58.4 & 59.2 & 5 & 6 \\
3.5 & 14.3 & 23.7 & 23.6 & 39.0 & 53.4 & 52.4 & 5 & 6 \\
4.0 & 14.0 & 21.6 & 20.4 & 39.4 & 45.4 & 42.3 & 5 & 5 \\
4.5 & 17.2 & 24.3 & 22.8 & 45.6 & 48.2 & 48.3 & 6 & 5 \\
\hline 5.0 & 4.4 & 13.2 & 20.9 & 27.5 & 46.7 & 46.9 & 5 & 5 \\
\hline 5.5 & 10.0 & 10.8 & 24.6 & 36.3 & 46.0 & 55.7 & 6 & 11 \\
6.0 & 16.5 & 16.9 & 18.7 & 42.4 & 48.1 & 55.9 & 7 & 11 \\
6.5 & 10.4 & 14.7 & 22.6 & 27.5 & 35.0 & 58.7 & 5 & 10 \\
7.0 & 7.7 & 16.0 & 8.1 & 34.6 & 42.6 & 55.5 & 6 & 10 \\
7.5 & 6.3 & 14.7 & 15.3 & 33.8 & 43.6 & 45.8 & 6 & 8 \\
8.0 & 5.4 & 15.1 & 16.8 & 26.1 & 35.3 & 48.8 & 5 & 8 \\
8.5 & 1.2 & 8.2 & 22.3 & 34.0 & 43.3 & 45.5 & 6 & 8 \\
9.0 & 2.0 & 15.0 & 6.3 & 26.9 & 35.3 & 48.4 & 5 & 8 \\
9.5 & 1.0 & 13.2 & 12.8 & 27.6 & 39.1 & 50.1 & 5 & 9 \\
10.0 & 0.5 & 8.5 & 8.9 & 34.3 & 42.7 & 56.4 & 6 & 10 \\
\hline
\end{tabular}

Tabla A.15: Parámetros obtenidos para la comparación de la potencia para el nuevo gráfico $D S-U: u_{0}=5, \alpha_{t}=0.05$, $n=8$. 
APÉNDICE A. COMPARACIÓN DE LA POTENCIA DEL GRÁFICO U FRENTE A LA POTENCIA DEL GRÁFICO DS-U EN UN VALOR U

\begin{tabular}{|c||c|c||c|c|}
\hline$u_{1}$ & Pot $_{U}$ & Pot $_{D S-U}$ & Pot $_{D S-U}-$ Pot $_{U}$ & $\alpha_{\text {real }}$ \\
\hline 0.1 & 1 & 1 & 0 & 0.050126 \\
0.5 & 1 & 1 & $-5.667984 \cdot 10^{-10}$ & 0.045242 \\
1.0 & 1 & 1 & $7.914983 \cdot 10^{-9}$ & 0.050864 \\
1.5 & 0.999944 & 0.999999 & $5.437253 \cdot 10^{-5}$ & 0.049510 \\
2.0 & 0.995895 & 0.999745 & $3.849797 \cdot 10^{-3}$ & 0.054636 \\
2.5 & 0.947519 & 0.992231 & 0.044712 & 0.056138 \\
3.0 & 0.767742 & 0.928476 & 0.160734 & 0.056790 \\
3.5 & 0.474881 & 0.727137 & 0.252256 & 0.056826 \\
4.0 & 0.216646 & 0.427812 & 0.211167 & 0.057962 \\
4.5 & 0.078269 & 0.180277 & 0.102008 & 0.056755 \\
\hline 5.0 & 0.047396 & 0.056875 & $9.478921 \cdot 10^{-3}$ & 0.056875 \\
\hline 5.5 & 0.106533 & 0.198396 & 0.091863 & 0.056876 \\
6.0 & 0.254211 & 0.430334 & 0.176124 & 0.056838 \\
6.5 & 0.463311 & 0.698836 & 0.235525 & 0.056704 \\
7.0 & 0.673712 & 0.862771 & 0.189059 & 0.056147 \\
7.5 & 0.833356 & 0.951494 & 0.118138 & 0.056703 \\
8.0 & 0.928208 & 0.982497 & 0.054289 & 0.056720 \\
8.5 & 0.973672 & 0.994931 & 0.021259 & 0.056871 \\
9.0 & 0.991689 & 0.998347 & $6.658319 \cdot 10^{-3}$ & 0.056713 \\
9.5 & 0.997716 & 0.999112 & $1.396746 \cdot 10^{-3}$ & 0.065138 \\
10.0 & 0.999447 & 0.999812 & $3.648061 \cdot 10^{-4}$ & 0.048239 \\
\hline
\end{tabular}

Tabla A.16: Resultados para $u_{0}=5, \alpha_{\text {teórico }}=0.05$ y $n=8$ 
APÉNDICE A. COMPARACIÓN DE LA POTENCIA DEL GRÁFICO U FRENTE A LA POTENCIA DEL GRÁFICO DS-U EN UN VALOR U

A.9. Comparación de los gráficos de control $u$ y $D S-U$ con $n=8, u_{0}=5$ y $\alpha_{t}=0.0027$ 
APÉNDICE A. COMPARACIÓN DE LA POTENCIA DEL GRÁFICO U FRENTE A LA POTENCIA DEL GRÁFICO DS-U EN UN VALOR U

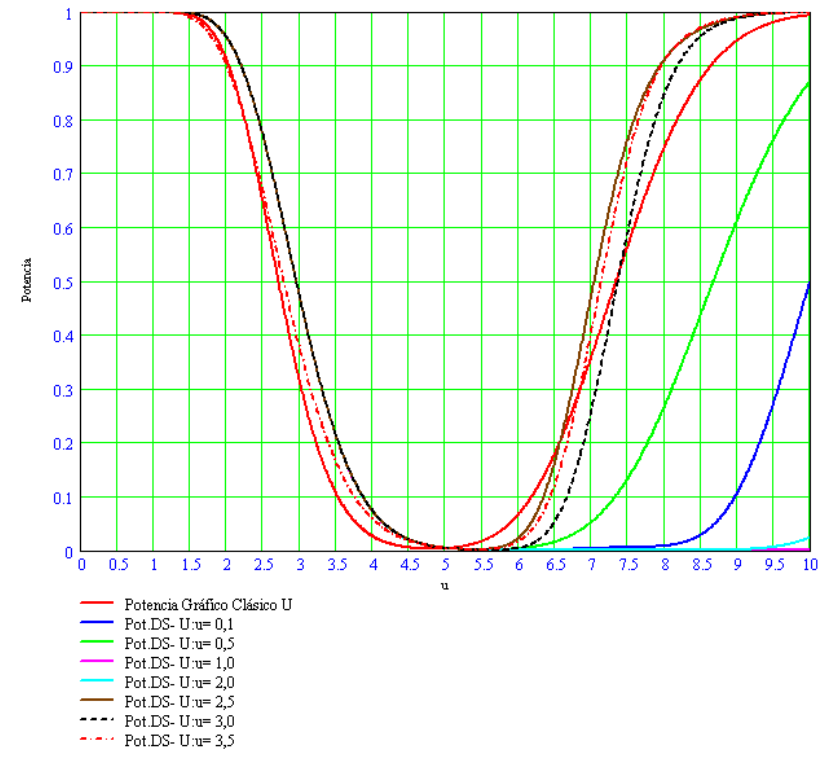

(a) $u_{1} \in[0.1,5.0]$

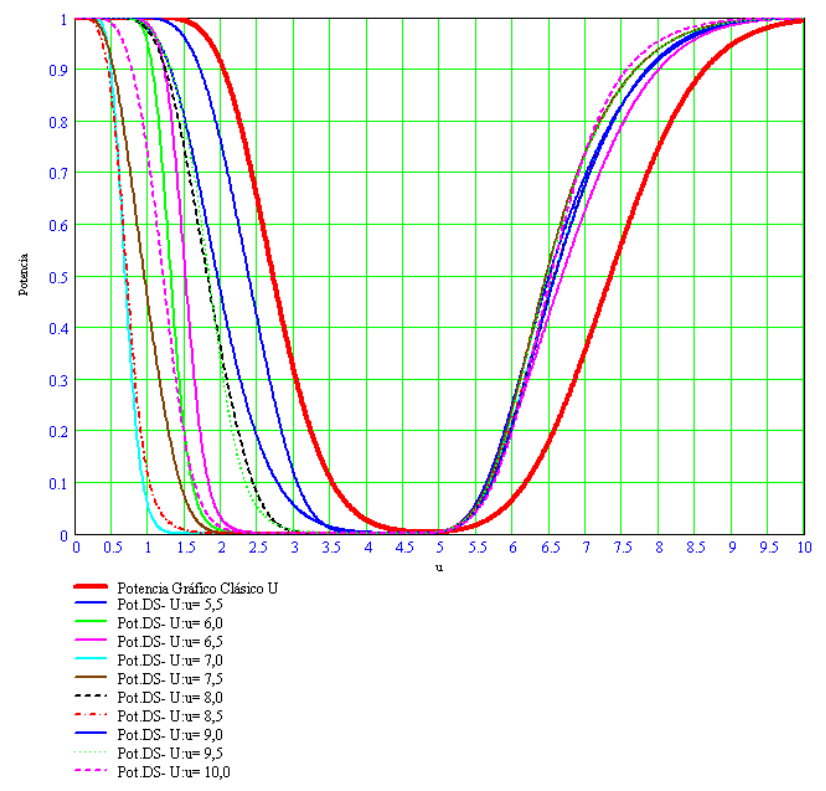

(b) $u_{1} \in[5.5,10.0]$

Fig. A.187: Curvas de potencia en distintos valores de $u_{1}$ 
APÉNDICE A. COMPARACIÓN DE LA POTENCIA DEL GRÁFICO U FRENTE A LA POTENCIA DEL GRÁFICO DS-U EN UN VALOR U

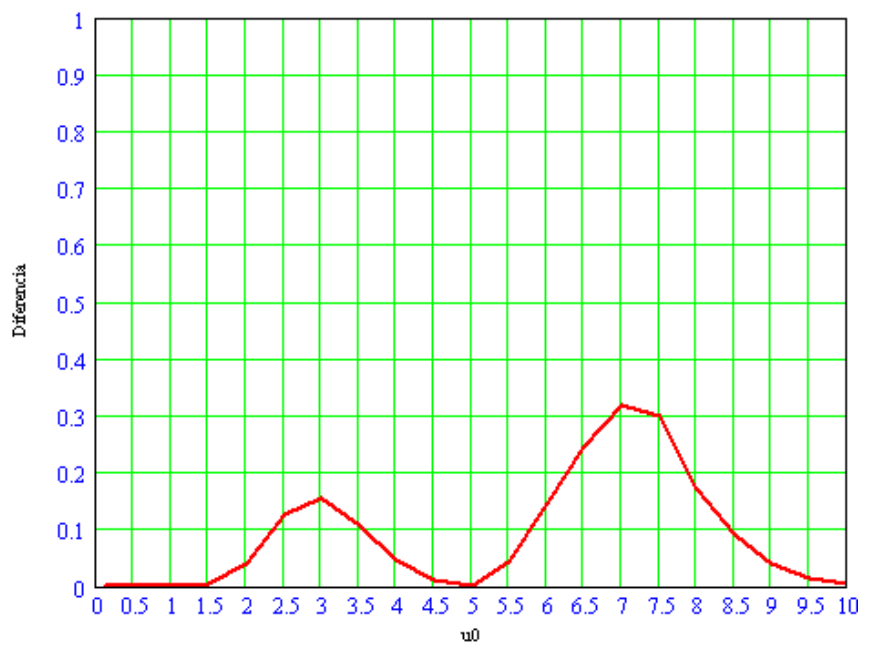

Fig. A.188: $\operatorname{Pot}_{D S-U}-$ Pot $_{u}$ para $n=8, u_{0}=5$ y $\alpha_{t}=0.0027$

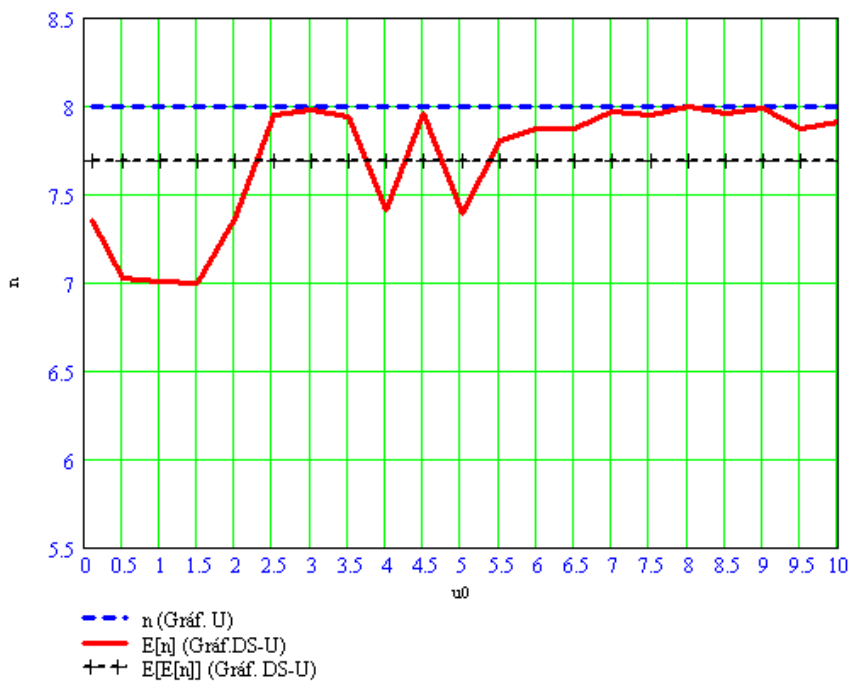

Fig. A.189: Tamaño medio de muestra en el gráfico $D S-U$ para $n=8, u_{0}=5$ y $\alpha_{t}=0.0027$ 
APÉNDICE A. COMPARACIÓN DE LA POTENCIA DEL GRÁFICO U FRENTE A LA POTENCIA DEL GRÁFICO DS-U EN UN VALOR U

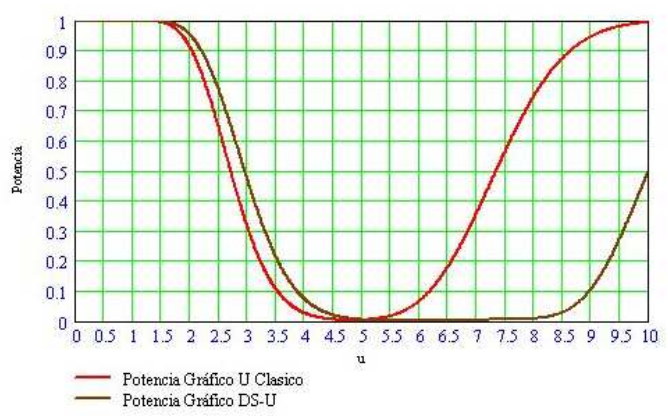

Fig. A.190: Curvas de potencia para $u_{1}=0,5$

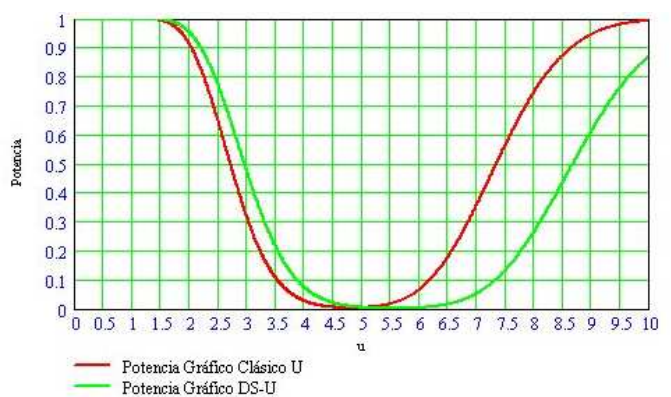

Fig. A.191: Curvas de potencia para $u_{1}=1,0$

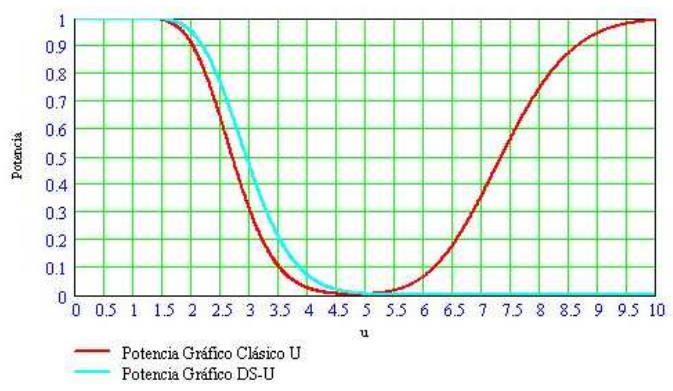

Fig. A.192: Curvas de potencia para $u_{1}=1,5$ 
APÉNDICE A. COMPARACIÓN DE LA POTENCIA DEL GRÁFICO U FRENTE A LA POTENCIA DEL GRÁFICO DS-U EN UN VALOR U

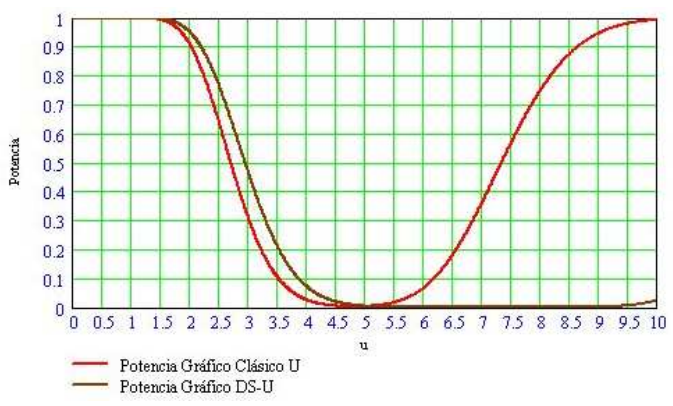

Fig. A.193: Curvas de potencia para $u_{1}=2,5$

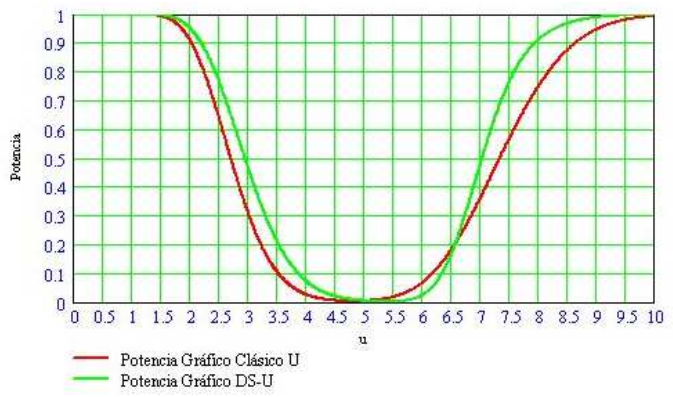

Fig. A.194: Curvas de potencia para $u_{1}=3,0$

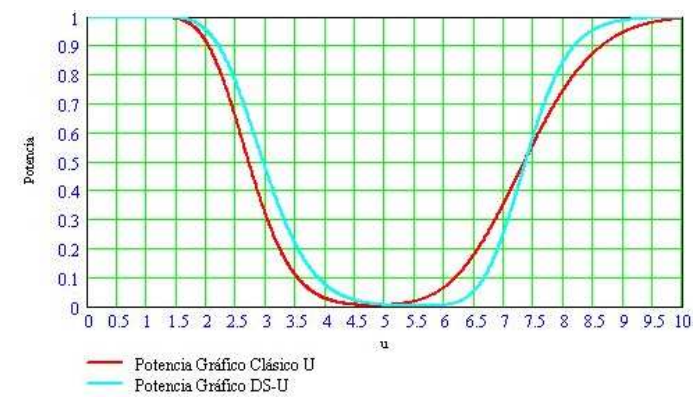

Fig. A.195: Curvas de potencia para $u_{1}=3,5$ 
APÉNDICE A. COMPARACIÓN DE LA POTENCIA DEL GRÁFICO U FRENTE A LA POTENCIA DEL GRÁFICO DS-U EN UN VALOR U

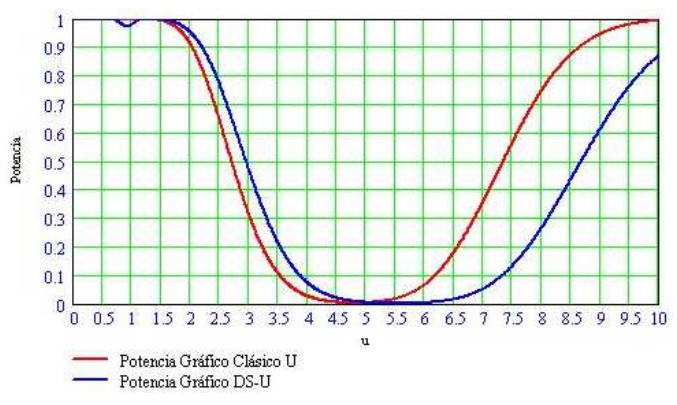

Fig. A.196: Curvas de potencia para $u_{1}=4,0$

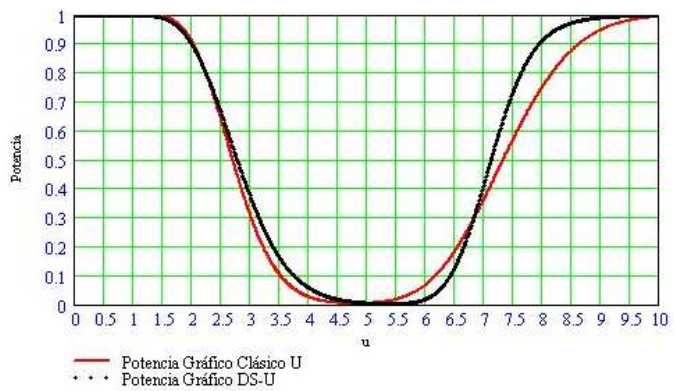

Fig. A.197: Curvas de potencia para $u_{1}=4,5$

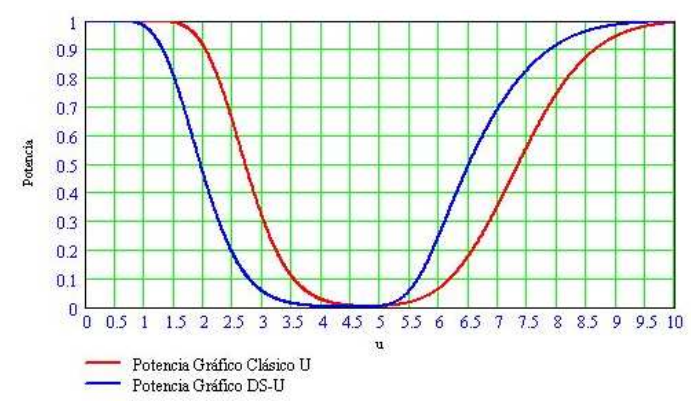

Fig. A.198: Curvas de potencia para $u_{1}=5,5$ 
APÉNDICE A. COMPARACIÓN DE LA POTENCIA DEL GRÁFICO U FRENTE A LA POTENCIA DEL GRÁFICO DS-U EN UN VALOR U

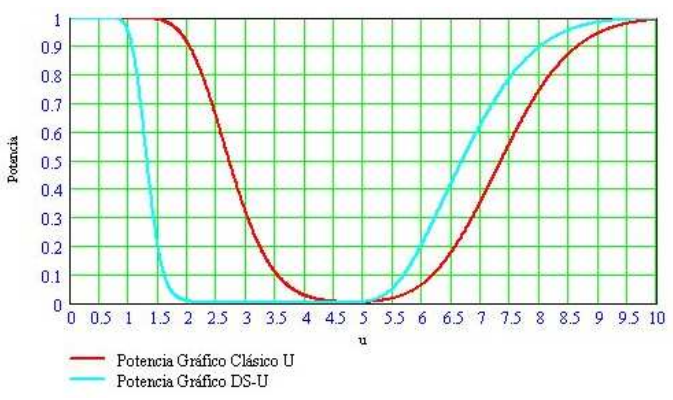

Fig. A.199: Curvas de potencia para $u_{1}=6,0$

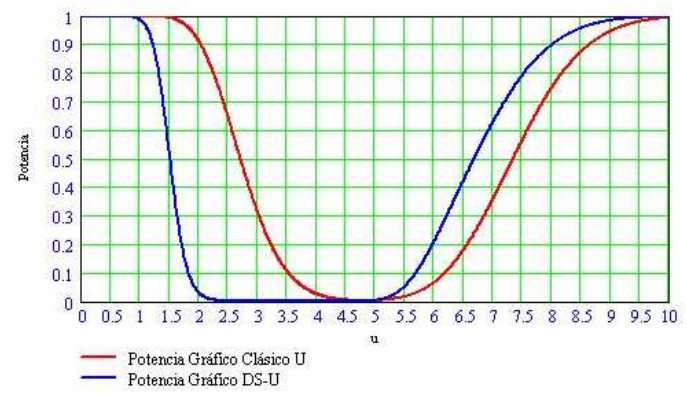

Fig. A.200: Curvas de potencia para $u_{1}=6,5$

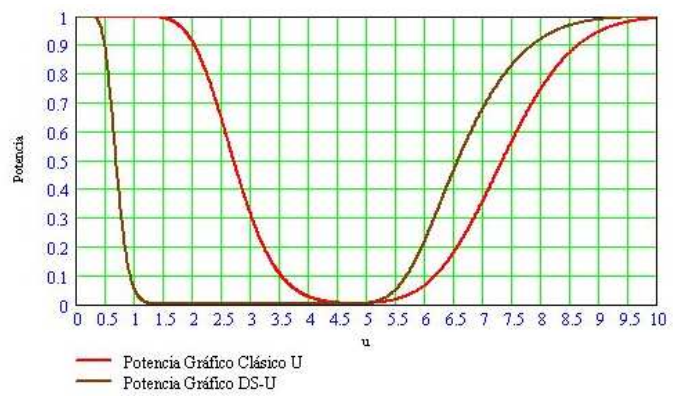

Fig. A.201: Curvas de potencia para $u_{1}=7,0$ 
APÉNDICE A. COMPARACIÓN DE LA POTENCIA DEL GRÁFICO U FRENTE A LA POTENCIA DEL GRÁFICO DS-U EN UN VALOR U

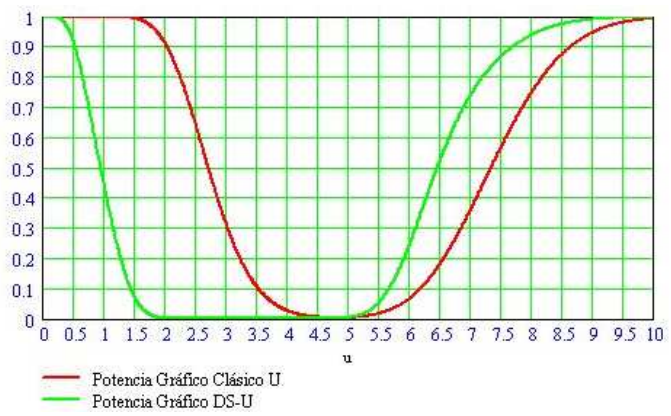

Fig. A.202: Curvas de potencia para $u_{1}=7,5$

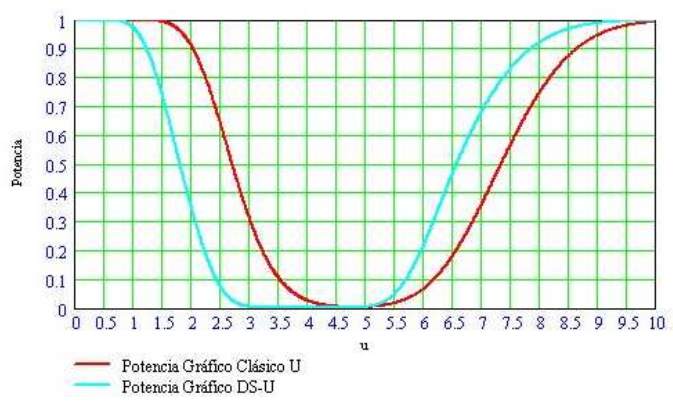

Fig. A.203: Curvas de potencia para $u_{1}=8,0$

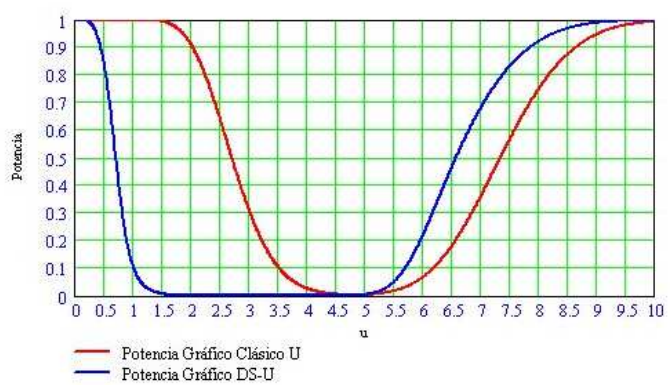

Fig. A.204: Curvas de potencia para $u_{1}=8,5$ 
APÉNDICE A. COMPARACIÓN DE LA POTENCIA DEL GRÁFICO U FRENTE A LA POTENCIA DEL GRÁFICO DS-U EN UN VALOR U

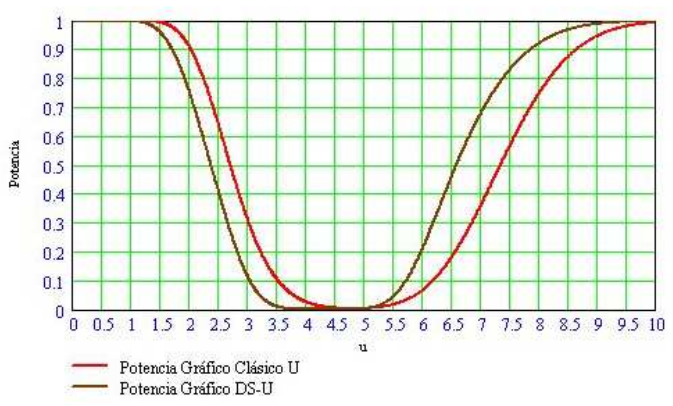

Fig. A.205: Curvas de potencia para $u_{1}=9,0$

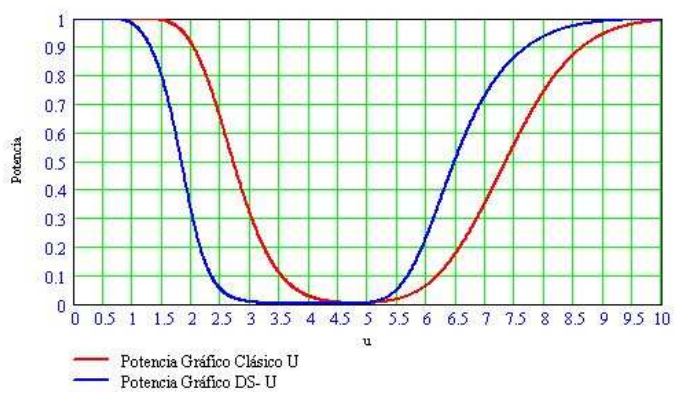

Fig. A.206: Curvas de potencia para $u_{1}=9,5$

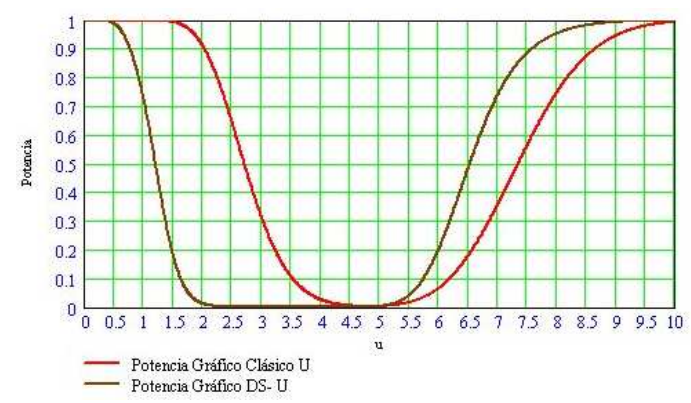

Fig. A.207: Curvas de potencia para $u_{1}=10,0$ 
APÉNDICE A. COMPARACIÓN DE LA POTENCIA DEL GRÁFICO U FRENTE A LA POTENCIA DEL GRÁFICO DS-U EN UN VALOR U

\begin{tabular}{|c||c|c|c|c|c|c||c|c|}
\hline$u_{1}$ & $L C I$ & $L A I$ & $L C I_{1}$ & $L A S$ & $L C S$ & $L C S_{1}$ & $n_{1}$ & $n_{2}$ \\
\hline 0.1 & 11.7 & 20.8 & 45.7 & 73.9 & 136.8 & 106.2 & 7 & 82 \\
0.5 & 19.1 & 20.7 & 79.5 & 65.7 & 113.8 & 113.4 & 7 & 12 \\
1.0 & 20.1 & 20.3 & 88.4 & 60.3 & 66.9 & 100.2 & 7 & 23 \\
1.5 & 20.1 & 20.6 & 78.8 & 115.0 & 122.0 & 129.2 & 7 & 85 \\
2.0 & 2.3 & 20.4 & 24.7 & 74.9 & 111.9 & 94.0 & 7 & 84 \\
2.5 & 20.5 & 20.8 & 24.9 & 42.7 & 94.4 & 109.8 & 7 & 9 \\
3.0 & 20.4 & 20.7 & 84.0 & 45.4 & 65.9 & 154.5 & 7 & 23 \\
3.5 & 20.2 & 20.8 & 79.5 & 45.5 & 97.5 & 157.1 & 7 & 22 \\
4.0 & 10.2 & 20.6 & 72.9 & 60.7 & 157.0 & 86.9 & 7 & 95 \\
4.5 & 16.1 & 16.6 & 83.5 & 37.9 & 52.0 & 153.3 & 6 & 22 \\
\hline 5.0 & 4.3 & 20.4 & 20.0 & 60.3 & 159.2 & 137.2 & 7 & 89 \\
\hline 5.5 & 11.7 & 11.9 & 99.4 & 38.3 & 50.6 & 158.2 & 6 & 28 \\
6.0 & 5.6 & 13.1 & 38.0 & 46.7 & 56.5 & 158.6 & 7 & 29 \\
6.5 & 6.8 & 13.6 & 44.8 & 47.0 & 56.5 & 158.7 & 7 & 29 \\
7.0 & 0.5 & 14.3 & 15.7 & 45.6 & 56.1 & 129.8 & 7 & 23 \\
7.5 & 1.9 & 5.4 & 34.4 & 37.5 & 49.5 & 128.1 & 6 & 22 \\
8.0 & 1.3 & 12.7 & 63.6 & 45.6 & 54.8 & 136.1 & 7 & 24 \\
8.5 & 3.8 & 5.3 & 17.5 & 45.3 & 54.3 & 130.1 & 7 & 23 \\
9.0 & 5.1 & 16.9 & 73.6 & 46.0 & 150.4 & 130.0 & 7 & 23 \\
9.5 & 8.5 & 11.8 & 42.9 & 37.4 & 133.9 & 122.3 & 6 & 21 \\
10.0 & 4.3 & 7.2 & 23.1 & 36.2 & 49.1 & 97.2 & 6 & 16 \\
\hline
\end{tabular}

Tabla A.17: Parámetros obtenidos en la comparación de la potencia para el nuevo gráfico $D S-U: u_{0}=5, \alpha_{t}=0.0027$, $n=8$. 
APÉNDICE A. COMPARACIÓN DE LA POTENCIA DEL GRÁFICO U FRENTE A LA POTENCIA DEL GRÁFICO DS-U EN UN VALOR U

\begin{tabular}{|c||c|c||c|c|}
\hline$u_{1}$ & Pot $_{U}$ & Pot $_{D S-U}$ & Pot $_{D S-U}-$ Pot $_{U}$ & $\alpha_{\text {real }}$ \\
\hline 0.1 & 1 & 1 & 0 & $4.296615 \cdot 10^{-3}$ \\
0.5 & 1 & 1 & $1.594804 \cdot 10^{-10}$ & $4.283109 \cdot 10^{-3}$ \\
1.0 & 0.999967 & 0.999986 & $1.891199 \cdot 10^{-5}$ & $4.336190 \cdot 10^{-3}$ \\
1.5 & 0.993935 & 0.997212 & $3.277319 \cdot 10^{-3}$ & $4.296609 \cdot 10^{-3}$ \\
2.0 & 0.910773 & 0.952092 & 0.041318 & $4.296612 \cdot 10^{-3}$ \\
2.5 & 0.643698 & 0.769435 & 0.125737 & $4.343793 \cdot 10^{-3}$ \\
3.0 & 0.313928 & 0.470976 & 0.157048 & $4.352933 \cdot 10^{-3}$ \\
3.5 & 0.105986 & 0.212798 & 0.106811 & $4.346597 \cdot 10^{-3}$ \\
4.0 & 0.026008 & 0.072741 & 0.046733 & $4.339577 \cdot 10^{-3}$ \\
4.5 & $5.150110 \cdot 10^{-3}$ & 0.017505 & 0.012355 & $4.356869 \cdot 10^{-3}$ \\
\hline 5.0 & $3.631613 \cdot 10^{-3}$ & $4.339577 \cdot 10^{-3}$ & $7.079646 \cdot 10^{-4}$ & $4.339577 \cdot 10^{-3}$ \\
\hline 5.5 & 0.017787 & 0.061018 & 0.043231 & $4.323354 \cdot 10^{-3}$ \\
6.0 & 0.068373 & 0.212920 & 0.144547 & $4.331676 \cdot 10^{-3}$ \\
6.5 & 0.182409 & 0.426781 & 0.244371 & $4.331677 \cdot 10^{-3}$ \\
7.0 & 0.361748 & 0.682225 & 0.320477 & $4.185062 \cdot 10^{-3}$ \\
7.5 & 0.568601 & 0.868864 & 0.300263 & $4.194640 \cdot 10^{-3}$ \\
8.0 & 0.750648 & 0.923307 & 0.172659 & $3.896531 \cdot 10^{-3}$ \\
8.5 & 0.876936 & 0.969368 & 0.092431 & $4.230131 \cdot 10^{-3}$ \\
9.0 & 0.947886 & 0.989240 & 0.041353 & $3.266117 \cdot 10^{-3}$ \\
9.5 & 0.980911 & 0.996858 & 0.015947 & $4.148510 \cdot 10^{-3}$ \\
10.0 & 0.993895 & 0.999424 & $5.528115 \cdot 10^{-3}$ & $3.868992 \cdot 10^{-3}$ \\
\hline
\end{tabular}

Tabla A.18: Resultados para $u_{0}=5, \alpha_{\text {teorico }}=0.0027$ y $n=8$ 
APÉNDICE A. COMPARACIÓN DE LA POTENCIA DEL GRÁFICO U FRENTE A LA POTENCIA DEL GRÁFICO DS-U EN UN VALOR U

A.10. Comparación de los gráficos de control $u$ y $\boldsymbol{D} \boldsymbol{S}-\boldsymbol{U} \operatorname{con} n=40, u_{0}=5$ y $\alpha_{t}=0.01$ 
APÉNDICE A. COMPARACIÓN DE LA POTENCIA DEL GRÁFICO U FRENTE A LA POTENCIA DEL GRÁFICO DS-U EN UN VALOR U

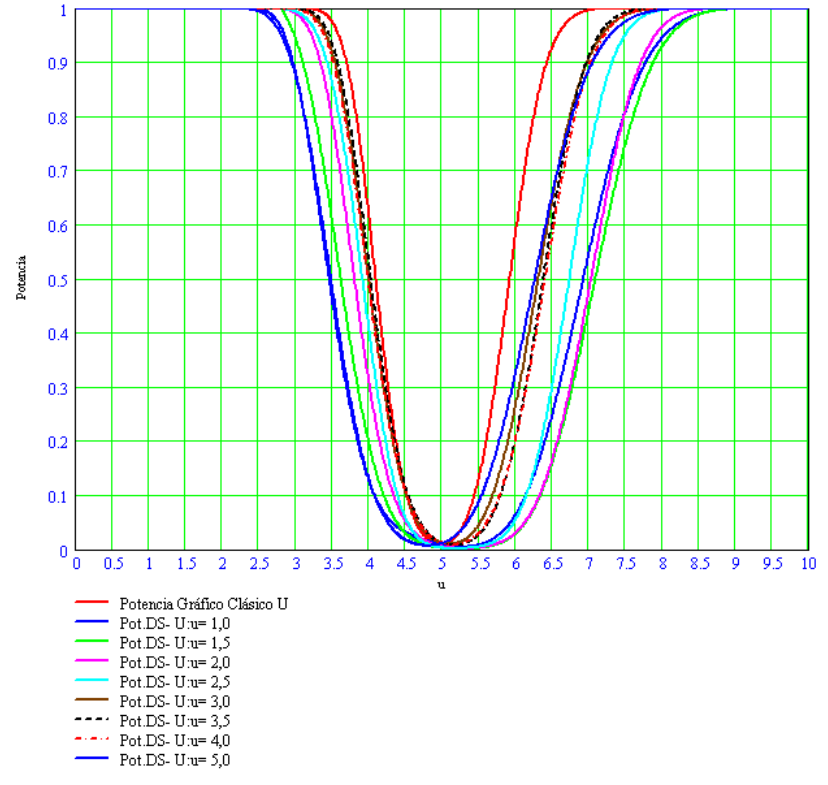

(a) $u_{1} \in[0.1,5.0]$

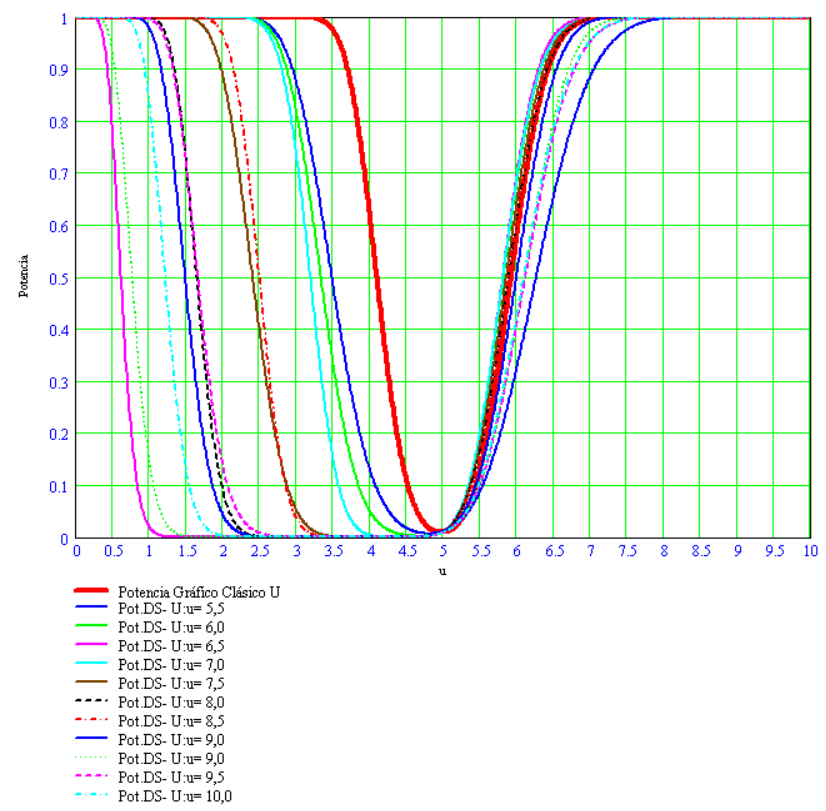

(b) $u_{1} \in[5.5,10.0]$

Fig. A.208: Curvas de potencia en distintos valores de $u_{1}$ 
APÉNDICE A. COMPARACIÓN DE LA POTENCIA DEL GRÁFICO U FRENTE A LA POTENCIA DEL GRÁFICO DS-U EN UN VALOR U

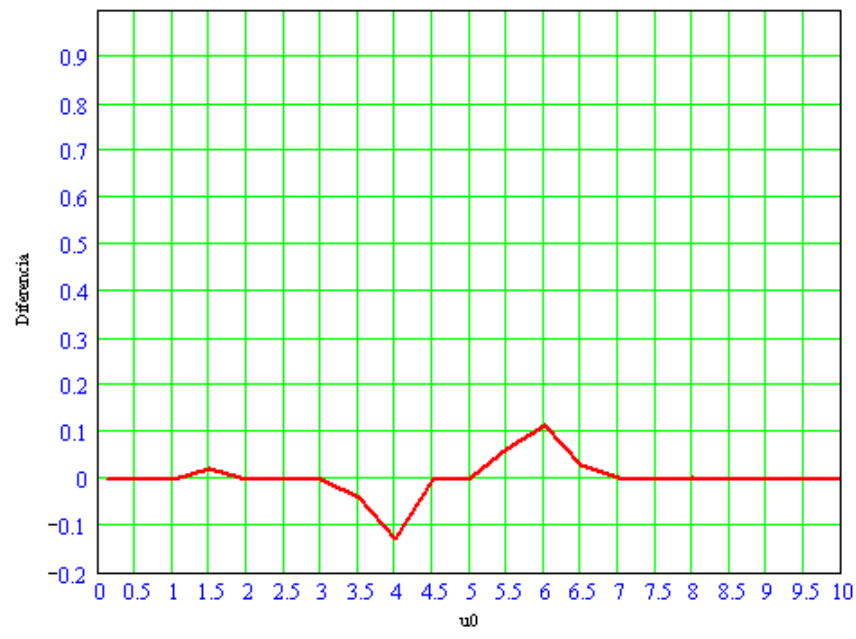

Fig. A.209: $\operatorname{Pot}_{D S-U}-$ Pot $_{u}$ para $n=40, u_{0}=5$ y $\alpha_{t}=0.01$

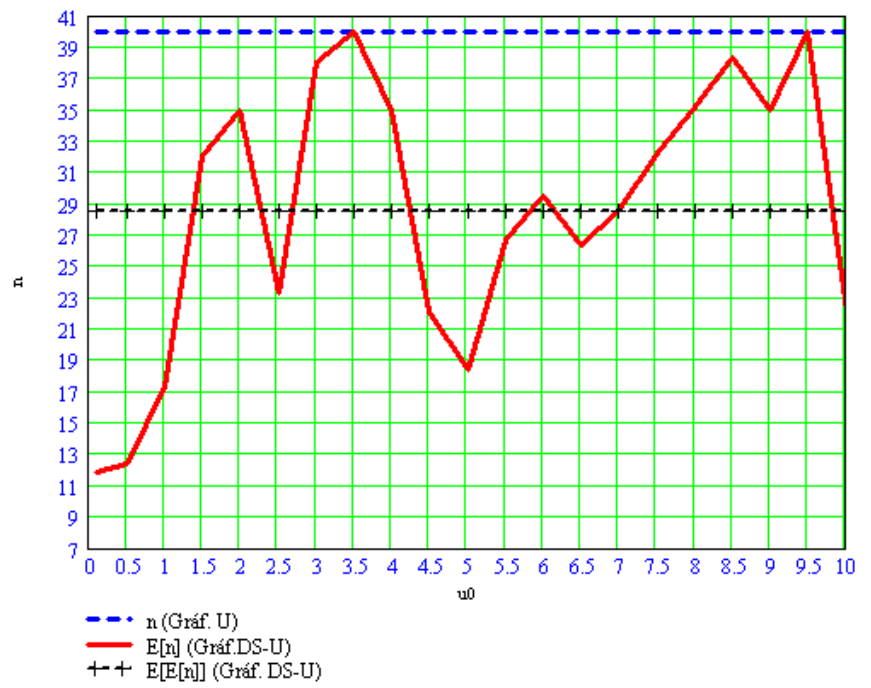

Fig. A.210: Tamaño medio de muestra en el gráfico $D S-U$ para $n=40, u_{0}=5$ y $\alpha_{t}=0.01$ 
APÉNDICE A. COMPARACIÓN DE LA POTENCIA DEL GRÁFICO U FRENTE A LA POTENCIA DEL GRÁFICO DS-U EN UN VALOR U

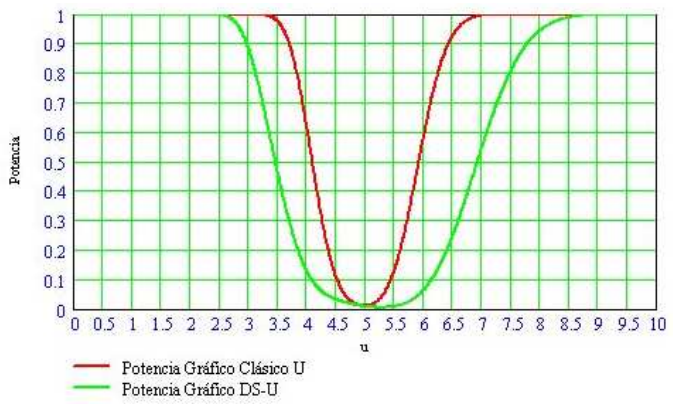

Fig. A.211: Curvas de potencia para $u_{1}=1,0$

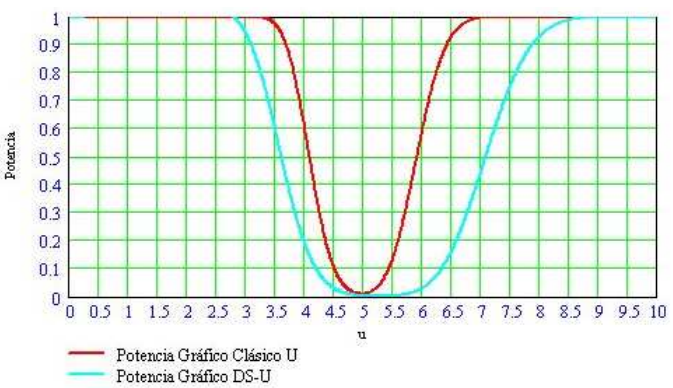

Fig. A.212: Curvas de potencia para $u_{1}=1,5$

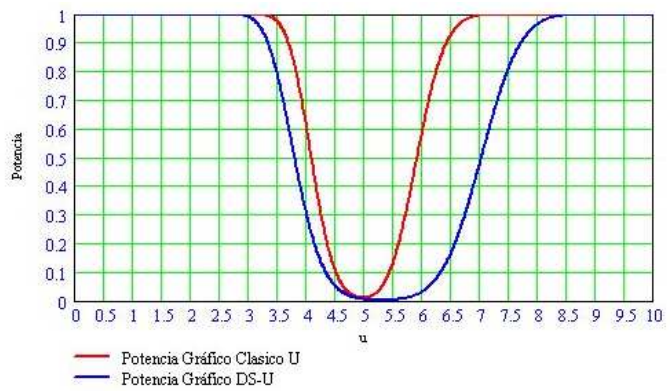

Fig. A.213: Curvas de potencia para $u_{1}=2,0$ 
APÉNDICE A. COMPARACIÓN DE LA POTENCIA DEL GRÁFICO U FRENTE A LA POTENCIA DEL GRÁFICO DS-U EN UN VALOR U

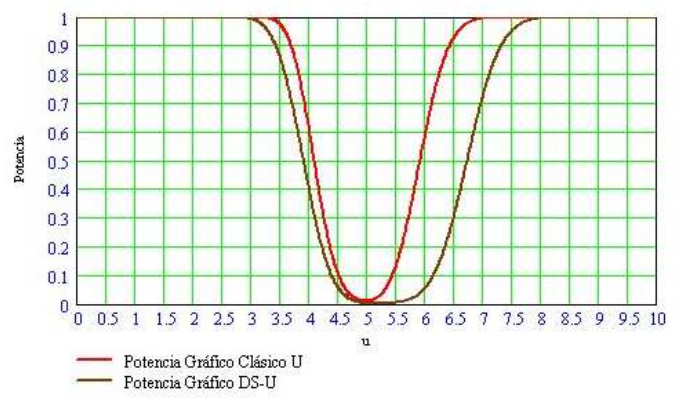

Fig. A.214: Curvas de potencia para $u_{1}=2,5$

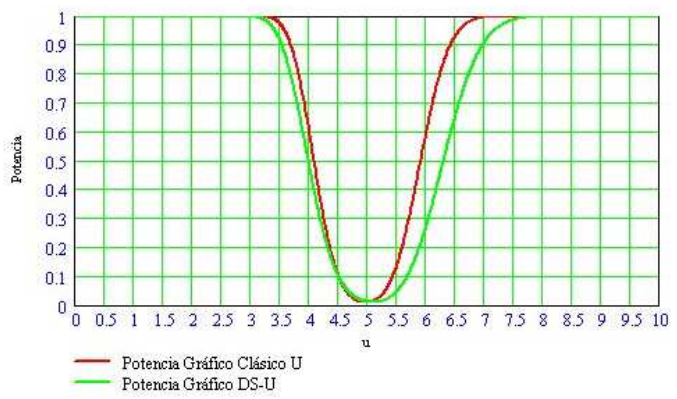

Fig. A.215: Curvas de potencia para $u_{1}=3,0$

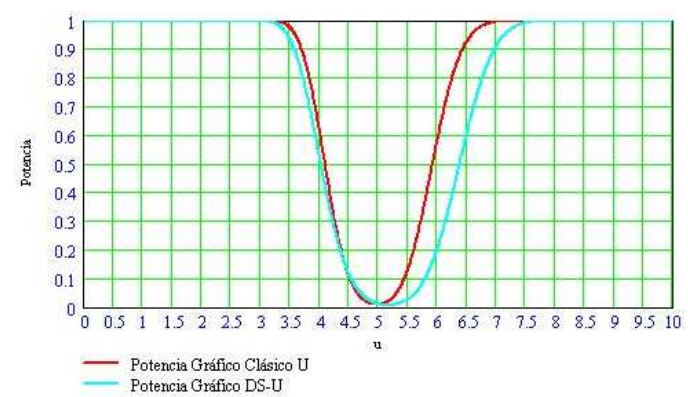

Fig. A.216: Curvas de potencia para $u_{1}=3,5$ 
APÉNDICE A. COMPARACIÓN DE LA POTENCIA DEL GRÁFICO U FRENTE A LA POTENCIA DEL GRÁFICO DS-U EN UN VALOR U

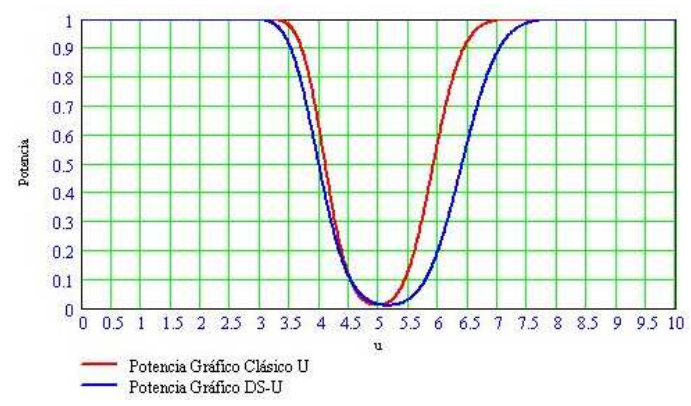

Fig. A.217: Curvas de potencia para $u_{1}=4,0$

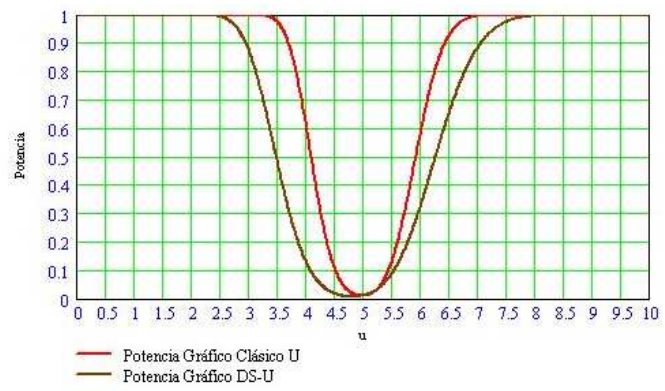

Fig. A.218: Curvas de potencia para $u_{1}=5,0$

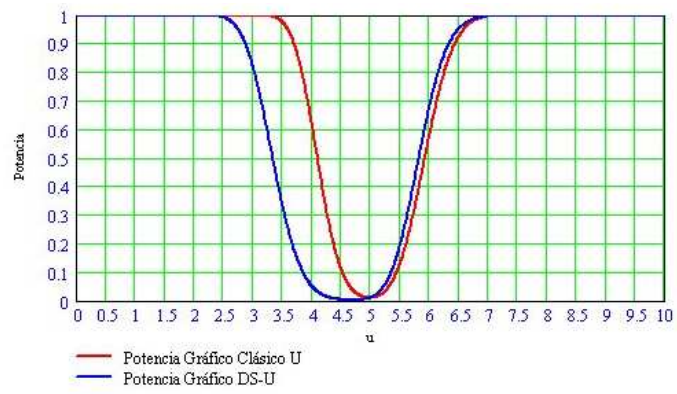

Fig. A.219: Curvas de potencia para $u_{1}=5,5$ 
APÉNDICE A. COMPARACIÓN DE LA POTENCIA DEL GRÁFICO U FRENTE A LA POTENCIA DEL GRÁFICO DS-U EN UN VALOR U

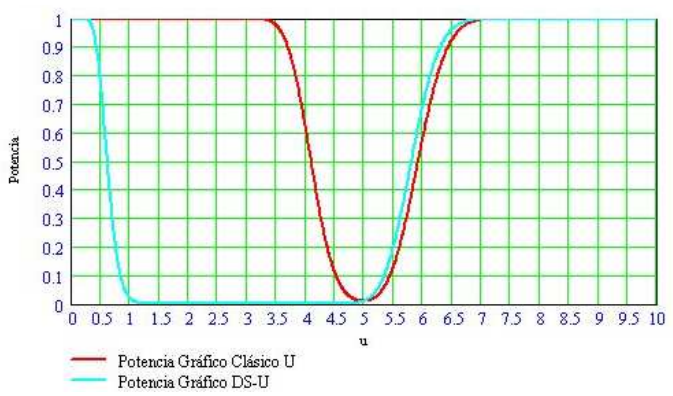

Fig. A.220: Curvas de potencia para $u_{1}=6,0$

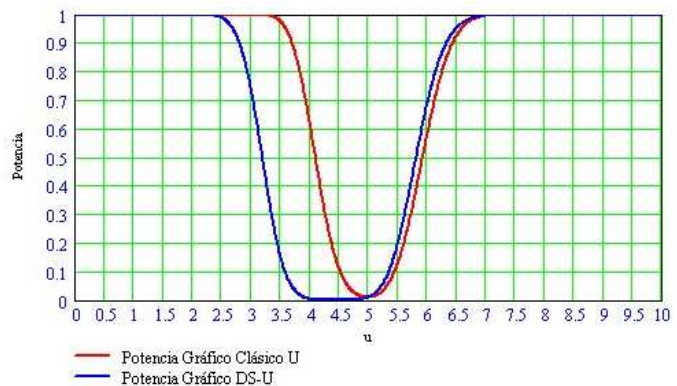

Fig. A.221: Curvas de potencia para $u_{1}=6,5$

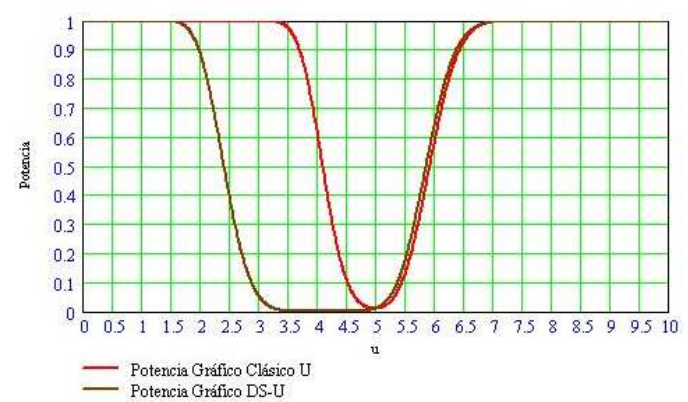

Fig. A.222: Curvas de potencia para $u_{1}=7,0$ 
APÉNDICE A. COMPARACIÓN DE LA POTENCIA DEL GRÁFICO U FRENTE A LA POTENCIA DEL GRÁFICO DS-U EN UN VALOR U

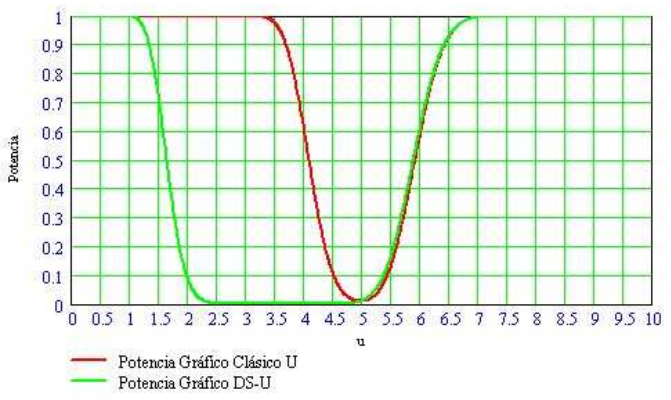

Fig. A.223: Curvas de potencia para $u_{1}=7,5$

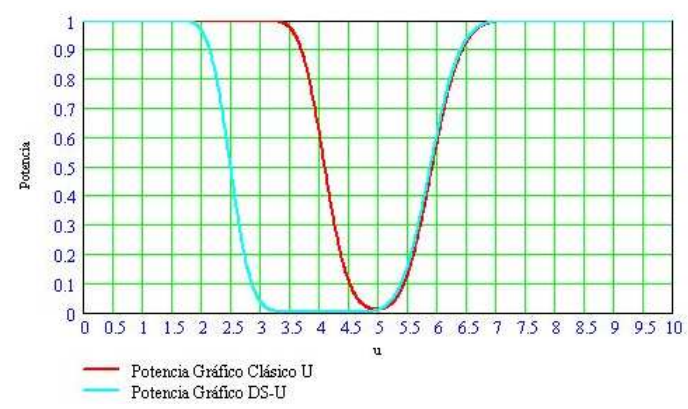

Fig. A.224: Curvas de potencia para $u_{1}=8,0$

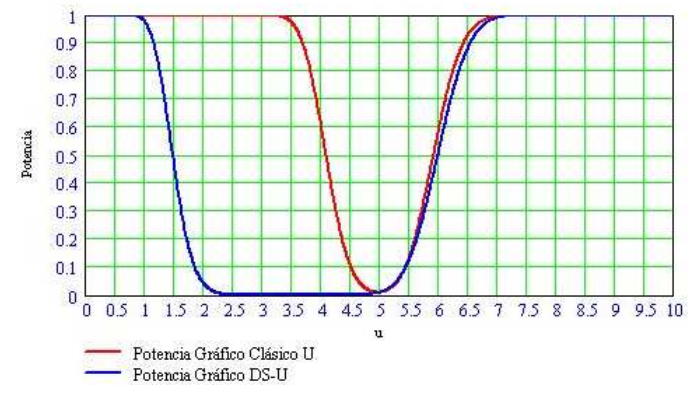

Fig. A.225: Curvas de potencia para $u_{1}=8,5$ 
APÉNDICE A. COMPARACIÓN DE LA POTENCIA DEL GRÁFICO U FRENTE A LA POTENCIA DEL GRÁFICO DS-U EN UN VALOR U

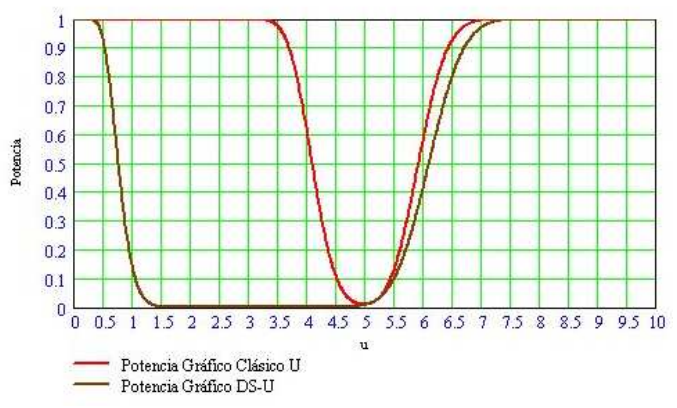

Fig. A.226: Curvas de potencia para $u_{1}=9,0$

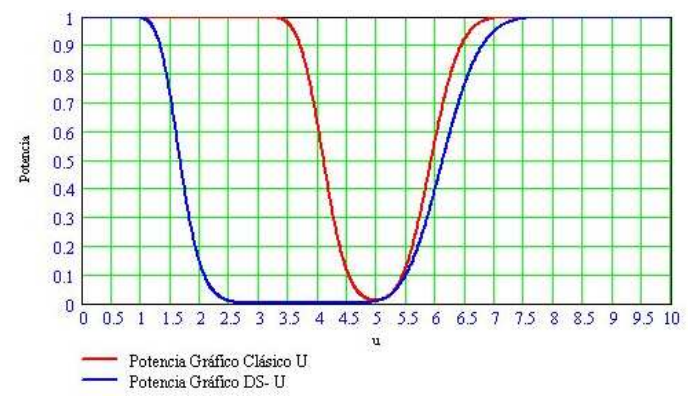

Fig. A.227: Curvas de potencia para $u_{1}=9,5$

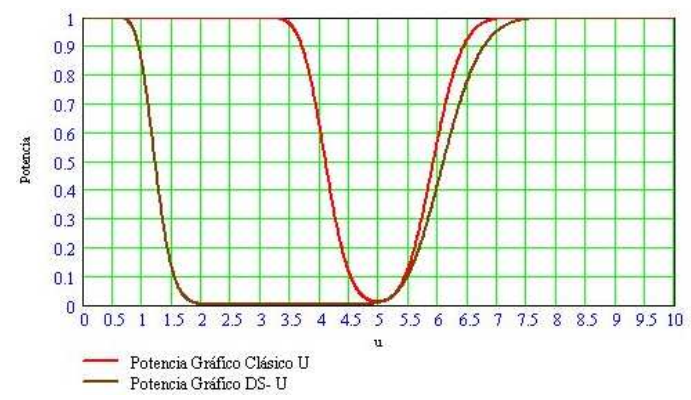

Fig. A.228: Curvas de potencia para $u_{1}=10,0$ 
APÉNDICE A. COMPARACIÓN DE LA POTENCIA DEL GRÁFICO U FRENTE A LA POTENCIA DEL GRÁFICO DS-U EN UN VALOR U

\begin{tabular}{|c||c|c|c|c|c|c||c|c|}
\hline$u_{1}$ & $L C I$ & $L A I$ & $L C I_{1}$ & $L A S$ & $L C S$ & $L C S_{1}$ & $n_{1}$ & $n_{2}$ \\
\hline 0.1 & 9.7 & 38.4 & 62.6 & 92.1 & 141.1 & 120.8 & 11 & 89 \\
0.5 & 30.3 & 37.3 & 56.0 & 78.1 & 128.9 & 86.9 & 12 & 35 \\
1.0 & 58.5 & 64.3 & 86.9 & 117.7 & 127.7 & 148.6 & 17 & 31 \\
1.5 & 27.0 & 46.5 & 68.1 & 46.8 & 118.9 & 134.7 & 13 & 19 \\
2.0 & 52.6 & 55.7 & 71.6 & 55.8 & 108.1 & 150.8 & 15 & 20 \\
2.5 & 7.3 & 90.5 & 96.4 & 146.6 & 157.4 & 148.8 & 23 & 21 \\
3.0 & 42.1 & 42.6 & 98.8 & 42.8 & 135.7 & 157.4 & 13 & 25 \\
3.5 & 55.2 & 55.6 & 94.1 & 55.8 & 109.2 & 157.6 & 16 & 24 \\
4.0 & 34.1 & 34.3 & 94.5 & 34.9 & 78.2 & 156.6 & 11 & 24 \\
4.5 & 20.5 & 82.7 & 76.0 & 146.9 & 152.2 & 150.2 & 21 & 91 \\
\hline 5.0 & 62.1 & 62.3 & 63.2 & 112.5 & 120.5 & 143.9 & 18 & 35 \\
\hline 5.5 & 76.4 & 76.8 & 99.4 & 126.1 & 146.4 & 147.3 & 23 & 26 \\
6.0 & 6.6 & 14.6 & 67.6 & 128.4 & 150.6 & 150.0 & 24 & 26 \\
6.5 & 51.5 & 72.8 & 97.2 & 120.1 & 141.8 & 158.2 & 22 & 28 \\
7.0 & 5.2 & 45.5 & 92.0 & 98.3 & 122.7 & 158.6 & 19 & 27 \\
7.5 & 9.2 & 51.7 & 43.0 & 94.4 & 121.9 & 155.6 & 19 & 26 \\
8.0 & 6.4 & 65.7 & 65.1 & 109.6 & 138.3 & 156.4 & 22 & 26 \\
8.5 & 16.6 & 29.3 & 71.6 & 92.5 & 124.7 & 151.6 & 20 & 24 \\
9.0 & 1.1 & 37.4 & 13.4 & 103.1 & 135.1 & 123.6 & 22 & 18 \\
9.5 & 25.4 & 27.0 & 34.5 & 31.8 & 159.1 & 146.6 & 16 & 24 \\
10.0 & 22.2 & 26.5 & 89.7 & 135.5 & 135.7 & 154.8 & 22 & 28 \\
\hline
\end{tabular}

Tabla A.19: Parámetros obtenidos en la comparación de la potencia para el nuevo gráfico $D S-U: u_{0}=5, \alpha_{t}=0.01, n=40$ 
APÉNDICE A. COMPARACIÓN DE LA POTENCIA DEL GRÁFICO U FRENTE A LA POTENCIA DEL GRÁFICO DS-U EN UN VALOR U

\begin{tabular}{|c||c|c||c|c|}
\hline$u_{1}$ & Pot $_{U}$ & Pot $_{D S-U}$ & Pot $_{D S-U}-$ Pot $_{U}$ & $\alpha_{\text {real }}$ \\
\hline .1 & 1 & 1 & 0 & $9.930780 \cdot 10^{-3}$ \\
0.5 & 1 & 1 & 0 & 0.011703 \\
1.0 & 1 & 1 & 0 & $8.036459 \cdot 10^{-3}$ \\
1.5 & 1 & 1 & 0.021145 & $2.287688 \cdot 10^{-3}$ \\
2.0 & 1 & 1 & $-3.130567 \cdot 10^{-10}$ & $4.734990 \cdot 10^{-3}$ \\
2.5 & 1 & 0.999972 & $-2.765182 \cdot 10^{-5}$ & $2.423571 \cdot 10^{-3}$ \\
3.0 & 0.999920 & 0.998711 & $-1.208934 \cdot 10^{-3}$ & 0.011234 \\
3.5 & 0.974283 & 0.934254 & -0.040029 & 0.011463 \\
4.0 & 0.613647 & 0.485522 & -0.128125 & 0.011782 \\
4.5 & 0.108118 & 0.106661 & $-1.456708 \cdot 10^{-3}$ & 0.011812 \\
\hline 5.0 & $9.857263 \cdot 10^{-3}$ & 0.011828 & $1.971010 \cdot 10^{-3}$ & 0.011828 \\
\hline 5.5 & 0.133524 & 0.198582 & 0.065058 & 0.011820 \\
6.0 & 0.585384 & 0.698959 & 0.113575 & 0.011782 \\
6.5 & 0.929183 & 0.956115 & 0.026933 & 0.011514 \\
7.0 & 0.996110 & 0.997003 & $8.928025 \cdot 10^{-4}$ & 0.011654 \\
7.5 & 0.999927 & 0.999926 & $-8.784777 \cdot 10^{-7}$ & 0.011770 \\
8.0 & 0.999999 & 1 & $3.050651 \cdot 10^{-7}$ & 0.010284 \\
8.5 & 1 & 1 & $-6.505040 \cdot 10^{-9}$ & 0.010870 \\
9.0 & 1 & 1 & $-1.078124 \cdot 10^{-9}$ & $9.401436 \cdot 10^{-3}$ \\
9.5 & 1 & 1 & $-2.942837 \cdot 10^{-9}$ & $9.333886 \cdot 10^{-3}$ \\
10.0 & 1 & 1 & $1.329714 \cdot 10^{-11}$ & $9.607826 \cdot 10^{-3}$ \\
\hline
\end{tabular}

Tabla A.20: Resultados para $u_{0}=5, \alpha_{\text {teórico }}=0.01$ y $n=40$ 
APÉNDICE A. COMPARACIÓN DE LA POTENCIA DEL GRÁFICO U FRENTE A LA POTENCIA DEL GRÁFICO DS-U EN UN VALOR U

A.11. Comparación de los gráficos de control $u$ y $\boldsymbol{D S}-\boldsymbol{U} \operatorname{con} n=40, u_{0}=5$ y $\alpha_{t}=0.05$ 
APÉNDICE A. COMPARACIÓN DE LA POTENCIA DEL GRÁFICO U FRENTE A LA POTENCIA DEL GRÁFICO DS-U EN UN VALOR U

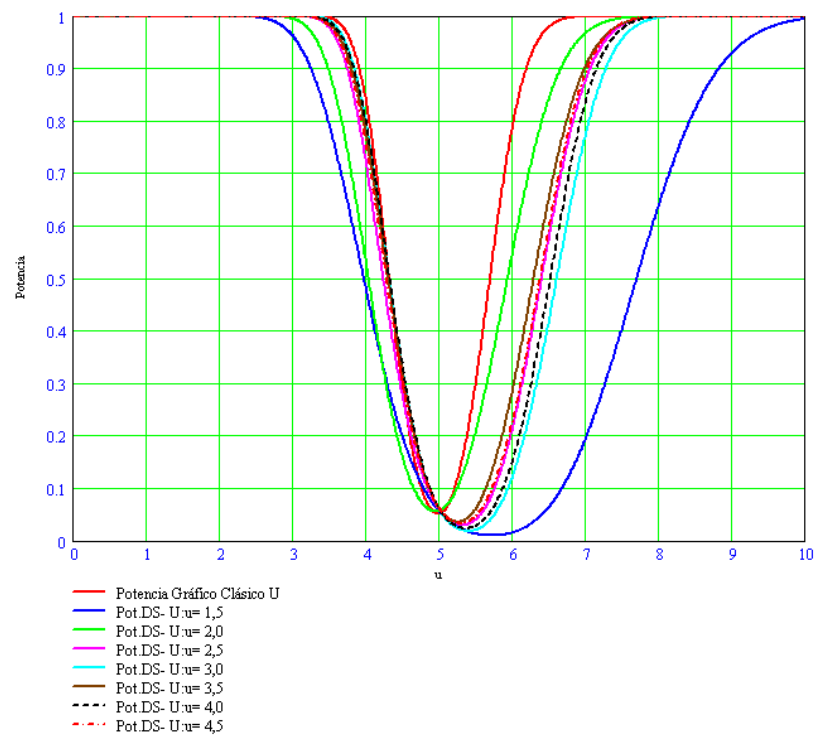

(a) $u_{1} \in[0.1,5.0]$

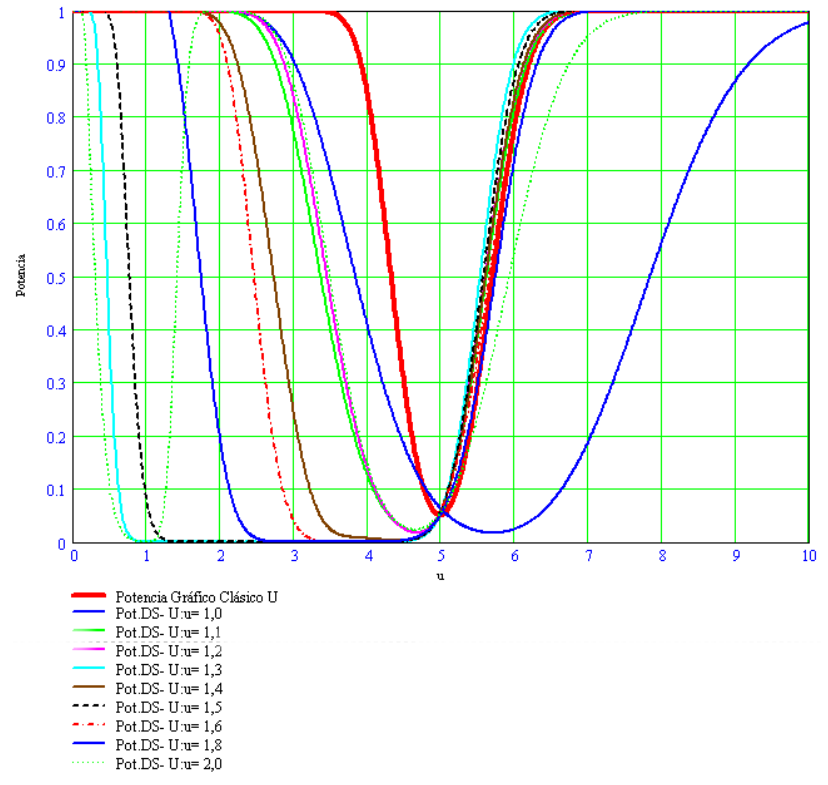

(b) $u_{1} \in[5.5,10.0]$

Fig. A.229: Curvas de potencia en distintos valores de $u_{1}$ 
APÉNDICE A. COMPARACIÓN DE LA POTENCIA DEL GRÁFICO U FRENTE A LA POTENCIA DEL GRÁFICO DS-U EN UN VALOR U

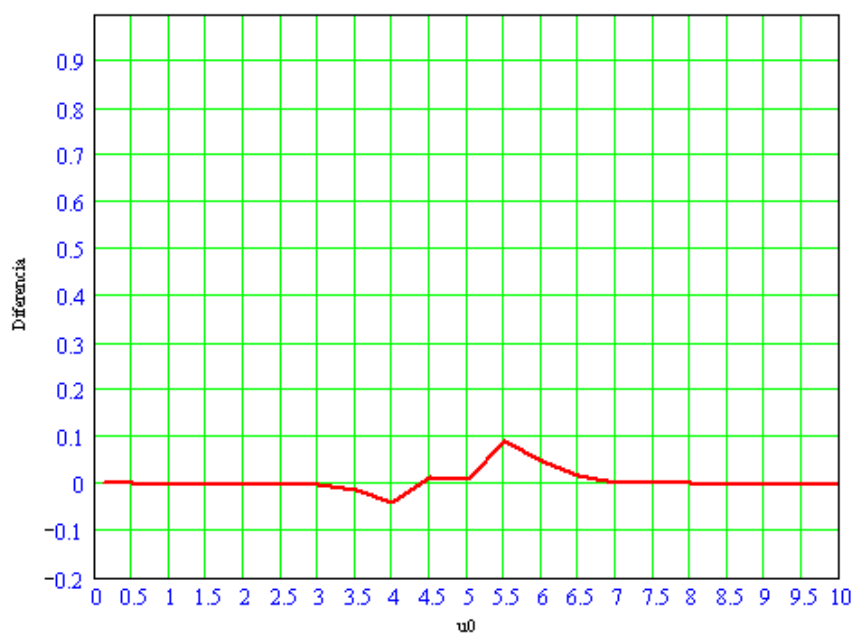

Fig. A.230: $\operatorname{Pot}_{D S-U}-\operatorname{Pot}_{u}$ para $n=40, u_{0}=5$ y $\alpha_{t}=0.05$

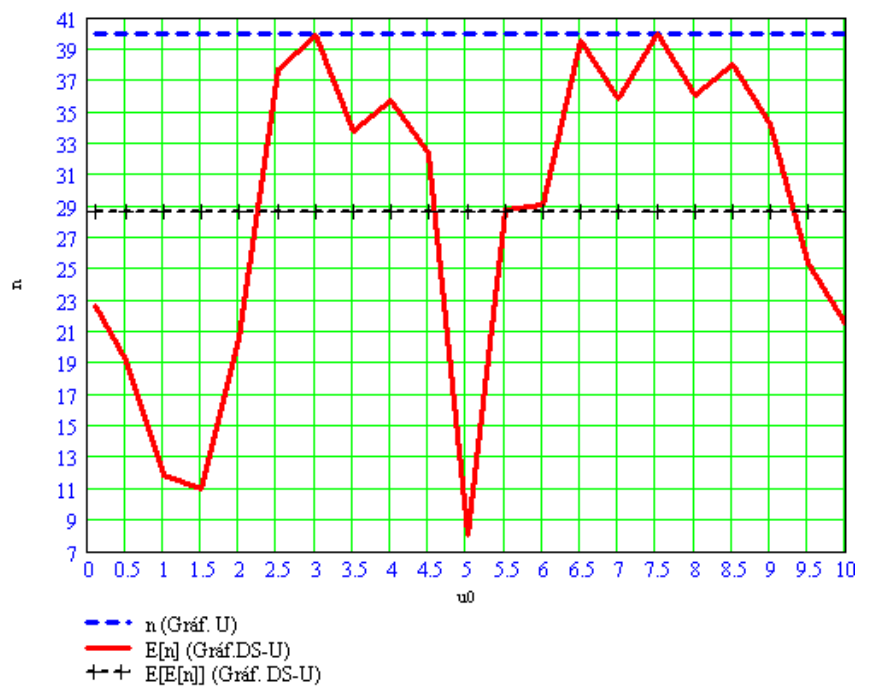

Fig. A.231: Tamaño medio de muestra en el gráfico $D S-U$ para $n=40, u_{0}=5$ y $\alpha_{t}=0.05$ 
APÉNDICE A. COMPARACIÓN DE LA POTENCIA DEL GRÁFICO U FRENTE A LA POTENCIA DEL GRÁFICO DS-U EN UN VALOR U

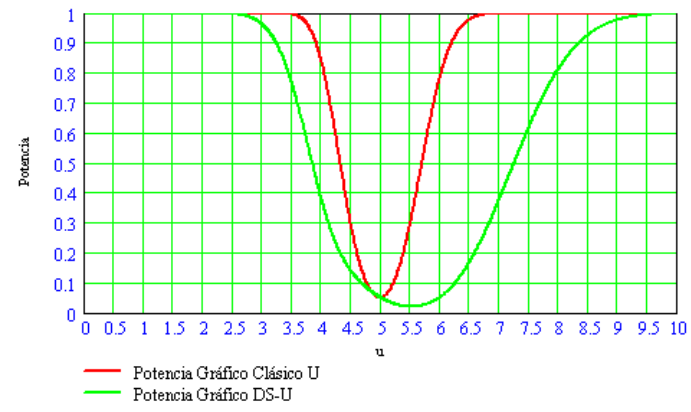

Fig. A.232: Curvas de potencia para $u_{1}=1,0$

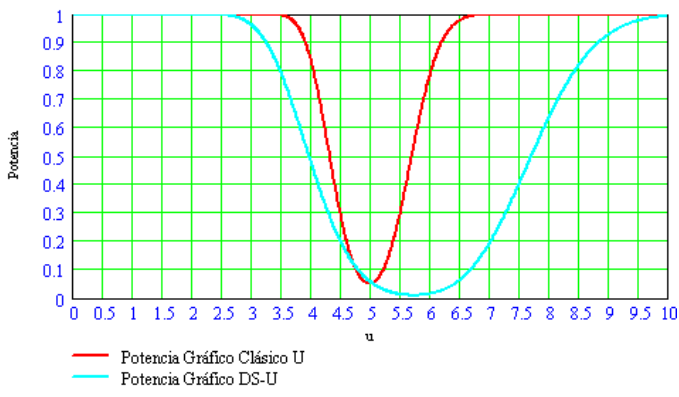

Fig. A.233: Curvas de potencia para $u_{1}=1,5$

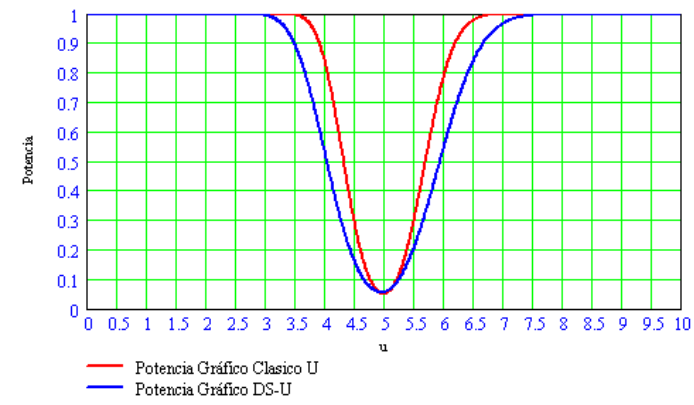

Fig. A.234: Curvas de potencia para $u_{1}=2,0$ 
APÉNDICE A. COMPARACIÓN DE LA POTENCIA DEL GRÁFICO U FRENTE A LA POTENCIA DEL GRÁFICO DS-U EN UN VALOR U

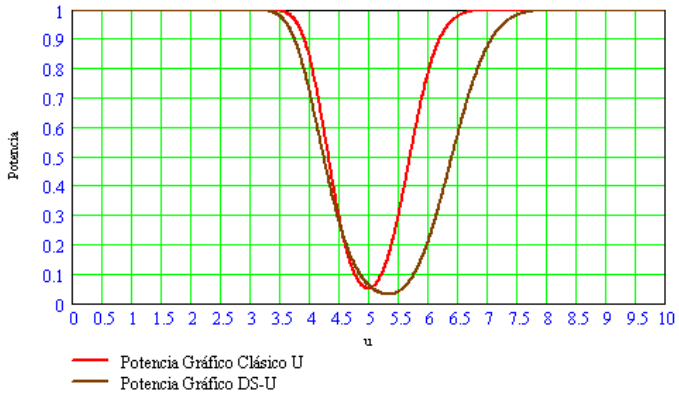

Fig. A.235: Curvas de potencia para $u_{1}=2,5$

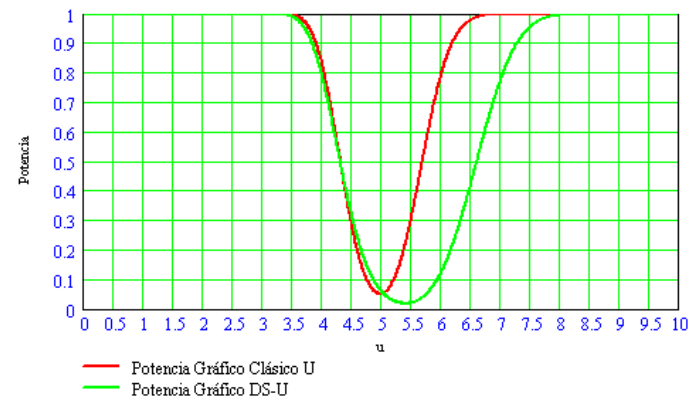

Fig. A.236: Curvas de potencia para $u_{1}=3,0$

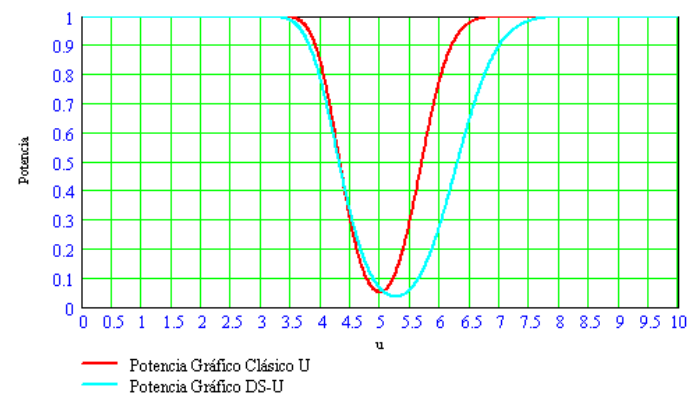

Fig. A.237: Curvas de potencia para $u_{1}=3,5$ 
APÉNDICE A. COMPARACIÓN DE LA POTENCIA DEL GRÁFICO U FRENTE A LA POTENCIA DEL GRÁFICO DS-U EN UN VALOR U

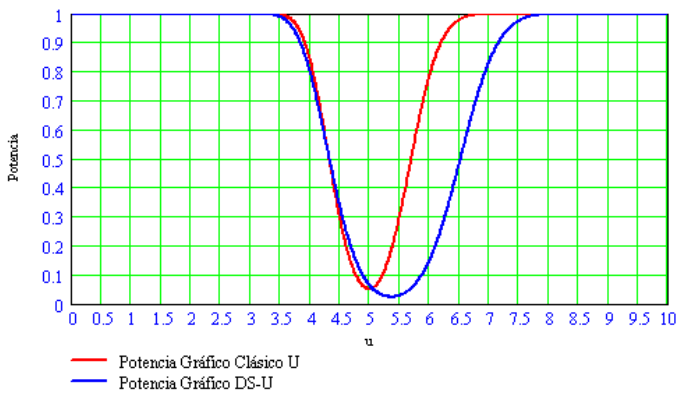

Fig. A.238: Curvas de potencia para $u_{1}=4,0$

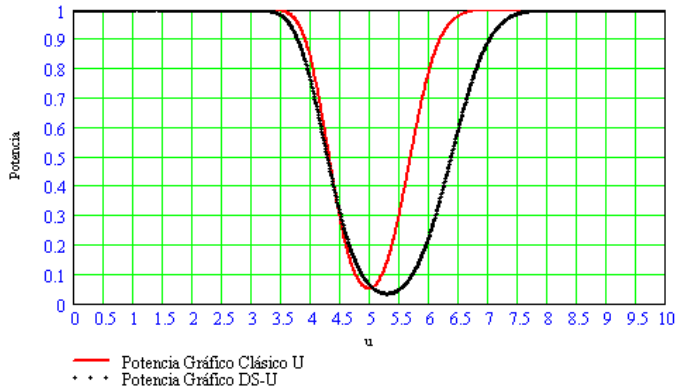

Fig. A.239: Curvas de potencia para $u_{1}=4,5$

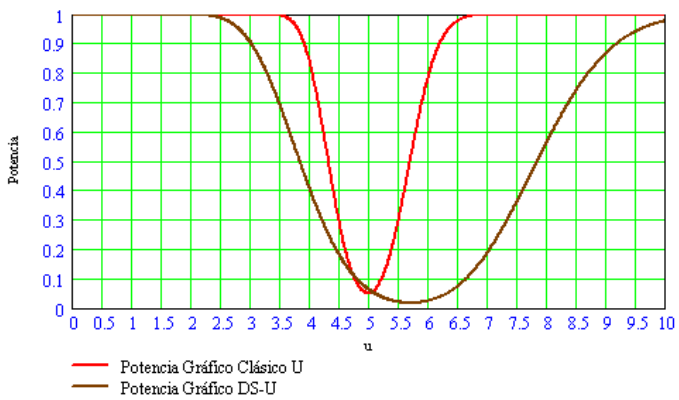

Fig. A.240: Curvas de potencia para $u_{1}=5,0$ 
APÉNDICE A. COMPARACIÓN DE LA POTENCIA DEL GRÁFICO U FRENTE A LA POTENCIA DEL GRÁFICO DS-U EN UN VALOR U

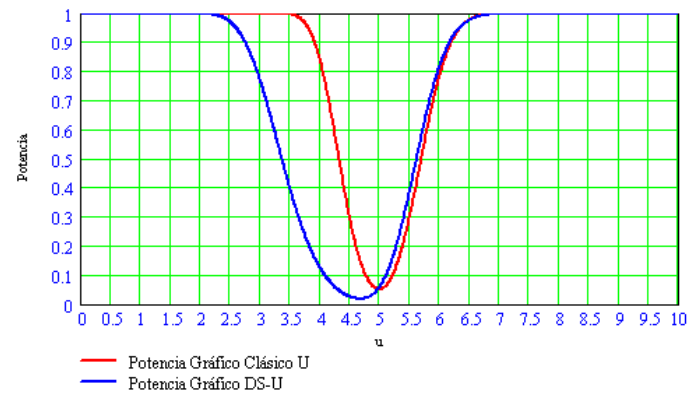

Fig. A.241: Curvas de potencia para $u_{1}=5,5$

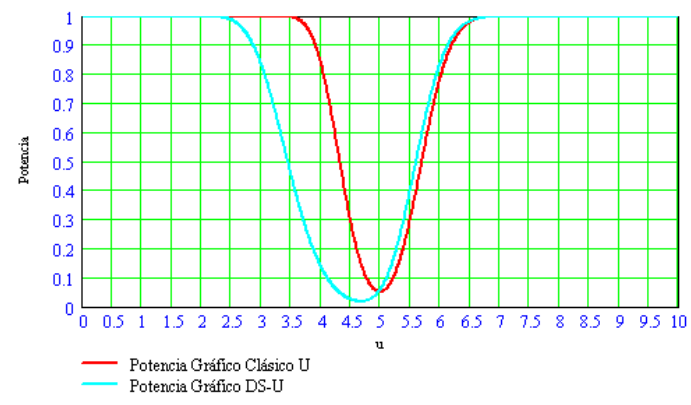

Fig. A.242: Curvas de potencia para $u_{1}=6,0$

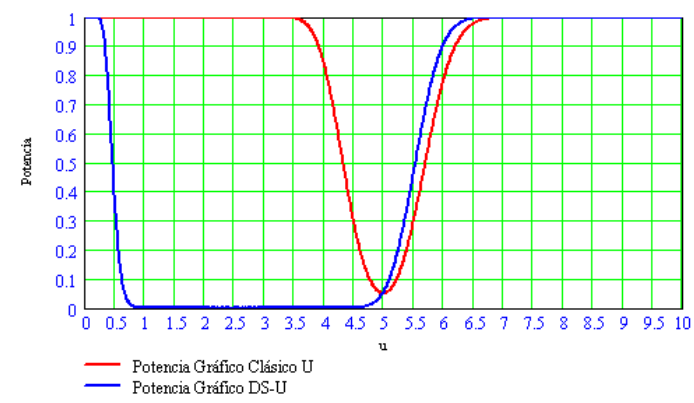

Fig. A.243: Curvas de potencia para $u_{1}=6,5$ 
APÉNDICE A. COMPARACIÓN DE LA POTENCIA DEL GRÁFICO U FRENTE A LA POTENCIA DEL GRÁFICO DS-U EN UN VALOR U

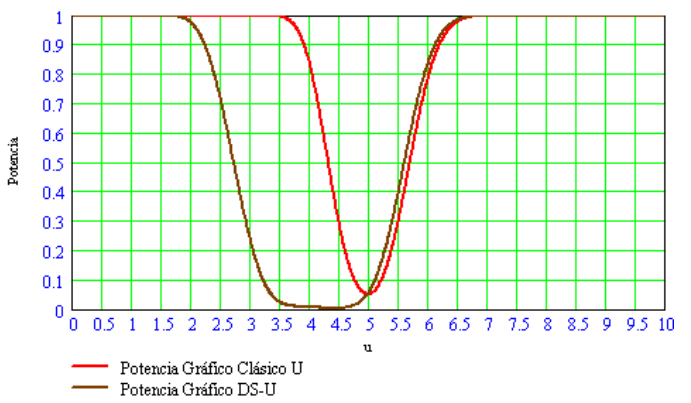

Fig. A.244: Curvas de potencia para $u_{1}=7,0$

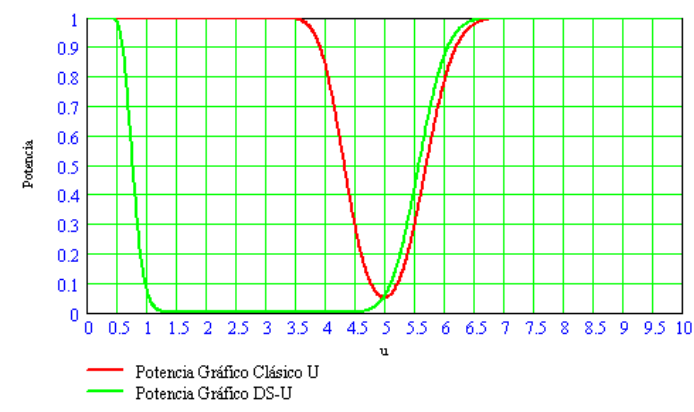

Fig. A.245: Curvas de potencia para $u_{1}=7,5$

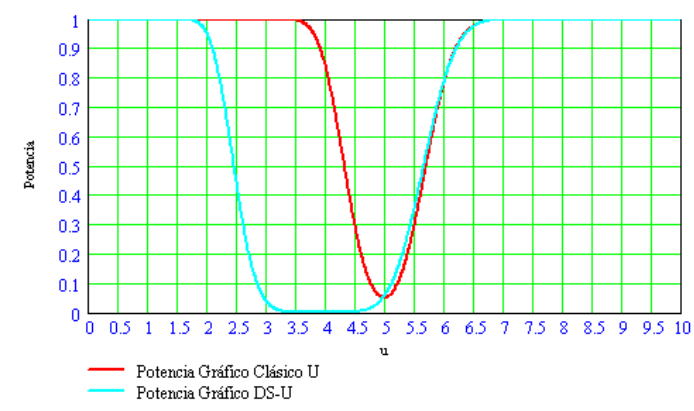

Fig. A.246: Curvas de potencia para $u_{1}=8,0$ 
APÉNDICE A. COMPARACIÓN DE LA POTENCIA DEL GRÁFICO U FRENTE A LA POTENCIA DEL GRÁFICO DS-U EN UN VALOR U

\begin{tabular}{|c||c|c|c|c|c|c||c|c|}
\hline$u_{1}$ & $L C I$ & $L A I$ & $L C I_{1}$ & $L A S$ & $L C S$ & $L C S_{1}$ & $n_{1}$ & $n_{2}$ \\
\hline 0.1 & 2.7 & 54.5 & 95.3 & 99.2 & 123.7 & 158.3 & 17 & 92 \\
0.5 & 33.0 & 50.6 & 35.5 & 89.5 & 124.9 & 151.5 & 15 & 80 \\
1.0 & 40.9 & 43.1 & 96.6 & 79.9 & 83.8 & 116.4 & 11 & 25 \\
1.5 & 43.1 & 43.3 & 58.8 & 84.2 & 131.4 & 106.5 & 11 & 23 \\
2.0 & 32.5 & 80.1 & 97.7 & 118.5 & 132.1 & 153.9 & 20 & 10 \\
2.5 & 61.2 & 61.3 & 91.6 & 61.6 & 120.8 & 153.1 & 15 & 24 \\
3.0 & 79.3 & 79.8 & 85.4 & 79.9 & 148.5 & 145.4 & 19 & 22 \\
3.5 & 38.1 & 38.2 & 97.1 & 38.7 & 157.0 & 144.5 & 11 & 23 \\
4.0 & 47.1 & 47.5 & 97.1 & 47.8 & 89.7 & 156.9 & 13 & 23 \\
4.5 & 45.2 & 45.7 & 87.0 & 45.9 & 82.8 & 138.3 & 12 & 21 \\
\hline 5.0 & 30.1 & 30.8 & 54.4 & 63.0 & 77.6 & 75.2 & 8 & 16 \\
\hline 5.5 & 43.2 & 43.7 & 99.9 & 63.8 & 86.3 & 155.3 & 13 & 28 \\
6.0 & 51.8 & 51.9 & 96.7 & 74.2 & 95.7 & 155.1 & 15 & 28 \\
6.5 & 9.6 & 23.9 & 11.4 & 135.1 & 157.5 & 157.0 & 27 & 28 \\
7.0 & 1.5 & 43.7 & 96.8 & 74.7 & 99.8 & 158.0 & 16 & 28 \\
7.5 & 14.9 & 72.5 & 18.2 & 110.5 & 135.6 & 154.6 & 23 & 27 \\
8.0 & 17.2 & 68.6 & 80.5 & 142.7 & 158.7 & 151.9 & 28 & 23 \\
8.5 & 14.3 & 18.0 & 63.0 & 47.9 & 95.0 & 142.2 & 16 & 23 \\
9.0 & 21.0 & 34.9 & 58.1 & 95.8 & 116.5 & 144.3 & 20 & 23 \\
9.5 & 10.6 & 41.6 & 84.2 & 122.0 & 130.0 & 142.0 & 21 & 90 \\
10.0 & 4.2 & 55.8 & 14.9 & 94.8 & 142.3 & 130.0 & 16 & 93 \\
\hline
\end{tabular}

Tabla A.21: Parámetros obtenidos en la optimización de la potencia para el nuevo gráfico $D S-U: u_{0}=5, \alpha_{t}=0.05, n=40$ 
APÉNDICE A. COMPARACIÓN DE LA POTENCIA DEL GRÁFICO U FRENTE A LA POTENCIA DEL GRÁFICO DS-U EN UN VALOR U

\begin{tabular}{|c||c|c||c|c|}
\hline$u_{1}$ & Pot $_{U}$ & Pot $_{D S-U}$ & Pot $_{D S-U}-$ Pot $_{U}$ & $\alpha_{\text {real }}$ \\
\hline 0.1 & 1 & 1 & 0 & 0.060957 \\
0.5 & 1 & 1 & $1.445510 \cdot 10^{-13}$ & 0.051595 \\
1.0 & 1 & 1 & $-5.495604 \cdot 10^{-14}$ & 0.049294 \\
1.5 & 1 & 1 & $-1.493583 \cdot 10^{-8}$ & 0.056381 \\
2.0 & 1 & 1 & $-8.279263 \cdot 10^{-9}$ & 0.057553 \\
2.5 & 1 & 1 & $-1.146471 \cdot 10^{-8}$ & 0.060756 \\
3.0 & 0.999997 & 0.999976 & $-2.065734 \cdot 10^{-5}$ & 0.060726 \\
3.5 & 0.996130 & 0.984314 & -0.011816 & 0.061766 \\
4.0 & 0.838611 & 0.796420 & -0.042192 & 0.061734 \\
4.5 & 0.291329 & 0.303570 & 0.012241 & 0.061752 \\
\hline 5.0 & 0.051681 & 0.062017 & 0.010337 & 0.062017 \\
\hline 5.5 & 0.304081 & 0.392672 & 0.088591 & 0.062002 \\
6.0 & 0.789065 & 0.839045 & 0.049980 & 0.061972 \\
6.5 & 0.979785 & 0.996400 & 0.016615 & 0.060844 \\
7.0 & 0.999389 & 0.999575 & $1.855729 \cdot 10^{-4}$ & 0.060590 \\
7.5 & 0.999994 & 0.999999 & $5.757012 \cdot 10^{-6}$ & 0.061252 \\
8.0 & 1 & 1 & $8.875061 \cdot 10^{-9}$ & 0.061533 \\
8.5 & 1 & 1 & $-4.520412 \cdot 10^{-9}$ & 0.050810 \\
9.0 & 1 & 1 & $-2.893352 \cdot 10^{-12}$ & 0.054630 \\
9.5 & 1 & 1 & $-1.381245 \cdot 10^{-9}$ & 0.056315 \\
10.0 & 1 & 1 & $-1.095667 \cdot 10^{-8}$ & 0.057487 \\
\hline
\end{tabular}

Tabla A.22: Resultados para $n=40, u_{0}=5$ y $\alpha_{t}=0.05$ 
APÉNDICE A. COMPARACIÓN DE LA POTENCIA DEL GRÁFICO U FRENTE A LA POTENCIA DEL GRÁFICO DS-U EN UN VALOR U

\begin{tabular}{|c||c|c|c|c|c|c||c|c|}
\hline$u_{1}$ & $L C I$ & $L A I$ & $L C I_{1}$ & $L A S$ & $L C S$ & $L C S_{1}$ & $n_{1}$ & $n_{2}$ \\
\hline 0.1 & 2.7 & 54.5 & 95.3 & 99.2 & 123.7 & 158.3 & 17 & 92 \\
0.5 & 33.0 & 50.6 & 35.5 & 89.5 & 124.9 & 151.5 & 15 & 80 \\
1.0 & 40.9 & 43.1 & 96.6 & 79.9 & 83.8 & 116.4 & 11 & 25 \\
1.5 & 43.1 & 43.3 & 58.8 & 84.2 & 131.4 & 106.5 & 11 & 23 \\
2.0 & 32.5 & 80.1 & 97.7 & 118.5 & 132.1 & 153.9 & 20 & 10 \\
2.5 & 61.2 & 61.3 & 91.6 & 61.6 & 120.8 & 153.1 & 15 & 24 \\
3.0 & 79.3 & 79.8 & 85.4 & 79.9 & 148.5 & 145.4 & 19 & 22 \\
3.5 & 38.1 & 38.2 & 97.1 & 38.7 & 157.0 & 144.5 & 11 & 23 \\
4.0 & 47.1 & 47.5 & 97.1 & 47.8 & 89.7 & 156.9 & 13 & 23 \\
4.5 & 45.2 & 45.7 & 87.0 & 45.9 & 82.8 & 138.3 & 12 & 21 \\
\hline 5.0 & 30.1 & 30.8 & 54.4 & 63.0 & 77.6 & 75.2 & 8 & 16 \\
\hline 5.5 & 43.2 & 43.7 & 99.9 & 63.8 & 86.3 & 155.3 & 13 & 28 \\
6.0 & 51.8 & 51.9 & 96.7 & 74.2 & 95.7 & 155.1 & 15 & 28 \\
6.5 & 9.6 & 23.9 & 11.4 & 135.1 & 157.5 & 157.0 & 27 & 28 \\
7.0 & 1.5 & 43.7 & 96.8 & 74.7 & 99.8 & 158.0 & 16 & 28 \\
7.5 & 14.9 & 72.5 & 18.2 & 110.5 & 135.6 & 154.6 & 23 & 27 \\
8.0 & 17.2 & 68.6 & 80.5 & 142.7 & 158.7 & 151.9 & 28 & 23 \\
8.5 & 14.3 & 18.0 & 63.0 & 47.9 & 95.0 & 142.2 & 16 & 23 \\
9.0 & 21.0 & 34.9 & 58.1 & 95.8 & 116.5 & 144.3 & 20 & 23 \\
9.5 & 10.6 & 41.6 & 84.2 & 122.0 & 130.0 & 142.0 & 21 & 90 \\
10.0 & 4.2 & 55.8 & 14.9 & 94.8 & 142.3 & 130.0 & 16 & 93 \\
\hline
\end{tabular}

Tabla A.23: Parámetros obtenidos en la comparación de la potencia para el nuevo gráfico $D S-U: u_{0}=5, \alpha_{t}=0.05, n=40$. 
APÉNDICE A. COMPARACIÓN DE LA POTENCIA DEL GRÁFICO U FRENTE A LA POTENCIA DEL GRÁFICO DS-U EN UN VALOR U

\begin{tabular}{|c||c|c||c|c|}
\hline$u_{1}$ & Pot $_{U}$ & Pot $_{D S-U}$ & Pot $_{D S-U}-$ Pot $_{U}$ & $\alpha_{\text {real }}$ \\
\hline 0.1 & 1 & 1 & 0 & 0.060957 \\
0.5 & 1 & 1 & $1.445510 \cdot 10^{-13}$ & 0.051595 \\
1.0 & 1 & 1 & $-5.495604 \cdot 10^{-14}$ & 0.049294 \\
1.5 & 1 & 1 & $-1.493583 \cdot 10^{-8}$ & 0.056381 \\
2.0 & 1 & 1 & $-8.279263 \cdot 10^{-9}$ & 0.057553 \\
2.5 & 1 & 1 & $-1.146471 \cdot 10^{-8}$ & 0.060756 \\
3.0 & 0.999997 & 0.999976 & $-2.065734 \cdot 10^{-5}$ & 0.060726 \\
3.5 & 0.996130 & 0.984314 & -0.011816 & 0.061766 \\
4.0 & 0.838611 & 0.796420 & -0.042192 & 0.061734 \\
4.5 & 0.291329 & 0.303570 & 0.012241 & 0.061752 \\
\hline 5.0 & 0.051681 & 0.062017 & 0.010337 & 0.062017 \\
\hline 5.5 & 0.304081 & 0.392672 & 0.088591 & 0.062002 \\
6.0 & 0.789065 & 0.839045 & 0.049980 & 0.061972 \\
6.5 & 0.979785 & 0.996400 & 0.016615 & 0.060844 \\
7.0 & 0.999389 & 0.999575 & $1.855729 \cdot 10^{-4}$ & 0.060590 \\
7.5 & 0.999994 & 0.999999 & $5.757012 \cdot 10^{-6}$ & 0.061252 \\
8.0 & 1 & 1 & $8.875061 \cdot 10^{-9}$ & 0.061533 \\
8.5 & 1 & 1 & $-4.520412 \cdot 10^{-9}$ & 0.050810 \\
9.0 & 1 & 1 & $-2.893352 \cdot 10^{-12}$ & 0.054630 \\
9.5 & 1 & 1 & $-1.381245 \cdot 10^{-9}$ & 0.056315 \\
10.0 & 1 & 1 & $-1.095667 \cdot 10^{-8}$ & 0.057487 \\
\hline
\end{tabular}

Tabla A.24: Resultados para $u_{0}=5, \alpha_{\text {teórico }}=0.05$ y $n=40$ 
APÉNDICE A. COMPARACIÓN DE LA POTENCIA DEL GRÁFICO U FRENTE A LA POTENCIA DEL GRÁFICO DS-U EN UN VALOR U

A.12. Comparación de los gráficos de control $u$ y $\boldsymbol{D S}-\boldsymbol{U} \operatorname{con} n=40, u_{0}=5$ y $\alpha_{t}=0.0027$ 
APÉNDICE A. COMPARACIÓN DE LA POTENCIA DEL GRÁFICO U FRENTE A LA POTENCIA DEL GRÁFICO DS-U EN UN VALOR U

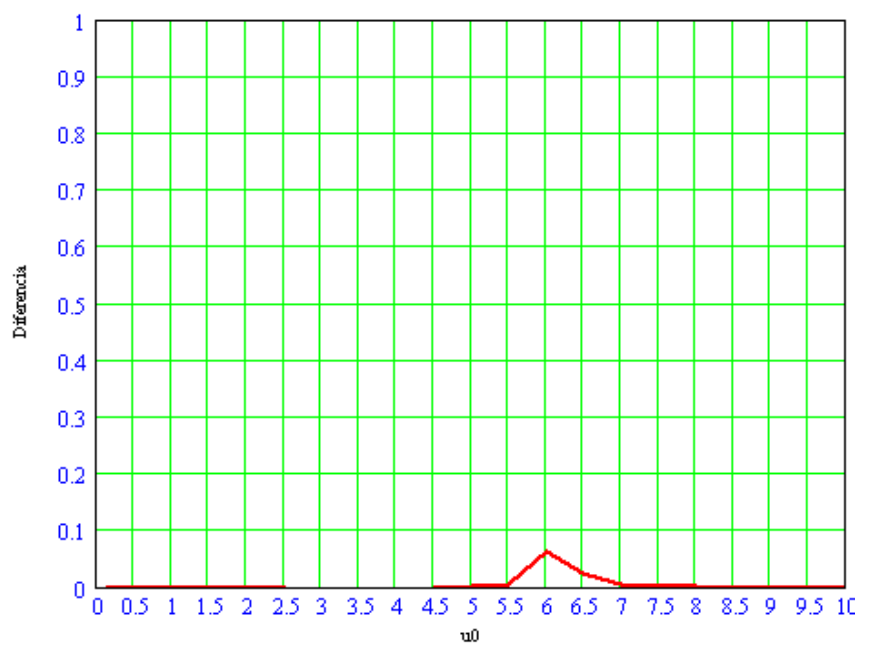

Fig. A.247: $\operatorname{Pot}_{D S-U}-\operatorname{Pot}_{u}$ para $n=40, u_{0}=5$ y $\alpha_{t}=0.0027$

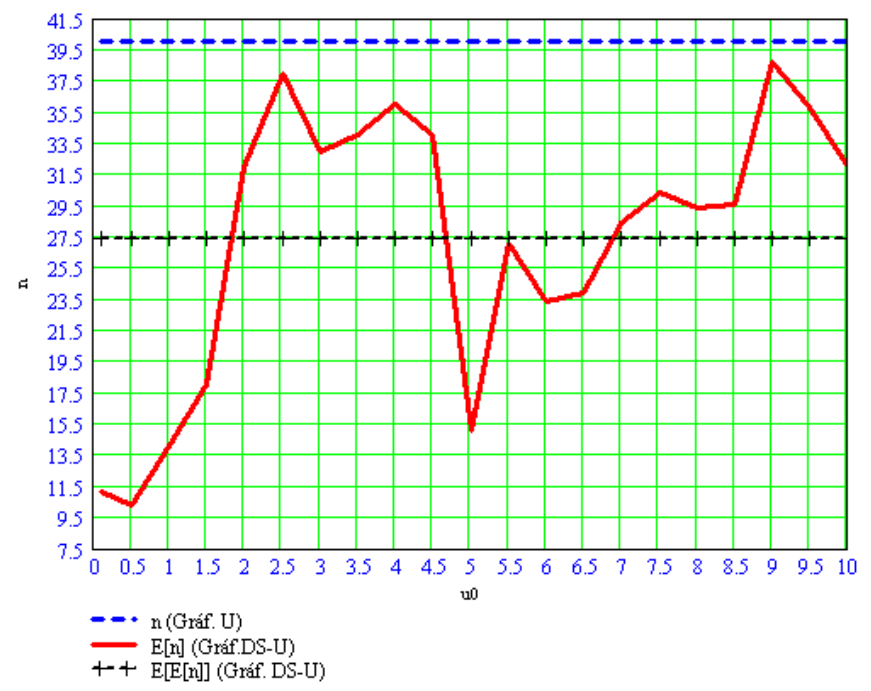

Fig. A.248: Tamaño medio de muestra en el gráfico $D S-U$ para $n=40, u_{0}=5$ y $\alpha_{t}=0.0027$ 
APÉNDICE A. COMPARACIÓN DE LA POTENCIA DEL GRÁFICO U FRENTE A LA POTENCIA DEL GRÁFICO DS-U EN UN VALOR U

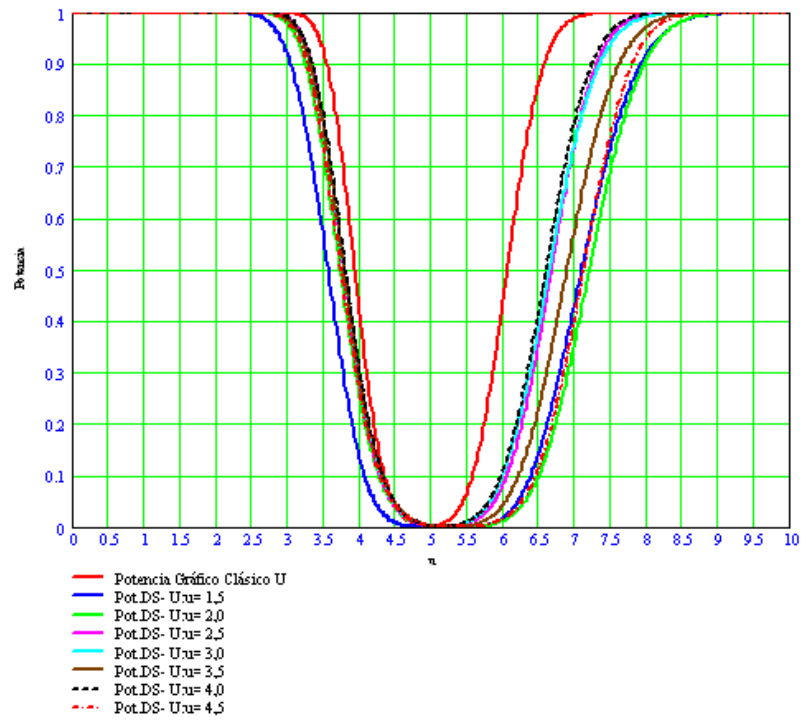

Fig. A.249: Curvas de potencia en un valor $u_{1}$ para $u \in[0.1,1.0]$

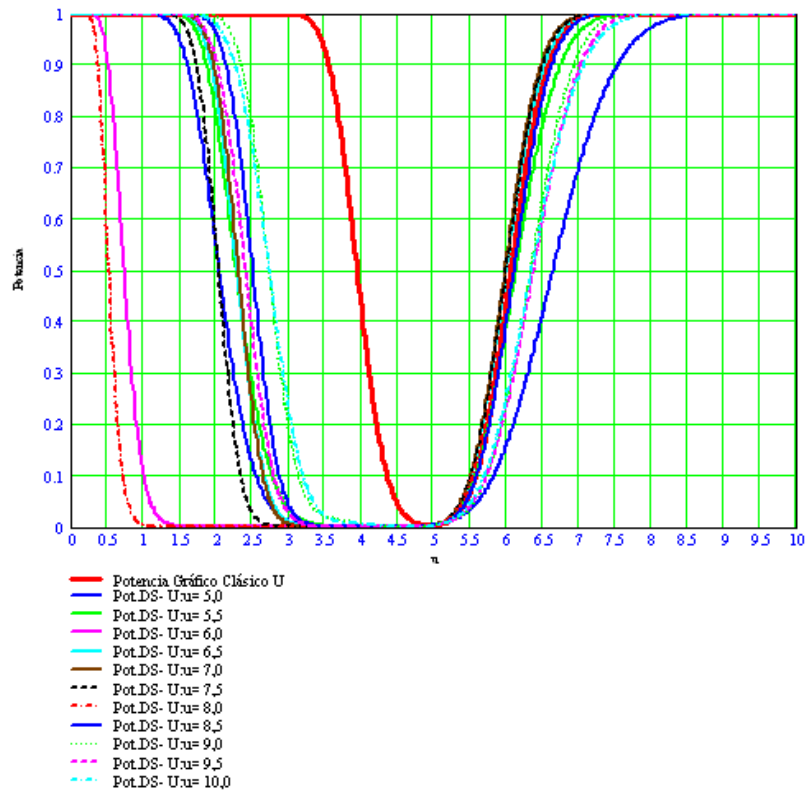

Fig. A.250: Curvas de potencia en un valor $u_{1}$ para $u \in[1.1,2.0]$ 
APÉNDICE A. COMPARACIÓN DE LA POTENCIA DEL GRÁFICO U FRENTE A LA POTENCIA DEL GRÁFICO DS-U EN UN VALOR U

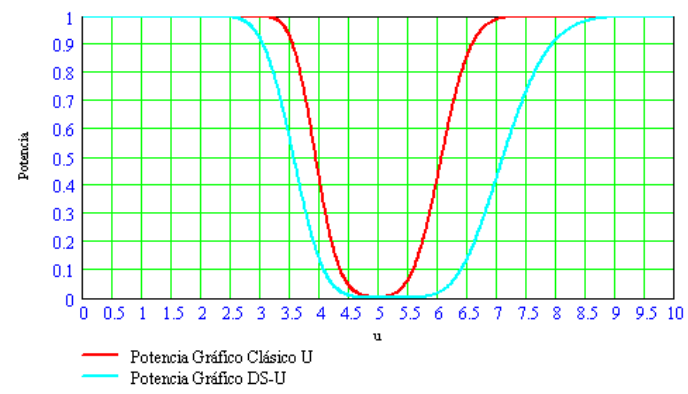

Fig. A.251: Curvas de potencia para $u_{1}=1,5$

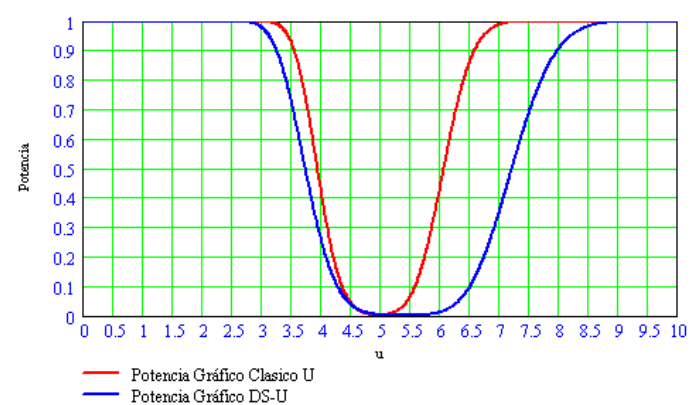

Fig. A.252: Curvas de potencia para $u_{1}=2,0$

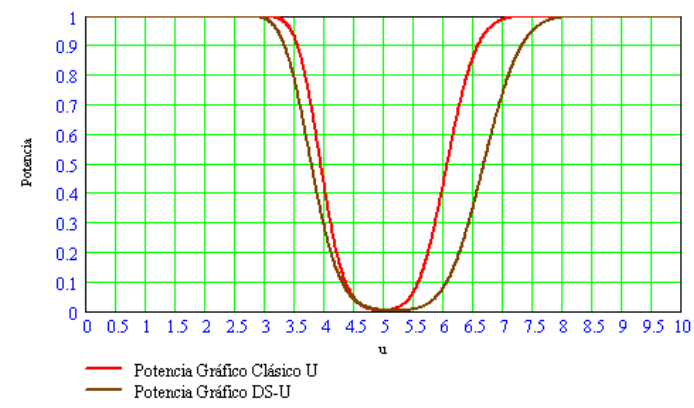

Fig. A.253: Curvas de potencia para $u_{1}=2,5$ 
APÉNDICE A. COMPARACIÓN DE LA POTENCIA DEL GRÁFICO U FRENTE A LA POTENCIA DEL GRÁFICO DS-U EN UN VALOR U

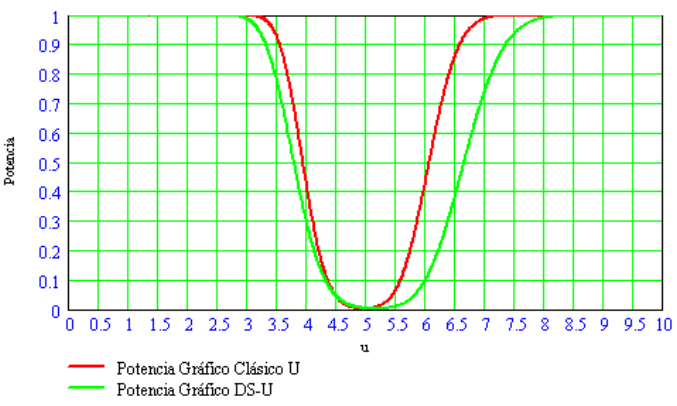

Fig. A.254: Curvas de potencia para $u_{1}=3,0$

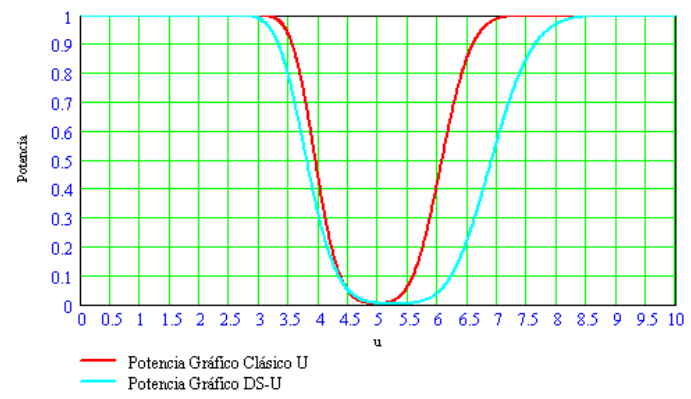

Fig. A.255: Curvas de potencia para $u_{1}=3,5$

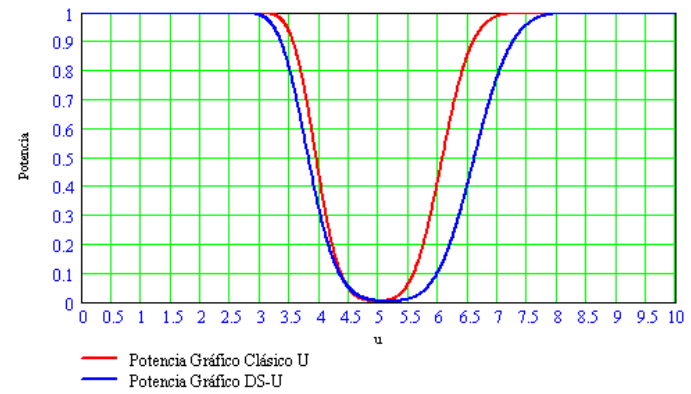

Fig. A.256: Curvas de potencia para $u_{1}=4,0$ 
APÉNDICE A. COMPARACIÓN DE LA POTENCIA DEL GRÁFICO U FRENTE A LA POTENCIA DEL GRÁFICO DS-U EN UN VALOR U

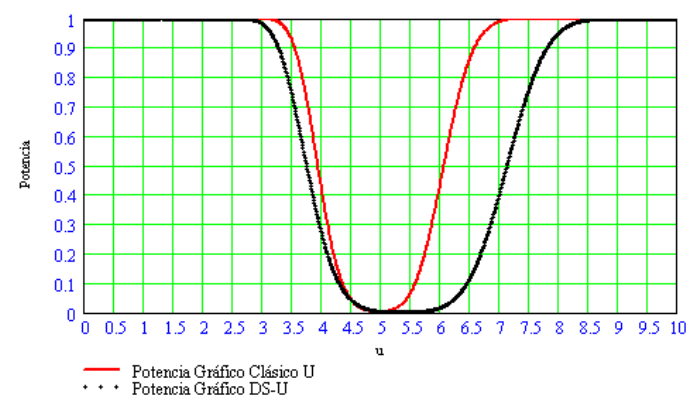

Fig. A.257: Curvas de potencia para $u_{1}=4,5$

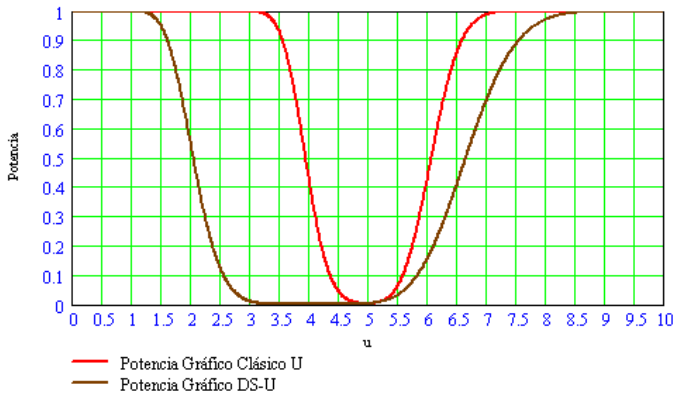

Fig. A.258: Curvas de potencia para $u_{1}=5,0$

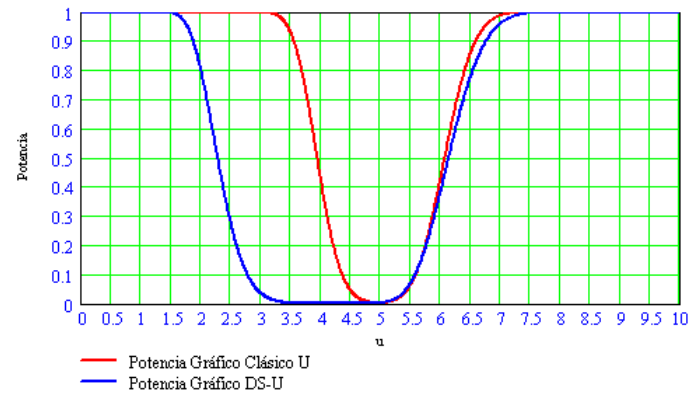

Fig. A.259: Curvas de potencia para $u_{1}=5,5$ 
APÉNDICE A. COMPARACIÓN DE LA POTENCIA DEL GRÁFICO U FRENTE A LA POTENCIA DEL GRÁFICO DS-U EN UN VALOR U

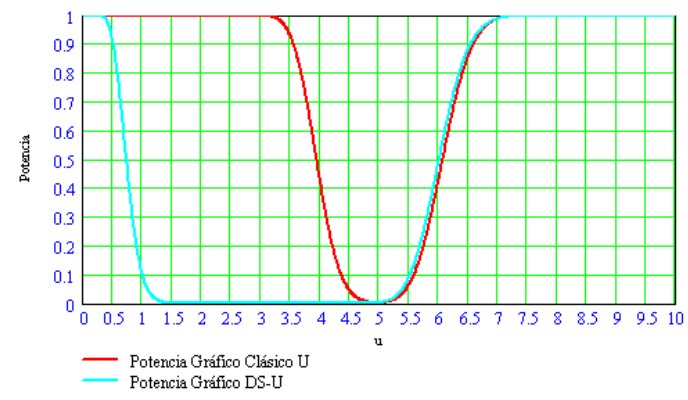

Fig. A.260: Curvas de potencia para $u_{1}=6,0$

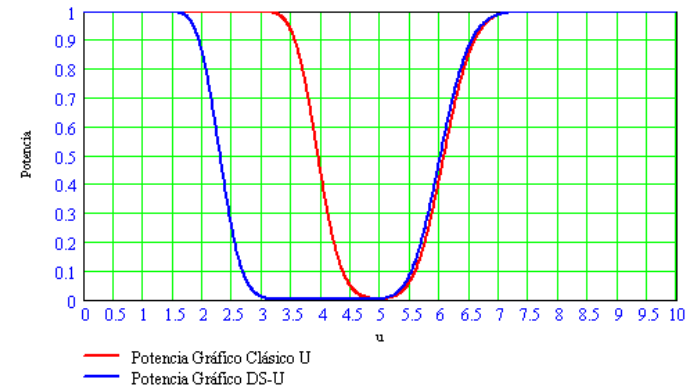

Fig. A.261: Curvas de potencia para $u_{1}=6,5$

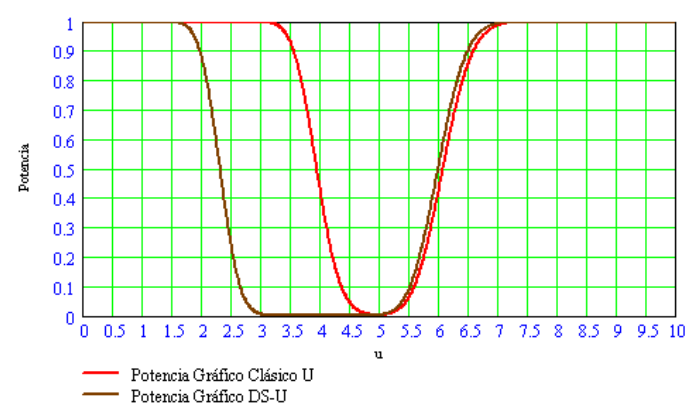

Fig. A.262: Curvas de potencia para $u_{1}=7,0$ 
APÉNDICE A. COMPARACIÓN DE LA POTENCIA DEL GRÁFICO U FRENTE A LA POTENCIA DEL GRÁFICO DS-U EN UN VALOR U

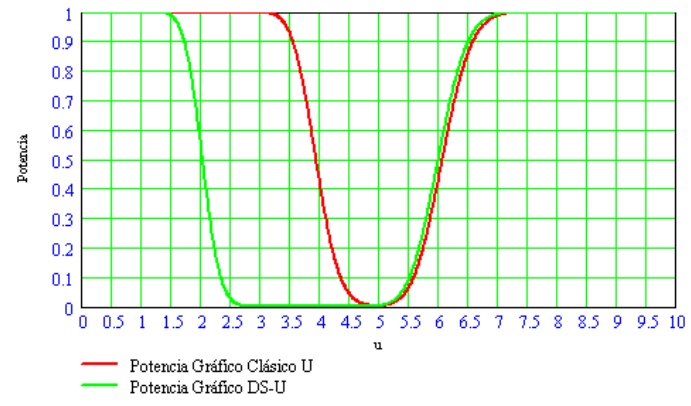

Fig. A.263: Curvas de potencia para $u_{1}=7,5$

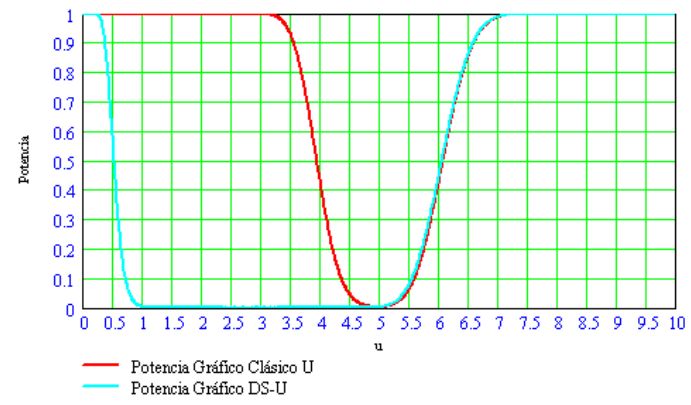

Fig. A.264: Curvas de potencia para $u_{1}=8,0$

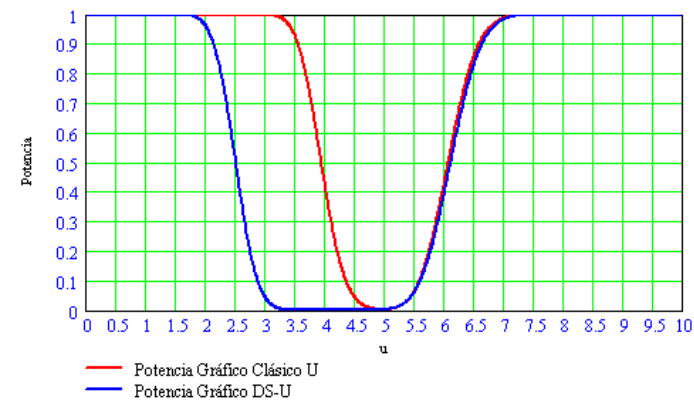

Fig. A.265: Curvas de potencia para $u_{1}=8,5$ 
APÉNDICE A. COMPARACIÓN DE LA POTENCIA DEL GRÁFICO U FRENTE A LA POTENCIA DEL GRÁFICO DS-U EN UN VALOR U

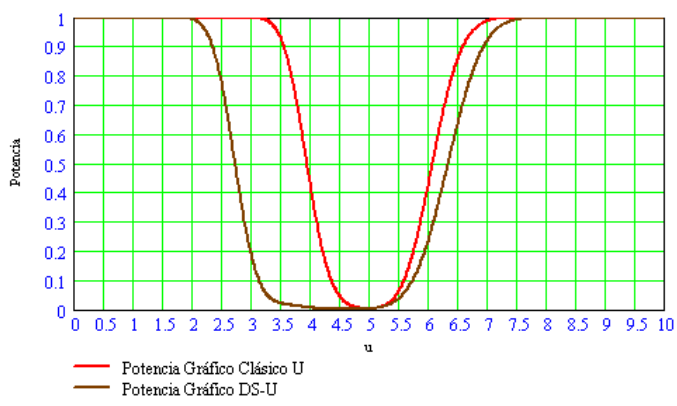

Fig. A.266: Curvas de potencia para $u_{1}=9,0$

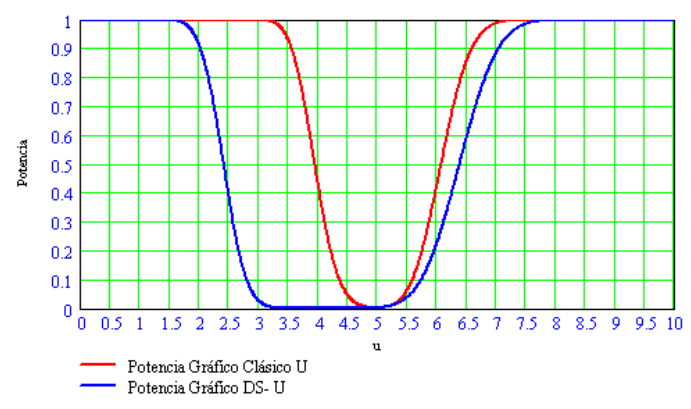

Fig. A.267: Curvas de potencia para $u_{1}=9,5$ 
APÉNDICE A. COMPARACIÓN DE LA POTENCIA DEL GRÁFICO U FRENTE A LA POTENCIA DEL GRÁFICO DS-U EN UN VALOR U

\begin{tabular}{|c||c|c|c|c|c|c||c|c|}
\hline$u_{1}$ & $L C I$ & $L A I$ & $L C I_{1}$ & $L A S$ & $L C S$ & $L C S_{1}$ & $n_{1}$ & $n_{2}$ \\
\hline 0.1 & 5.4 & 35.5 & 44.5 & 82.4 & 114.5 & 120.1 & 11 & 100 \\
0.5 & 10.5 & 31.7 & 92.7 & 75.5 & 156.9 & 103.0 & 10 & 94 \\
1.0 & 36.1 & 47.6 & 97.7 & 101.2 & 106.8 & 116.4 & 14 & 58 \\
1.5 & 53.6 & 64.6 & 97.2 & 127.9 & 151.1 & 136.0 & 18 & 23 \\
2.0 & 27.3 & 27.7 & 81.5 & 28.0 & 137.1 & 158.7 & 10 & 22 \\
2.5 & 47.1 & 47.4 & 85.8 & 48.0 & 112.0 & 155.3 & 15 & 23 \\
3.0 & 23.3 & 23.6 & 90.6 & 24.0 & 138.7 & 159.0 & 9 & 24 \\
3.5 & 31.3 & 31.6 & 86.3 & 31.7 & 126.5 & 158.7 & 11 & 23 \\
4.0 & 35.3 & 35.4 & 90.7 & 35.6 & 92.0 & 159.5 & 12 & 24 \\
4.5 & 43.5 & 43.9 & 74.0 & 44.0 & 106.9 & 147.9 & 14 & 20 \\
\hline 5.0 & 24.4 & 30.6 & 87.1 & 99.9 & 108.2 & 105.2 & 15 & 25 \\
\hline 5.5 & 38.5 & 41.6 & 44.5 & 87.3 & 137.8 & 158.6 & 17 & 26 \\
6.0 & 9.2 & 14.1 & 98.5 & 111.3 & 133.2 & 159.5 & 20 & 27 \\
6.5 & 5.8 & 64.6 & 59.7 & 117.4 & 139.8 & 153.4 & 21 & 26 \\
7.0 & 0.8 & 56.2 & 69.1 & 130.2 & 154.1 & 156.8 & 24 & 26 \\
7.5 & 20.2 & 50.0 & 60.0 & 127.1 & 155.6 & 157.3 & 24 & 26 \\
8.0 & 6.7 & 37.3 & 12.8 & 115.7 & 143.8 & 153.9 & 22 & 25 \\
8.5 & 0.4 & 70.3 & 58.1 & 127.3 & 152.4 & 146.7 & 24 & 23 \\
9.0 & 10.7 & 60.9 & 53.6 & 83.1 & 140.4 & 118.9 & 21 & 18 \\
9.5 & 5.2 & 62.7 & 46.3 & 103.6 & 140.4 & 142.3 & 22 & 19 \\
10.0 & 5.7 & 41.0 & 71.6 & 69.0 & 146.0 & 139.3 & 15 & 22 \\
\hline
\end{tabular}

Tabla A.25: Parámetros obtenidos en la comparación de la potencia para el nuevo gráfico $D S-U: u_{0}=5, \alpha_{t}=0.0027, n=40$. 
APÉNDICE A. COMPARACIÓN DE LA POTENCIA DEL GRÁFICO U FRENTE A LA POTENCIA DEL GRÁFICO DS-U EN UN VALOR U

\begin{tabular}{|c||c|c||c|c|}
\hline$u_{1}$ & Pot $_{u}$ & Pot $_{D S-U}$ & Pot $_{D S-U}-$ Pot $_{u}$ & $\alpha_{\text {real }}$ \\
\hline 0.1 & 1 & 1 & 0 & $2.899532 \cdot 10^{-3}$ \\
0.5 & 1 & 1 & $-2.905487 \cdot 10^{-11}$ & $3.058252 \cdot 10^{-3}$ \\
1.0 & 1 & 1 & $-1.223799 \cdot 10^{-12}$ & $2.483076 \cdot 10^{-3}$ \\
1.5 & 1 & 1 & $-4.186984 \cdot 10^{-10}$ & $2.018981 \cdot 10^{-4}$ \\
2.0 & 1 & 1 & $-1.044923 \cdot 10^{-8}$ & $2.587657 \cdot 10^{-3}$ \\
2.5 & 1 & 0.999985 & $-1.484919 \cdot 10^{-5}$ & $2.612841 \cdot 10^{-3}$ \\
3.0 & 0.999480 & 0.987177 & -0.012303 & $3.065375 \cdot 10^{-3}$ \\
3.5 & 0.928338 & 0.783186 & -0.145153 & $3.220032 \cdot 10^{-3}$ \\
4.0 & 0.426638 & 0.313212 & -0.113426 & $3.216107 \cdot 10^{-3}$ \\
4.5 & 0.044454 & 0.042381 & $-2.072313 \cdot 10^{-3}$ & $3.230283 \cdot 10^{-3}$ \\
\hline 5.0 & $2.693050 \cdot 10^{-3}$ & $3.231658 \cdot 10^{-3}$ & $5.386080 \cdot 10^{-4}$ & $3.231658 \cdot 10^{-3}$ \\
\hline 5.5 & 0.066399 & 0.072158 & $5.758958 \cdot 10^{-3}$ & $2.920633 \cdot 10^{-3}$ \\
6.0 & 0.431782 & 0.493575 & 0.061793 & $3.198749 \cdot 10^{-3}$ \\
6.5 & 0.861564 & 0.886401 & 0.024837 & $3.085996 \cdot 10^{-3}$ \\
7.0 & 0.988811 & 0.994651 & $5.839458 \cdot 10^{-3}$ & $3.192411 \cdot 10^{-3}$ \\
7.5 & 0.999693 & 0.999891 & $1.984443 \cdot 10^{-4}$ & $3.225328 \cdot 10^{-3}$ \\
8.0 & 0.999997 & 0.999998 & $6.572934 \cdot 10^{-7}$ & $3.054594 \cdot 10^{-3}$ \\
8.5 & 1 & 1 & $-2.343406 \cdot 10^{-9}$ & $2.674341 \cdot 10^{-3}$ \\
9.0 & 1 & 1 & $-1.985203 \cdot 10^{-8}$ & $2.441902 \cdot 10^{-3}$ \\
9.5 & 1 & 1 & $-4.266070 \cdot 10^{-10}$ & $2.538023 \cdot 10^{-3}$ \\
10.0 & 1 & $-1.224704 \cdot 10^{-9}$ & $2.594759 \cdot 10^{-3}$ \\
\hline
\end{tabular}

Tabla A.26: Resultados para $u_{0}=5, \alpha_{\text {teórico }}=0.0027$ y $n=40$ 
APÉNDICE A. COMPARACIÓN DE LA POTENCIA DEL GRÁFICO U FRENTE A LA POTENCIA DEL GRÁFICO DS-U EN UN VALOR U

A.13. Comparación de los gráficos de control $u$ y $D S-U$ en función del tamaño de muestra $n$, fijando $u_{0} \mathbf{y} \alpha$.

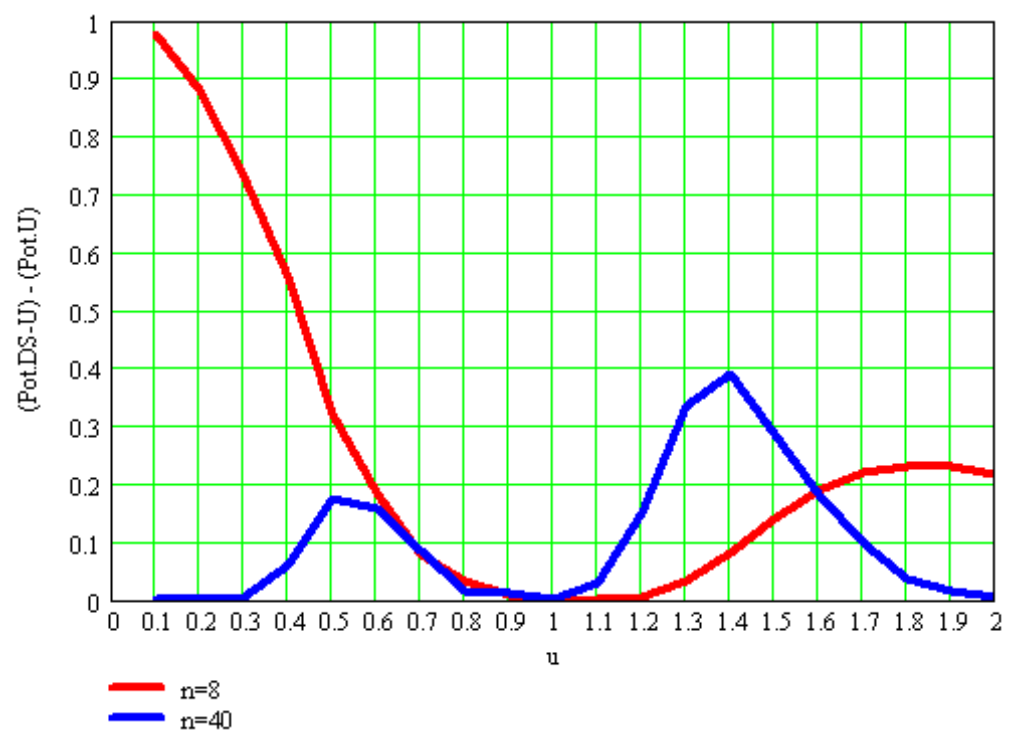

Fig. A.268: $\operatorname{Pot}_{D S-U}-$ Pot $_{u}$ para $u_{0}=1$ y $\alpha=0.0027$ 
APÉNDICE A. COMPARACIÓN DE LA POTENCIA DEL GRÁFICO U FRENTE A LA POTENCIA DEL GRÁFICO DS-U EN UN VALOR U

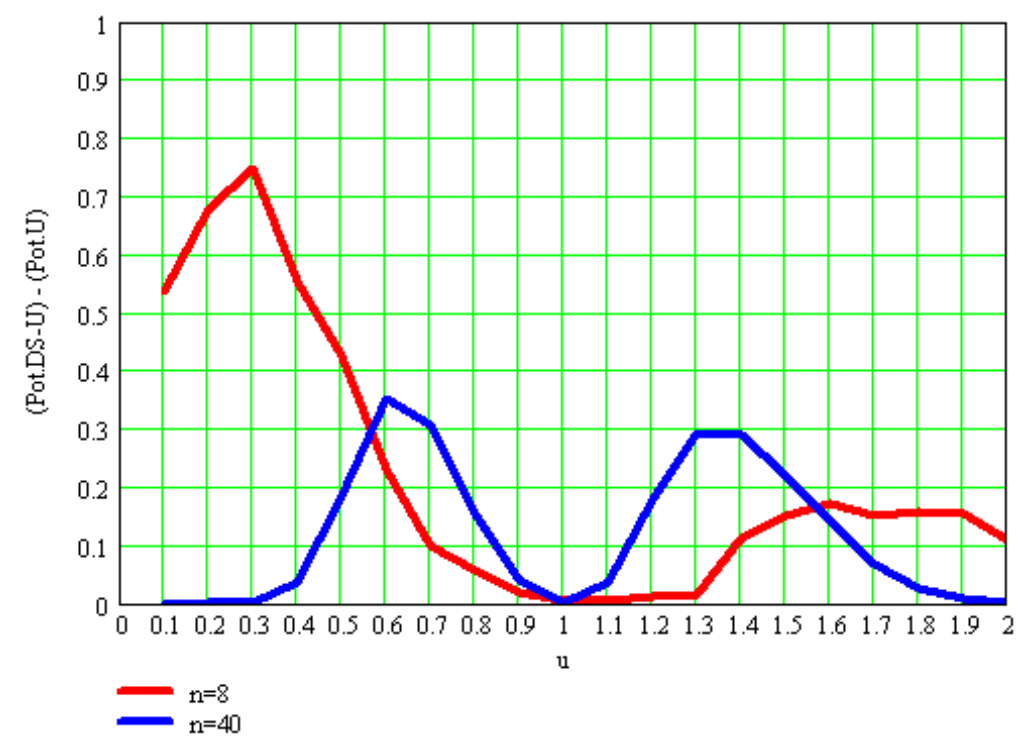

Fig. A.269: $\operatorname{Pot}_{D S-U}-\operatorname{Pot}_{u}$ para $u_{0}=1$ y $\alpha=0.01$

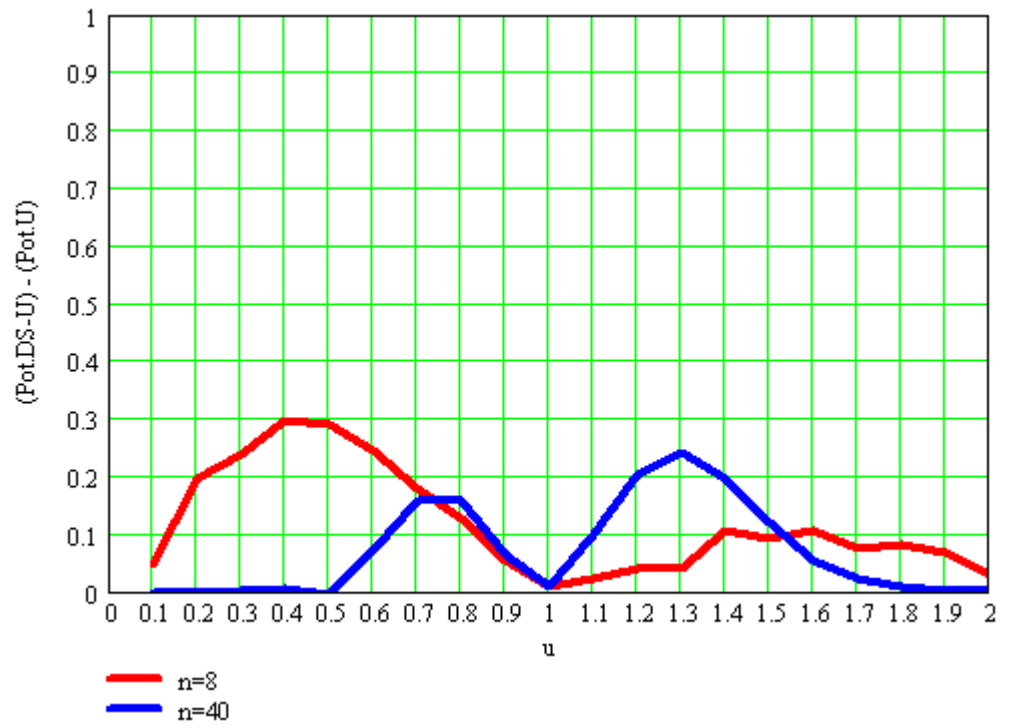

Fig. A.270: $\operatorname{Pot}_{D S-U}-\operatorname{Pot}_{u}$ para $u_{0}=1$ y $\alpha=0.05$ 
APÉNDICE A. COMPARACIÓN DE LA POTENCIA DEL GRÁFICO U FRENTE A LA POTENCIA DEL GRÁFICO DS-U EN UN VALOR U

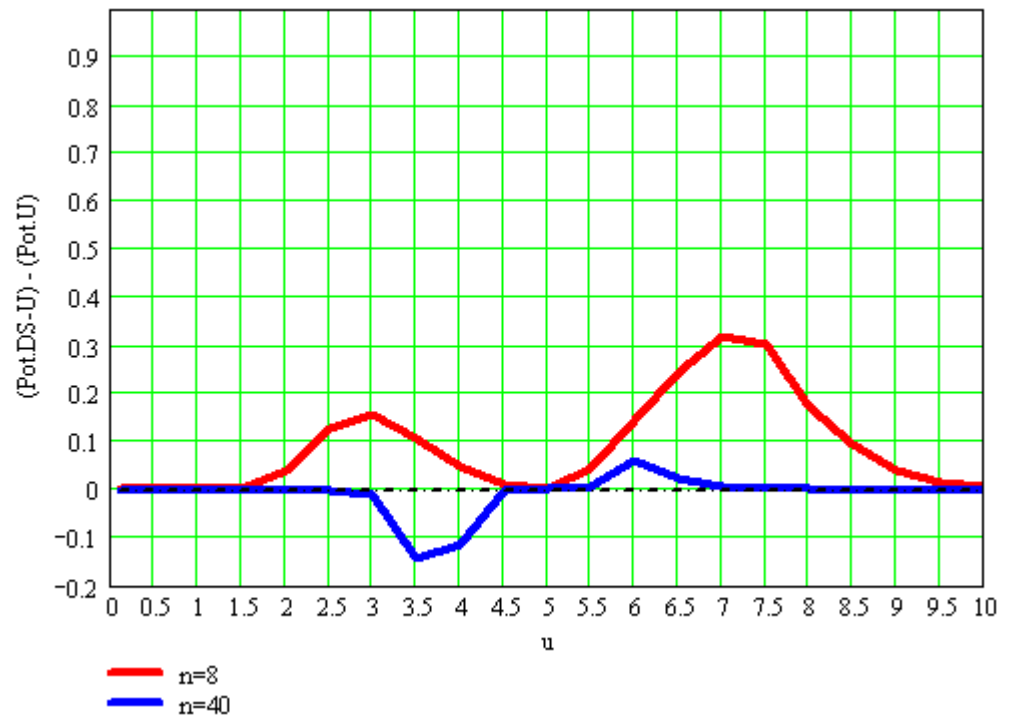

Fig. A.271: Pot $_{D S-U}-$ Pot $_{u}$ para $u_{0}=5$ y $\alpha=0.0027$

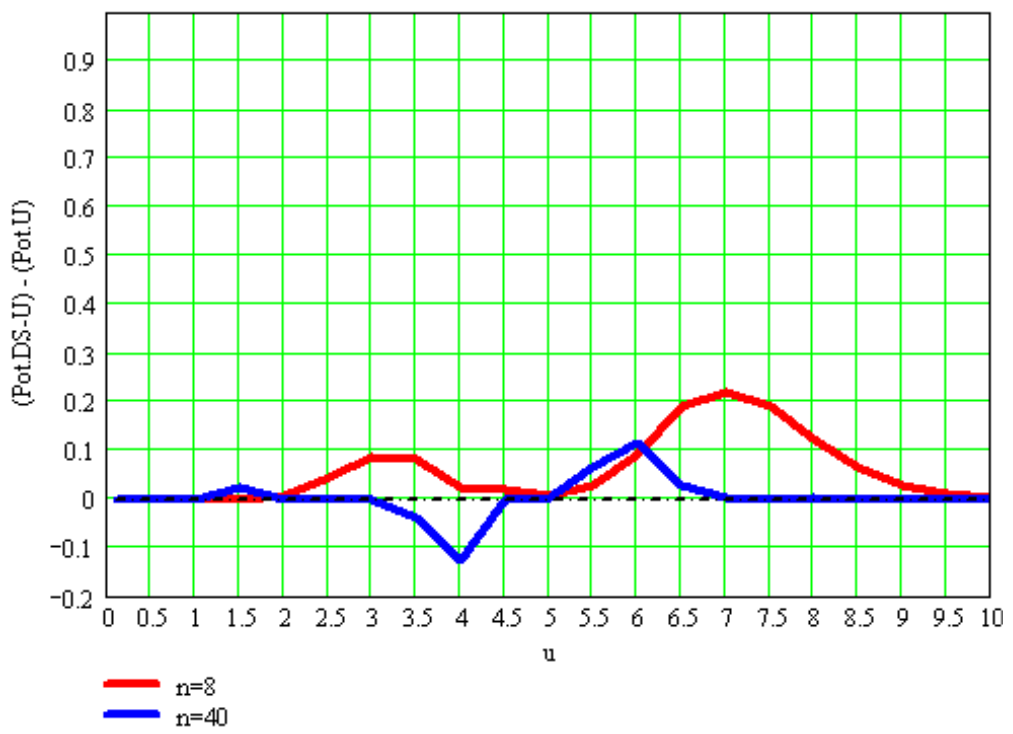

Fig. A.272: $\operatorname{Pot}_{D S-U}-P_{0} t_{u}$ para $u_{0}=5$ y $\alpha=0.01$ 
APÉNDICE A. COMPARACIÓN DE LA POTENCIA DEL GRÁFICO U FRENTE A LA POTENCIA DEL GRÁFICO DS-U EN UN VALOR U

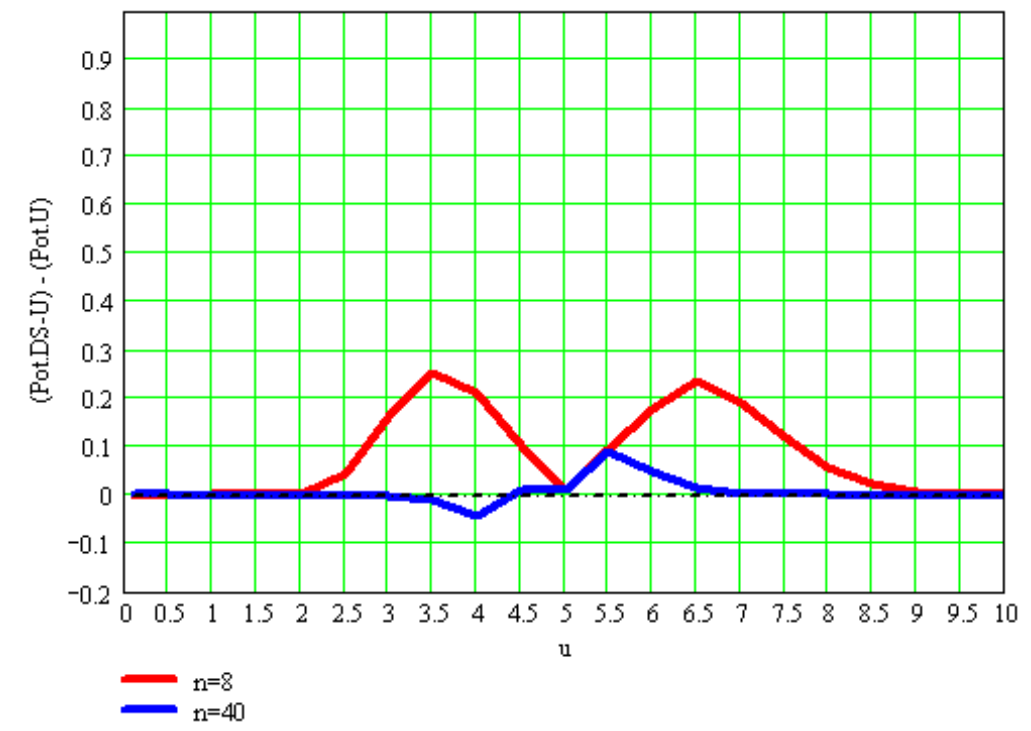

Fig. A.273: $\operatorname{Pot}_{D S-U}-\operatorname{Pot}_{u}$ para $u_{0}=5$ y $\alpha=0.05$ 


\section{Apéndice B}

Comparación de los gráficos de control u y $D S-U$ en

$u_{0} \in\left[u_{\text {inferior }}, u_{\text {superior }}\right]$ 
APÉNDICE B. COMPARACIÓN DE LOS GRÁFICOS DE CONTROL U $Y$ DS-U EN $U_{0} \in\left[U_{I N F E R I O R}, U_{S U P E R I O R}\right]$

\begin{tabular}{|c||c|c|c|}
\hline$u$ & Potencia $_{D S-U}$ & Potencia $_{u}$ & Potencia $_{D S-U}-$ Potencia $_{u}$ \\
\hline 0.1 & 0.844195 & 0.449329 & 0.394866 \\
0.2 & 0.591833 & 0.201897 & 0.389936 \\
0.3 & 0.379615 & 0.090718 & 0.288897 \\
0.4 & 0.231079 & 0.040762 & 0.190316 \\
0.5 & 0.135892 & 0.018321 & 0.117572 \\
0.6 & 0.078011 & $8.272930 \cdot 10^{-3}$ & 0.069738 \\
0.7 & 0.044105 & $3.941482 \cdot 10^{-3}$ & 0.040164 \\
0.8 & 0.024859 & $2.653148 \cdot 10^{-3}$ & 0.022206 \\
0.9 & 0.014206 & $3.895493 \cdot 10^{-3}$ & 0.010310 \\
\hline 1.0 & 0.010276 & $8.566474 \cdot 10^{-3}$ & $1.709825 \cdot 10^{-3}$ \\
\hline 1.1 & 0.014448 & 0.018553 & $-4.104265 \cdot 10^{-3}$ \\
1.2 & 0.026390 & 0.036270 & $-9.880191 \cdot 10^{-3}$ \\
1.3 & 0.045769 & 0.064081 & -0.018312 \\
1.4 & 0.074041 & 0.103676 & -0.029635 \\
1.5 & 0.112428 & 0.155590 & -0.043163 \\
1.6 & 0.161066 & 0.218981 & -0.057915 \\
1.7 & 0.219091 & 0.291712 & -0.072621 \\
1.8 & 0.284791 & 0.370699 & -0.085909 \\
1.9 & 0.355836 & 0.452389 & -0.096552 \\
2.0 & 0.429576 & 0.533255 & -0.103679 \\
\hline
\end{tabular}

Tabla B.1: Valores de potencia obtenidos para $n=8, u_{0}=1 \mathrm{y}$ $\alpha_{t}=0.01$ 
APÉNDICE B. COMPARACIÓN DE LOS GRÁFICOS DE CONTROL U $Y$ DS-U EN $U_{0} \in\left[U_{I N F E R I O R}, U_{S U P E R I O R}\right]$

\begin{tabular}{|c||c|c|c|}
\hline$u$ & Potencia $_{D S-U}$ & Potencia $_{u}$ & Potencia $_{D S-U}-$ Potencia $_{u}$ \\
\hline 0.1 & 0.909796 & 0.952577 & -0.042781 \\
0.2 & 0.735759 & 0.783358 & -0.047599 \\
0.3 & 0.557829 & 0.569709 & -0.011880 \\
0.4 & 0.406052 & 0.379911 & 0.026142 \\
0.5 & 0.287575 & 0.238180 & 0.049395 \\
0.6 & 0.200248 & 0.143012 & 0.057236 \\
0.7 & 0.139024 & 0.084370 & 0.054654 \\
0.8 & 0.097249 & 0.052575 & 0.044674 \\
0.9 & 0.068425 & 0.041375 & 0.027050 \\
\hline 1.0 & 0.057473 & 0.047935 & $9.538170 \cdot 10^{-3}$ \\
\hline 1.1 & 0.070305 & 0.071534 & $-1.229609 \cdot 10^{-3}$ \\
1.2 & 0.097878 & 0.111987 & -0.014109 \\
1.3 & 0.133269 & 0.168405 & -0.035136 \\
1.4 & 0.176733 & 0.238580 & -0.061847 \\
1.5 & 0.228289 & 0.318987 & -0.090697 \\
1.6 & 0.286395 & 0.405243 & -0.118848 \\
1.7 & 0.348959 & 0.492756 & -0.143796 \\
1.8 & 0.413826 & 0.577342 & -0.163516 \\
1.9 & 0.478960 & 0.655677 & -0.176717 \\
2.0 & 0.542570 & 0.725505 & -0.182936 \\
\hline
\end{tabular}

Tabla B.2: Valores de potencia obtenidos para $n=8, u_{0}=1 \mathrm{y}$ $\alpha_{t}=0.05$ 
APÉNDICE B. COMPARACIÓN DE LOS GRÁFICOS DE CONTROL U $Y$ DS-U EN $U_{0} \in\left[U_{I N F E R I O R}, U_{S U P E R I O R}\right]$

\begin{tabular}{|c||c|c|c|}
\hline$u$ & Potencia $_{D S-U}$ & Potencia $_{u}$ & Potencia $_{D S-U}-$ Potencia $_{u}$ \\
\hline 0.1 & 1 & 1 & $8.961720 \cdot 10^{-12}$ \\
0.2 & 0.999999 & 0.999996 & $2.973202 \cdot 10^{-6}$ \\
0.3 & 0.999518 & 0.998527 & $9.908087 \cdot 10^{-4}$ \\
0.4 & 0.982882 & 0.963314 & 0.019567 \\
0.5 & 0.869681 & 0.787493 & 0.082188 \\
0.6 & 0.602569 & 0.472850 & 0.129719 \\
0.7 & 0.304033 & 0.199810 & 0.104224 \\
0.8 & 0.112081 & 0.061012 & 0.051069 \\
0.9 & 0.031412 & 0.014796 & 0.016616 \\
\hline 1.0 & 0.010958 & $9.131637 \cdot 10^{-3}$ & $1.826168 \cdot 10^{-3}$ \\
\hline 1.1 & 0.037697 & 0.034119 & $3.578456 \cdot 10^{-3}$ \\
1.2 & 0.143976 & 0.111937 & 0.032038 \\
1.3 & 0.331318 & 0.261682 & 0.069636 \\
1.4 & 0.548553 & 0.464541 & 0.084012 \\
1.5 & 0.739093 & 0.668064 & 0.071029 \\
1.6 & 0.871484 & 0.825224 & 0.046259 \\
1.7 & 0.945853 & 0.921623 & 0.024229 \\
1.8 & 0.980322 & 0.969841 & 0.010481 \\
1.9 & 0.993769 & 0.989946 & $3.822724 \cdot 10^{-3}$ \\
2.0 & 0.998263 & 0.997067 & $1.196136 \cdot 10^{-3}$ \\
\hline
\end{tabular}

Tabla B.3: Valores de potencia obtenidos para $n=40, u_{0}=1 \mathrm{y}$ $\alpha_{t}=0.01$ 
APÉNDICE B. COMPARACIÓN DE LOS GRÁFICOS DE CONTROL U $Y$ DS-U EN $U_{0} \in\left[U_{I N F E R I O R}, U_{S U P E R I O R}\right]$

\begin{tabular}{|c||c|c|c|}
\hline$u$ & Potencia $_{D S-U}$ & Potencia $_{u}$ & Potencia $_{D S-U}-$ Potencia $_{u}$ \\
\hline 0.1 & 1 & 1 & $2.442491 \cdot 10^{-15}$ \\
0.2 & 1 & 1 & $1.182933 \cdot 10^{-8}$ \\
0.3 & 0.999964 & 0.999944 & $1.934144 \cdot 10^{-5}$ \\
0.4 & 0.997065 & 0.995895 & $1.169785 \cdot 10^{-3}$ \\
0.5 & 0.959141 & 0.947519 & 0.011622 \\
0.6 & 0.804590 & 0.767742 & 0.036847 \\
0.7 & 0.528010 & 0.474881 & 0.053129 \\
0.8 & 0.259678 & 0.216646 & 0.043033 \\
0.9 & 0.100531 & 0.078269 & 0.022262 \\
\hline 1.0 & 0.054277 & 0.047396 & $6.881107 \cdot 10^{-3}$ \\
\hline 1.1 & 0.103656 & 0.106533 & $-2.877319 \cdot 10^{-3}$ \\
1.2 & 0.243173 & 0.254211 & -0.011037 \\
1.3 & 0.446320 & 0.463311 & -0.016991 \\
1.4 & 0.655733 & 0.673712 & -0.017978 \\
1.5 & 0.819154 & 0.833356 & -0.014201 \\
1.6 & 0.919461 & 0.928208 & $-8.746754 \cdot 10^{-3}$ \\
1.7 & 0.969333 & 0.973672 & $-4.338546 \cdot 10^{-3}$ \\
1.8 & 0.989910 & 0.991689 & $-1.778788 \cdot 10^{-3}$ \\
1.9 & 0.997100 & 0.997716 & $-6.158964 \cdot 10^{-4}$ \\
2.0 & 0.999264 & 0.999447 & $-1.833573 \cdot 10^{-4}$ \\
\hline
\end{tabular}

Tabla B.4: Valores de potencia obtenidos para $n=40, u_{0}=1 \mathrm{y}$ $\alpha_{t}=0.05$ 
APÉNDICE B. COMPARACIÓN DE LOS GRÁFICOS DE CONTROL U $Y$ DS-U EN $U_{0} \in\left[U_{I N F E R I O R}, U_{S U P E R I O R}\right]$

\begin{tabular}{|c||c|c|c|}
\hline$u$ & Potencia $_{D S-U}$ & Potencia $_{u}$ & Potencia $_{D S-U}-$ Potencia $_{u}$ \\
\hline 0.1 & 1 & 1 & $3.404805 \cdot 10^{-10}$ \\
0.2 & 0.999998 & 0.999967 & $3.095655 \cdot 10^{-5}$ \\
0.3 & 0.998939 & 0.993935 & $5.004302 \cdot 10^{-3}$ \\
0.4 & 0.971206 & 0.910773 & 0.060432 \\
0.5 & 0.819634 & 0.643698 & 0.175936 \\
0.6 & 0.522034 & 0.313928 & 0.208106 \\
0.7 & 0.238121 & 0.105986 & 0.132135 \\
0.8 & 0.079183 & 0.026008 & 0.053175 \\
0.9 & 0.019998 & $5.150110 \cdot 10^{-3}$ & 0.014847 \\
\hline 1.0 & $4.357539 \cdot 10^{-3}$ & $3.631613 \cdot 10^{-3}$ & $7.259265 \cdot 10^{-4}$ \\
\hline 1.1 & $9.942904 \cdot 10^{-3}$ & 0.017787 & $-7.844464 \cdot 10^{-3}$ \\
1.2 & 0.074202 & 0.068373 & $5.829255 \cdot 10^{-3}$ \\
1.3 & 0.248982 & 0.182409 & 0.066573 \\
1.4 & 0.482848 & 0.361748 & 0.121100 \\
1.5 & 0.691898 & 0.568601 & 0.123297 \\
1.6 & 0.841376 & 0.750648 & 0.090728 \\
1.7 & 0.929826 & 0.876936 & 0.052890 \\
1.8 & 0.973219 & 0.947886 & 0.025333 \\
1.9 & 0.991104 & 0.980911 & 0.010193 \\
2.0 & 0.997403 & 0.993895 & $3.507096 \cdot 10^{-3}$ \\
\hline
\end{tabular}

Tabla B.5: Valores de potencia obtenidos para $n=40, u_{0}=1 \mathrm{y}$ $\alpha_{t}=0.0027$ 
APÉNDICE B. COMPARACIÓN DE LOS GRÁFICOS DE CONTROL U $Y$ DS-U EN $U_{0} \in\left[U_{I N F E R I O R}, U_{S U P E R I O R}\right]$

\begin{tabular}{|c||c|c|c|}
\hline$u$ & Potencia $_{D S-U}$ & Potencia $_{u}$ & Potencia $_{D S-U}-$ Potencia $_{u}$ \\
\hline 0.1 & 1 & 1 & 0 \\
0.5 & 1 & 1 & $-1.962186 \cdot 10^{-11}$ \\
1.0 & 0.999995 & 0.999996 & $-8.008246 \cdot 10^{-7}$ \\
1.5 & 0.998714 & 0.998527 & $1.865991 \cdot 10^{-4}$ \\
2.0 & 0.971156 & 0.963314 & $7.841602 \cdot 10^{-3}$ \\
2.5 & 0.831851 & 0.787493 & 0.044358 \\
3.0 & 0.557687 & 0.472850 & 0.084837 \\
3.5 & 0.279440 & 0.199810 & 0.079631 \\
4.0 & 0.106025 & 0.061012 & 0.045014 \\
4.5 & 0.031536 & 0.014796 & 0.016741 \\
\hline 5.0 & 0.010834 & $9.131637 \cdot 10^{-3}$ & $1.702324 \cdot 10^{-3}$ \\
\hline 5.5 & 0.049532 & 0.034119 & 0.015413 \\
6.0 & 0.204872 & 0.111937 & 0.092935 \\
6.5 & 0.424303 & 0.261682 & 0.162621 \\
7.0 & 0.631107 & 0.464541 & 0.166566 \\
7.5 & 0.794200 & 0.668064 & 0.126136 \\
8.0 & 0.900635 & 0.825224 & 0.075410 \\
8.5 & 0.958233 & 0.921623 & 0.036609 \\
9.0 & 0.984578 & 0.969841 & 0.014737 \\
9.5 & 0.994950 & 0.989946 & $5.003949 \cdot 10^{-3}$ \\
10.0 & 0.998520 & 0.997067 & $1.452921 \cdot 10^{-3}$ \\
\hline
\end{tabular}

Tabla B.6: Valores de potencia obtenidos para $n=8, u_{0}=5 \mathrm{y}$ $\alpha_{t}=0.01$ 
APÉNDICE B. COMPARACIÓN DE LOS GRÁFICOS DE CONTROL U $Y$ DS-U EN $U_{0} \in\left[U_{I N F E R I O R}, U_{S U P E R I O R}\right]$

\begin{tabular}{|c||c|c|c|}
\hline$u$ & Potencia $_{D S-U}$ & Potencia $_{u}$ & Potencia $_{D S-U}-$ Potencia $_{u}$ \\
\hline 0.1 & 1 & 1 & 0 \\
0.5 & 1 & 1 & $-6.883383 \cdot 10^{-15}$ \\
1.0 & 1 & 1 & $7.194004 \cdot 10^{-10}$ \\
1.5 & 0.999961 & 0.999944 & $1.662137 \cdot 10^{-5}$ \\
2.0 & 0.997392 & 0.995895 & $1.497476 \cdot 10^{-3}$ \\
2.5 & 0.966106 & 0.947519 & 0.018586 \\
3.0 & 0.837703 & 0.767742 & 0.069961 \\
3.5 & 0.592672 & 0.474881 & 0.117791 \\
4.0 & 0.327621 & 0.216646 & 0.110975 \\
4.5 & 0.141988 & 0.078269 & 0.063719 \\
\hline 5.0 & 0.056858 & 0.047396 & $9.462188 \cdot 10^{-3}$ \\
\hline 5.5 & 0.088454 & 0.106533 & -0.018079 \\
6.0 & 0.258284 & 0.254211 & $4.073142 \cdot 10^{-3}$ \\
6.5 & 0.483488 & 0.463311 & 0.020177 \\
7.0 & 0.684567 & 0.673712 & 0.010856 \\
7.5 & 0.832783 & 0.833356 & $-5.732258 \cdot 10^{-4}$ \\
8.0 & 0.923314 & 0.928208 & $-4.894139 \cdot 10^{-3}$ \\
8.5 & 0.969368 & 0.973672 & $-4.303851 \cdot 10^{-3}$ \\
9.0 & 0.989240 & 0.991689 & $-2.449217 \cdot 10^{-3}$ \\
9.5 & 0.996643 & 0.997716 & $-1.072510 \cdot 10^{-3}$ \\
10.0 & 0.999061 & 0.999447 & $-3.860949 \cdot 10^{-4}$ \\
\hline
\end{tabular}

Tabla B.7: Valores de potencia obtenidos para $n=8, u_{0}=5 \mathrm{y}$ $\alpha_{t}=0.05$ 
APÉNDICE B. COMPARACIÓN DE LOS GRÁFICOS DE CONTROL U $Y$ DS-U EN $U_{0} \in\left[U_{I N F E R I O R}, U_{S U P E R I O R}\right]$

\begin{tabular}{|c||c|c|c|}
\hline$u$ & Potencia $_{D S-U}$ & Potencia $_{u}$ & Potencia $_{D S-U}-$ Potencia $_{u}$ \\
\hline 0.1 & 1 & 1 & 0 \\
0.5 & 1 & 1 & $-2.130145 \cdot 10^{-8}$ \\
1.0 & 0.999825 & 0.999967 & $-1.414701 \cdot 10^{-4}$ \\
1.5 & 0.988894 & 0.993935 & $-5.040762 \cdot 10^{-3}$ \\
2.0 & 0.898709 & 0.910773 & -0.012064 \\
2.5 & 0.664124 & 0.643698 & 0.020426 \\
3.0 & 0.375071 & 0.313928 & 0.061143 \\
3.5 & 0.163085 & 0.105986 & 0.057098 \\
4.0 & 0.056492 & 0.026008 & 0.030484 \\
4.5 & 0.016089 & $5.150110 \cdot 10^{-3}$ & 0.010939 \\
\hline 5.0 & $4.355987 \cdot 10^{-3}$ & $3.631613 \cdot 10^{-3}$ & $7.243740 \cdot 10^{-4}$ \\
\hline 5.5 & 0.014016 & 0.017787 & $-3.771695 \cdot 10^{-3}$ \\
6.0 & 0.114031 & 0.068373 & 0.045658 \\
6.5 & 0.382481 & 0.182409 & 0.200072 \\
7.0 & 0.679531 & 0.361748 & 0.317782 \\
7.5 & 0.856995 & 0.568601 & 0.288394 \\
8.0 & 0.938416 & 0.750648 & 0.187768 \\
8.5 & 0.974943 & 0.876936 & 0.098007 \\
9.0 & 0.990693 & 0.947886 & 0.042807 \\
9.5 & 0.996858 & 0.980911 & 0.015947 \\
10.0 & 0.999030 & 0.993895 & $5.134396 \cdot 10^{-3}$ \\
\hline
\end{tabular}

Tabla B.8: Valores de potencia obtenidos para $n=8, u_{0}=5 \mathrm{y}$ $\alpha_{t}=0.0027$ 


\section{Apéndice $\mathrm{C}$}

\section{Código fuente para la optimización de la curva de potencia del gráfico $u$ en un valor} $u_{1}$

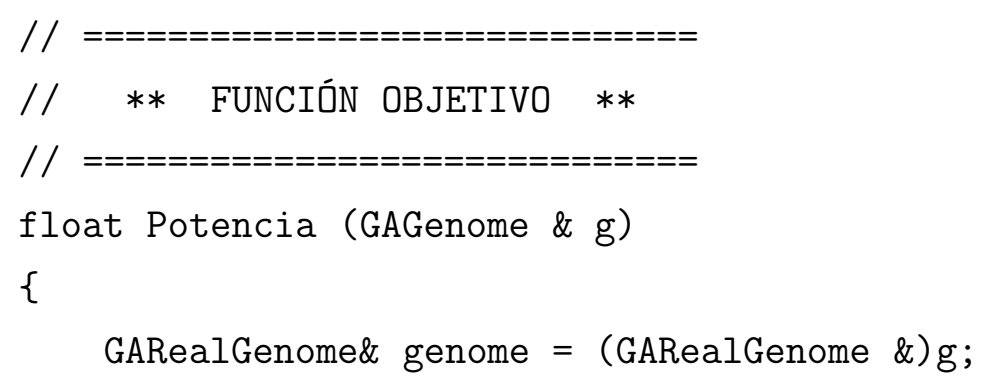


APÉNDICE C. CÓDIGO FUENTE PARA LA OPTIMIZACIÓN DE LA CURVA DE POTENCIA DEL GRÁFICO U EN UN VALOR U.

double $\mathrm{C}=0.0, \mathrm{C} 0=0.0, \mathrm{C} 1=0.0, \mathrm{C} 2=0.0, \mathrm{C} 3=0.0$;

float pLCI, pLAI, pLCI2, pLAS, pLCS, pLCS2, LCI , LAI , LCI2, LAS, LCS , LCS2;

int $n 1, n 2, i, j, k, 1$, contador;

int $\operatorname{dec}=1$;

int $\operatorname{sign}=1$;

int ndig = 1 ;

double $\mathrm{Clas} 0=0.0, \mathrm{Clas}=0.0$;

int $d, f$;

// ** ASIGNACIÓN DE LOS GENOMAS **//

LCI $=$ genome.gene $(0)$;

LAI $=$ genome $\cdot$ gene $(1) ;$

LCI2 $=$ genome $\cdot$ gene $(2)$;

LAS $=$ genome $\cdot$ gene $(3)$;

LCS $=$ genome.gene $(4)$;

LCS2 $=$ genome $\cdot$ gene $(5)$;

$\mathrm{n} 1$ = genome.gene (6);

$\mathrm{n} 2=$ genome.gene $(7)$;

// Verificar que los límites son lógicos //

if $((\mathrm{LCS}>\mathrm{LAS}) \& \&(\mathrm{LAS}>\mathrm{LAI}) \& \&(\mathrm{LAI}>\mathrm{LCI}) \& \&(\mathrm{LCS} 2>\mathrm{LAS}) \& \&(\mathrm{LCI} 2>\mathrm{LCI}))$

\{

/// ** DEFINICIÓN ALFA1 DEL GRÁFICO NUEVO DS-U** ///

// Alfa Superior//

/// PARTE A ///

$\mathrm{A}=0.0 ; / /$ Inicializo A

$i=0$;

$j=f \operatorname{loor}$ (LCS) ;

$\mathrm{A} 0=0.0$; 
APÉNDICE C. CÓDIGO FUENTE PARA LA OPTIMIZACIÓN DE LA CURVA DE POTENCIA DEL GRÁFICO U EN UN VALOR U.

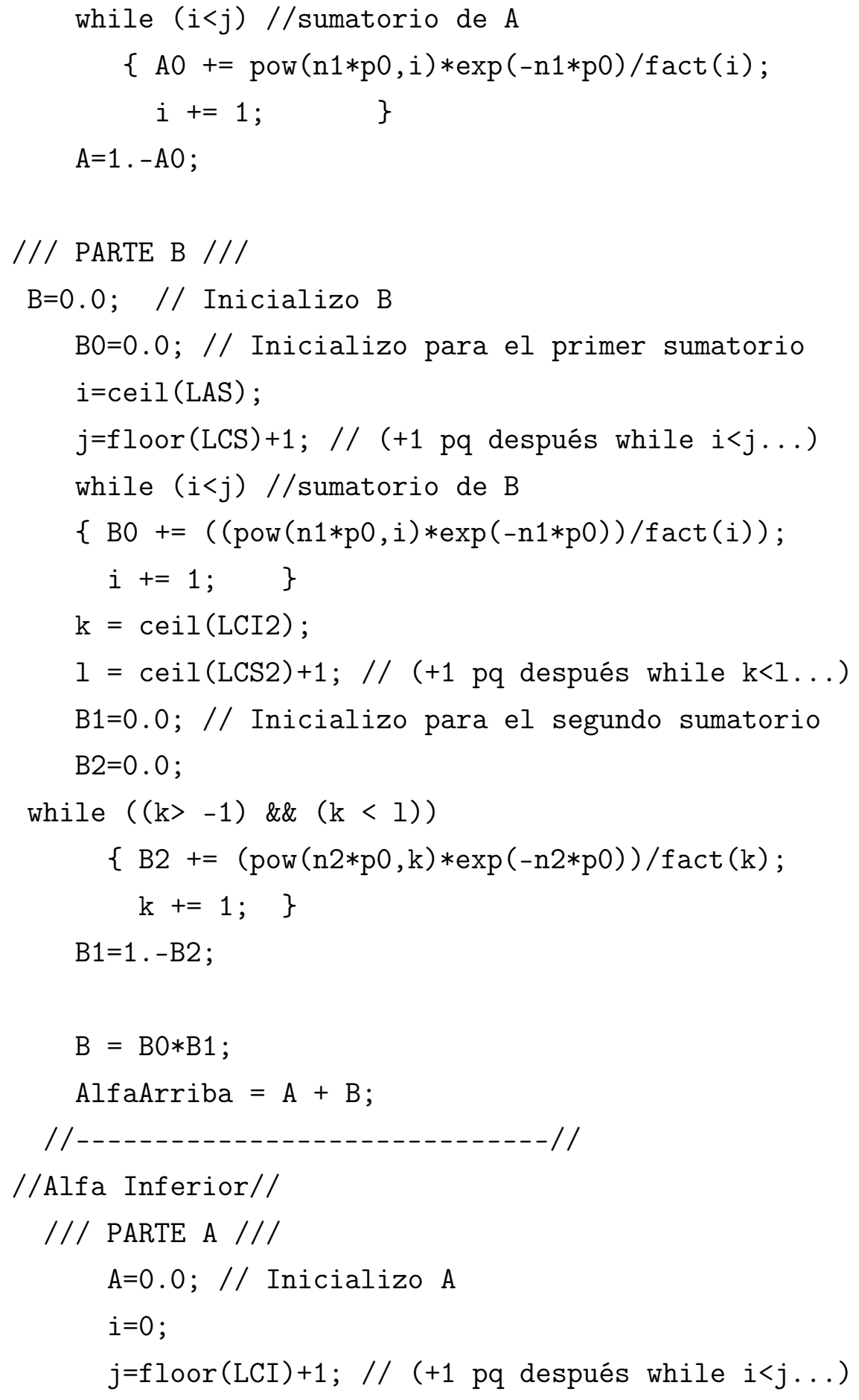


APÉNDICE C. CÓDIGO FUENTE PARA LA OPTIMIZACIÓN DE LA CURVA DE POTENCIA DEL GRÁFICO U EN UN VALOR U.

$\{\mathrm{A} 0+=((\operatorname{pow}(\mathrm{n} 1 * \mathrm{p} 0, \mathrm{i}) * \exp (-\mathrm{n} 1 * \mathrm{p} 0)) / \mathrm{fact}(\mathrm{i})) ; \quad\}$

$\mathrm{A}=\mathrm{AO}$;

// PARTE B //

$\mathrm{B}=0.0 ; \quad \mathrm{BO}=0.0 ; \quad / /$ Inicializo B y BO

$i=\operatorname{ceil}(L C I) ; \quad j=f l o o r(L A I)+1$;

for $(i ; i<j ; i++) / /$ sumatorio de $B$

$\{\mathrm{B} 0+=((\operatorname{pow}(\mathrm{n} 1 * \mathrm{p} 0, \mathrm{i}) * \exp (-\mathrm{n} 1 * \mathrm{p} 0)) / \mathrm{fact}(\mathrm{i})) ; \quad\}$

$\mathrm{B}=\mathrm{BO}$;

// Tamaño redondeado al entero superior

Tamany $=\operatorname{ceil}(\mathrm{n} 1+(\mathrm{n} 2 *(\mathrm{~A}+\mathrm{B})))$;

$/ / / * *$ FINAL TAMAÑO MEDIO GRÁFICO DS-U **///

// Comprobamos si alfa clásico y alfa DS-U son similares //

// para maximizar la diferencia en otro valor de p1 //

if $((a l f a 1>0.06)$ \&\& $($ alfa1<0.07) $) / /$

if $(($ Tamany $>(0.8 * \operatorname{cn} 1)) \& \&($ Tamany $<(1.2 * \operatorname{cn} 1)))$

\{

$/ / / * *$ DEFINICIÓN POTENCIA GRÁFICO DS-U ** ///

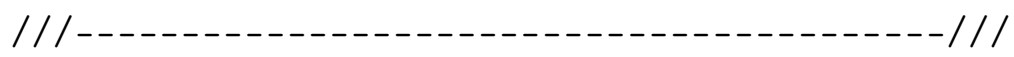

/// PARTE A ///

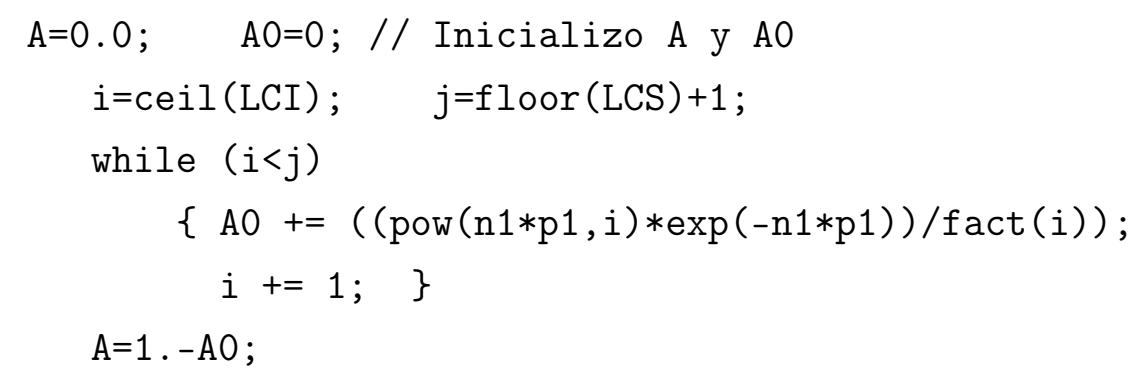

/// PARTE B /// 
APÉNDICE C. CÓDIGO FUENTE PARA LA OPTIMIZACIÓN DE LA CURVA DE POTENCIA DEL GRÁFICO U EN UN VALOR $U_{1}$.

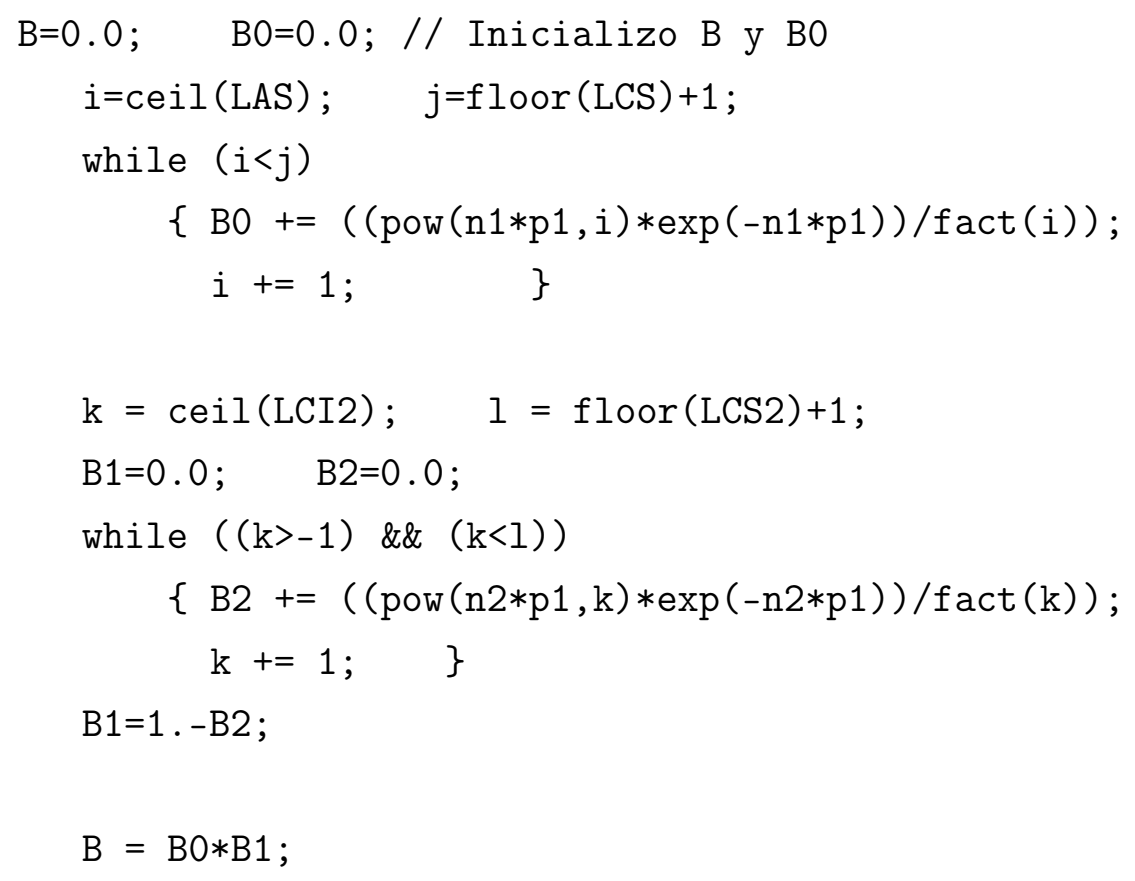


APÉNDICE C. CÓDIGO FUENTE PARA LA OPTIMIZACIÓN DE LA CURVA DE POTENCIA DEL GRÁFICO U EN UN VALOR U.

ValorPotencia $=\mathrm{A}+\mathrm{B}+\mathrm{C}$;

/// ** --- FINAL POTENCIA GRÁFICO DS_U --- ** ///

ValorDiferencia = ValorPotencia - ValorPotenciaClasica;

return ValorDiferencia;

\}

else

\{ return $-1 ;\} / /$ Tamaño muestra distinto a clásico

else

\{ return $-2 ;\} / /$ El valor de los alfa es muy distinto

\}

else

\{

return -5; // Límites no lógicos

\}

\} 


\section{Apéndice D}

\section{Código fuente para Optimización de la curva de potencia del gráfico \\ $\boldsymbol{u}$ en $u_{0} \in\left[u_{i}, u_{s}\right]$}

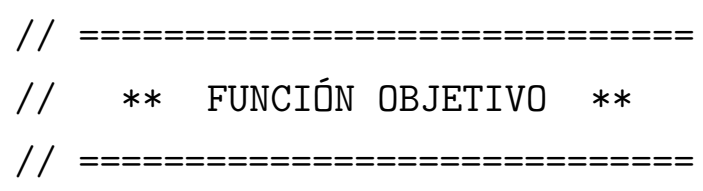

float Potencia (GAGenome \& $g$ ) \{

GARealGenome\& genome $=($ GARealGenome \& $) \mathrm{g}$;

double ValorDiferencia=0.0, AlfaArriba, AlfaAbajo, alfa1C;

double AlfaArribaC, AlfaAbajoC, ValorSuma=0.0;

double $\mathrm{A} 0=0.0, \mathrm{~A}=0.0, \mathrm{~B}=0.0, \mathrm{~B} 0=0.0, \mathrm{~B} 1=0.0, \mathrm{~B} 2=0.0$;

double $\mathrm{C}=0.0, \mathrm{C} 0=0.0, \mathrm{C} 1=0.0, \mathrm{C} 2=0.0, \mathrm{C} 3=0.0$;

float pLCI, pLAI, pLCI2, pLAS, pLCS, pLCS2, LCI , LAI , LCI2, LAS, LCS , LCS2; 
APÉNDICE D. CÓDIGO FUENTE PARA OPTIMIZACIÓN DE LA CURVA DE POTENCIA DEL GRÁFICO U EN $U_{0} \in\left[U_{I}, U_{S}\right]$

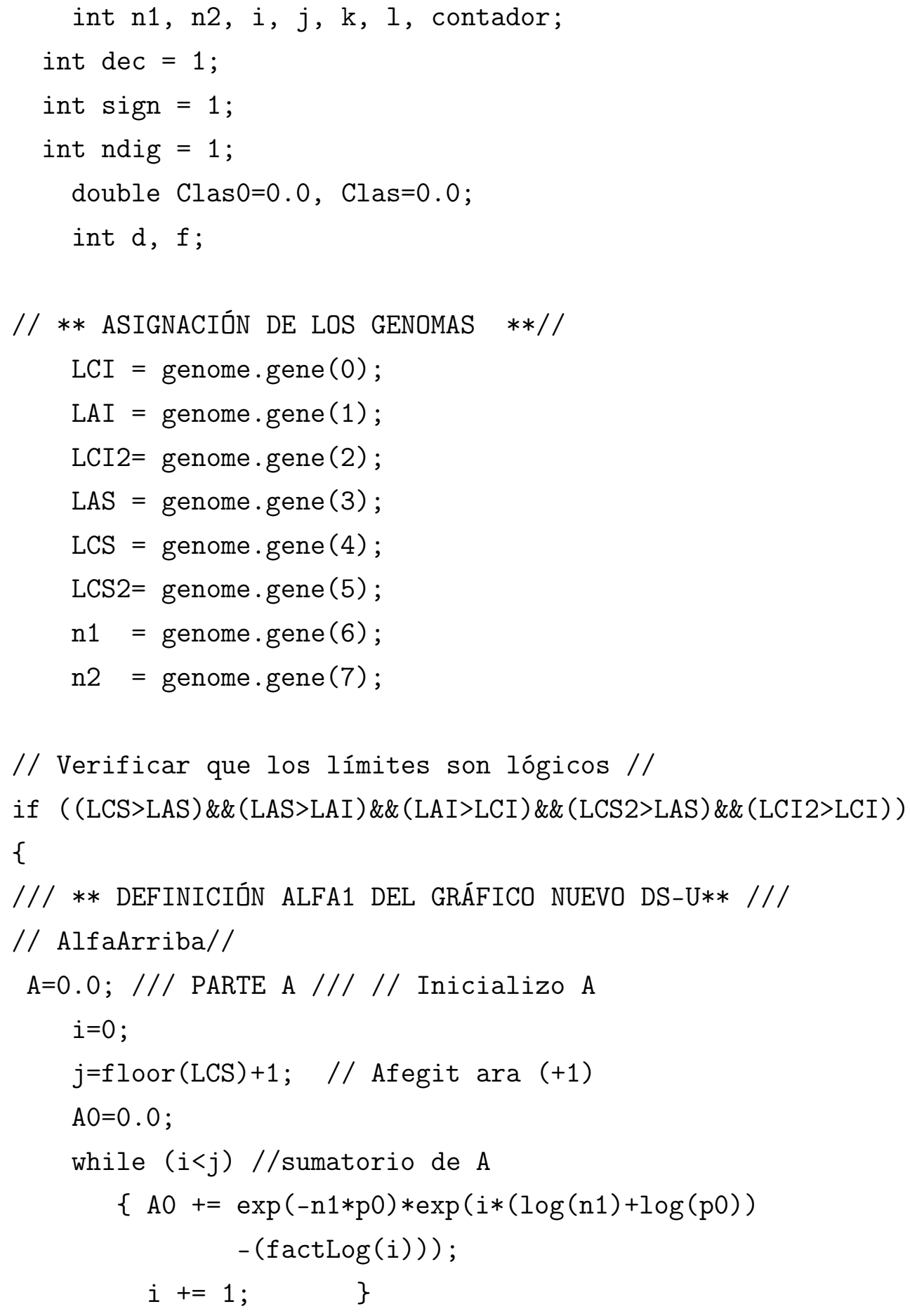


APÉNDICE D. CÓDIGO FUENTE PARA OPTIMIZACIÓN DE LA CURVA DE POTENCIA DEL GRÁFICO U EN $U_{0} \in\left[U_{I}, U_{S}\right]$

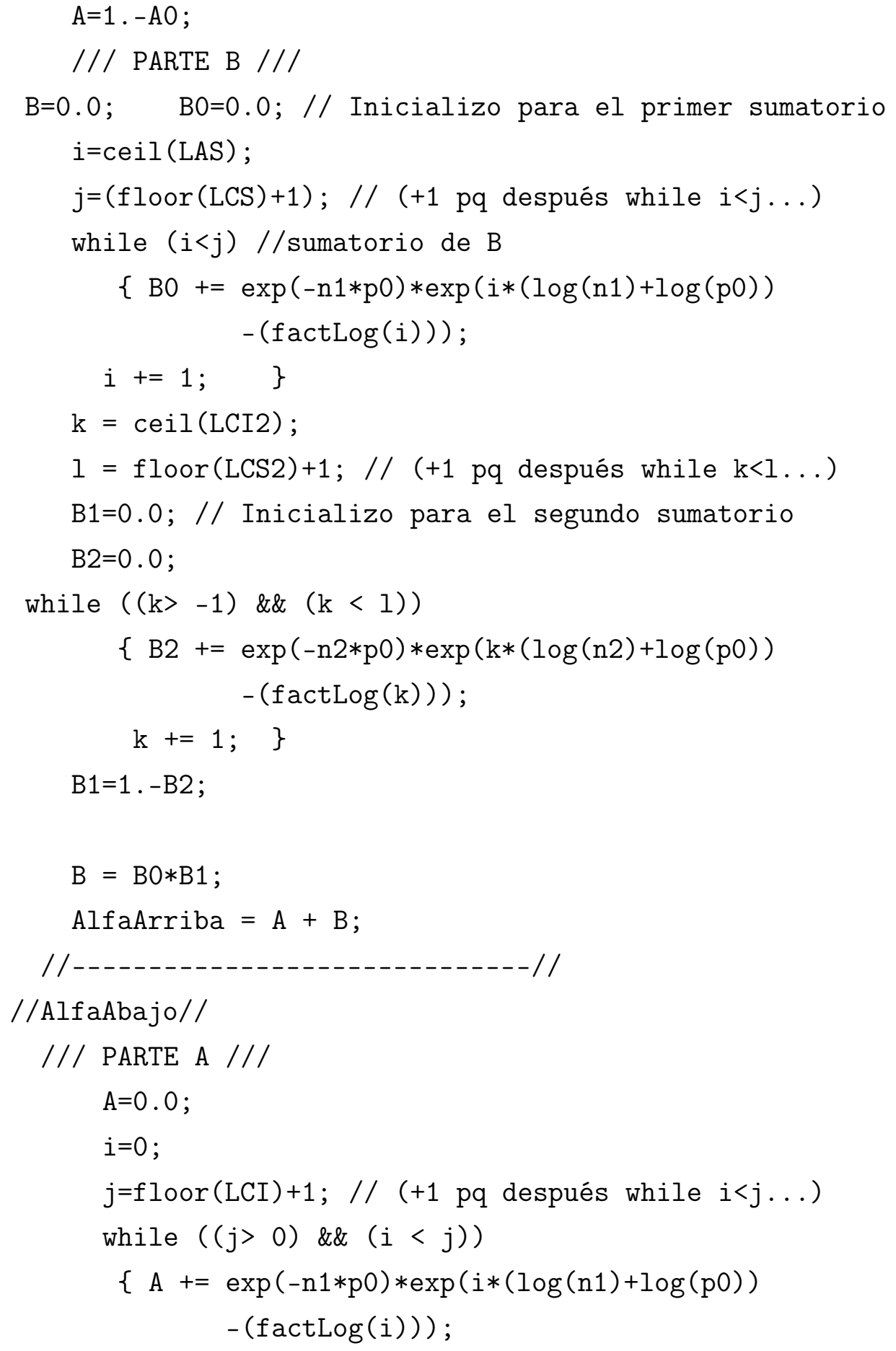


APÉNDICE D. CÓDIGO FUENTE PARA OPTIMIZACIÓN DE LA CURVA DE POTENCIA DEL GRÁFICO U EN $U_{0} \in\left[U_{I}, U_{S}\right]$

$$
\text { i }+=1 ; \quad\}
$$

/// PARTE B ///

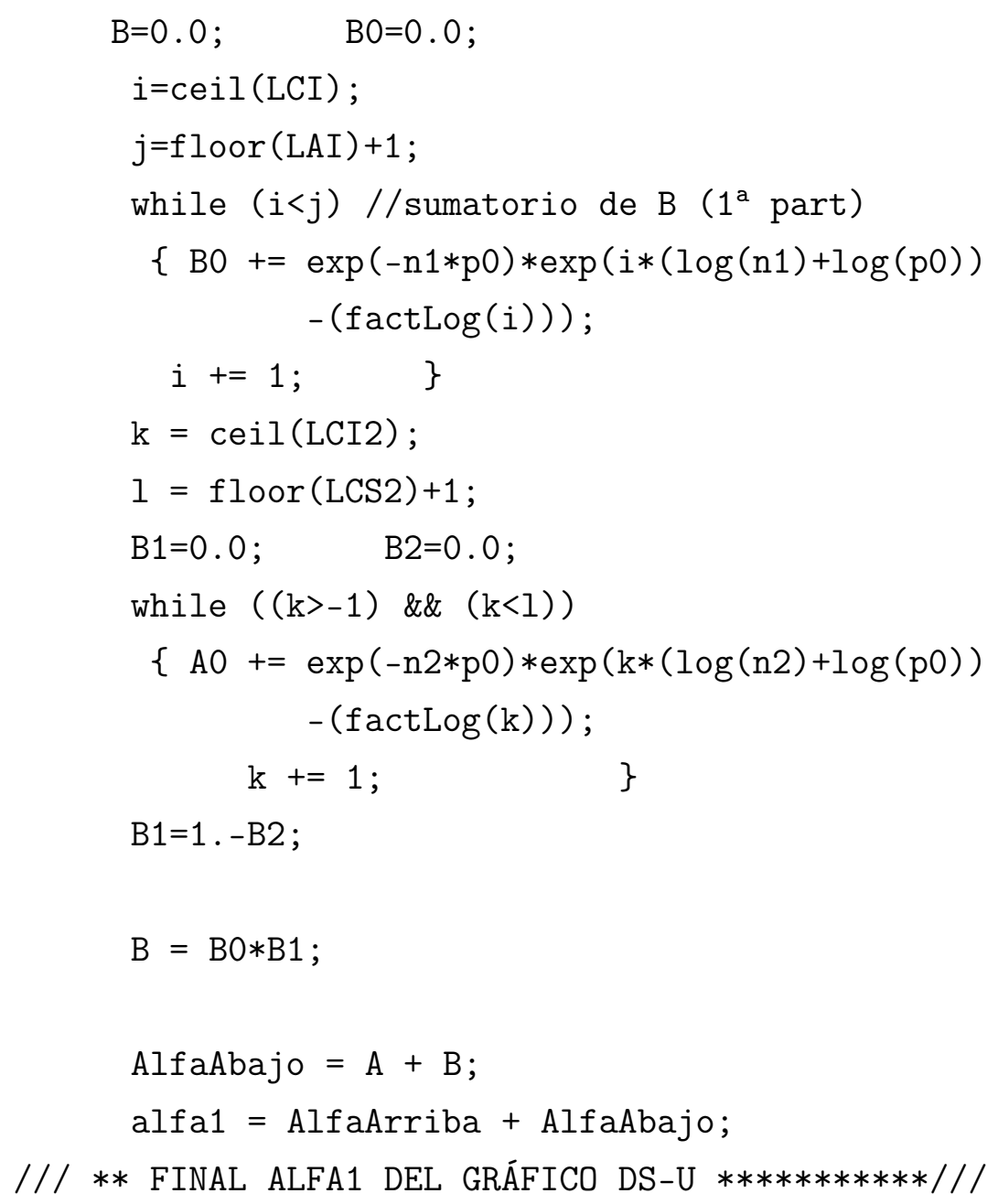


APÉNDICE D. CÓDIGO FUENTE PARA OPTIMIZACIÓN DE LA CURVA DE POTENCIA DEL GRÁFICO U EN $U_{0} \in\left[U_{I}, U_{S}\right]$

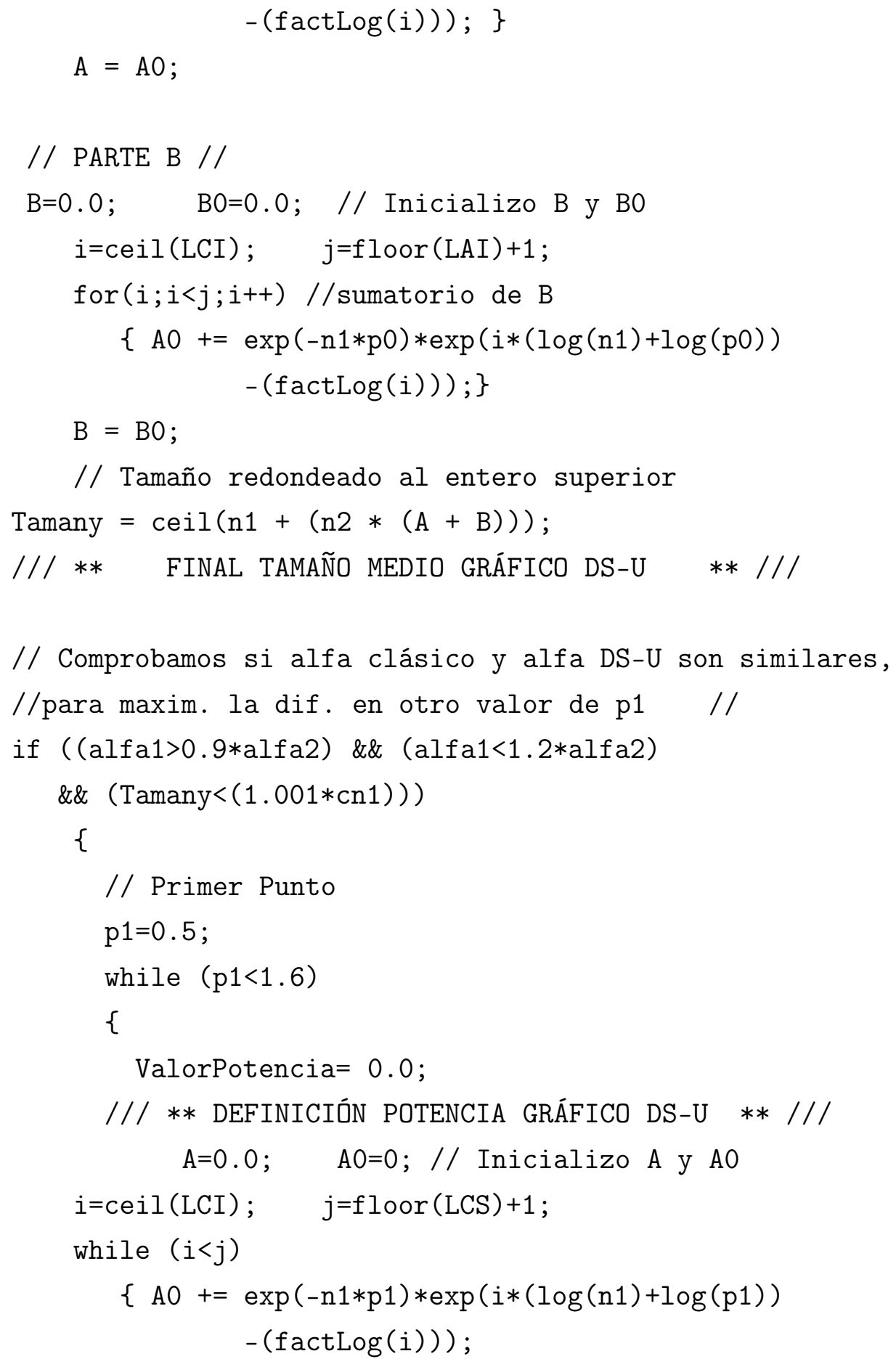


APÉNDICE D. CÓDIGO FUENTE PARA OPTIMIZACIÓN DE LA CURVA DE POTENCIA DEL GRÁFICO U EN $U_{0} \in\left[U_{I}, U_{S}\right]$

$$
\text { i }+=1 ; \quad\}
$$

$A=1 .-A 0$;

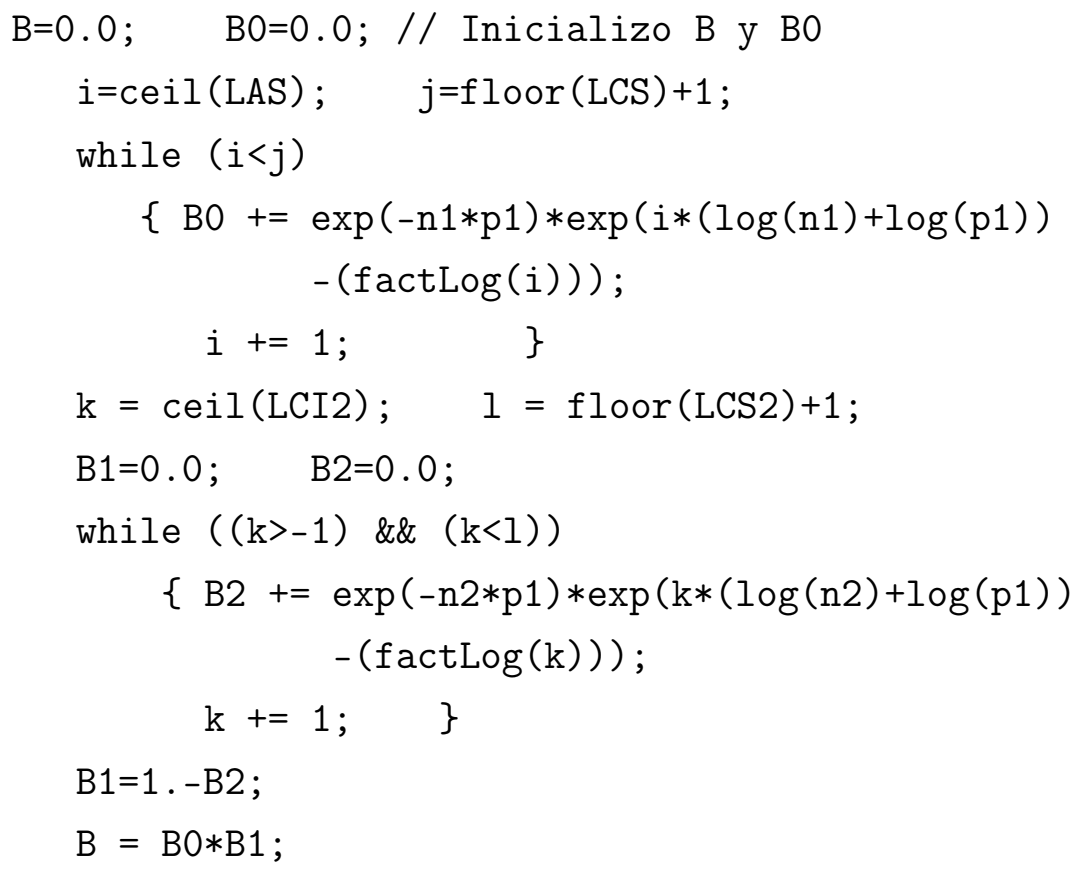


APÉNDICE D. CÓDIGO FUENTE PARA OPTIMIZACIÓN DE LA CURVA DE POTENCIA DEL GRÁFICO U EN $U_{0} \in\left[U_{I}, U_{S}\right]$

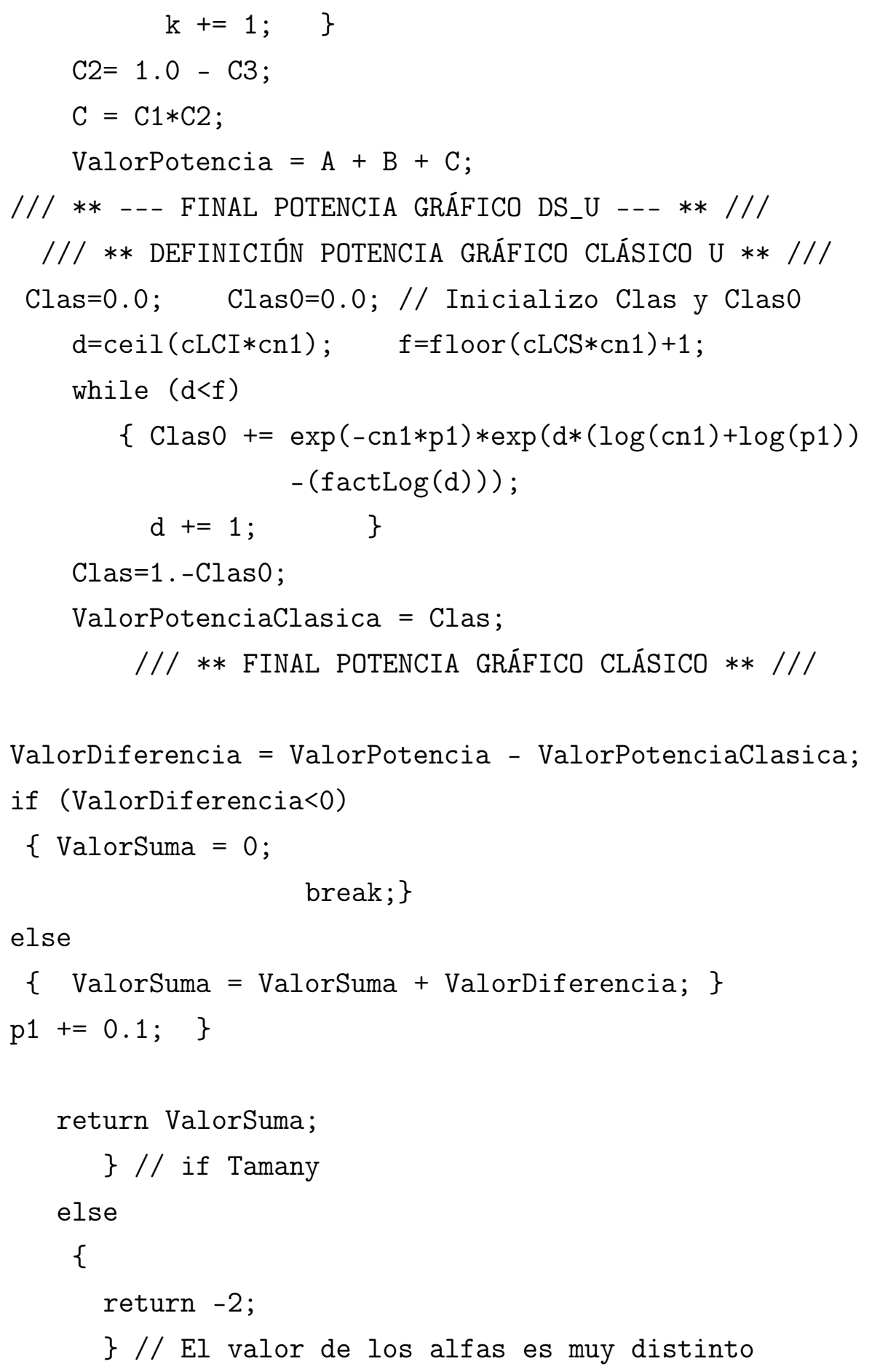


APÉNDICE D. CÓDIGO FUENTE PARA OPTIMIZACIÓN DE LA CURVA DE POTENCIA DEL GRÁFICO U EN $U_{0} \in\left[U_{I}, U_{S}\right]$

\}//if Alfa

return -5; // Límites no lógicos

\} // if final

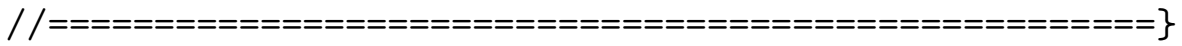

